\title{
Hsa-microRNA- $|8|$ a is a regulator of a number of cancer genes and a biomarker for endometrial carcinoma in patients: a bioinformatic and clinical study and the therapeutic implication
}

\author{
This article was published in the following Dove Press journal: \\ Drug Design, Development and Therapy \\ 18 February 2015 \\ Number of times this article has been viewed
}

\author{
Shuming $\mathrm{He}^{1}$ \\ Shumei Zeng' \\ Zhi-Wei Zhou ${ }^{2,3}$ \\ Zhi-Xu He ${ }^{3}$ \\ Shu-Feng Zhou ${ }^{2,3}$ \\ 'Department of Obstetrics and \\ Gynecology, Xiaolan People's \\ Hospital affiliated to Southern \\ Medical University, Zhongshan, \\ Guangdong, People's Republic of \\ China; ${ }^{2}$ Department of Pharmaceutical \\ Sciences, College of Pharmacy, \\ University of South Florida, Tampa, \\ FL, USA; ${ }^{3}$ Guizhou Provincial Key \\ Laboratory for Regenerative \\ Medicine, Stem Cell and Tissue \\ Engineering Research Center and \\ Sino-US Joint Laboratory for Medical \\ Sciences, Guiyang Medical University, \\ Guiyang, Guizhou, People's Republic \\ of China
}

Correspondence: Shuming He

Department of Obstetrics and Gynecology, Xiaolan People's Hospital affiliated to Southern Medical University, Xiaolan, Zhongshan 528415,

Guangdong, People's Republic of China

Tel +867608862 6236

Fax +8676022836688

Email 13703036288@I39.com

Shu-Feng Zhou

Department of Pharmaceutical Sciences, College of Pharmacy, University of South Florida, 1290I Bruce B. Downs Blvd., MDC 30, Tampa, FL 336।2, USA

$\mathrm{Tel}+\mathrm{I} 8139746276$

Fax + I 8I39059885

Email szhou@health.usf.edu

\begin{abstract}
The aberrant expression of human microRNA-181a-1 (hsa-miR-181a) has been implicated in the pathogenesis of various cancers, serving as an oncogene or a tumor suppressor. However, the role of hsa-miR-181a in the pathogenesis of endometrial carcinoma (EC) and its clinical significance are unclear. This study aimed to search for the molecular targets of hsamiR-181a using bioinformatic tools and then determine the expression levels of hsa-miR-181a in normal, hyperplasia, and EC samples from humans. To predict the targets of hsa-miR-181a, ten different algorithms were used, including miRanda-mirSVR, DIANA microT v5.0, miRDB, RNA22 v2, TargetMiner, TargetScan 6.2, PicTar, MicroCosm Targets v5, and miRWALK. Two algorithms, TarBase 6.0 and miRTarBase, were used to identify the validated targets of hsamiR-181a-5p (a mature product of hsa-miR-181a), and the web-based Database for Annotation, Visualization and Integrated Discovery (DAVID) 6.7 was used to provide biological functional interpretation of the validated targets of hsa-miR-181a-5p. A total of 78 formalin-fixed, paraffinembedded tissue specimens from 65 patients and 13 healthy subjects were collected and examined, including normal endometrium $(n=13)$, endometrial hyperplasia $(n=18)$, and EC (37 type I and 10 type II EC cases). Our bioinformatic studies have showed that hsa-miR-181a might regulate a large number of target genes that are important in the regulation of critical cell processes, such as cell fate, cell survival, metabolism, and cell death. To date, 313 targets of hsa-miR-181a have been validated, and 22 of these targets are cancer genes. The precision of predictions by all the algorithms for hsa-miR-181a-1's targets was low. Many of these genes are involved in tumorigenesis of various cancers, including EC, based on the DAVID and Kyoto Encyclopedia of Genes and Genomes (KEGG) pathway analysis. In comparison with normal endometrial tissue, the expression level of hsa-miR-181a was significantly increased in type I and type II EC $(P<0.05)$, and type II EC exhibited a significant higher expression level of hsa-miR-181a than that in type I EC $(P<0.05)$. In addition, there was a significant increase in the expression level of hsa-miR-181a in type II EC compared with endometrial hyperplasia $(P<0.05)$. Taken together, these results suggest that hsa-miR-181a may serve as an oncogene in endometrial tumorigenesis and that hsa-miR-181a might be used as a new biomarker in the prediction of prognosis of EC in clinical practice. More functional and mechanistic studies are needed to validate the role of hsa-miR-181a in the development, progression, and metastasis of EC.
\end{abstract}

Keywords: RT-PCR, endometrial cancer, development, progression

\section{Introduction}

microRNAs (miRNAs) are a large group of noncoding small RNAs with 20-25 nucleotides that have a capability of regulating gene expression at posttranscriptional levels by antisense complementarily to specific target messenger (m)RNAs. ${ }^{1,2}$ Based 
on miRBase version 21 released in June 2014 (http://www. mirbase.org/), there are 1,881 miRNA precursors and 2,588 mature miRNAs in humans. miRNAs are transcribed as $\sim 70$ nucleotide stem-loop precursors and subsequently processed by the cytoplasmic RNase III-type enzyme Dicer to generate $\sim 22$ nucleotide mature products that can target and modulate protein expression by inhibiting translation and/or inducing degradation of target mRNAs. The mature miRNA is incorporated into a RNA-induced silencing complex (RISC), which recognizes target mRNAs through imperfect base pairing with the miRNA. miRNAs act as adaptors that employ a silencing complex to target mRNAs by selective base-pairing, primarily in the $3^{\prime}$-untranslated region (3'-UTR). Target interaction does not require perfect complementarity between microRNA and mRNA sequences, although nearperfect base-pairing in a small region in the $5^{\prime}$-end (positions 2-8) of the microRNA (sometimes termed "seed") appears to be one of the key determinants of target recognition. miRNAs regulate almost every signaling pathway and play crucial roles in diverse biological processes, such as development, differentiation, apoptosis, and proliferation. ${ }^{1-3}$ It has been shown that aberrant expression of miRNAs is involved in the development and progression of many types of cancer through regulation of functional proteins and the network of signaling pathways related to cell proliferation, cell migration and invasion, programmed cell death, and cell survival. ${ }^{3-7}$ It has been proposed that miRNAs can function as tumor suppressors or oncogenes, targeting other oncogenes and/or tumor-suppressors to modulate cancer development, progression, and metastasis. ${ }^{1,5-8}$

An extremely large number of potential target sites exist for any given miRNA, and the process of validating a potential miRNA target in the laboratory is time consuming and costly. A computational approach to prediction of miRNA targets facilitates the process of narrowing down potential target sites for experimental validation, which is a critical initial step in identifying miRNA-target interactions. Several useful algorithms/tools provide microRNA target predictions based on sequence complementarity to target sites, with emphasis on perfect or near-perfect base-pairing in the seed region and sequence conservation. ${ }^{9,10}$ These tools for miRNA target prediction, encompassing a range of different computational approaches, from the modeling of physical interactions to the incorporation of machine learning, are mostly based on seed match, conservation, free energy, and site accessibility. ${ }^{10}$

Endometrial cancer (EC) is the sixth most common cancer in women worldwide, with at least 320,000 new cases being diagnosed and 74,000 women who die from this disease every year. ${ }^{11}$ In the United States, there was an estimation of 52,630 new cases and 8,590 deaths due to EC in $2014 .{ }^{12}$ In the United Kingdom, there were 8,474 women diagnosed with EC and 1,914 deaths from EC in 2011. ${ }^{11}$ In the People's Republic of China, the incidence of EC is much lower than Western countries. It contributes about $1 \%$ of the world's new EC cases. ${ }^{11}$

There are two types of EC, type I and type II, with different molecular expression profiles and histopathological and clinical behaviors. ${ }^{13,14}$ Type I EC, accounting for $75 \%$ of EC, is estrogen-dependent with endometrioid morphology, and the 5 -year survival rate is $80 \%-85 \%$. $^{15}$ Type II EC exhibits poorly differentiated endometrioid and serous histological alterations with myometrial invasion and extrauterine spread, and the 5-year survival rate is about $35 \%$. Type I EC is related to hyperestrogenism, associated with endometrial hyperplasia, frequent expression of estrogen and progesterone receptors (ER and PR), and younger age, whereas type II EC is unrelated to estrogen and is associated with atrophic endometrium, frequent lack of ER and PR, and older age. The morphologic differences in type I and type II EC are mirrored in their molecular genetic profile, with type I showing defects in DNA-mismatch repair and mutations in phosphatase and tensin homolog (PTEN), phosphatidylinositol-4,5-bisphosphate 3-kinase, catalytic subunit $\alpha$ (PIK3CA), V-Ki-ras2 Kirsten rat sarcoma viral oncogene homolog (KRAS), and $\beta$-catenin/CTNNB1, and type II showing chromosomal instability and aneuploidy, p53 mutations, and human epidermal growth factor receptor 2 (HER2)/neu (erbB-2) amplification. ${ }^{16,17}$ Type I EC is associated with chronic exposure to unopposed estrogen and is often preceded by complex atypical hyperplasia. Current therapies for EC include surgery, chemotherapy, radiation therapy, immunotherapy, and biological therapy. ${ }^{18}$ Most cases of EC are diagnosed at an early stage, which has a 5-year survival rate of over $91 \% .{ }^{11}$ However, the prognosis of EC does not meet the long-term survival expectation due to tumor metastasis, lack of effective treatment, and rarity of valid biomarkers to precisely predict therapeutic outcome. ${ }^{19}$ There are lines of evidence that a number of genetic and epigenetic factors have been implicated in the pathogenesis of EC, including abnormality in oncogenes, tumor suppressors, and miRNAs and related signaling pathways. ${ }^{13}$ Alterations in the expression profiles of oncogenes and tumor suppressor genes are the major contributing factors to the initiation, development, progression, and metastasis of EC. However, the association between such alterations and the clinical phenotypes of EC has not been conclusively established yet, and the underlying mechanism for EC etiology remains elusive.

There is an increased interest in the discovery and identification of miRNAs as novel epigenetic biomarkers 
for early detection and prediction of therapeutic outcomes in cancer therapy. ${ }^{20-24}$ So far, there are a number of human miRNAs that have been identified to have a potential role in the development and progression of EC, including hsamiR-181a, hsa-miR-185, hsa-miR-106a, hsa-miR-210, hsa-miR-423, hsa-miR-107, hsa-miR-let7c, and hsa-miR$221 .^{3,4}$ hsa-miR-181a is one of the many miRNAs conserved among the vertebrates that is preferentially expressed in $\mathrm{B}$ lymphocytes of bone marrow, and its ectopic expression in hematopoietic stem/progenitor cells modulates blood cell development. ${ }^{25}$ hsa-miR-181a has been reported to be a key modulatory factor in the regulation of cell proliferation and differentiation at transcriptional and posttranscriptional levels in gastric cancer, acute myeloid leukemia, and other types of cancer, ${ }^{26,27}$ and hsa-miR-181a overexpression resulted in promotion of cell proliferation and migration but inhibition of apoptosis in colorectal cancer. ${ }^{28}$ RalA, one of the Ral family small G proteins, is directly regulated by hsa-miR181a and plays an important role in the development chronic myelogenous leukemia. ${ }^{29}$ hsa-miR-181a and hsa-miR-181b act as tumor suppressors by inducing cell growth inhibition, apoptosis, and repression of invasion in glioma cells. ${ }^{30}$ So far, there have been only scattered reports about the role of a form of miR-181a in EC. ${ }^{4}$ Panda et $\mathrm{al}^{31}$ showed that the expression level of miR-181a-1 was higher in EC than in normal endometrial tissues. However, there is limited information on the association between the expression profile of miR-181a and the development and progression of EC. In this regard, we conducted a comprehensive bioinformatic study to predict the targets of miR-181a-1 and then validate these targets based on published experimental evidence. Finally, we examined the expression levels of miR-181a-1 in different types of EC and the association with the clinical progression of EC.

\section{Material and methods Gene nomenclature}

The miR-181 family includes four members, namely miR-181a, miR-181b, miR-181c, and miR-181d. They are expressed in at least 70 species and various human cancers and are highly conserved in the seed-region sequence and RNA secondary structure. hsa-miR-181a-1 was retrieved from miRBase 21 (http://www.mirbase. org/). The hsa-miR-181a-1 gene (MIR181A1) has been mapped to 1q32.1. miRBase was established in 2002 as the public and central online repository for all published miRNA sequences and associated annotations, and the latest miRBase release of v21 (released in June 2014) contains 28,645 miRNA loci from 223 species (of which 1,881 precursors and 2,588 mature miRNAs are for humans), processed to produce 35,828 mature miRNAs. As compared with miRBase v20, a total of 4,196 new hairpin sequences and 5,441 novel mature products, mainly for bat, horse, goat, cobra, and salmon, have been added, with 72 dubious and misannotated entries removed from version 21. miRBase provides a user-friendly web interface for miRNA data, allowing the user to search using key words or sequences, trace links to the primary literature referencing the miRNA discoveries, analyze genomic coordinates and context, and mine relationships between miRNA sequences. ${ }^{32-34}$ Clusters of miRNA sequences in the genome are highlighted and can be defined and retrieved with any inter-miRNA distance. The mature forms of hsa-miR-181a include hsa-miR-181a-3p and hsa-miR-181a-5p (Table 1).

\section{Prediction of the targets of hsa-miR-I8Ia using various computational algorithms}

Before starting the bench and clinical work, we conducted a bioinformatic study to predict the target genes regulated by hsa-miR-181a-3p and hsa-miR-181a-5p, using ten different algorithms, including miRanda-mirSVR (http://www. microrna.org/), DIANA microT v5.0 (http://diana.cslab. ece.ntua.gr/microT/), miRDB (http://mirdb.org/miRDB/), RNA22 v2 (https://cm.jefferson.edu/rna22v2.0/), TargetMiner (http://www.isical.ac.in/ bioinfo miu/targetminer20.

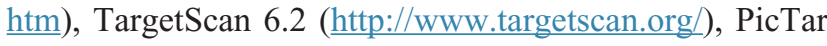
(http://pictar.mdc-berlin.de/), MicroCosm Targets v5 (http:// www.ebi.ac.uk/enright-srv/microcosm/htdocs/targets/v5/), and miRWALK (http://www.umm.uni-heidelberg.de/apps/ zmf/mirwalk/index.html).

miRanda-mirSVR (http://www.microrna.org/) is an online tool that can be used to predict targets and score them. ${ }^{35}$ Unlike most miRNA target predictors, miRanda considers matching along the entire miRNA sequence, and it takes the seed region into account by weighting matches in the seed region more heavily; free energy is calculated by predicting the folding of the miRNA:mRNA hybrid, using the Vienna package. DIANA microT v5.0 currently hosts miRNA target predictions for Homo sapiens, Mus musculus, Drosophila melanogaster, and Caenorhabditis elegans, based on data from Ensembl release 69 and miRBase version $18 .^{36,37}$ miRDB, a web-based database and tool, can predict miRNAs and their targets. ${ }^{38}$ All the targets are predicted by the bioinformatic tool MirTarget2, which has been developed by analyzing thousands of genes impacted by miRNAs, using a support vector machine (SVM) learning machine. RNA22 v2 can be used to predict the targets of miRNAs in 


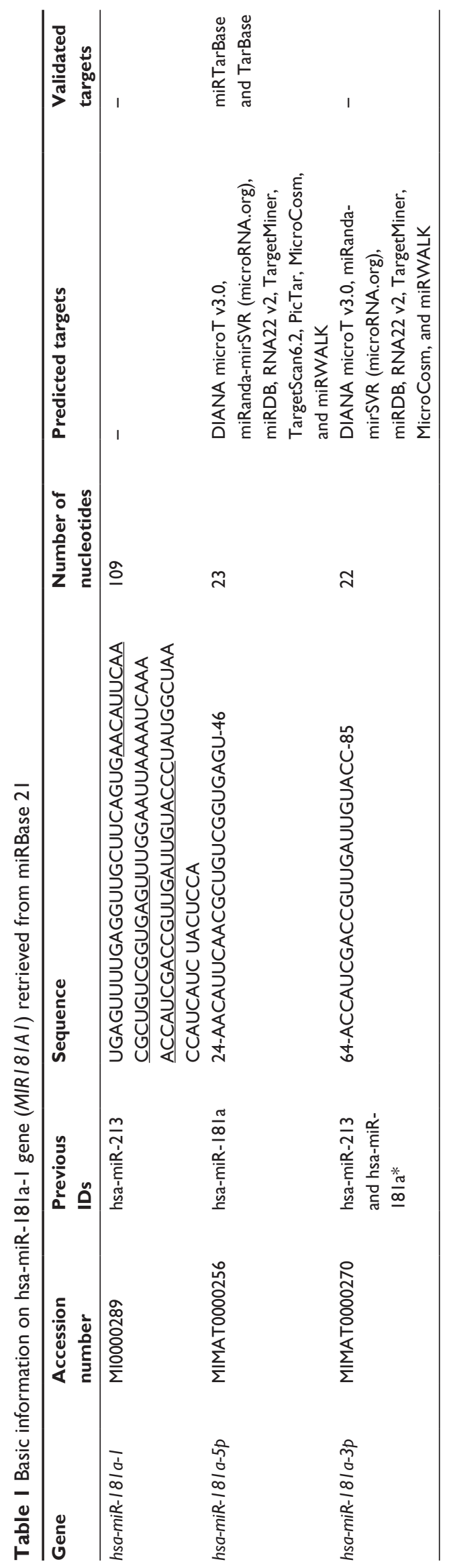

human, mouse, roundworm, and fruit fly. ${ }^{39}$ It allows users to visualize the predictions within a complementary (c)DNA map and also find transcripts where multiple miRNAs of interest target.

TargetMiner is a robust tool for microRNA target prediction with systematic identification of negative examples. ${ }^{40}$ In this algorithm, $\sim 300$ tissue-specific negative examples have been identified, using a novel approach that involves expression profiling of miRNAs and mRNAs, miRNAmRNA structural interactions, and seed-site conservation. TargetScan 6.2 can predict biological targets of miRNAs by searching for the presence of conserved 8-mer and 7-mer sites matching the seed region of each miRNA, ${ }^{41}$ with nonconserved sites being predicted as well. TargetScan is the first computational method used for human miRNA target prediction, that uses mouse, rat, and fish genomes for conservation analysis. In mammals, the prediction is ranked based on the predicted efficacy of targeting as calculated using the context+ scores of the sites. ${ }^{42}$ The context score for a specific site is the sum of the contribution of four features: site-type contribution, 3'-pairing contribution, local nucleobases adenine and uracil contribution, and position contribution. In the current work, the sum of the context scores for each miRNA was calculated, and the most favorable (lowest) was shown. PicTar is an algorithm for the identification of miRNA targets. ${ }^{43}$ In addition, MicroCosm Targets v5 was used to predict the targets that might be regulated by hsamiR-181a. In this tool, there are 851 miRNAs (711 native) with 34,788 targets for humans.

miRWALK is a comprehensive database that provides information on miRNAs from the human, mouse, and rat, on their predicted as well as validated binding sites on their target genes (http://www.umm.uni-heidelberg.de/apps/ zmf/mirwalk/index.html). ${ }^{44}$ miRWALK predicts miRNA binding sites on the complete sequence of all known genes, including all transcripts and mitochondrial genes of the human, mouse, and rat, based on a comparison of identified miRNA binding sites with ten established miRNA-target prediction programs: miRWALK, DIANA-microT v3.0, miRanda, miRDB, PicTar 4 and PicTar 5, PITA (http:// genie.weizmann.ac.il/pubs/mir07/mir07 prediction.html), RNA22, RNAhybrid (http://bibiserv.techfak.uni-bielefeld. de/rnahybrid/submission.html), and TargetScan. In addition, it provides predicted miRNA binding sites on genes associated with 449 human biological pathways and 2,356 Online Mendelian Inheritance in Man (OMIM) disorders. ${ }^{44}$ This algorithm also presents information on experimentally validated miRNA interaction information associated with 
genes, pathways, diseases, organs, OMIM disorders, cell lines, and literature on miRNAs.

\section{Validated targets of hsa-miR-I8 Ia based on TarBase 6.0 and miRTarBase 4.0}

Two algorithms were used to identify the validated targets of miR-181a-5p: TarBase 6.0 and miRTarBase 4.0. TarBase 6.0 is a database that houses a manually curated collection of experimentally supported miRNA targets in 21 species, including human, rat, mouse, virus, Caenorhabditis elegans, Danio rerio (zebrafish), Drosophila, and plant (http://diana. imis.athena-innovation.gr/DianaTools/index.php?r=tarbase/ index)..${ }^{45}$ TarBase 6.0 is the largest available manually curated target database, indexing more than 65,000 miRNA-gene interactions, 16.5 - to 175 -fold more than any other available implementation. The database includes targets derived from specific as well as high-throughput experiments, such as microarrays and proteomics. Specific attention was paid in the inclusion of targets derived from sequencing experiments, such as high-throughput sequencing of RNA isolated by crosslinking immunoprecipitation (HITS-CLIP) and photoactivatable-ribonucleoside-enhanced crosslinking and immunoprecipitation (PAR-CLIP). TarBase 6.0 hosts data derived from three CLIP-Seq and 12 Degradome-Seq studies, significantly more than any other available database. DIANA TarBase 6.0 offers a significant amount of crucial information to the user, including detailed description of the involved genes and miRNAs, a list of publications supporting each interaction, and the experimental methods used for validations, along with their outcomes. The database also provides links to related Kyoto Encyclopedia of Genes and Genomes (KEGG) pathways, as well as to other external databases, such as Ensembl, Uniprot, and RefSeq. It is also equipped with powerful searching and filtering capabilities.

miRTarBase 4.0 has collected 51,460 miRNA-target interactions from 18 species having experimental evidence (http://mirtarbase.mbc.nctu.edu.tw/). ${ }^{46}$ Generally, the collected miRNA-target interactions are validated experimentally by reporter assay, Western blotting, microarray, and next-generation sequencing experiments. There are 4,572 miRNA-target interactions validated by reporter assays or Western blotting experiments.

Cancer genes are defined as "mutated genes that are causally implicated in oncogenesis" based on the criteria by Futreal et $\mathrm{al}^{47}$ (Table S1 gives the full list of cancer genes). The proteins that are encoded by cancer genes normally regulate cell proliferation, cell differentiation, and cell death. Mutations underlying oncogenesis also occur in genes that mediate DNA-repair processes. Currently, more than 1\% of all human genes have been implicated, via mutation, in cancer. Of these, approximately $90 \%$ have been implicated in somatic mutations in cancer, $20 \%$ bear germline mutations that predispose to cancer, and $10 \%$ show both somatic and germline mutations. ${ }^{48}$

\section{Pathway analysis by the Database for Annotation, Visualization and Integrated Discovery (DAVID)}

The web-based DAVID 6.7 (http://david.abcc.ncifcrf. gov $/)^{49,50}$ was used to provide biological functional interpretation of the validated targets of hsa-miR-181a-5p, based on TarBase 6.0 and miRTarBase 4.0. DAVID 6.7 systematically maps a large number of interesting genes in a list to associated Gene Ontology (GO) terms, and then statistically highlights the most overrepresented (enriched) GO terms out of a list of hundreds or thousands of terms..$^{47,48}$ The DAVID Knowledgebase has comprehensively integrated more than 20 types of major gene/protein identifiers and more than 40 well-known functional annotation categories from dozens of public databases, to address the enriched and redundant relationships among many genes to many terms. The protein IDs of the validated targets of hsa-miR-181a-5p from the National Center for Biotechnology Information (NCBI), Protein Information Resource (PIR), and UniProtKB were converted into gene lists, using the Gene ID Conversion Tool in DAVID. By doing so, interesting genes derived from one identifier system can be quickly translated to other gene identifier types preferred by a given annotation resource. The DAVID database adds biological function annotation including GO terms, protein-protein interactions, protein functional domains, disease associations, gene clustering, biopathways, sequence general features, homologies, gene functional summaries, and gene tissue expressions in a network context. ${ }^{47,48}$ The genes of interest were visualized using BioCarta and KEGG pathway maps. The highest classification stringency was selected for functional annotation clustering. Enrichment scores and Fisher's exact test $P$-values (and corresponding false discovery rate [FDR]) were then calculated to identify which functionally related gene groups are significantly enriched in the target list.

\section{Reagents and antibodies}

The RNA inhibitor and Moloney murine leukemia virus reverse transcriptase were obtained from Promega Inc. (Madison, WI, USA). The antigen retrieval solution (ethylenediaminetetraacetic acid [EDTA] method) and Dolichos 
biflorus agglutinin kit were purchased from Maixin Biological Co. Ltd., Fuzhou, Fujian, People's Republic of China. Human monoclonal primary antibodies against (ER, PR), and horseradish peroxidase-conjugated secondary antibodies were bought from Linked-Biotech Pathology Co. Ltd. (Guangzhou, Guangdong, People's Republic of China). MiReasy FFPE Kit and Syber ${ }^{\circledR}$ Green PCR mix were purchased from QIAGEN Inc. (Venlo, the Netherlands).

\section{Sample collection}

Fresh tissue samples were obtained from 65 patients who received uterusectomy and 13 healthy subjects at Xiaolan People's Hospital or Zhongshan People's Hospital, Zhongshan, Guangdong, or at Nanfang Hospital of Southern Medical University, Guangzhou, Guangdong, People's Republic of China, with a mean age of 48 years (range 24-69). We collected the endometrial samples from 47 patients with EC, 18 patients with endometrial hyperplasia, and 13 healthy subjects. There was no preoperative radiotherapy, chemotherapy, or endocrine therapy performed in any of the recruited participants. All samples were fixed with formalin and embedded with paraffin. International Federation of Gynecology and Obstetrics (FIGO) staging was performed according to the FIGO classification. ${ }^{15,51}$ Histological classification of tissue samples was performed according to the World Health Organization (WHO) criteria (www.iarc.fr/ en/.../BB2.pdf), and samples were classified as G1 (well differentiated), G2 (moderately differentiated), or G3 (poorly differentiated). The study design was approved by the Ethics Committees of Xiaolan People's Hospital, Zhongshan People's Hospital, and Nanfang Hospital. Written informed consent was obtained from each participant.

\section{Immunohistochemistry}

Dewaxed and dehydrated sections were first washed with phosphate-buffered saline (PBS) and then incubated with 3\% peroxyl in methanol for 15 minutes to terminate the activity of endogenous peroxidases. The sections were washed with PBS, and antigen retrieval was performed. The sections were immersed into boiled citrate-buffered solution for 10 minutes and blocked with 5\% bovine serum albumin in PBS for 20 minutes at room temperature. Thereafter, the sections were probed with primary antibody against ER or PR overnight in a humidified chamber at $4^{\circ} \mathrm{C}$. On the following day, sections were incubated with biotinylated anti-Rabbit antibody (Boster Biotechnology Ltd., Wuhan, Hubei, People's Republic of China) for 30 minutes at room temperature and then coupled with diaminobenzidine to visualize the expression of the targeted proteins. After all sections were counterstained with hematoxylin, they were dehydrated in ascending ethanol and then mounted using neutral resins. Samples with both ER-positive and PR-positive were classified as type I EC, while samples with both ER-negative and PR-negative were classified as type II EC.,25

\section{Primer design}

The sequences of target gene were retrieved from GenBank (http://www.ncbi.nlm.nih.gov/genbank/) and miRBase (http://www.mirbase.org/). The primers were designed using Primer Designer 2.0, and the sequences are shown in Table 2. All primers were synthesized by Beijing Liu He Synthetic Genomics Ltd., Beijing, People's Republic of China. U6 was used as the internal control.

\section{Total RNA extraction}

A series of sections of thickness $10 \mu \mathrm{m}$ was obtained, and the paraffin was dissolved by xylene treatment. Ten slides were prepared for each sample. Sections with cell content more than $50 \%$ of the area were selected for total RNA extraction. Briefly, sections were washed twice with ethanol in a $1.5 \mathrm{~mL}$ centrifuge tube to remove residual xylene. Total RNA was extracted, and the purity and integrity of the total RNA were examined using a miRNeasy FFPE kit according to the manufacturer's instruction. The purity of the total RNA was tested using an ultraviolet (UV) spectrophotometer. The ratio of A260/A280 between 1.8 and 2.1 was considered as high purity. Electrophoresis was performed to detect the RNA integrity in $1.0 \%$ agarose denaturing gel.

\section{Quantitative real-time polymerase chain reaction ( $q R T-P C R)$ analysis}

RNA was reversely transcribed into cDNA, using a RevertAid First Strand cDNA Synthesis Kit (Thermo Fisher Scientific Inc., Waltham, MA, USA) according to the manufacturer's instruction. The resultant cDNA was subject to qRT-PCR analysis using a Bio-Rad Real-time PCR System (Bio-Rad Laboratories

Table 2 Sequences of the primers for the determination of hsa-miR-I8Ia and U6

\begin{tabular}{lll}
\hline Gene & Forward primer & Reverse primer \\
\hline hsa-miR-I8Ia & GTCGTATCCAGTGCGTGTCGTGGAGTCG & GCAATTGCACTGGATACGACACTCAC \\
U6 & GTCGTATCCAGTGCGTGTCGTGGAGTCGG & CAATTGCACTGGATACGACAAAATATG \\
\hline
\end{tabular}


Inc., Hercules, CA, USA) and Syber green PCR mix. The conditions for RT-PCR were $95^{\circ} \mathrm{C}$ for 6 minutes and then 50 cycles of $95^{\circ} \mathrm{C}$ for 10 seconds, $55^{\circ} \mathrm{C}$ for 10 seconds, and $72^{\circ} \mathrm{C}$ for 30 seconds. The dissolution curve was analyzed to determine the specificity of the real-time PCR amplification. The relative expression level of $h s a-m i R-181 a$ was calculated by the comparative cycle threshold method, with $U 6$ as the internal reference and expressed as the percentage change relative to untreated controls. Quantification of the relative expression levels of $h s a-m i R-181 a$ was achieved by the following formula: $2^{-\Delta \Delta \mathrm{Ct}}$, where $\Delta \Delta \mathrm{Ct}$ equals (Ct of hsa-miR-181a- $\mathrm{Ct}$ of U6) $)_{\text {experiment }}$ minus ( $\mathrm{Ct}$ of hsa-miR-181a- $\mathrm{Ct}$ of U6) control . $2^{-\Delta \Delta \mathrm{Ct}}$ was presented as the relative change of $h s a-m i R-181 a$ expression.

\section{Statistical analysis}

Data are expressed as the mean \pm standard deviation (SD). Statistical analysis was performed using one-way analysis of variance (ANOVA) followed by Tukey's multiple comparison to determine statistical significance among multiple groups. Student's $t$-test was used to compare the difference between two groups when appropriate. $P<0.05$ was considered as statistically significant.

\section{Results}

\section{Predicted targets of hsa-miR- I8Ia-5p and hsa-miR-I8 Ia-3p using various algorithms} Predicted targets of hsa-miR-18la-5p

Using DIANA microT v3.0, hsa-miR-181a-5p was predicted to regulate 522 targets when the threshold was set to 0.7 , including $A T P 1 B 1, B H L H E 40, C L A S P 1, C R E$ BRF, FBXO33, GLS, KLHL5, LRBA, MAP1B, MTF2, NMT2, PAPD5, REPS2, RLF, SESN3, SLC2A3, SLITRK1, TMEM131, TRIM2, ZFP90, ZNF268, ZNF441, ZNF527, ZNF570, ZNF704, ZNF717, ZNF781, ZNF788, ZNF799, ZNF846, etc (Table 3 and Table S2). Many of these targets regulate a number of important cellular processes, such as cell proliferation, cell death, cell division, mitosis, metabolism of glucose, lipids, nuclear acids, and amino acids, and transport. Among the predicted targets, 30 of the predicted targets (5.74\%) were cancer genes, including $A B I 1, A R I D 2$, ATM, BCL11B, BCL6, CARD11, CCDC6, CHN1, CREB1, GNAQ, HMGA2, LCP1, MAF, MAP2K1, MLLT10, MSI2, NOTCH2, NR4A3, PAX5, PBX1, PHOX2B, PMS1, PRDM1, PTEN, SS18L1, STAG2, TRIM33, WHSC1, WIF1, and XPO1 (Table 3 and Table S2).

miRanda-mirSVR predicted that hsa-miR-181a-5p could regulate 7,847 transcripts/targets (Table 3 and Table S3).
These included ZNF527, ZNF439, ZNF781, ZNF559, ZNF204P, BAZ2B, ZNF844, C15orf29, FBXO34, C5orf41, KIAA0528, ZNF594, EIF4A2, ZNF833, GATM, ZNF440, MARK1, OSBPL3, AP1G1, GABRA1, DDX3X, etc. Most of these genes regulate a number of important cellular processes, such as cell proliferation, cell death, division, mitosis, metabolism of glucose, lipids, nuclear acids, and amino acids, and transport. Notably, 223 genes from these predicted targets were cancer genes $(2.84 \%)$ (Table S4). These included $A B I 1, A B L 1, A B L 2, A K A P 9, A K T 2, A P C$, ARHGEF12, ARID2, ARNT, ATF1, ATM, ATP2B3, ATRX, BAP1, BCL2, BCL6, BCL7A, BCL9, BCOR, BCR, BRCA1, BTG1, C16orf75, CALR, CAMTA1, CANT1, CARD11, CASP8, CBFA2T3, CBL, CBLB, CCDC6, CCNE1, CD274, CDC73, CDH1, CDK6, CDX2, CEBPA, CHCHD7, CHN1, CLTC, CREB1, CREB3L2, CREBBP, CRTC3, CTNNB1, CYLD, DAXX, DDX10, DDX5, DEK, DICER1, DNM2, ECT2L, EGFR, EIF4A2, EML4, EPS15, ERG, ETV1, ETV6, EXT2, EZH2, FAM46C, FANCA, FANCD2, FANCF, FANCG, FAS, FBXO11, FGFR1, FGFR1OP, FGFR2, FGFR3, FLT3, FNBP1, FOXP1, FSTL3, FUS, GAS7, GATA2, GNAS, GOPC, H3F3B, HERPUD1, HEY1, HLF, HMGA2, HNRNPA2B1, HOOK3, HOXA11, HOXC11, HOXC13,IDH1,IGL@,IL2,IL21R,IL6ST,IL7R,IRF4, JAK2, JAZF1, JUN, KCNJ5, KDM5A, KDM5C, KDM6A, KDR, KIAA1549, KLK2, KRAS, LASP1, LCP1, LIFR, LMO1, LPP, MAF, MAFB, MALAT1, MALT1, MAP2K1, MAP2K4, MDM2, MDM4, MDS2, MET, MITF, MKL1, MLF1, MLH1, MLL, MLL3, MLLT10, MLLT3, MLLT4, MLLT6, MN1, MPL, MSH2, MSI2, MYB, MYCN, MYH11, NCOA1, NCOA2, NF1, NF2, NFE2L2, NFIB, NIN, NOTCH2, NPM1, NR4A3, NRAS, NSD1, NT5C2, NUP98, PAFAH1B2, PAX3, PAX5, PAX7, PBRM1, PBX1, PDE4DIP, PDGFRA, PDGFRB, PHF6, PHOX2B, PICALM, PIK3R1, PLAG1, PML, PMS1, PPARG, PRDM1, PTEN, PTPRC, RAC1, RAD21, RALGDS, RANBP17, RAP1GDS1, RB1, RNF43, RPL5, RUNX1, SDC4, SETBP1, SF3B1, SH2B3, SLC34A2, SMARCA4, SMARCE1, SRGAP3, SS18L1, STAT3, SUFU, SUZ12, SYK, TAF15, TAL2, TBL1XR1, TCF7L2, TCL6, TET2, TFRC, TMPRSS2, TOP1,TPM3,TRA@,TRIM27,TRIM33,TRRAP,TSC1, TSHR, U2AF1, UBR5, VTI1A, WHSC1, WIF1, YWHAE, and ZNF521 (Table S4).

miRDB predicted that 1,065 targets/transcripts were possibly regulated by hsa-miR-181a-5p (Table 3 and Table S5). These included PDE5A, ZNF439, PRTG, BRWD1, ZNF549, NFAT5, SH3TC2, OSBPL3, GFPT1, ZNF781, TNPO1, PAPD5, FIGN, S1PR1, TMEM87B, DDX3X, ZNF559, ZNF844, CLMN, GPR26, CTDSPL, ANKRD13C, FUT9, 


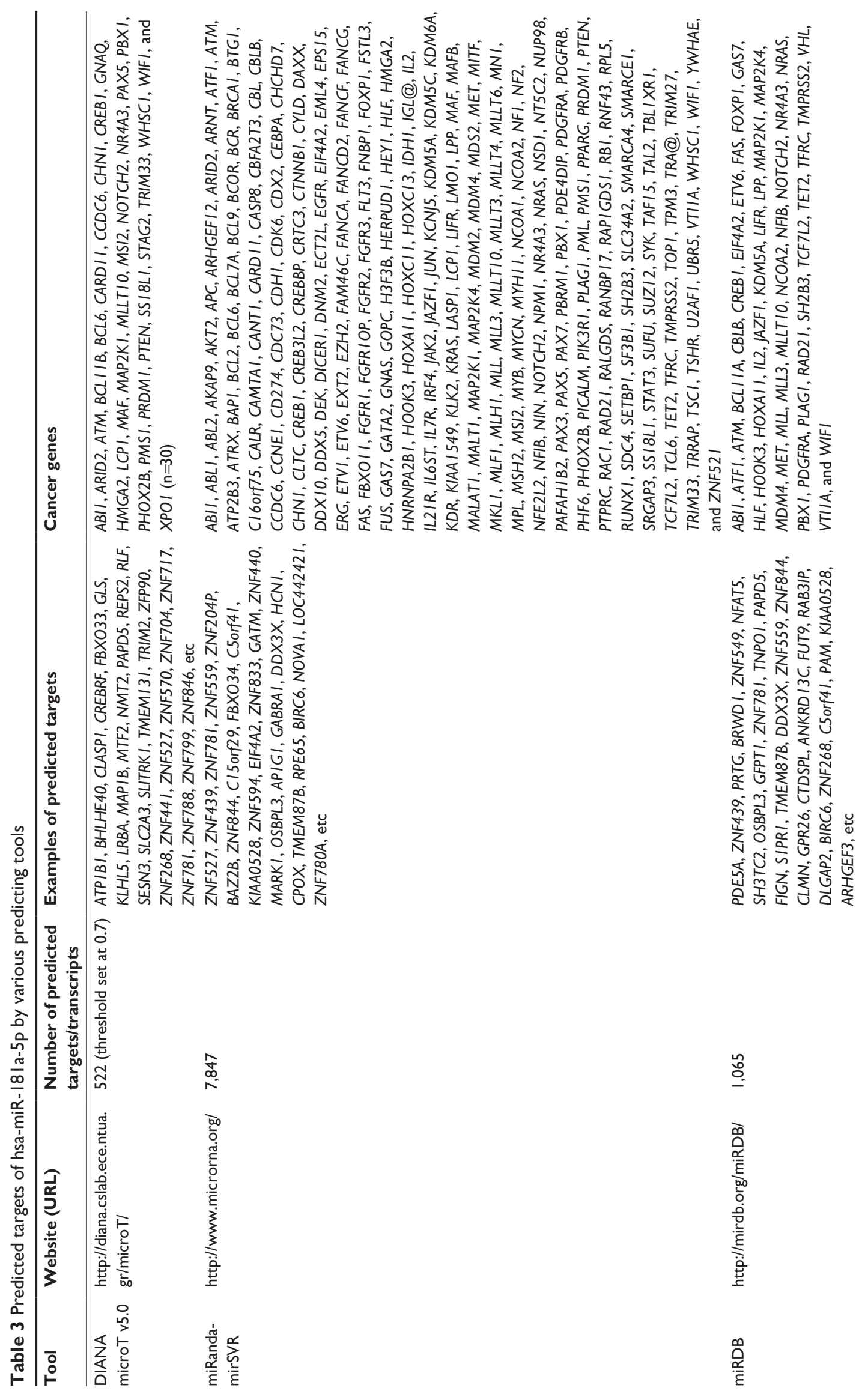




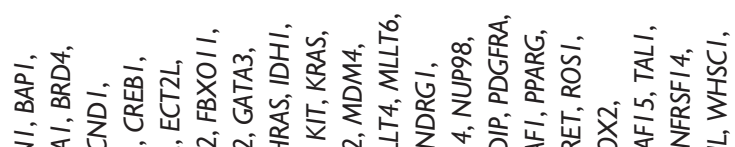

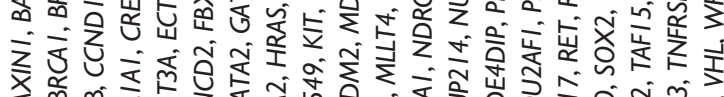

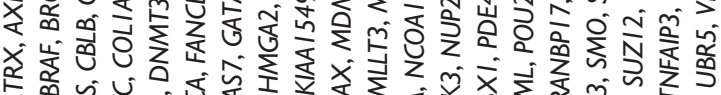

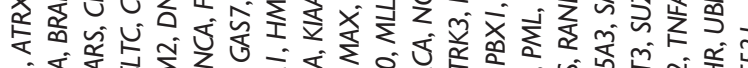

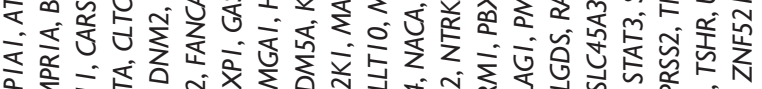

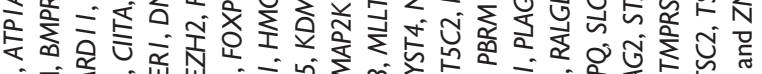

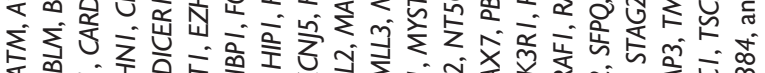

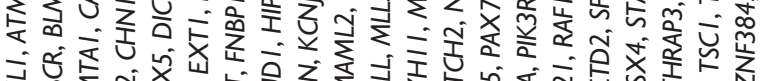

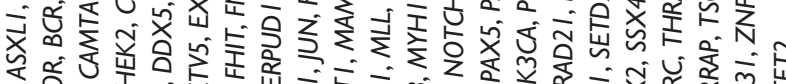

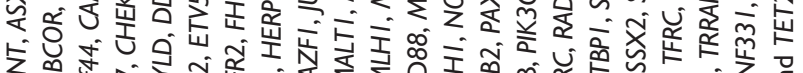

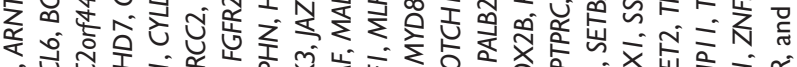

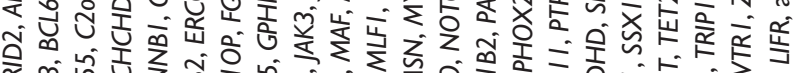

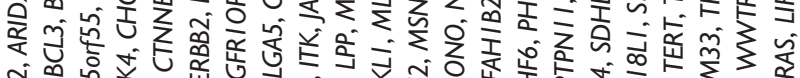

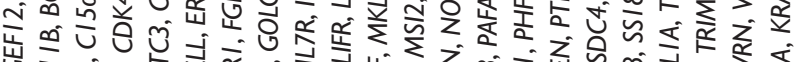

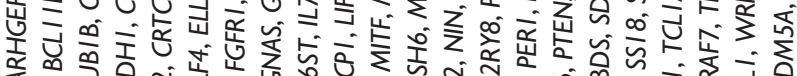

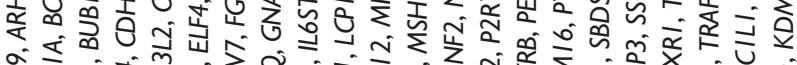

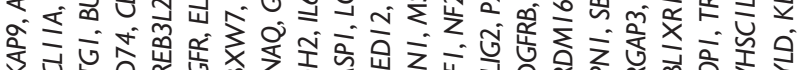

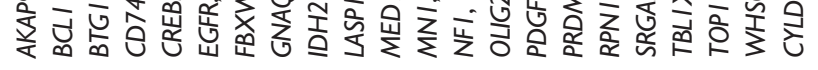

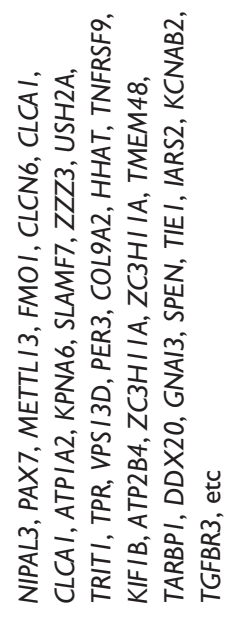

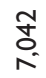

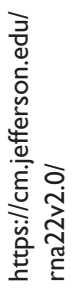

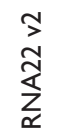

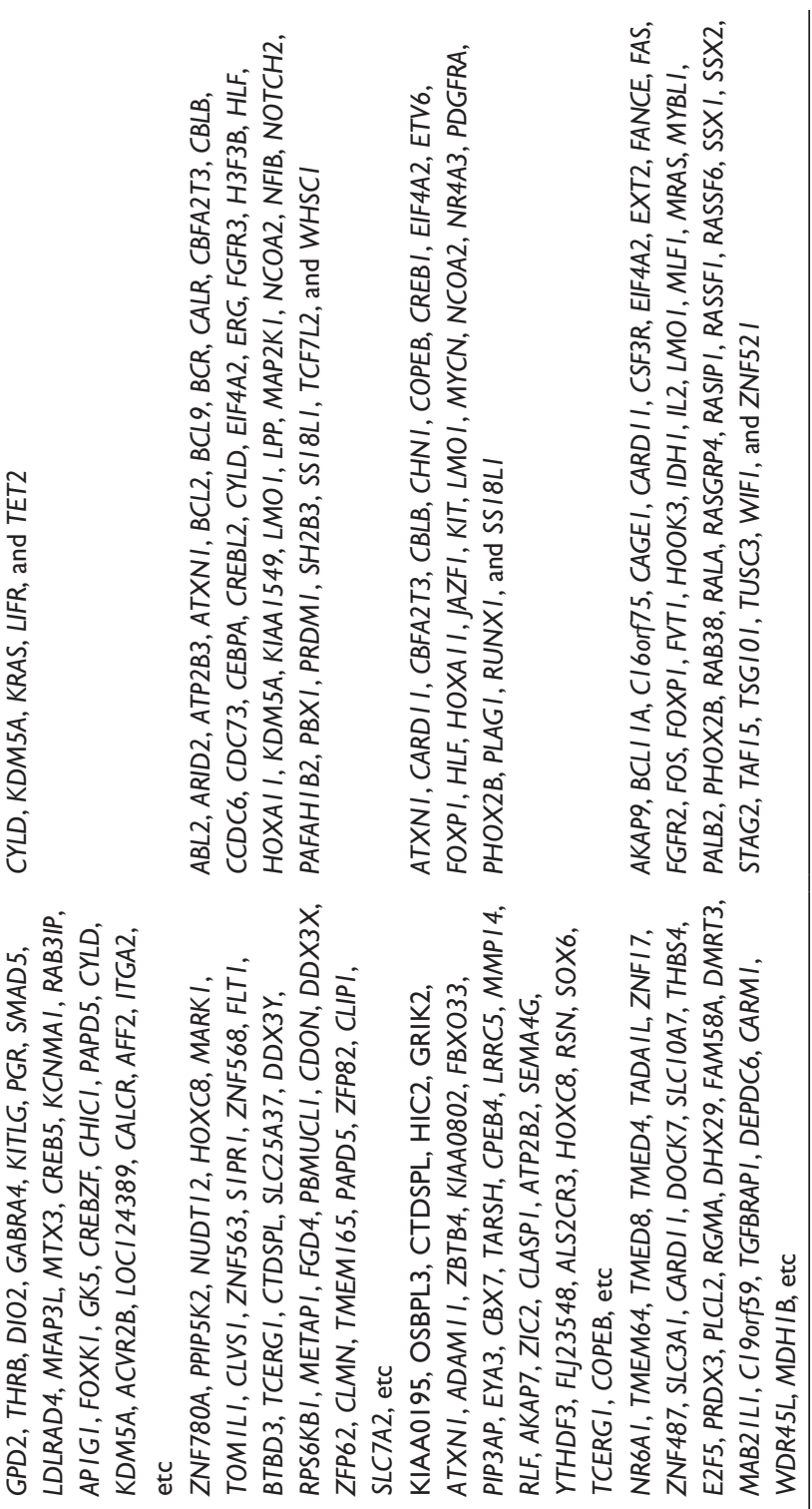

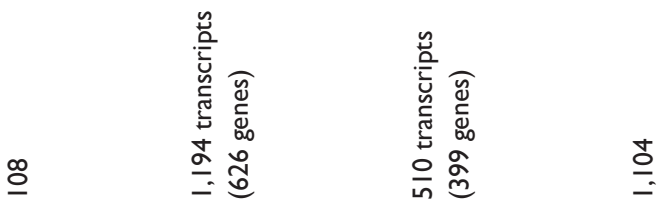
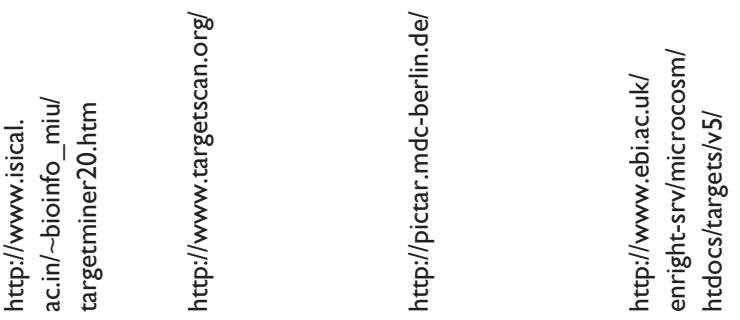

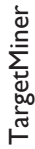

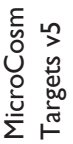


RAB3IP, DLGAP2, BIRC6, ZNF268, C5orf41, PAM, $K I A A 0528, A R H G E F 3$, etc. Many of the predicted targets play a role in the regulation of cell proliferation, cell cycle, apoptosis, energy, nuclear acid, and protein metabolism, signaling transduction, and transport. Among these targets, 43 targets were cancer genes (4.04\%). These included $A B I 1$, ATF1, ATM, BCL11A, CBLB, CREB1, EIF4A2, ETV6, FAS, FOXP1, GAS7, HLF, HOOK3, HOXA11, IL2, JAZF1, KDM5A, LIFR, LPP, MAP2K1, MAP2K4, MDM4, MET, MLL, MLL3, MLLT10, NCOA2, NFIB, NOTCH2, NR4A3, NRAS, PBX1, PDGFRA, PLAG1, RAD21, SH2B3, TCF7L2, TET2, TFRC, TMPRSS2, VHL, VTI1A, and WIF1 (Table 3 and Table S5).

Using RNA22 v2, 7,028 targets were predicted to be regulated by hsa-miR-181a-5p (Table 3 and Table S6). These included NIPAL3, PAX7, METTL13, FMO1, CLCN6, CLCA1, CLCA1, ATP1A2, KPNA6, SLAMF7, ZZZ3, USH2A, TRIT1, TPR, VPS13D, PER3, COL9A2, HHAT, TNFRSF9, KIF1B, ATP2B4, ZC3H11A, ZC3H11A, TMEM48, TARBP1, DDX20, GNAI3, SPEN, $T I E 1, I A R S 2, K C N A B 2, T G F B R 3$, etc. Many of the predicted targets play a role in the regulation of cell proliferation, cell cycle, apoptosis, energy, nuclear acid and protein metabolism, signaling transduction, and transport. Among the predicted targets, 211 genes were cancer genes (3.00\%) (Table S7). These included AKAP9, ARHGEF12, ARID2, ARNT, ASXL1, ATM, ATP1A1, ATRX, AXIN1, $B A P 1, B C L 11 A, B C L 11 B, B C L 3, B C L 6, B C O R, B C R$, $B L M, B M P R 1 A, B R A F, B R C A 1, B R D 4, B T G 1, B U B 1 B$, C15orf55, C2orf44, CAMTA1, CARD11, CARS, CBLB, CCND1, CD74, CDH1, CDK4, CHCHD7, CHEK2, CHN1, CIITA, CLTC, COL1A1, CREB1, CREB3L2, CRTC3, CTNNB1, CYLD, DDX5, DICER1, DNM2, DNMT3A, ECT2L, EGFR, ELF4, ELL, ERBB2, ERCC2, ETV5, EXT1, EZH2, FANCA, FANCD2, FBXO11, FBXW7, FGFR1, FGFR1OP, FGFR2, FHIT, FNBP1, FOXP1, GAS7, GATA2, GATA3, GNAQ, GNAS, GOLGA5, GPHN, HERPUD1, HIP1, HMGA1, HMGA2, HRAS, IDH1, IDH2, IL6ST, IL7R, ITK, JAK3, JAZF1, JUN, KCNJ5, KDM5A, KIAA1549, KIT, KRAS, LASP1, LCP1, LIFR, LPP, MAF, MALT1, MAML2, MAP2K1, MAX, MDM2, MDM4, MED12, MITF, MKL1, MLF1, MLH1, MLL, MLL3, MLLT10, MLLT3, MLLT4, MLLT6, MN1, MSH6, MSI2, MSN, MYD88, MYH11, MYST4, NACA, NCOA1, NDRG1, NF1, NF2, NIN, NONO, NOTCH1, NOTCH2, NT5C2, NTRK3, NUP214, NUP98, OLIG2, P2RY8, PAFAH1B2, PALB2, PAX5, PAX7, PBRM1, PBX1, PDE4DIP, PDGFRA, PDGFRB, PER1, PHF6, PHOX2B,
PIK3CA, PIK3R1, PLAG1, PML, POU2AF1, PPARG, PRDM16, PTEN, PTPN11, PTPRC, RAD21, RAF1, RALGDS, RANBP17, RET, ROS1, RPN1, SBDS, SDC4, SDHD, SETBP1, SETD2, SFPQ, SLC45A3, SMO, SOX2, SRGAP3, SS18, SS18L1, SSX1, SSX2, SSX4, STAG2, STAT3, SUZ12, TAF15, TAL1, TBL1XR1, TCL1A, TERT, TET2, TFRC, THRAP3, TMPRSS2, TNFAIP3, TNFRSF14, TOP1, TRAF7, TRIM33, TRIP11, TRRAP, TSC1, TSC2, TSHR, UBR5, VHL, WHSC1, WHSC1L1, WRN, WWTR1, ZNF331, ZNF384, and ZNF521 (Table S7).

TargetMiner predicted that 108 targets were regulated by hsa-miR-181a-5p, including GPD2, THRB, DIO2, GABRA4, KITLG, PGR, SMAD5, LDLRAD4, MFAP3L, MTX3, CREB5, KCNMA1, RAB3IP, AP1G1, FOXK1, GK5, CREBZF, CHIC1, PAPD5, CYLD, KDM5A, ACVR2B, LOC124389, CALCR, AFF2, ITGA2, etc (Table 3 and Table S8). Among these predicted targets, five were cancer genes, including CYLD, KDM5A, KRAS, LIFR, and TET2 (4.63\%) (Table 3 and Table S8).

TargetScan 6.2 only provided the predicted targets for the precursor hsa-miR-181a. It predicted that hsa-miR-181a could regulate 1,194 transcripts with conserved sites, with a total of 1,412 conserved sites and 626 poorly conserved sites (Table 3 and Table S9). Among these transcripts, 626 were functional genes. These included ZNF780A, PPIP5K2, NUDT12, HOXC8, MARK1, TOM1L1, CLVS1, ZNF563, S1PR1, ZNF568, FLT1, BTBD3, TCERG1, CTDSPL, SLC25A37, DDX3Y, RPS6KB1, METAP1, FGD4, PBMUCL1, CDON, DDX3X, ZFP62, CLMN, TMEM165, PAPD5, ZFP82, CLIP1, SLC7A2, etc. Many of the targets were involved in the regulation of cell proliferation, cell cycle, apoptosis, energy, nuclear acid, and protein metabolism, signaling transduction, and transport. Among the predicted targets, 36 were cancer genes $(5.75 \%)$. These included $A B L 2$, ARID2, ATP2B3, ATXN1, BCL2, BCL9, $B C R, C A L R, C B F A 2 T 3, C B L B, C C D C 6, C D C 73, C E B P A$, CREBL2, CYLD, EIF4A2, ERG, FGFR3, H3F3B, HLF, HOXA11, KDM5A, KIAA1549, LMO1, LPP, MAP2K1, NCOA2, NFIB, NOTCH2, PAFAH1B2, PBX1, PRDM1, SH2B3, SS18L1, TCF7L2, and WHSC1 (Table 3 and Table S9).

PicTar only provided predicted targets for the precursor hsa-miR-181a. It predicted that hsa-miR-181a could regulate 510 transcripts with 399 genes, including KIAA0195, OSBPL3, CTDSPL, HIC2, GRIK2, ATXN1, ADAM11, ZBTB4, KIAA0802, FBXO33, PIP3AP, EYA3, CBX7, TARSH, CPEB4, LRRC5, MMP14, RLF, AKAP7, ZIC2, CLASP1, ATP2B2, SEMA4G, YTHDF3, FLJ23548, 
ALS2CR3, HOXC8, RSN, SOX6, TCERG1, COPEB, etc (Table 3 and Table S10). Many of the predicted targets play a role in the regulation of cell proliferation, cell cycle, apoptosis, energy, nuclear acid, and protein metabolism, signaling transduction, and transport. Among the predicted targets, $23(5.76 \%)$ were cancer genes involved in the initiation, growth, and development and metastasis of cancer, including $A T X N 1, C A R D 11, C B F A 2 T 3, C B L B$, CHN1, COPEB, CREB1, EIF4A2, ETV6, FOXP1, HLF, HOXA11, JAZF1, KIT, LMO1, MYCN, NCOA2, NR4A3, PDGFRA, PHOX2B, PLAG1, RUNX1, and SS18L1 (Table 3 and Table S10).

MicroCosm Targets v5 predicted that 1,104 targets/ transcripts were likely regulated by hsa-miR-181a-5p (Table 3 and Table S11). These included NR6A1, TMEM64, TMED8, TMED4, TADA1L, ZNF17, ZNF487, SLC3A1, CARD11, DOCK7, SLC10A7, THBS4, E2F5, PRDX3, PLCL2, RGMA, DHX29, FAM58A, DMRT3, MAB21L1, C19orf59, TGFBRAP1, DEPDC6, CARM1, WDR45L, $M D H 1 B$, etc. Among the predicted targets, 37 (3.35\%) were cancer genes, including $A K A P 9, B C L 11 A, C 16$ orf75, CAGE1, CARD11, CSF3R, EIF4A2, EXT2, FANCE, FAS, FGFR2, FOS, FOXP1, FVT1, HOOK3, IDH1, IL2, LMO1, MLF1, MRAS, MYBL1, PALB2, PHOX2B, RAB38, RALA, RASGRP4, RASIP1, RASSF1, RASSF6, SSX1, SSX2, STAG2, TAF15, TSG101, TUSC3, WIF1, and ZNF521 (Table 3 and Table S11).

These results showed that the number of predicted targets of hsa-miR-181a-5p by the eight algorithms was very different, ranging from 108 to 7,847, with a mean of 2,424. Most of the predicted targets are involved in the regulation of cell proliferation, cell division, cell apoptosis, energy metabolism, amino acid, and nucleic acid metabolism, and transport, inflammation, redox homeostasis, and stress response. Many of the predicted targets are cancer genes, which participate in cancer initiation, development, growth, and metastasis. These cancer genes, including tumor suppressor genes and oncogenes, act as drivers or passengers in tumorigenesis. They are involved in various aspects of functions implicated in cancer initiation, development, and metastasis, including control of cell proliferation, apoptosis, signal transduction, transcription regulation, immunity, and defense.

\section{Predicted targets of hsa-miR-I8Ia-3p}

Using DIANA microT v3.0, hsa-miR-181a-3p was predicted to regulate 249 transcripts/targets when the threshold was set at 0.45 (Table 4 and Table S12). These included ETV1,
GGCT, ODZ1, NUB1, CPS1, AGPAT4, TRIO, LMO3, COL9A2, ELN, HEBP2, CYFIP2, MCOLN3, RC3H2, FLYWCH1, COL11A1, MRPS35, DGKA, LAPTM4A, IFT80, CLEC2D, AFF4, MARK2, SENP1, STX7, PDS5B, NKAIN1, PSMC5, SLC26A4, etc. Among these targets, 15 were cancer genes (6.12\%), including ASPSCR1, BCL11A, CACNA1D, CCND2, ELN, ETV1, EZH2, GATA3, HIP1, HRAS, MSI2, PIM1, TSHR, USP6, and WIF1 (Table 4 and Table S12).

miRanda-mirSVR predicted that hsa-miR-181a-3p could regulate 1,873 targets (Table 4 and Table S13). These genes included PMS2L2, COL27A1, SAE1, CNTNAP3B, FAM153B, GRIP2, NR4A1, RXRA, AES, POM121C, AFG3L1, XDH, XPA, MID1, AIRE, CTSK, HSD17B1,LOR, LTBP2, NEU1, AMPD3, AMT, APP, FUT2, etc. Most of these genes regulate a number of important cellular processes, such as cell proliferation, cell death, division, mitosis, metabolism of glucose, lipids, nuclear acids, and amino acids, and transport. Among the predicted targets, 61 were cancer genes $(3.26 \%)$. These included $A B I 2, A T M, A X I N 1, B C L 11 A, B C L 2, B T G 1$, CACNA1D, CASP8,CDH1, CDK12, CEBPA, CHCHD7, CREB1, CRTC3, DDB2, DDX10, DNM2, DUX4, EBF1, EIF4A2, ELL, ETV6, EZH2, FOXP1, GAS7, GATA3, GNA11, GNAS, HIP1, LMO1, LPP, MALAT1, MAX, MKL1, MLL3, MLLT1, MN1, MSI2, NF1, PAFAH1B2, PAX5, PAX7, PDE4DIP, PER1, PIM1, PML, PTEN, RANBP17, RPL10, SEPT6, SET, SETBP1, SMARCE1, SS18L1, TAL1, TBL1XR1, TCL6, TET2, TP53, TSHR, and TTL (Table 4 and Table S14).

miRDB predicted that 22 targets were possibly regulated by hsa-miR-181a-3p (Table 4 and Table S15). These included ALDH18A1, ATP13A4, ALDH6A1, C16orf57, NIPA2, RIBC1, SLC20A2, C14orf28, CFL2, ZNF3, RHOBTB1, H1F0, AP1S3, ARL4A, RBM22, MIER1, ACTR3, CLEC2D, $A F F 2, A C A P 2, R A B G E F 1$, and KCTD12. These targets play a role in the regulation of cell proliferation, cell cycle, apoptosis, energy, nuclear acid, and protein metabolism, signaling transduction, and transport. However, none of the predicted targets were cancer genes.

Using RNA22 v2, 5,142 transcripts/2,718 genes were predicted to be regulated by hsa-miR-181a-3p (Table 4 and Table S16). These included CFH, TTC22, FMO1, ATP1A2, VPS13D, COL9A2, UTS2, LAMC2, PIGV, PTPRU, COL11A1, DDX20,WDR3, YBX1, ASPM, LRRC40, EPHA8, ARHGEF10L, RASAL2, PLXNA2, RAP1GAP, PPP1R12B, SDF4, TP73, NKAIN1, WDR47, OVGP1, SLC25A24, EPS15, $P O M G N T 1$, etc. Many of the predicted targets play a role 


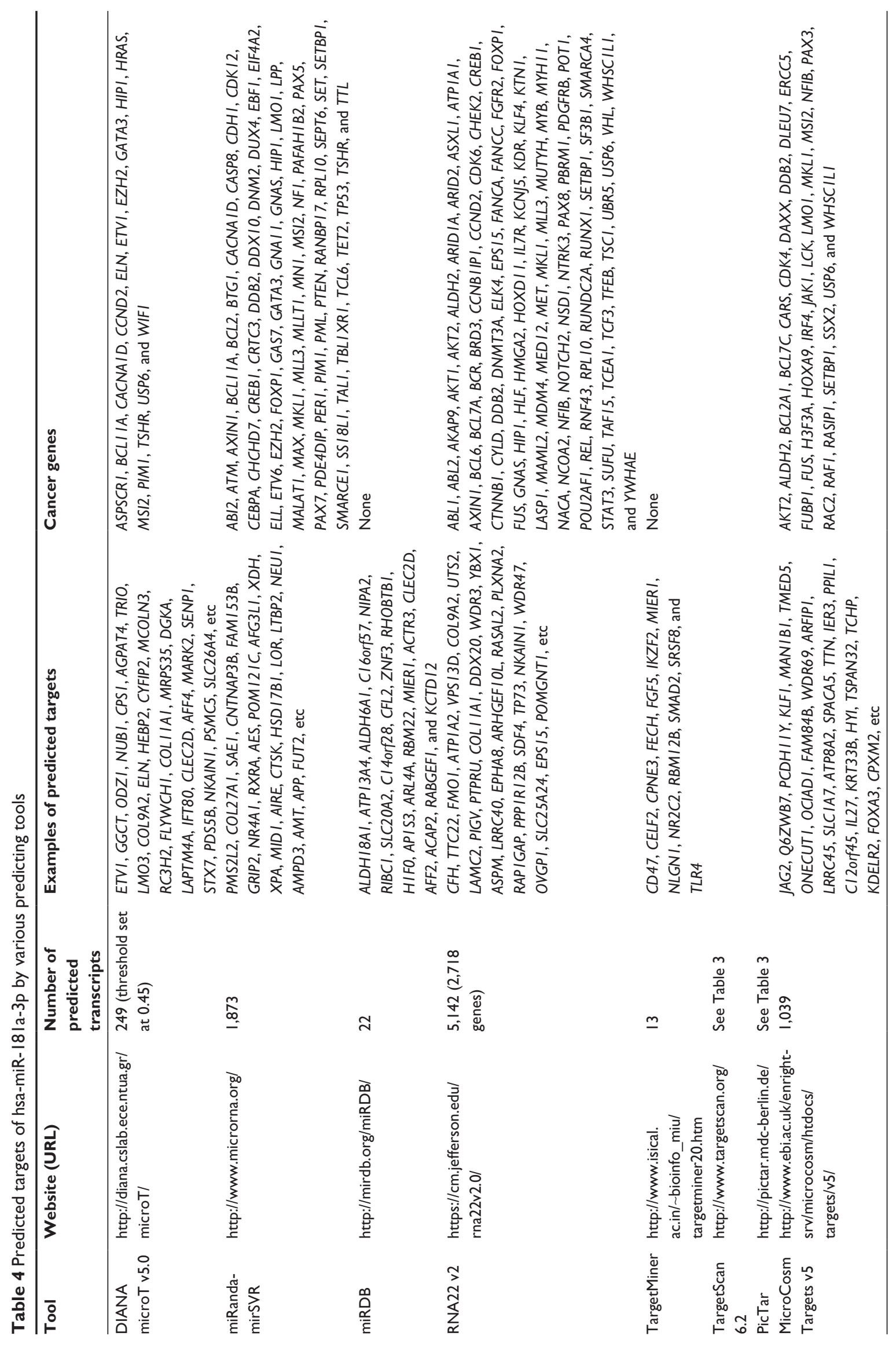


in the regulation of cell proliferation, cell cycle, apoptosis, energy, nuclear acid, and protein metabolism, signaling transduction, and transport. Among these targets, 82 were cancer genes (3.02\%), including $A B L 1, A B L 2, A K A P 9, A K T 1, A K T 2$, ALDH2, ARID1A, ARID2, ASXL1, ATP1A1, AXIN1, BCL6, BCL7A, BCR, BRD3, CCNB1IP1, CCND2, CDK6, CHEK2, CREB1, CTNNB1, CYLD, DDB2, DNMT3A, ELK4, EPS15, FANCA, FANCC, FGFR2, FOXP1, FUS, GNAS, HIP1, HLF, HMGA2, HOXD11, IL7R, KCNJ5, KDR, KLF4, KTN1, LASP1, MAML2, MDM4, MED12, MET, MKL1, MLL3, MUTYH, MYB, MYH11, NACA, NCOA2, NFIB, NOTCH2, NSD1, NTRK3, PAX8, PBRM1, PDGFRB, POT1, POU2AF1, REL, RNF43, RPL10, RUNDC2A, RUNX1, SETBP1, SF3B1, SMARCA4, STAT3, SUFU, TAF15, TCEA1, TCF3, TFEB, TSC1, UBR5, USP6, VHL, WHSC1L1, and YWHAE (Table 4 and Table S16).

TargetMiner predicted that 13 targets were regulated by hsa-miR-181a-3p, including CD47, CELF2, CPNE3, FECH, FGF5, IKZF2, MIER1, NLGN1, NR2C2, RBM12B, $S M A D 2, S R S F 8$, and TLR4 (Table 4 and Table S17). None of the predicted targets was a cancer gene.

TargetScan 6.2 predicted that hsa-miR-181a could regulate 626 targets (Tables 3 and 4). In PicTar, only hsa-miR181 a could be searched. It probably regulated 510 transcripts with 399 genes (Tables 3 and 4).

MicroCosm Targets v5 predicted that 1,039 targets were likely regulated by hsa-miR-181a-3p (Table 4 and Table S18). These included $J A G 2, Q 6 Z W B 7, P C D H 11 Y, K L F 1$, MAN1B1, TMED5, ONECUT1, OCIAD1, FAM84B, WDR69, ARFIP1, LRRC45, SLC1A7, ATP8A2, SPACA5, TTN, IER3, PPIL1, C12orf45, IL27, KRT33B, HYI, TSPAN32, TCHP, KDELR2, FOXA3, CPXM2, etc. Among the predicted targets, 29 were cancer genes (2.79\%), including $A K T 2$, ALDH2, BCL2A1, BCL7C, CARS, CDK4, DAXX, DDB2, DLEU7, ERCC5, FUBP1, FUS, H3F3A, HOXA9, IRF4, JAK1, LCK, LMO1, MKL1, MSI2, NFIB, PAX3, RAC2, RAF1, RASIP1, SETBP1, SSX2, USP6, and WHSC1L1 (Table 4 and Table S18).

These results demonstrated that the number of predicted targets of hsa-miR-181a-3p by the eight tools was very different, ranging from 13 to 5,142, with a mean of 1,184 . Most of the predicted targets are involved in the regulation of cell proliferation, cell division, cell apoptosis, energy metabolism, amino acid and nucleic acid metabolism, and transport, inflammation, redox homeostasis, and stress response. Many of the predicted targets are cancer genes which participate in cancer initiation, development, growth, and metastasis.

\section{Predicted targets of hsa-miR-I8Ia by miRWALK} miRWALK provides information on predicted targets from ten algorithms. When only miRWALK was chosen, where only one longest binding site was set per miRNA per mRNA, 3,762 transcripts were predicted to be regulated by hsa-miR-181a (Table S19). These included AASS, ABCB11, ACN9, ACTA2, ADAM28, BAG2, BCL11A, BCL6B, BTRC, CARD11, CCR3, CDK8,CDKN3, CIT, CNOT1, DAD1, DCN, DKC1, DR1, EHF, ESCO2, FBN2, FUT1, GALNT3, GCS1, GPC5, HK2, HSPB3, IL2, IL25, IVD, KCNG3, KIF2C, LACE1, MAEL, MAOA, MLL5, NCL, NOVA1, OCA2, PAG1, PROCR, RAD21, RFC3, RNF6, SELT, SIX2, TAF15, etc. Among these transcripts, 1,436 were functional genes (Table S20).

miRWALK also provided a summarized table that included all targets predicted to be regulated by hsa-miR$181 \mathrm{a}$ by the ten algorithms. In total, there were 17,395 transcripts that would be regulated by hsa-miR-181a (Table S21). Only 24 genes were predicted to be the targets of hsa-miR-181a by all the algorithms or at least nine algorithms. These included RNF145, TCERG1, SIRT1, SS18L1, NR6A1, FOXP1, GLS, HOXA11, SMAD7, MAP1B, INOC1, CDKN2AIP, LRRN1, TGFBI, YWHAG, C17orf39, CHD9, NAT13, C6orf62, ACVR2A, DCLK1, NMT2, NPEPPS, and KIAA0195. There were 102 targets that were predicted to be regulated by hsa-miR-181a by eight algorithms, including CDH13, PDIA6, IPO8, IGF2BP2, SLITRK1, SSX2IP, COL16A1, ADM, ZNF800, ADAMTS18, DPYSL2, E2F5, EIF4A2, EN2, ETV6, ACSL1, FBN2, C7orf41, FKBP1A, BTBD3, KIAA0423, HIC2, KANK1, HISPPD1, SYNE1, ZNF281, FOS, LEMD3, BRD1, OSBPL3, EPC2, GAPVD1, GATA6, NPTN, ATP11C, KLF15, LRP12, BAZ2B, HLF, HOXA1, ID4, JARID2, KPNA1, etc. There were 191, 778, 1,615 , and 3,168 targets that were predicted to be regulated by hsa-miR-181a by seven, six, five, and four algorithms, respectively.

\section{Validated targets of hsa-miR-I8Ia-5p based on TarBase}

Based on TarBase, 211 targets of hsa-miR-181a-5p have been validated with experimental evidence (Table 5). These included ACOT12, ALG10B, AMMECR1, ANKRD1, ANKRD13C, ARF6, ARHGAP11A, ARL6IP1, ARL6IP6, ATF7IP2, ATG10, ATM, ATP6V0E1, BAG2, BCL2, BDNF, BPGM, BRIX1, BRMS1L, BTBD3, Clorf109, Clorf43, C1QTNF9, C8A, CBX3, CCDC6, CCDC82, CCND1, $C C N G 1$, etc. Among these validated targets, only eleven of them are cancer genes (5.21\%), including ATM, BCL2, 
Table 5 Targets of hsa-miR-18Ia-5p with experimental evidence based on TarBase 6.0

\begin{tabular}{|c|c|c|c|c|c|}
\hline Gene symbol & $\begin{array}{l}\text { Accession } \\
\text { number }\end{array}$ & Full name & Alias & Function & $\begin{array}{l}\text { Cancer } \\
\text { gene }\end{array}$ \\
\hline ACOTI2 & NM_I30767 & Acyl-CoA thioesterase 12 & $\begin{array}{l}\text { CACH-I, Cach, STARDI5, } \\
\text { THEAL }\end{array}$ & $\begin{array}{l}\text { Hydrolyzes acetyl-CoA to acetate } \\
\text { and CoA }\end{array}$ & \\
\hline$A L G I O B$ & NM_001013620 & $\alpha$-I,2-Glucosyltransferase & ALGI0, KCRI & $\begin{array}{l}\text { Transfers glucose from dolichyl } \\
\text { phosphate glucose onto the } \\
\text { lipid-linked oligosaccharide Glc(2) } \\
\text { Man(9)GlcNAc(2)-PP-Dol }\end{array}$ & \\
\hline AMMECRI & NM_00I025580 & $\begin{array}{l}\text { Alport syndrome, mental } \\
\text { retardation, midface hypoplasia } \\
\text { and elliptocytosis chromosomal } \\
\text { region gene I }\end{array}$ & RPI3-360B22.I, AMMERCI & & \\
\hline ANKRDI & NM_01439I & $\begin{array}{l}\text { Ankyrin repeat domain I (cardiac } \\
\text { muscle) }\end{array}$ & $\begin{array}{l}\text { ALRP, C-193, CARP, } \\
\text { CVARP, MCARP, } \\
\text { bA320FI5.2 }\end{array}$ & $\begin{array}{l}\text { Plays an important role in } \\
\text { endothelial cell activation }\end{array}$ & \\
\hline ANKRD /3C & NM_030816 & Ankyrin repeat domain I3C & RP4-677HI5.5, dJ677HI5.3 & & \\
\hline ARF6 & NM_001663 & ADP-ribosylation factor 6 & & Involved in protein trafficking & \\
\hline ARHGAPIIA & NM_00I 286479 & $\begin{array}{l}\text { Rho GTPase activating } \\
\text { protein IIA }\end{array}$ & $\begin{array}{l}\text { RPII-I000B6.5, GAP } \\
(I-12)\end{array}$ & GTPase activator activity & \\
\hline ARLGIPI & NM_0I5I6I & $\begin{array}{l}\text { ADP-ribosylation factor-like } 6 \\
\text { interacting protein I }\end{array}$ & $\begin{array}{l}\text { AIPI, ARL6IP, ARMER, } \\
\text { SPG6I }\end{array}$ & $\begin{array}{l}\text { May be involved in protein } \\
\text { transport, membrane trafficking, or } \\
\text { cell signaling during hematopoietic } \\
\text { maturation }\end{array}$ & \\
\hline ARL6IP6 & NM_022989 & $\begin{array}{l}\text { ADP-ribosylation factor-like } 6 \\
\text { interacting protein } 6\end{array}$ & $\begin{array}{l}\text { RP23-265NI0.I, } \\
\text { 23I0057C0IRik, } \\
\text { 26I0529A I I Rik, Aip-6 }\end{array}$ & & \\
\hline ATF7IP2 & NM_00I256I60 & $\begin{array}{l}\text { Activating transcription factor } 7 \\
\text { interacting protein } 2\end{array}$ & MCAF2 & $\begin{array}{l}\text { Recruiter that couples } \\
\text { transcriptional factors to general } \\
\text { transcription apparatus and thereby } \\
\text { modulates transcription regulation } \\
\text { and chromatin formation }\end{array}$ & \\
\hline ATGIO & NM_001 I31028 & Autophagy related 10 & $\begin{array}{l}\text { PPI26I6, APGI0, APGIOL, } \\
\text { PPI } 2616\end{array}$ & Plays a role in autophagy & \\
\hline ATM & NM_00005I & ATM serine/threonine kinase & $\begin{array}{l}\text { ATI, ATA, ATC, ATD, } \\
\text { ATDC, ATE, TELI, TELOI }\end{array}$ & Serine/threonine protein kinase & Yes \\
\hline ATP6VOEI & NM_003945 & $\begin{array}{l}\text { ATPase, } \mathrm{H}^{+} \text {transporting, } \\
\text { lysosomal } 9 \mathrm{kDa} \text {, } \mathrm{V} 0 \text { subunit el }\end{array}$ & $\begin{array}{l}\text { ATP6H, ATP6V0E, M9.2, } \\
\text { Vma2I, Vma2IP }\end{array}$ & $\begin{array}{l}\text { Vacuolar ATPase is responsible for } \\
\text { acidifying a variety of intracellular } \\
\text { compartments in eukaryotic cells }\end{array}$ & \\
\hline BAG2 & NM_004282 & BCL2-associated athanogene 2 & $\begin{array}{l}\text { RP3-496NI7.2, BAG-2, } \\
\text { dJ4I7II. } 2\end{array}$ & $\begin{array}{l}\text { Inhibits the chaperone activity } \\
\text { of HSP70/HSC70 by promoting } \\
\text { substrate release }\end{array}$ & \\
\hline$B C L 2$ & NM_000633 & B-cell CLL/lymphoma 2 & $\mathrm{Bcl}-2, \mathrm{PPPIR} 50$ & Suppresses apoptosis & Yes \\
\hline$B D N F$ & NM_00II43805 & Brain-derived neurotrophic factor & ANON2, BULN2 & $\begin{array}{l}\text { Promotes the survival of neuronal } \\
\text { populations }\end{array}$ & \\
\hline BPGM & NM_00I293085 & 2,3-Bisphosphoglycerate mutase & DPGM & $\begin{array}{l}\text { Plays a major role in regulating } \\
\text { hemoglobin oxygen affinity }\end{array}$ & \\
\hline$B R I X I$ & NM_01832I & $\begin{array}{l}\text { Biogenesis of ribosomes, } \\
\text { homolog (S. cerevisiae) }\end{array}$ & $\mathrm{BRIX}, \mathrm{BXDC2}$ & $\begin{array}{l}\text { Required for biogenesis of the } 60 \mathrm{~S} \\
\text { ribosomal subunit }\end{array}$ & \\
\hline$B R M S I L$ & NM_032352 & $\begin{array}{l}\text { Breast cancer metastasis- } \\
\text { suppressor I-like }\end{array}$ & BRMSI & $\begin{array}{l}\text { Involved in the } \mathrm{HDACl} \text {-dependent } \\
\text { transcriptional repression activity }\end{array}$ & \\
\hline BTBD3 & NM_00I 282550 & BTB (POZ) domain containing 3 & RP4-742J24.3, dJ742J24.I & $\begin{array}{l}\text { Acts as a key regulator of } \\
\text { dendritic field orientation during } \\
\text { development of sensory cortex }\end{array}$ & \\
\hline Clorflo9 & NM_017850 & $\begin{array}{l}\text { Chromosome I open reading } \\
\text { frame } 109\end{array}$ & & & \\
\hline Clorf43 & NM_001098616 & $\begin{array}{l}\text { Chromosome I open reading } \\
\text { frame } 43\end{array}$ & $\begin{array}{l}\text { HSPC0I2, NICE-3, } \\
\text { NS5ATP4, S863-3 }\end{array}$ & & \\
\hline CIQTNF9 & NM_I83175 & $\begin{array}{l}\mathrm{Clq} \text { and tumor necrosis factor } \\
\text { related protein } 9\end{array}$ & $\begin{array}{l}\text { 9130217G22Rik, CTRP9, } \\
\text { Ciqtnf9 }\end{array}$ & $\begin{array}{l}\text { Activates AMPK, AKT, and p44/42 } \\
\text { MAPK signaling pathways }\end{array}$ & \\
\hline
\end{tabular}


Table 5 (Continued)

\begin{tabular}{|c|c|c|c|c|c|}
\hline Gene symbol & $\begin{array}{l}\text { Accession } \\
\text { number }\end{array}$ & Full name & Alias & Function & $\begin{array}{l}\text { Cancer } \\
\text { gene }\end{array}$ \\
\hline $\mathrm{CBA}$ & NM_000562 & $\begin{array}{l}\text { Complement component } 8, \alpha \\
\text { polypeptide }\end{array}$ & & $\begin{array}{l}\text { C8 is a constituent of the } \\
\text { membrane attack complex }\end{array}$ & \\
\hline$C B \times 3$ & NM_007276 & Chromobox homolog 3 & $\begin{array}{l}\text { HECH, HPI-GAMMA, } \\
\text { HPIHs- } \gamma\end{array}$ & $\begin{array}{l}\text { Involved in transcriptional silencing } \\
\text { in heterochromatin-like complexes }\end{array}$ & \\
\hline$C C D C 6$ & NM_005436 & Coiled-coil domain containing 6 & $\begin{array}{l}\text { DIOSI70, H4, PTC, TPC, } \\
\text { TSTI }\end{array}$ & Functions as a tumor suppressor & Yes \\
\hline CCDC82 & NM_024725 & Coiled-coil domain containing 82 & HT025, HSPC048 & & \\
\hline CCNDI & NM_053056 & Cyclin DI & $\begin{array}{l}\text { BCLI, DI IS287E, PRADI, } \\
\text { U2IB3I }\end{array}$ & $\begin{array}{l}\text { Essential for the control of the cell } \\
\text { cycle at the GI/S (start) transition }\end{array}$ & Yes \\
\hline CCNGI & NM_004060 & Cyclin GI & CCNG & May play a role in growth regulation & \\
\hline CD46 & NM_002389 & $\begin{array}{l}\text { CD } 46 \text { molecule, complement } \\
\text { regulatory protein }\end{array}$ & $\begin{array}{l}\text { AHUS2, MCP, MICI0, TLX, } \\
\text { TRA2.10 }\end{array}$ & $\begin{array}{l}\text { Acts as a cofactor for complement } \\
\text { factor I }\end{array}$ & \\
\hline$C D K N / B$ & NM_004064 & $\begin{array}{l}\text { Cyclin-dependent kinase inhibitor } \\
\text { IB (p27, KipI) }\end{array}$ & $\begin{array}{l}\text { CDKN4, KIPI, MENIB, } \\
\text { MEN4, P27KIPI }\end{array}$ & $\begin{array}{l}\text { Important regulator of cell cycle } \\
\text { progression }\end{array}$ & \\
\hline $\operatorname{CD} \times 2$ & NM_00I265 & Caudal type homeobox 2 & $\mathrm{CDX}-3, \mathrm{CDX} 3$ & $\begin{array}{l}\text { Involved in the transcriptional reg- } \\
\text { ulation of multiple genes expressed } \\
\text { in the intestinal epithelium }\end{array}$ & Yes \\
\hline CEP97 & NM_024548 & Centrosomal protein $97 \mathrm{kDa}$ & 28I0403B08Rik, LRRIQ2 & $\begin{array}{l}\text { Collaborates with cep I I0, being } \\
\text { involved in the suppression of a } \\
\text { cilia assembly program }\end{array}$ & \\
\hline CFI & NM_000204 & Complement factor I & $\begin{array}{l}\text { AHUS3, ARMDI3, C3BINA, } \\
\text { C3b-INA, FI, IF, KAF }\end{array}$ & $\begin{array}{l}\text { Responsible for cleaving the } \\
\alpha \text {-chains of } \mathrm{C} 4 \mathrm{~b} \text { and } \mathrm{C} 3 \mathrm{~b} \text { in } \\
\text { the presence of the cofactors } \\
\text { C4-binding protein and factor } \mathrm{H} \text {, } \\
\text { respectively }\end{array}$ & \\
\hline CHDI & NM_00I 270 & $\begin{array}{l}\text { Chromodomain helicase DNA } \\
\text { binding protein I }\end{array}$ & & $\begin{array}{l}\text { Sequence-selective DNA-binding } \\
\text { protein }\end{array}$ & \\
\hline CHLI & NM_00I253387 & Cell adhesion molecule LI-like & CALL, LICAM2 & $\begin{array}{l}\text { Plays a role in nervous system } \\
\text { development and in synaptic } \\
\text { plasticity }\end{array}$ & \\
\hline CHRFAM7A & NM_I39320 & $\begin{array}{l}\text { CHRNA7 (cholinergic receptor, } \\
\text { nicotinic, } \alpha 7 \text {, exons } 5-10 \text { ) and } \\
\text { FAM7A (family with sequence } \\
\text { similarity 7A, exons A-E) fusion }\end{array}$ & $\begin{array}{l}\text { CHRNA7, CHRNA7-DRI, } \\
\text { D-I0 }\end{array}$ & $\begin{array}{l}\text { Extracellular ligand-gated ion } \\
\text { channel activity }\end{array}$ & \\
\hline CLUAPI & NM_0I504I & Clusterin associated protein I & CFAP22, FAP22 & $\begin{array}{l}\text { May play a role in cell proliferation } \\
\text { or apoptosis }\end{array}$ & \\
\hline COL27AI & NM_032888 & Collagen, type XXVII, $\alpha$ I & RPII-82II.I & $\begin{array}{l}\text { Plays a role during the calcification } \\
\text { of cartilage and the transition of } \\
\text { cartilage to bone }\end{array}$ & \\
\hline COPS2 & NM_00II43887 & COP9 signalosome subunit 2 & $\begin{array}{l}\text { ALIEN, CSN2, SGN2, } \\
\text { TRIPI5 }\end{array}$ & $\begin{array}{l}\text { Involved in various cellular and } \\
\text { developmental processes }\end{array}$ & \\
\hline CST5 & NM_001900 & Cystatin D & & Cysteine proteinase inhibitor & \\
\hline CXorfI & NM_004709 & Transmembrane protein 257 & CXorfI & & \\
\hline$D 3 R$ & NM_000796.5 & DRD3 & D3DR; ETMI; FETI & $\begin{array}{l}\text { Associated with cognitive, } \\
\text { emotional, and endocrine functions }\end{array}$ & \\
\hline DCP2 & NM_00I242377 & Decapping mRNA 2 & NUDT20 & $\begin{array}{l}\text { Necessary for the degradation of } \\
\text { mRNAs }\end{array}$ & \\
\hline DCSTI & NM_00II43687 & DC-STAMP domain containing I & RPII-307CI2.10-003 & Protein and zinc ion binding & \\
\hline DDIT4 & NM_019058 & $\begin{array}{l}\text { DNA-damage-inducible } \\
\text { transcript } 4\end{array}$ & $\begin{array}{l}\text { RPI I-442H2I.I, Dig2, } \\
\text { REDD-I, REDDI }\end{array}$ & $\begin{array}{l}\text { Inhibits cell growth by regulating } \\
\text { the frapl pathway upstream of the } \\
\text { tscl-tsc2 complex and downstream } \\
\text { of Aktl }\end{array}$ & \\
\hline DNAJC7 & NM_00II44766 & $\begin{array}{l}\text { Dnal (HSP40) homolog, } \\
\text { subfamily C, member } 7\end{array}$ & DJII, DJC7, TPR2, TTC2 & $\begin{array}{l}\text { Acts as co-chaperone regulating } \\
\text { the molecular chaperones HSP70 } \\
\text { and HSP90 in folding of steroid } \\
\text { receptors }\end{array}$ & \\
\hline
\end{tabular}


Table 5 (Continued)

\begin{tabular}{|c|c|c|c|c|c|}
\hline Gene symbol & $\begin{array}{l}\text { Accession } \\
\text { number }\end{array}$ & Full name & Alias & Function & $\begin{array}{l}\text { Cancer } \\
\text { gene }\end{array}$ \\
\hline DSCR8 & NM_032589 & $\begin{array}{l}\text { Down syndrome critical region } \\
\text { gene } 8\end{array}$ & $\begin{array}{l}\text { C2Iorf65, CT25.Ia, } \\
\text { CT25.Ib, MMA-I, MMA-Ia, } \\
\text { MMA-Ib, MMAI, MTAG2 }\end{array}$ & & \\
\hline EIFI & NM_00580I & $\begin{array}{l}\text { Eukaryotic translation initiation } \\
\text { factor I }\end{array}$ & $\begin{array}{l}\text { AI2I, EIF-IA, ISOI, SUII, } \\
\text { EIFI }\end{array}$ & $\begin{array}{l}\text { Necessary for scanning and } \\
\text { involved in initiation site selection }\end{array}$ & \\
\hline $\mathrm{EIF} 2 \mathrm{Cl}$ & NM_012199 & $\begin{array}{l}\text { Argonaute RISC catalytic } \\
\text { component I }\end{array}$ & $\begin{array}{l}\text { RP4-789DI7.I, EIF2C, } \\
\text { AGOI, GERP95, Q99 }\end{array}$ & $\begin{array}{l}\text { Required for RNA-mediated gene } \\
\text { silencing }\end{array}$ & \\
\hline EIF2C3 & NM_024852 & $\begin{array}{l}\text { Argonaute RISC catalytic } \\
\text { component } 3\end{array}$ & AGO3 & $\begin{array}{l}\text { Required for RNA-mediated gene } \\
\text { silencing }\end{array}$ & \\
\hline ELAVLI & NM_001419 & ELAV like RNA binding protein I & ELAVI, HUR, Hua, MelG & $\begin{array}{l}\text { Binds avidly to the AU-rich } \\
\text { element in FOS and IL3/ } \\
\text { interleukin-3 mRNAs }\end{array}$ & \\
\hline ENAH & NM_00I008493 & Enabled homolog & $\begin{array}{l}\text { RPII-496NI2.7, ENA, } \\
\text { MENA, NDPPI }\end{array}$ & $\begin{array}{l}\text { Ena/VASP proteins are actin- } \\
\text { associated proteins involved in a } \\
\text { range of processes dependent on } \\
\text { cytoskeleton remodeling and cell } \\
\text { polarity }\end{array}$ & \\
\hline EP300 & NM_00I429 & EIA binding protein $\mathrm{p} 300$ & $\begin{array}{l}\text { RPI-85FI8.I, KAT3B, } \\
\text { RSTS2, p } 300\end{array}$ & $\begin{array}{l}\text { Functions as HAT and regulates } \\
\text { transcription via chromatin } \\
\text { remodeling }\end{array}$ & Yes \\
\hline EPHA5 & NM_00I28I765 & EPH receptor A5 & $\begin{array}{l}\text { CEK7, EHK-I, EHKI, EK7, } \\
\text { HEK7, TYRO4 }\end{array}$ & $\begin{array}{l}\text { Receptor for members of the } \\
\text { ephrin-A family }\end{array}$ & \\
\hline ESRI & NM_000I25 & Estrogen receptor I & $\begin{array}{l}\text { RPI-I30E4.I, ER, ESR, } \\
\text { ESRA, ESTRR, Era, NR3AI }\end{array}$ & Nuclear hormone receptor & \\
\hline EYA4 & NM_001301012 & $\begin{array}{l}\text { EYA transcriptional coactivator } \\
\text { and phosphatase } 4\end{array}$ & $\begin{array}{l}\text { RPII-704JI7.4, CMDIJ, } \\
\text { DFNAI0 }\end{array}$ & $\begin{array}{l}\text { Tyrosine phosphatase that } \\
\text { specifically dephosphorylates } \\
\text { "Tyr-142" of histone H2AX } \\
\text { (H2AXY I 42ph) }\end{array}$ & \\
\hline FAM47B & NM_|5263| & $\begin{array}{l}\text { Family with sequence similarity } \\
47 \text {, member B }\end{array}$ & RPI3-520K9.I & & \\
\hline FBXO34 & NM_0I7943 & F-box protein 34 & CGI-30I, Fbx34 & $\begin{array}{l}\text { Substrate-recognition component } \\
\text { of the SCF E3 ubiquitin ligase } \\
\text { complex }\end{array}$ & \\
\hline FKBPIO & NM_021939 & FK506 binding protein 10 & $\begin{array}{l}\text { PSEC0056, FKBP65, OIII, } \\
\text { OI6, PPIASE, hFKBP65 }\end{array}$ & $\begin{array}{l}\text { PPlases accelerate the folding of } \\
\text { proteins during protein synthesis }\end{array}$ & \\
\hline FKBP4 & NM_002014 & FK506 binding protein 4 & $\begin{array}{l}\text { FKBP5I, FKBP52, FKBP59, } \\
\mathrm{HBI}, \mathrm{Hsp} 56 \text {, PPlase, p52 }\end{array}$ & $\begin{array}{l}\text { May play a role in the intracellular } \\
\text { trafficking of heterooligomeric forms } \\
\text { of steroid hormone receptors }\end{array}$ & \\
\hline FKBP7 & NM_001I35212 & FK506 binding protein 7 & $\begin{array}{l}\text { UNQ670/PROI304, } \\
\text { FKBP23, PPlase }\end{array}$ & $\begin{array}{l}\text { PPlases accelerate the folding of } \\
\text { proteins during protein synthesis }\end{array}$ & \\
\hline FRAIOACI & NM_I45246 & $\begin{array}{l}\text { Fragile site, folic acid type, rare, } \\
\operatorname{fra}(10)(q 23.3) \text { or } \operatorname{fra}(10)(q 24.2) \\
\text { candidate I }\end{array}$ & $\begin{array}{l}\text { PRO2972, CI0orf4, } \\
\text { F26CII.I-like, FRAIOA }\end{array}$ & & \\
\hline FSIPI & NM_I52597 & Fibrous sheath interacting protein I & HSDIO & & \\
\hline FXYD6 & NM_00II6483I & $\begin{array}{l}\text { FXYD domain containing ion } \\
\text { transport regulator } 6\end{array}$ & UNQ52I/PROI056 & & \\
\hline GADD45G & NM_006705 & $\begin{array}{l}\text { Growth arrest and DNA-damage- } \\
\text { inducible, } \gamma\end{array}$ & $\begin{array}{l}\text { RPII-260L6.I, CR6, DDIT2, } \\
\text { GADD45 } \gamma \text {, GRPI7 }\end{array}$ & $\begin{array}{l}\text { Involved in the regulation of } \\
\text { growth and apoptosis }\end{array}$ & \\
\hline GATA6 & NM_005257 & GATA binding protein 6 & & $\begin{array}{l}\text { Regulates terminal differentiation } \\
\text { and/or proliferation }\end{array}$ & \\
\hline GCNTI & NM_001097633 & $\begin{array}{l}\text { Glucosaminyl ( } N \text {-acetyl) } \\
\text { transferase I, core } 2\end{array}$ & $\begin{array}{l}\text { RPII-2I4NI6.I, C2GNT, } \\
\text { C2GNT-L, C2GNTI, } \\
\text { G6NT, NACGT2, } \\
\text { NAGCT2 }\end{array}$ & $\begin{array}{l}\text { Forms critical branches in } \\
\text { O-glycans }\end{array}$ & \\
\hline GNA I3 & NM_00I 282425 & $\mathrm{G}$ protein, $\alpha 13$ & GI3 & $\begin{array}{l}\text { Modulators or transducers in } \\
\text { various transmembrane signaling } \\
\text { systems }\end{array}$ & \\
\hline
\end{tabular}


Table 5 (Continued)

\begin{tabular}{|c|c|c|c|c|c|}
\hline Gene symbol & $\begin{array}{l}\text { Accession } \\
\text { number }\end{array}$ & Full name & Alias & Function & $\begin{array}{l}\text { Cancer } \\
\text { gene }\end{array}$ \\
\hline GNBI & NM_00I 282538 & G protein, $\beta$ polypeptide I & RPI-283E3.7 & $\begin{array}{l}\text { A modulator or transducer in } \\
\text { various transmembrane signaling } \\
\text { systems }\end{array}$ & \\
\hline GPR/37B & NM_003272 & G protein-coupled receptor I37B & RP5-985LI9.I, TM7SFI & & \\
\hline GPR83 & NM_0I6540 & G protein-coupled receptor 83 & GIR, GPR72 & $\begin{array}{l}\text { Orphan receptor. Could be a } \\
\text { neuropeptide y receptor }\end{array}$ & \\
\hline GSTM2 & NM_000848 & $\begin{array}{l}\text { Glutathione S-transferase mu } 2 \\
\text { (muscle) }\end{array}$ & $\begin{array}{l}\text { GST4, GSTM-2, GTHMUS, } \\
\text { GSTM2 }\end{array}$ & $\begin{array}{l}\text { Conjugation of reduced gluta- } \\
\text { thione to a wide number of exoge- } \\
\text { nous and endogenous hydrophobic } \\
\text { electrophiles }\end{array}$ & \\
\hline HIFO & NM_005318 & $\mathrm{HI}$ histone family, member 0 & HIO, HIFV & $\begin{array}{l}\text { Histones } \mathrm{HI} \text { are necessary for } \\
\text { the condensation of nucleosome } \\
\text { chains into higher order structures }\end{array}$ & \\
\hline HERC3 & NM_00I27I602 & $\begin{array}{l}\text { HECT and RLD domain } \\
\text { containing E3 ubiquitin protein } \\
\text { ligase } 3\end{array}$ & & E3 ubiquitin-protein ligase & \\
\hline HEY2 & NM_0I2259 & $\begin{array}{l}\text { Hes-related family bHLH } \\
\text { transcription factor with YRPW } \\
\text { motif } 2\end{array}$ & $\begin{array}{l}\text { RPI-293L8.3, CHFI, } \\
\text { GRIDLOCK, GRL, HERPI, } \\
\text { HESR2, HRT2, bHLHb32 }\end{array}$ & $\begin{array}{l}\text { Downstream effector of Notch } \\
\text { signaling which may be required for } \\
\text { cardiovascular development }\end{array}$ & \\
\hline HIPK2 & NM_00III 3239 & $\begin{array}{l}\text { Homeodomain interacting } \\
\text { protein kinase } 2\end{array}$ & PRO0593 & $\begin{array}{l}\text { Protein kinase acting as } \\
\text { a corepressor of several } \\
\text { transcription factors }\end{array}$ & \\
\hline HMGB2 & NM_00II30688 & High mobility group box 2 & HMG2 & $\begin{array}{l}\text { Binds preferentially ssDNA and } \\
\text { unwinds double-stranded DNA }\end{array}$ & \\
\hline HNRPDL & NM_00I207000 & $\begin{array}{l}\text { Heterogeneous nuclear } \\
\text { ribonucleoprotein D-like }\end{array}$ & $\begin{array}{l}\text { HNRNP, HNRPDL, JKTBP, } \\
\text { JKTBP2, laAUFI }\end{array}$ & Acts as a transcriptional regulator & \\
\hline HOXAIO & NM_0I895I & Homeobox AlO & $\begin{array}{l}\text { HOXI, HOXI.8, HOXIH, } \\
\text { PL }\end{array}$ & $\begin{array}{l}\text { Sequence-specific transcription } \\
\text { factor }\end{array}$ & \\
\hline HOXAII & NM_005523 & Homeobox AlI & HOXI, HOXII & $\begin{array}{l}\text { Sequence-specific transcription } \\
\text { factor }\end{array}$ & Yes \\
\hline HSD I 7B3 & NM_000I97 & $\begin{array}{l}\text { Hydroxysteroid }(17-\beta) \\
\text { dehydrogenase } 3\end{array}$ & $\begin{array}{l}\text { RPII-240L7.3, EDHI7B3, } \\
\text { SDRI2C2 }\end{array}$ & $\begin{array}{l}\text { Favors the reduction of } \\
\text { androstenedione to testosterone }\end{array}$ & \\
\hline HSP9OBI & NM_003299 & $\begin{array}{l}\text { Heat-shock protein } 90 \mathrm{kDa} \beta \\
\text { (Grp94), member I }\end{array}$ & $\begin{array}{l}\text { ECGP, GP96, GRP94, HEL- } \\
\text { S- } 125 \mathrm{~m} \text {, HEL35, TRAI }\end{array}$ & $\begin{array}{l}\text { Molecular chaperone that } \\
\text { functions in the processing and } \\
\text { transport of secreted proteins }\end{array}$ & \\
\hline HSPAIB & NM_005346 & Heat-shock $70 \mathrm{kDa}$ protein IB & $\begin{array}{l}\text { DAAP-2 IF2.7, HSP70-IB, } \\
\text { HSP70-2 }\end{array}$ & $\begin{array}{l}\text { Stabilizes preexistent proteins } \\
\text { against aggregation and mediates } \\
\text { the folding of newly translated } \\
\text { polypeptides in the cytosol as well } \\
\text { as within organelles }\end{array}$ & \\
\hline ICMT & NM_0I2405 & $\begin{array}{l}\text { Isoprenylcysteine carboxyl } \\
\text { methyltransferase }\end{array}$ & $\begin{array}{l}\text { RPI-I 20G22.4, HSTEI4, } \\
\text { MST098, MSTP098, } \\
\text { PCCMT, PCMT, PPMT }\end{array}$ & $\begin{array}{l}\text { Catalyzes the posttranslational } \\
\text { methylation of isoprenylated } \\
\text { C-terminal cysteine } \\
\text { residues }\end{array}$ & \\
\hline IDS & NM_000202 & Iduronate 2-sulfatase & MPS2, SIDS & $\begin{array}{l}\text { Required for the lysosomal } \\
\text { degradation of heparan sulfate and } \\
\text { dermatan sulfate }\end{array}$ & \\
\hline INCENP & NM_00I040694 & $\begin{array}{l}\text { Inner centromere protein } \\
\text { antigens }\end{array}$ & & $\begin{array}{l}\text { Component of the chromosomal } \\
\text { passenger complex, a complex } \\
\text { that acts as a key regulator of } \\
\text { mitosis }\end{array}$ & \\
\hline IQCG & NM_00II34435 & IQ motif containing $G$ & CFAPI 22, DRC9 & & \\
\hline KAT2B & NM_003884 & $\mathrm{K}$ (lysine) acetyltransferase 2B & CAF, P/CAF, PCAF & $\begin{array}{l}\text { Functions as a HAT to promote } \\
\text { transcriptional activation }\end{array}$ & \\
\hline KBTBD3 & NM_I52433 & $\begin{array}{l}\text { Kelch repeat and BTB (POZ) } \\
\text { domain containing } 3\end{array}$ & BKLHD3 & & \\
\hline
\end{tabular}


Table 5 (Continued)

\begin{tabular}{|c|c|c|c|c|c|}
\hline Gene symbol & $\begin{array}{l}\text { Accession } \\
\text { number }\end{array}$ & Full name & Alias & Function & $\begin{array}{l}\text { Cancer } \\
\text { gene }\end{array}$ \\
\hline KBTBD7 & NM_032I38 & $\begin{array}{l}\text { Kelch repeat and BTB (POZ) } \\
\text { domain containing } 7\end{array}$ & & & \\
\hline KCTD3 & NM_016121 & $\begin{array}{l}\text { Potassium channel tetramerization } \\
\text { domain containing } 3\end{array}$ & RPII-5FI9.I, NY-REN-45 & & \\
\hline KIAAOIOI & NM_001029989 & KIAAOIOI & $\begin{array}{l}\text { L5, NS5ATP9, OEATC, } \\
\text { OEATC-I, OEATCI, PAF, } \\
\text { PAFI5, pI5(PAF), pI5/PAF, } \\
\text { PI5PAF }\end{array}$ & $\begin{array}{l}\text { May be involved in protection of } \\
\text { cells from UV-induced cell death }\end{array}$ & \\
\hline KIAA2026 & NM_0010I7969 & KIAA2026 & & & \\
\hline KLHLI 5 & NM_030624 & Kelch-like family member 15 & HEL-S-305 & $\begin{array}{l}\text { Probable substrate-specific adapter } \\
\text { of an E3 ubiquitin-protein ligase } \\
\text { complex which mediates the } \\
\text { ubiquitination and subsequent } \\
\text { proteasomal degradation of target } \\
\text { proteins }\end{array}$ & \\
\hline$K L R C 4$ & NM_01343I & $\begin{array}{l}\text { Killer cell lectin-like receptor } \\
\text { subfamily C, member } 4\end{array}$ & NKG2-F, NKG2F & $\begin{array}{l}\text { May play a role as a receptor for } \\
\text { the recognition of MHC class I } \\
\text { HLA-E molecules by NK cells }\end{array}$ & \\
\hline KRAS & NM_004985 & $\begin{array}{l}\text { Kirsten rat sarcoma viral } \\
\text { oncogene homolog }\end{array}$ & $\begin{array}{l}\text { C-K-RAS, CFC2, K-RAS2A, } \\
\text { K-RAS2B, K-RAS4A, } \\
\text { K-RAS4B, KI-RASI, KRAS2, } \\
\text { NS, NS3, RASK2, KRAS }\end{array}$ & $\begin{array}{l}\text { Binds GDP/GTP and possesses } \\
\text { intrinsic GTPase activity }\end{array}$ & Yes \\
\hline LFNG & NM_001040I67 & $\begin{array}{l}\text { LFNG O-fucosylpeptide } 3-\beta-N \text { - } \\
\text { acetylglucosaminyltransferase }\end{array}$ & SCDO3 & Glycosyltransferase & \\
\hline LPGATI & NM_0I4873 & $\begin{array}{l}\text { Lysophosphatidylglycerol } \\
\text { acyltransferase I }\end{array}$ & FAM34A, FAM34AI, NET8 & $\begin{array}{l}\text { Lysophoshatidylglycerol-specific } \\
\text { acyltransferase }\end{array}$ & \\
\hline$L R R C I 7$ & NM_001031692 & Leucine rich repeat containing 17 & $\begin{array}{l}\text { UNQ3076/PRO9909, } \\
\text { P37NB }\end{array}$ & $\begin{array}{l}\text { Involved in bone homeostasis, } \\
\text { acting as a negative regulator } \\
\text { of RANKL-induced osteoclast } \\
\text { precursor differentiation from } \\
\text { bone marrow precursors }\end{array}$ & \\
\hline LRRN3 & NM_001099658 & Leucine rich repeat neuronal 3 & $\begin{array}{l}\text { Nbla I0363, FIGLER5, } \\
\text { NLRR-3, NLRR3 }\end{array}$ & & \\
\hline LYSMD3 & NM_00I2868I2 & $\begin{array}{l}\text { LysM, putative peptidoglycan- } \\
\text { binding, domain containing } 3\end{array}$ & & & \\
\hline$M A P I B$ & NM_005909 & $\begin{array}{l}\text { Microtubule-associated } \\
\text { protein IB }\end{array}$ & FUTSCH, MAP5, PPPIRI02 & $\begin{array}{l}\text { May play a role in the cytoskeletal } \\
\text { changes that accompany neurite } \\
\text { extension }\end{array}$ & \\
\hline METAPI & NM_015I43 & Methionyl aminopeptidase I & MAPIA, MetAPIA & $\begin{array}{l}\text { Removes the amino-terminal } \\
\text { methionine from nascent proteins }\end{array}$ & \\
\hline MFAP3 & NM_00II35037 & Microfibrillar-associated protein 3 & & $\begin{array}{l}\text { Component of the elastin- } \\
\text { associated microfibrils }\end{array}$ & \\
\hline MIF & NM_0024I5 & $\begin{array}{l}\text { Macrophage migration inhibitory } \\
\text { factor (glycosylation-inhibiting } \\
\text { factor) }\end{array}$ & GIF, GLIF, MMIF & $\begin{array}{l}\text { The expression of MIF at sites } \\
\text { of inflammation suggests a role } \\
\text { for the mediator in regulating } \\
\text { the function of macrophage } \\
\text { in host defense. Also acts as a } \\
\text { phenylpyruvate tautomerase }\end{array}$ & \\
\hline MOB3B & NM_02476I & MOB kinase activator 3B & $\begin{array}{l}\text { C9orf35, MOBID, } \\
\text { MOBKL2B }\end{array}$ & May regulate the activity of kinases & \\
\hline MRPSI 4 & NM_022100 & $\begin{array}{l}\text { Mitochondrial ribosomal protein } \\
\text { SI4 }\end{array}$ & $\begin{array}{l}\text { DJ262DI2.2, HSMRPSI4, } \\
\text { MRP-SI4, SI } 4 \mathrm{mt}\end{array}$ & & \\
\hline$M T M R / 2$ & NM_001040446 & Myotubularin-related protein 12 & 3-PAP, PIP3AP & $\begin{array}{l}\text { Inactive phosphatase that plays a } \\
\text { role as an adapter for the phos- } \\
\text { phatase myotubularin to regulate } \\
\text { myotubularin intracellular location }\end{array}$ & \\
\hline
\end{tabular}


Table 5 (Continued)

\begin{tabular}{|c|c|c|c|c|c|}
\hline Gene symbol & $\begin{array}{l}\text { Accession } \\
\text { number }\end{array}$ & Full name & Alias & Function & $\begin{array}{l}\text { Cancer } \\
\text { gene }\end{array}$ \\
\hline MTRR & NM_002454 & $\begin{array}{l}\text { 5-Methyltetrahydrofolate- } \\
\text { homocysteine methyltransferase } \\
\text { reductase }\end{array}$ & MSR, cblE & $\begin{array}{l}\text { Involved in the reductive } \\
\text { regeneration of cob(I)alamin } \\
\text { cofactor required for the } \\
\text { maintenance of methionine } \\
\text { synthase in a functional state }\end{array}$ & \\
\hline MYO9A & NM_00690I & Myosin IXA & & $\begin{array}{l}\text { Myosins are actin-based motor } \\
\text { molecules with ATPase activity } \\
\text { Unconventional myosins serve in } \\
\text { intracellular movements }\end{array}$ & \\
\hline NCAPG & NM_022346 & $\begin{array}{l}\text { Non-SMC condensin I complex, } \\
\text { subunit G }\end{array}$ & $\begin{array}{l}\text { CAPG, CHCG, NY-MEL-3, } \\
\text { YCGI }\end{array}$ & $\begin{array}{l}\text { Regulatory subunit of the } \\
\text { condensin complex, a complex } \\
\text { required for conversion of } \\
\text { interphase chromatin into mitotic- } \\
\text { like condense chromosomes }\end{array}$ & \\
\hline$N K X 3-2$ & NM_00II89 & NK3 homeobox 2 & $\begin{array}{l}\text { BAPXI, NKX3.2, NKX3B, } \\
\text { SMMD }\end{array}$ & $\begin{array}{l}\text { Transcriptional repressor that } \\
\text { acts as a negative regulator of } \\
\text { chondrocyte maturation }\end{array}$ & \\
\hline$N L K$ & NM_01623I & Nemo-like kinase & & $\begin{array}{l}\text { Role in cell fate determination, } \\
\text { required for differentiation of bone } \\
\text { marrow stromal cells }\end{array}$ & \\
\hline NMRK2 & NM_001289117 & Nicotinamide riboside kinase 2 & ITGBIBP3, MIBP, NRK2 & & \\
\hline NOL4 & NM_00II 98546 & Nucleolar protein 4 & $\begin{array}{l}\text { HRIHFB2255, CTI25, } \\
\text { NOLP }\end{array}$ & & \\
\hline NUDTI 2 & NM_00I30074I & Nudix-type motif 12 & & $\begin{array}{l}\text { Hydrolyzes NAD }(\mathrm{P}) \mathrm{H} \text { to } \mathrm{NMNH} \\
\text { and AMP }\left(2^{\prime}, 5^{\prime}-\mathrm{ADP}\right) \text {, and } \\
\text { diadenosine diphosphate to AMP }\end{array}$ & \\
\hline$O A Z I$ & NM_00I30I020 & $\begin{array}{l}\text { Ornithine decarboxylase } \\
\text { antizyme I }\end{array}$ & AZI, OAZ & $\begin{array}{l}\text { Binds to and destabilizes ornithine } \\
\text { decarboxylase, which is then } \\
\text { degraded. Also inhibits cellular } \\
\text { uptake of polyamines by inactivating } \\
\text { the polyamine uptake transporter }\end{array}$ & \\
\hline $\mathrm{OFCCI}$ & NM_I53003 & Orofacial cleft I candidate I & MRDSI & & \\
\hline$O R I|A|$ & NM_0I3937 & $\begin{array}{l}\text { Olfactory receptor, family II, } \\
\text { subfamily A, member I }\end{array}$ & $\begin{array}{l}\text { DAAP-34II.2, 6MI-I8, } \\
\text { ORIIA2, dj994E9.6, } \\
\text { hs6MI-18 }\end{array}$ & Odorant receptor & \\
\hline OTUDI & NM_00I I 45373 & OTU deubiquitinase I & DUBA7, OTDCI & $\begin{array}{l}\text { Deubiquitinating enzyme that } \\
\text { specifically hydrolyzes 'Lys- } \\
63 \text { '-linked polyubiquitin to } \\
\text { monoubiquitin }\end{array}$ & \\
\hline OTX2 & NM_00I270523 & Orthodenticle homeobox 2 & CPHD6, MCOPS5 & $\begin{array}{l}\text { Probably plays a role in the develop- } \\
\text { ment of the brain and the sense } \\
\text { organs }\end{array}$ & \\
\hline PCAF & NM_003884 & $\mathrm{K}$ (lysine) acetyltransferase 2B & CAF, P/CAF, PCAF & $\begin{array}{l}\text { Functions as a component of the } \\
\text { PCAF complex }\end{array}$ & \\
\hline PCDHB8 & NM_019120 & Protocadherin $\beta 8$ & PCDH- $\beta 8$, PCDH3I & $\begin{array}{l}\text { Potential calcium-dependent cell- } \\
\text { adhesion protein }\end{array}$ & \\
\hline $\mathrm{PHOX} 2 \mathrm{~A}$ & NM_005I69 & Paired-like homeobox 2a & $\begin{array}{l}\text { ARIX, CFEOM2, FEOM2, } \\
\text { NCAM2, PMX } 2 A\end{array}$ & $\begin{array}{l}\text { May be involved in regulating the } \\
\text { specificity of expression of the } \\
\text { catecholamine biosynthetic genes }\end{array}$ & \\
\hline PIM3 & NM_00I00I852 & $\begin{array}{l}\text { Pim-3 proto-oncogene, serine/ } \\
\text { threonine kinase }\end{array}$ & CITF22-49E9.I, pim-3 & $\begin{array}{l}\text { May be involved in cell cycle } \\
\text { progression and antiapoptotic } \\
\text { process }\end{array}$ & \\
\hline PLA2G4C & NM_00II59322 & $\begin{array}{l}\text { Phospholipase A2, group IVC } \\
\text { (cytosolic, calcium-independent) }\end{array}$ & CPLA2- $\gamma$ & $\begin{array}{l}\text { Has a preference for arachidonic } \\
\text { acid at the sn- } 2 \text { position of } \\
\text { phosphatidylcholine as compared } \\
\text { with palmitic acid }\end{array}$ & \\
\hline
\end{tabular}


Table 5 (Continued)

\begin{tabular}{|c|c|c|c|c|c|}
\hline Gene symbol & $\begin{array}{l}\text { Accession } \\
\text { number }\end{array}$ & Full name & Alias & Function & $\begin{array}{l}\text { Cancer } \\
\text { gene }\end{array}$ \\
\hline PLAGI & NM_00III4634 & Pleiomorphic adenoma gene I & PSA, SGPA, ZNF9I2 & $\begin{array}{l}\text { Transcription factor whose } \\
\text { activation results in upregulation of } \\
\text { target genes, such as IGFIl, leading } \\
\text { to uncontrolled cell proliferation }\end{array}$ & Yes \\
\hline PLCL2 & NM_00II44382 & Phospholipase C-like 2 & PLCE2 & $\begin{array}{l}\text { May play a role in the regulation } \\
\text { of Ins }(1,4,5) \mathrm{P} 3 \text { around the } \\
\text { endoplasmic reticulum }\end{array}$ & \\
\hline$P L X D C 2$ & NM_00I282736 & Plexin domain containing 2 & $\begin{array}{l}\text { UNQ25 I4/PRO6003, } \\
\text { TEM7R }\end{array}$ & $\begin{array}{l}\text { May play a role in tumor } \\
\text { angiogenesis }\end{array}$ & \\
\hline PNPTI & NM_033109 & $\begin{array}{l}\text { Polyribonucleotide } \\
\text { nucleotidyltransferase I }\end{array}$ & $\begin{array}{l}\text { COXPDI3, DFNB70, } \\
\text { OLD35, PNPASE, old-35 }\end{array}$ & Involved in mRNA degradation & \\
\hline POLR2B & NM_000938 & $\begin{array}{l}\text { Polymerase (RNA) II (DNA } \\
\text { directed) polypeptide B }\end{array}$ & $\begin{array}{l}\text { POL2RB, RPB2, hRPBI40, } \\
\text { hsRPB2 }\end{array}$ & $\begin{array}{l}\text { DNA-dependent RNA polymerase } \\
\text { catalyzes the transcription of } \\
\text { DNA into RNA using the four } \\
\text { ribonucleoside triphosphates as } \\
\text { substrates }\end{array}$ & \\
\hline PPAI & NM_02II29 & Pyrophosphatase (inorganic) I & $\begin{array}{l}\text { RPI I-367H5.I, HEL-S-66p, } \\
\text { IOPPP, PP, PPI, SID6-806 I }\end{array}$ & & \\
\hline PPMIA & NM_021003 & $\begin{array}{l}\text { Protein phosphatase, } \mathrm{Mg}^{2+} / \mathrm{Mn}^{2+} \\
\text { dependent, IA }\end{array}$ & $\begin{array}{l}\text { PP2C-ALPHA, PP2CA, } \\
\text { PP2C } \alpha\end{array}$ & Enzyme with a broad specificity & \\
\hline PPP2CA & NM_0027I5 & $\begin{array}{l}\text { Protein phosphatase } 2 \text {, catalytic } \\
\text { subunit, } \alpha \text { isozyme }\end{array}$ & $\begin{array}{l}\text { PP2Ac, PP2CA, PP2C } \alpha \text {, } \\
\text { RP-C }\end{array}$ & $\begin{array}{l}\text { PP2A can modulate the activity } \\
\text { of phosphorylase B kinase casein } \\
\text { kinase } 2 \text {, mitogen-stimulated S6 } \\
\text { kinase, and MAP- } 2 \text { kinase }\end{array}$ & \\
\hline PPP2R5C & NM_00II6I725 & $\begin{array}{l}\text { Protein phosphatase } 2 \text {, regulatory } \\
\text { subunit } B^{\prime}, \gamma\end{array}$ & B56G, PR6IG & $\begin{array}{l}\text { The } B \text { regulatory subunit might } \\
\text { modulate substrate selectivity } \\
\text { and catalytic activity, and also } \\
\text { might direct the localization of the } \\
\text { catalytic enzyme to a particular } \\
\text { subcellular compartment }\end{array}$ & \\
\hline PRDX3 & NM_006793 & Peroxiredoxin 3 & $\begin{array}{l}\text { AOP-I, AOPI, HBCI89, } \\
\text { MER5, PROI748, SP-22, } \\
\text { prx-III }\end{array}$ & $\begin{array}{l}\text { Involved in redox regulation of } \\
\text { the cell }\end{array}$ & \\
\hline PRLR & NM_000949 & Prolactin receptor & HPRL, MFAB, hPRLrl & $\begin{array}{l}\text { This is a receptor for the anterior } \\
\text { pituitary hormone prolactin }\end{array}$ & \\
\hline PROSC & NM_007198 & $\begin{array}{l}\text { Proline synthetase co-transcribed } \\
\text { homolog (bacterial) }\end{array}$ & & & \\
\hline PROXI & NM_00I2706I6 & Prospero homeobox I & & $\begin{array}{l}\text { May play a fundamental role in early } \\
\text { development of central nervous } \\
\text { system }\end{array}$ & \\
\hline PRR4 & NM_001098538 & Proline rich 4 (lacrimal) & LPRP, PROL4 & & \\
\hline PTGS2 & NM_000963 & $\begin{array}{l}\text { Prostaglandin-endoperoxide } \\
\text { synthase } 2 \text { (prostaglandin G/H } \\
\text { synthase and cyclooxygenase) }\end{array}$ & $\begin{array}{l}\text { COX-2, COX2, GRIPGHS, } \\
\text { PGG/HS, PGHS-2, PHS-2, } \\
\text { hCox-2 }\end{array}$ & $\begin{array}{l}\text { May have a role as a major } \\
\text { mediator of inflammation and/or } \\
\text { a role for prostanoid signaling in } \\
\text { activity-dependent plasticity }\end{array}$ & \\
\hline PTPLAD / & NM_016395 & $\begin{array}{l}\text { Protein tyrosine phosphatase-like } \\
\text { A domain containing I }\end{array}$ & B-INDI, HACD3, HSPCI2I & $\begin{array}{l}\text { Involved in Racl-signaling pathways } \\
\text { leading to the modulation of gene } \\
\text { expression }\end{array}$ & \\
\hline PTPRZI & NM_00I206838 & $\begin{array}{l}\text { Protein tyrosine phosphatase, } \\
\text { receptor-type, Z polypeptide I }\end{array}$ & 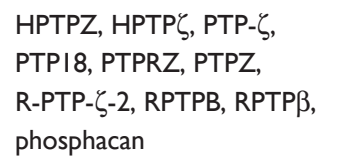 & $\begin{array}{l}\text { May be involved in the regulation } \\
\text { of specific developmental processes } \\
\text { in the central nervous system }\end{array}$ & \\
\hline$R A B 8 B$ & NM_0I6530 & $\begin{array}{l}\text { RAB8B, member RAS oncogene } \\
\text { family }\end{array}$ & & $\begin{array}{l}\text { May be involved in vesicular } \\
\text { trafficking and neurotransmitter } \\
\text { release }\end{array}$ & \\
\hline RASSF6 & NM_00I27039I & $\begin{array}{l}\text { Ras association (RalGDS/AF-6) } \\
\text { domain family member } 6\end{array}$ & & May act as a Ras effector protein & \\
\hline
\end{tabular}


Table 5 (Continued)

\begin{tabular}{|c|c|c|c|c|c|}
\hline Gene symbol & $\begin{array}{l}\text { Accession } \\
\text { number }\end{array}$ & Full name & Alias & Function & $\begin{array}{l}\text { Cancer } \\
\text { gene }\end{array}$ \\
\hline RBM $/ 5$ & NM_00I20I545 & RNA binding motif protein I5 & OTT, OTTI, SPEN & $\begin{array}{l}\text { May be implicated in HOX gene } \\
\text { regulation }\end{array}$ & \\
\hline$R L F$ & NM_0I242I & Rearranged L-myc fusion & $\begin{array}{l}\text { RPI-39G22.I, ZN-15L, } \\
\text { ZNF292L }\end{array}$ & $\begin{array}{l}\text { May be involved in transcriptional } \\
\text { regulation }\end{array}$ & \\
\hline ROPNIL & NM_00I20I466 & $\begin{array}{l}\text { Rhophilin associated tail protein } \\
\text { I-like }\end{array}$ & RPII-ICI.7, ASP, RSPHII & & \\
\hline RPSI 4 & NM_00I025070 & Ribosomal protein SI4 & PRO2640, EMTB, SI4 & & \\
\hline $\begin{array}{l}\text { RTELI- } \\
\text { TNFRSF6B }\end{array}$ & NR_037882 & $\begin{array}{l}\text { RTELI-TNFRSF6B readthrough } \\
\text { (NMD candidate) }\end{array}$ & & & \\
\hline SIOOAI & NM_00627I & SI00 calcium binding protein AI & $\begin{array}{l}\text { RPI-I78FI5.I, SI00, } \\
\text { SI00- } \alpha, \text { SI00A }\end{array}$ & $\begin{array}{l}\text { Weakly binds calcium but binds } \\
\text { zinc very tightly-distinct binding } \\
\text { sites with different affinities exist } \\
\text { for both ions on each monomer }\end{array}$ & \\
\hline SCAMP2 & NM_005697 & $\begin{array}{l}\text { Secretory carrier membrane } \\
\text { protein } 2\end{array}$ & & $\begin{array}{l}\text { Functions in post-Golgi recycling } \\
\text { pathways. Acts as a recycling } \\
\text { carrier to the cell surface }\end{array}$ & \\
\hline SEPT2 & NM_00I00849I & Septin 2 & $\begin{array}{l}\text { DIFF6, NEDD-5, NEDD5, } \\
\text { Pnutl3, hNedd5 }\end{array}$ & $\begin{array}{l}\text { Required for normal progress } \\
\text { through mitosis. Involved in } \\
\text { cytokinesis }\end{array}$ & \\
\hline SF3B3 & NM_0I2426 & Splicing factor $3 \mathrm{~b}$, subunit 3 & $\begin{array}{l}\text { RSEI, SAPI } 30, \text { SF3bI30, } \\
\text { STAFI3 }\end{array}$ & $\begin{array}{l}\text { Subunit of the splicing factor SF3B } \\
\text { required for 'A' complex assembly } \\
\text { formed by the stable binding of } \\
\text { U2 snRNP to the branch point } \\
\text { sequence in pre-mRNA }\end{array}$ & \\
\hline SH3BGRL & NM_003022 & $\begin{array}{l}\text { SH3 domain binding glutamate- } \\
\text { rich protein like }\end{array}$ & HEL-S-II5, SH3BGR & & \\
\hline SIX6 & NM_007374 & SIX homeobox 6 & МСOРCT2, OPTX2, Six9 & $\begin{array}{l}\text { May be involved in eye } \\
\text { development }\end{array}$ & \\
\hline SLC37A3 & NM_00I 287498 & $\begin{array}{l}\text { Solute carrier family } 37 \text {, } \\
\text { member } 3\end{array}$ & & & \\
\hline SLC7AII & NM_0I433I & $\begin{array}{l}\text { Solute carrier family } 7 \text { (anionic } \\
\text { amino acid transporter light } \\
\text { chain, xc-system), member II }\end{array}$ & CCBRI, xCT & $\begin{array}{l}\text { Sodium-independent, high-affinity } \\
\text { exchange of anionic amino acids } \\
\text { with high specificity for anionic } \\
\text { form of cystine and glutamate }\end{array}$ & \\
\hline SLCO2AI & NM_005630 & $\begin{array}{l}\text { Solute carrier organic anion } \\
\text { transporter family, member } 2 \mathrm{Al}\end{array}$ & $\begin{array}{l}\text { MATRI, OATP2AI, PGT, } \\
\text { PHOAR2, SLC2IA2 }\end{array}$ & $\begin{array}{l}\text { May mediate the release of newly } \\
\text { synthesized prostaglandins from } \\
\text { cells, the transepithelial transport of } \\
\text { prostaglandins, and the clearance of } \\
\text { prostaglandins from the circulation }\end{array}$ & \\
\hline SMAD5 & NM_001001419 & SMAD family member 5 & DWFC, JV5-I, MADH5 & $\begin{array}{l}\text { Transcriptional modulator activated } \\
\text { by BMP type I receptor kinase }\end{array}$ & \\
\hline SMCHDI & NM_0I5295 & $\begin{array}{l}\text { Structural maintenance of } \\
\text { chromosomes flexible hinge } \\
\text { domain containing I }\end{array}$ & & $\begin{array}{l}\text { Required for maintenance of } \\
X \text { inactivation in females and } \\
\text { hypermethylation of } C_{p} G \text { islands } \\
\text { associated with inactive } X\end{array}$ & \\
\hline SNAI2 & NM_003068 & Snail family zinc finger 2 & $\begin{array}{l}\text { SLUG, SLUGHI, SNAIL2, } \\
\text { WS2D }\end{array}$ & $\begin{array}{l}\text { Transcriptional repressor. Involved } \\
\text { in the generation and migration of } \\
\text { neural crest cells }\end{array}$ & \\
\hline SRPK2 & NM_00I278273 & SRSF protein kinase 2 & SFRSK2 & $\begin{array}{l}\text { Phosphorylates RS domain- } \\
\text { containing proteins }\end{array}$ & \\
\hline TAAR6 & NM_I75067 & $\begin{array}{l}\text { Trace amine associated } \\
\text { receptor } 6\end{array}$ & $\begin{array}{l}\text { RPII-295F4.3, TA4, TAR4, } \\
\text { TAR6, TRAR4, taR-4, taR-6 }\end{array}$ & $\begin{array}{l}\text { Orphan receptor. Could be a } \\
\text { receptor for trace amines }\end{array}$ & \\
\hline TAFI5 & NM_003487 & $\begin{array}{l}\text { TAFI } 5 \text { RNA polymerase II, TBP- } \\
\text { associated factor }\end{array}$ & $\begin{array}{l}\text { Npl3, RBP56, TAF2N, } \\
\text { TAFII68 }\end{array}$ & $\begin{array}{l}\text { RNA and ssDNA-binding protein } \\
\text { that may play specific roles during } \\
\text { transcription initiation at distinct } \\
\text { promoters }\end{array}$ & Yes \\
\hline
\end{tabular}


Table 5 (Continued)

\begin{tabular}{|c|c|c|c|c|c|}
\hline Gene symbol & $\begin{array}{l}\text { Accession } \\
\text { number }\end{array}$ & Full name & Alias & Function & $\begin{array}{l}\text { Cancer } \\
\text { gene }\end{array}$ \\
\hline TAF2 & NM_003184 & $\begin{array}{l}\text { TAF2 RNA polymerase II, TBP- } \\
\text { associated factor }\end{array}$ & $\begin{array}{l}\text { CIFI50, MRT40B, TAFIII50, } \\
\text { TAF2 }\end{array}$ & $\begin{array}{l}\text { Transcription factor TFIID is one } \\
\text { of the general factors required for } \\
\text { accurate and regulated initiation by } \\
\text { RNA polymerase II }\end{array}$ & \\
\hline TAF6L & NM_006473 & $\begin{array}{l}\text { TAF6-like RNA PCAF-associated } \\
\text { factor }\end{array}$ & PAF65A & $\begin{array}{l}\text { Functions as a component of the } \\
\text { PCAF complex }\end{array}$ & \\
\hline$T B X 4$ & NM_018488 & T-box 4 & SPS & $\begin{array}{l}\text { Involved in the transcriptional } \\
\text { regulation of genes required for } \\
\text { mesoderm differentiation }\end{array}$ & \\
\hline TCF2I & NM_003206 & Transcription factor 21 & PODI, bHLHa23 & $\begin{array}{l}\text { Involved in epithelial-mesenchymal } \\
\text { interactions in kidney and lung } \\
\text { morphogenesis that include } \\
\text { epithelial differentiation and } \\
\text { branching morphogenesis }\end{array}$ & \\
\hline THUMPD I & NM_0I7736 & THUMP domain containing I & & & \\
\hline TM9SF3 & NM_020I23 & $\begin{array}{l}\text { Transmembrane } 9 \text { superfamily } \\
\text { member } 3\end{array}$ & $\begin{array}{l}\text { RPI I-34E5. I, EP70-P-iso, } \\
\text { SMBP }\end{array}$ & & \\
\hline TMEM / 4A & NM_0I405I & Transmembrane protein I4A & PTDOII, C6orf73 & & \\
\hline TMEM45A & NM_0I8004 & Transmembrane protein $45 \mathrm{~A}$ & DERP7 & & \\
\hline TMPO & NM_001032283 & Thymopoietin & $\begin{array}{l}\text { CMDIT, LAP2, LEMD4, } \\
\text { PRO0868, TP }\end{array}$ & $\begin{array}{l}\text { May help direct the assembly of the } \\
\text { nuclear lamina and thereby help } \\
\text { maintain the structural organization } \\
\text { of the nuclear envelope }\end{array}$ & \\
\hline TMPRSSI IA & NM_00III4387 & $\begin{array}{l}\text { Transmembrane protease, serine } \\
\text { IIA }\end{array}$ & ECRGI & $\begin{array}{l}\text { Probable serine protease, which may } \\
\text { play a role in cellular senescence }\end{array}$ & \\
\hline TNPOI & NM_002270 & Transportin I & $\begin{array}{l}\text { IPO2, KPNB2, MIP, MIPI, } \\
\text { TRN }\end{array}$ & $\begin{array}{l}\text { Functions in nuclear protein import } \\
\text { as nuclear transport receptor }\end{array}$ & \\
\hline TNRC6C & NM_00II42640 & $\begin{array}{l}\text { Trinucleotide repeat containing } \\
6 \mathrm{C}\end{array}$ & & $\begin{array}{l}\text { Plays a role in RNA-mediated gene } \\
\text { silencing by micro-RNAs }\end{array}$ & \\
\hline TRUBI & NM_139169 & $\begin{array}{l}\text { TruB pseudouridine (psi) } \\
\text { synthase family member I }\end{array}$ & PUS4 & $\begin{array}{l}\text { May be responsible for synthesis of } \\
\text { psi from uracil in transfer RNAs }\end{array}$ & \\
\hline TSGIOI & NM_006292 & Tumor susceptibility I0I & TSGI0, VPS23 & $\begin{array}{l}\text { Component of the ESCRT-I } \\
\text { complex, a regulator of vesicular } \\
\text { trafficking process }\end{array}$ & \\
\hline TSHR & NM_000369 & $\begin{array}{l}\text { Thyroid stimulating hormone } \\
\text { receptor }\end{array}$ & CHNGI, LGR3, hTSHR-I & $\begin{array}{l}\text { Receptor for thyrothropin. Plays a } \\
\text { central role in controlling thyroid } \\
\text { cell metabolism }\end{array}$ & Yes \\
\hline TUSCI & NM_001004I25 & Tumor suppressor candidate I & TSG-9, TSG9 & & \\
\hline TWFI & NM_001242397 & Twinfilin actin-binding protein I & A6, PTK9 & $\begin{array}{l}\text { Actin-binding protein involved in } \\
\text { motile and morphological processes }\end{array}$ & \\
\hline UGT3AI & NM_001171873 & $\begin{array}{l}\text { UDP glycosyltransferase } 3 \text { family, } \\
\text { polypeptide AI }\end{array}$ & & $\begin{array}{l}\text { UDP-glucuronosyltransferases } \\
\text { catalyze phase Il biotransformation } \\
\text { reactions }\end{array}$ & \\
\hline USP28 & NM_001301029 & Ubiquitin specific peptidase 28 & & $\begin{array}{l}\text { Deubiquitinase involved in DNA } \\
\text { damage response checkpoint and } \\
\text { MYC proto-oncogene stability }\end{array}$ & \\
\hline VBPI & NM_003372 & $\begin{array}{l}\text { Von Hippel-Lindau binding } \\
\text { protein I }\end{array}$ & $\begin{array}{l}\text { RPI3-228JI3.4, PFD3, } \\
\text { PFDN3, VBP-I }\end{array}$ & $\begin{array}{l}\text { Binds specifically to c-CPN and } \\
\text { transfers target proteins to it }\end{array}$ & \\
\hline WDR33 & NM_001006622 & WD repeat domain 33 & NETI4, WDCI46 & $\begin{array}{l}\text { Essential for both cleavage and poly- } \\
\text { adenylation of pre-mRNA } 3{ }^{\prime} \text { ends }\end{array}$ & \\
\hline WNTI 6 & NM_0I6087 & $\begin{array}{l}\text { Wingless-type MMTV integration } \\
\text { site family, member } 16\end{array}$ & & $\begin{array}{l}\text { Ligand for members of the Frizzled } \\
\text { family of seven transmembrane } \\
\text { receptors }\end{array}$ & \\
\hline WNT2 & NM_00339I & $\begin{array}{l}\text { Wingless-type MMTV integration } \\
\text { site family member } 2\end{array}$ & INTILI, IRP & $\begin{array}{l}\text { Ligand for members of the Frizzled } \\
\text { family of seven transmembrane } \\
\text { receptors }\end{array}$ & \\
\hline
\end{tabular}


Table 5 (Continued)

\begin{tabular}{|c|c|c|c|c|c|}
\hline Gene symbol & $\begin{array}{l}\text { Accession } \\
\text { number }\end{array}$ & Full name & Alias & Function & $\begin{array}{l}\text { Cancer } \\
\text { gene }\end{array}$ \\
\hline WNT3A & NM_033|3I & $\begin{array}{l}\text { Wingless-type MMTV integration } \\
\text { site family, member } 3 \mathrm{~A}\end{array}$ & & $\begin{array}{l}\text { Ligand for members of the Frizzled } \\
\text { family of seven transmembrane } \\
\text { receptors }\end{array}$ & \\
\hline YYI & NM_003403 & YYI transcription factor & $\begin{array}{l}\text { DELTA, INO80S, NF-EI, } \\
\text { UCRBP, YIN-YANG-I }\end{array}$ & $\begin{array}{l}\text { May play an important role in } \\
\text { development and differentiation }\end{array}$ & \\
\hline ZIC2 & NM_007I29 & Zinc family member 2 & HPE5 & Involved in cerebellar development & \\
\hline ZNFI2 & NM_006956 & Zinc finger protein 12 & $\begin{array}{l}\text { GIOT-3, HZFII, KOX3, } \\
\text { ZNF325 }\end{array}$ & $\begin{array}{l}\text { May be involved in transcriptional } \\
\text { regulation }\end{array}$ & \\
\hline ZNFI2I & NM_00I008727 & Zinc finger protein 121 & DI9S204, ZHC32, ZNF20 & $\begin{array}{l}\text { May be involved in transcriptional } \\
\text { regulation }\end{array}$ & \\
\hline ZNFI32 & NM_003433 & Zinc finger protein 132 & $\mathrm{pHZ}-12$ & $\begin{array}{l}\text { May be involved in transcriptional } \\
\text { regulation }\end{array}$ & \\
\hline ZNFI80 & NM_00I278508 & Zinc finger protein 180 & $\mathrm{HHZI} 68$ & $\begin{array}{l}\text { May be involved in transcriptional } \\
\text { regulation }\end{array}$ & \\
\hline ZNF238 & NM_00I278I96 & $\begin{array}{l}\text { Zinc finger and BTB domain } \\
\text { containing } 18\end{array}$ & $\begin{array}{l}\text { C2H2-I7I, MRD22, RP58, } \\
\text { TAZ-I, ZNFI8 }\end{array}$ & $\begin{array}{l}\text { Sequence-specific DNA-binding } \\
\text { protein with transcriptional } \\
\text { repression activity }\end{array}$ & \\
\hline ZNF25 & NM_I450II & Zinc finger protein 25 & KOXI9, Zfp9 & $\begin{array}{l}\text { May be involved in transcriptional } \\
\text { regulation }\end{array}$ & \\
\hline ZNF30 & NM_00I099437 & Zinc finger protein 30 & KOX28 & $\begin{array}{l}\text { May be involved in transcriptional } \\
\text { regulation }\end{array}$ & \\
\hline ZNF426 & NM_00I300883 & Zinc finger protein 426 & & $\begin{array}{l}\text { May be involved in transcriptional } \\
\text { regulation }\end{array}$ & \\
\hline ZNF558 & NM_I44693 & Zinc finger protein 558 & & $\begin{array}{l}\text { May be involved in transcriptional } \\
\text { regulation }\end{array}$ & \\
\hline ZNF562 & NM_00II3003I & Zinc finger protein 562 & & $\begin{array}{l}\text { May be involved in transcriptional } \\
\text { regulation }\end{array}$ & \\
\hline ZNF564 & NM_I44976 & Zinc finger protein 564 & & $\begin{array}{l}\text { May be involved in transcriptional } \\
\text { regulation }\end{array}$ & \\
\hline ZNF594 & NM_032530 & Zinc finger protein 594 & hCG_I 775942 & $\begin{array}{l}\text { May be involved in transcriptional } \\
\text { regulation }\end{array}$ & \\
\hline ZNF644 & NM_0I6620 & Zinc finger protein 644 & $\begin{array}{l}\text { BM-005, MYP2I, NatF, } \\
\text { ZEP-2 }\end{array}$ & $\begin{array}{l}\text { May be involved in transcriptional } \\
\text { regulation }\end{array}$ & \\
\hline ZNF652 & NM_00II45365 & Zinc finger protein 652 & & $\begin{array}{l}\text { Functions as a transcriptional } \\
\text { repressor }\end{array}$ & \\
\hline ZNF700 & NM_00127I848 & Zinc finger protein 700 & & $\begin{array}{l}\text { May be involved in transcriptional } \\
\text { regulation }\end{array}$ & \\
\hline ZNF703 & NM_025069 & Zinc finger protein 703 & ZEPPOI, ZNF503L, ZPOI & $\begin{array}{l}\text { May function as a transcriptional } \\
\text { repressor }\end{array}$ & \\
\hline ZNF7III & NM_021998 & Zinc finger protein 711 & $\begin{array}{l}\text { CMPXI, MRX97, ZNF4, ZNF5, } \\
\text { ZNF6, Zfp7II, dJ75NI3.I }\end{array}$ & $\begin{array}{l}\text { May be involved in transcriptional } \\
\text { regulation }\end{array}$ & \\
\hline ZNF763 & NM_00I0I2753 & Zinc finger protein 763 & ZNF, ZNF440L & $\begin{array}{l}\text { May be involved in transcriptional } \\
\text { regulation }\end{array}$ & \\
\hline ZNF780A & NM_00I0I0880 & Zinc finger protein $780 \mathrm{~A}$ & ZNF780 & $\begin{array}{l}\text { May be involved in transcriptional } \\
\text { regulation }\end{array}$ & \\
\hline
\end{tabular}

Abbreviations: CLL, chronic lymphocytic leukemia; HLA, human leukocyte antigen; HSP, heat shock protein; IGF, insulin-like growth factor; IL, interleukin; mRNA, messenger RNA; NK cells, natural killer cells; ssDNA, single-stranded DNA; UV, ultraviolet.

CCDC6, CCND1, CDX2, EP300, HOXA11, KRAS, PLAG1, TAF15, and TSHR.

As shown in Table 6, our DAVID analysis showed that there were 16 functional clusters that were identified to be enriched with an enrichment score $>1.0$ in the target list of hsa-miR-181a-5p, based on TarBase. The functions of these clusters involved negative regulation of macromolecule biosynthetic process, negative regulation of the cellular biosynthetic process, negative regulation of biosynthetic process, negative regulation of apoptosis, negative regulation of programmed cell death, negative regulation of cell death, negative regulation of transcription, negative regulation of nucleobase, nucleoside, nucleotide, and nucleic acid metabolic processes, negative regulation of nitrogen compound metabolic process, 
Table 6 The top enriched clusters (enrich score $>$ I) by DAVID for the targets of hsa-miR-I8Ia-5p from TarBase 6.0

\begin{tabular}{|c|c|c|c|c|}
\hline Category & Term & Gene count & $P$-value & FDR \\
\hline Annotation cluster I & Enrichment score: 4.3 & & & \\
\hline INTERPRO & Zinc finger, $\mathrm{C} 2 \mathrm{H} 2$-type & 24 & $2.20 \mathrm{E}-05$ & 4.60E-03 \\
\hline INTERPRO & Zinc finger, $\mathrm{C} 2 \mathrm{H} 2$-like & 24 & $2.90 \mathrm{E}-05$ & $3.90 \mathrm{E}-03$ \\
\hline SMART & Zinc finger_C2H2 & 24 & $2.00 \mathrm{E}-04$ & I.90E-02 \\
\hline Annotation cluster 2 & Enrichment score: 3.04 & & & \\
\hline GOTERM_BP_FAT & Negative regulation of macromolecule biosynthetic process & 17 & 7.10E-04 & $9.80 \mathrm{E}-02$ \\
\hline GOTERM_BP_FAT & Negative regulation of cellular biosynthetic process & 17 & $9.30 \mathrm{E}-04$ & I.20E-0। \\
\hline GOTERM_BP_FAT & Negative regulation of biosynthetic process & 17 & I.20E-03 & $9.50 \mathrm{E}-02$ \\
\hline Annotation cluster 3 & Enrichment score: 2.97 & & & \\
\hline GOTERM_BP_FAT & Negative regulation of apoptosis & 13 & $9.70 \mathrm{E}-04$ & I.IOE-0I \\
\hline GOTERM_BP_FAT & Negative regulation of programmed cell death & 13 & I.IOE-03 & I.00E-0 I \\
\hline GOTERM_BP_FAT & Negative regulation of cell death & 13 & I. I0E-03 & $9.70 \mathrm{E}-02$ \\
\hline Annotation cluster 4 & Enrichment score: 2.67 & & & \\
\hline GOTERM_BP_FAT & Negative regulation of transcription & 15 & I.00E-03 & I.00E-0I \\
\hline GOTERM_BP_FAT & $\begin{array}{l}\text { Negative regulation of nucleobase, nucleoside, nucleotide } \\
\text { and nucleic acid metabolic process }\end{array}$ & 15 & $2.90 \mathrm{E}-03$ & I.50E-0I \\
\hline GOTERM_BP_FAT & Negative regulation of nitrogen compound metabolic process & 15 & $3.20 \mathrm{E}-03$ & I.50E-0। \\
\hline Annotation cluster 5 & Enrichment score: 2.14 & & & \\
\hline GOTERM_BP_FAT & Lung development & 6 & $6.20 \mathrm{E}-03$ & $2.30 \mathrm{E}-0 \mathrm{I}$ \\
\hline GOTERM_BP_FAT & Respiratory tube development & 6 & 7.00E-03 & 2.40E-0I \\
\hline GOTERM_BP_FAT & Respiratory system development & 6 & $8.90 \mathrm{E}-03$ & $2.50 \mathrm{E}-0 \mathrm{I}$ \\
\hline Annotation cluster 6 & Enrichment score: 1.75 & & & \\
\hline GOTERM_BP_FAT & Positive regulation of protein polymerization & 4 & $3.00 \mathrm{E}-03$ & I.50E-0| \\
\hline GOTERM_BP_FAT & Positive regulation of protein complex assembly & 4 & $7.90 \mathrm{E}-03$ & $2.60 \mathrm{E}-0 \mathrm{I}$ \\
\hline GOTERM_BP_FAT & Regulation of protein polymerization & 4 & 4.60E-02 & 4.90E-0I \\
\hline GOTERM_BP_FAT & Regulation of protein complex assembly & 4 & $9.00 \mathrm{E}-02$ & $6.00 \mathrm{E}-0 \mathrm{I}$ \\
\hline Annotation cluster 7 & Enrichment score: 1.59 & & & \\
\hline GOTERM_BP_FAT & Regulation of phosphorylation & 12 & $2.20 \mathrm{E}-02$ & $3.50 \mathrm{E}-0 \mathrm{I}$ \\
\hline GOTERM_BP_FAT & Regulation of phosphate metabolic process & 12 & $2.80 \mathrm{E}-02$ & $3.90 \mathrm{E}-0 \mathrm{I}$ \\
\hline GOTERM_BP_FAT & Regulation of phosphorus metabolic process & 12 & $2.80 \mathrm{E}-02$ & $3.90 \mathrm{E}-0 \mathrm{I}$ \\
\hline Annotation cluster 8 & Enrichment score: 1.57 & & & \\
\hline INTERPRO & Secreted growth factor Wnt protein, conserved site & 3 & $1.90 \mathrm{E}-02$ & $7.90 \mathrm{E}-0 \mathrm{I}$ \\
\hline INTERPRO & Secreted growth factor Wnt protein & 3 & $1.90 \mathrm{E}-02$ & $7.90 \mathrm{E}-0 \mathrm{I}$ \\
\hline INTERPRO & Wnt superfamily & 3 & I.90E-02 & $7.90 \mathrm{E}-0 \mathrm{I}$ \\
\hline PIR_SUPERFAMILY & PIRSF00 I784:int-I transforming protein & 3 & $2.00 \mathrm{E}-02$ & $8.60 \mathrm{E}-0 \mathrm{I}$ \\
\hline SMART & Wntl & 3 & $2.50 \mathrm{E}-02$ & $5.50 \mathrm{E}-0 \mathrm{I}$ \\
\hline GOTERM_BP_FAT & Wnt receptor signaling pathway, calcium modulating pathway & 3 & $2.50 \mathrm{E}-02$ & 3.70E-0I \\
\hline KEGG_PATHWAY & Basal cell carcinoma & 3 & I.40E-0| & 7.00E-0I \\
\hline Annotation cluster 9 & Enrichment score: 1.56 & & & \\
\hline GOTERM_BP_FAT & Positive regulation of microtubule polymerization & 3 & $8.40 \mathrm{E}-03$ & $2.50 \mathrm{E}-0 \mathrm{I}$ \\
\hline GOTERM_BP_FAT & Regulation of microtubule polymerization & 3 & $9.80 \mathrm{E}-03$ & $2.60 \mathrm{E}-0 \mathrm{I}$ \\
\hline GOTERM_BP_FAT & $\begin{array}{l}\text { Positive regulation of microtubule polymerization or } \\
\text { depolymerization }\end{array}$ & 3 & $9.80 \mathrm{E}-03$ & 2.60E-0I \\
\hline GOTERM_BP_FAT & Regulation of microtubule polymerization or depolymerization & 3 & 5. I0E-02 & 5.IOE-0I \\
\hline GOTERM_BP_FAT & Regulation of microtubule cytoskeleton organization & 3 & $8.70 \mathrm{E}-02$ & $6.00 \mathrm{E}-0 \mathrm{I}$ \\
\hline GOTERM_BP_FAT & Regulation of microtubule-based process & 3 & I.IOE-0I & $6.60 \mathrm{E}-0 \mathrm{I}$ \\
\hline Annotation cluster 10 & Enrichment score: 1.49 & & & \\
\hline GOTERM_BP_FAT & Positive regulation of cytoskeleton organization & 5 & I.90E-03 & I.20E-0I \\
\hline GOTERM_BP_FAT & Positive regulation of organelle organization & 5 & I.60E-02 & $3.30 \mathrm{E}-0 \mathrm{I}$ \\
\hline GOTERM_BP_FAT & Regulation of cytoskeleton organization & 5 & $7.60 \mathrm{E}-02$ & $5.80 \mathrm{E}-0 \mathrm{I}$ \\
\hline GOTERM_BP_FAT & Regulation of cellular component biogenesis & 5 & $8.60 \mathrm{E}-02$ & $6.00 \mathrm{E}-0 \mathrm{I}$ \\
\hline GOTERM_BP_FAT & Positive regulation of cellular component organization & 5 & I.60E-0| & 7.30E-0I \\
\hline Annotation cluster I I & Enrichment score: 1.36 & & & \\
\hline UP_SEQ_FEATURE & DNA-binding region: Homeobox & 7 & I.60E-02 & 4.70E-0I \\
\hline INTERPRO & Homeobox, conserved site & 7 & $4.60 \mathrm{E}-02$ & $9.10 \mathrm{E}-01$ \\
\hline INTERPRO & Homeobox & 7 & 4.90E-02 & $9.00 \mathrm{E}-01$ \\
\hline INTERPRO & Homeodomain-related & 7 & 5. $10 \mathrm{E}-02$ & $8.60 \mathrm{E}-0 \mathrm{I}$ \\
\hline SMART & Hox & 7 & $8.50 \mathrm{E}-02$ & $7.60 \mathrm{E}-0 \mathrm{I}$ \\
\hline
\end{tabular}


Table 6 (Continued)

\begin{tabular}{|c|c|c|c|c|}
\hline Category & Term & Gene count & $P$-value & FDR \\
\hline Annotation cluster 12 & Enrichment score: I.35 & & & \\
\hline UP_SEQ_FEATURE & Domain: BTB & 6 & I.80E-02 & $4.90 \mathrm{E}-0 \mathrm{I}$ \\
\hline INTERPRO & BTB/POZ-like & 6 & 5. $10 \mathrm{E}-02$ & $8.80 \mathrm{E}-0 \mathrm{I}$ \\
\hline INTERPRO & BTB/POZ fold & 6 & $5.30 \mathrm{E}-02$ & $8.40 \mathrm{E}-0 \mathrm{I}$ \\
\hline SMART & Btb & 6 & $8.40 \mathrm{E}-02$ & 8. IOE-0I \\
\hline Annotation cluster 13 & Enrichment score: I.3I & & & \\
\hline INTERPRO & Peptidyl-prolyl cis-trans isomerase, FKBP-type & 3 & $2.10 \mathrm{E}-02$ & $7.60 \mathrm{E}-0 \mathrm{I}$ \\
\hline SP_PIR_KEYWORDS & Rotamase & 3 & $5.90 \mathrm{E}-02$ & 5.00E-0I \\
\hline GOTERM_MF_FAT & Peptidyl-prolyl cis-trans isomerase activity & 3 & $6.50 \mathrm{E}-02$ & $9.50 \mathrm{E}-0 \mathrm{I}$ \\
\hline GOTERM_MF_FAT & cis-trans Isomerase activity & 3 & 7. $10 \mathrm{E}-02$ & $9.30 \mathrm{E}-0 \mathrm{I}$ \\
\hline Annotation cluster I4 & Enrichment score: 1.27 & & & \\
\hline GOTERM_BP_FAT & Regulation of apoptosis & 16 & $5.10 \mathrm{E}-02$ & 5. IOE-0I \\
\hline GOTERM_BP_FAT & Regulation of programmed cell death & 16 & $5.50 \mathrm{E}-02$ & $5.20 \mathrm{E}-0 \mathrm{I}$ \\
\hline GOTERM_BP_FAT & Regulation of cell death & 16 & $5.60 \mathrm{E}-02$ & $5.20 \mathrm{E}-0 \mathrm{I}$ \\
\hline Annotation cluster I5 & Enrichment score: 1.26 & & & \\
\hline GOTERM_BP_FAT & Positive regulation of protein kinase activity & 7 & $4.80 \mathrm{E}-02$ & $5.00 \mathrm{E}-0 \mathrm{I}$ \\
\hline GOTERM_BP_FAT & Positive regulation of kinase activity & 7 & $5.50 \mathrm{E}-02$ & 5.20E-0I \\
\hline GOTERM_BP_FAT & Positive regulation of transferase activity & 7 & $6.40 \mathrm{E}-02$ & 5.40E-0I \\
\hline Annotation cluster 16 & Enrichment score: 1.09 & & & \\
\hline SP_PIR_KEYWORDS & RNA-mediated gene silencing & 3 & $3.30 \mathrm{E}-02$ & 4. IOE-0I \\
\hline GOTERM_BP_FAT & Gene silencing by RNA & 3 & $5.70 \mathrm{E}-02$ & 5.20E-0I \\
\hline GOTERM_BP_FAT & Gene silencing & 3 & I.40E-0I & $6.90 \mathrm{E}-0 \mathrm{I}$ \\
\hline SP_PIR_KEYWORDS & Translation regulation & 3 & I.60E-0I & 7.60E-0I \\
\hline
\end{tabular}

Abbreviations: DAVID, Database for Annotation, Visualization and Integrated Discovery; FDR, false discovery rate.

and lung and respiratory tube development, positive regulation of protein polymerization, positive regulation of protein complex assembly, positive regulation of protein polymerization, and positive regulation of protein complex assembly.

Furthermore, our DAVID analysis revealed that there were nine KEGG pathways significantly enriched in the target list of hsa-miR-181a-5p, based on TarBase (Table 7). These pathways included pathways in cancer pathways (Figure 1), the Wnt signaling pathway (Figure 2), prostate cancer, melanogenesis, cell cycle (Figure 3), hedgehog signaling pathway, p53 signaling pathway (Figure 4), small cell lung cancer, and thyroid cancer.

\section{Validated targets of hsa-miR- I8la-5p based on miRTarBase}

Based on miRTarBase, 241 targets of hsa-miR-181a-5p have been validated with experimental evidence (Table 8). These included ACOT12, AFTPH, AKAP12, AMMECR1, ANKRD1, ANKRD13C, AP1M1, ARF6, ARHGAP12, ARL6IP6, ATF7IP2, ATG10, ATM, ATP6V0E1, ATP8A1, $B A G 2, B C L 2$, BCL2L11, BDNF, BPGM, BRCA1, BRMS1L, BTBD3, C1orf109, C1QTNF9, C8A, CCDC6, CCNG1, $C D 46$, and $C D K N 1 B$. Among these validated targets, only 18 are cancer genes (7.47\%), including ATM, BCL2, CCDC6, CDX2, FBXO11, H3F3B, HOOK3, HOXA11, HRAS, KRAS,
MAP2K1, NOTCH1, NOTCH2, PLAG1, PTPN11, STAG2, $T A F 15$, and TSHR (Table 8). Only half of these cancer genes have been included in TarBase.

As shown in Table 9, our DAVID analysis showed that there were 20 functional clusters that were identified to be enriched with an enrichment score $>1.0$ in the target list of hsa-miR-181a-5p, based on miRTarBase. The functions of these clusters involved negative regulation of transcription, negative regulation of gene expression, negative regulation of nucleobase, nucleoside, nucleotide, and nucleic acid metabolic processes, negative regulation of nitrogen compound metabolic process, negative regulation of macromolecule biosynthetic process, negative regulation

Table 7 The KEGG pathways by DAVID, for the target list of hsa-miR-I8Ia-5p based on TarBase 6.0

\begin{tabular}{lllll}
\hline Signaling pathway & Gene count & $\%$ & P-value & FDR \\
\hline Pathways in cancer & II & 0.5 & $4.50 \mathrm{E}-03$ & $\mathrm{I} .30 \mathrm{E}-0 \mathrm{I}$ \\
Wnt signaling pathway & 8 & 0.4 & $\mathrm{I} .80 \mathrm{E}-03$ & $\mathrm{I} .50 \mathrm{E}-0 \mathrm{I}$ \\
Prostate cancer & 6 & 0.3 & $3.80 \mathrm{E}-03$ & $\mathrm{I} .60 \mathrm{E}-0 \mathrm{I}$ \\
Melanogenesis & 5 & 0.2 & $2.90 \mathrm{E}-02$ & $4.10 \mathrm{E}-0 \mathrm{I}$ \\
Cell cycle & 5 & 0.2 & $5.90 \mathrm{E}-02$ & $5.00 \mathrm{E}-0 \mathrm{I}$ \\
Hedgehog signaling pathway & 4 & 0.2 & $2.80 \mathrm{E}-02$ & $4.70 \mathrm{E}-0 \mathrm{I}$ \\
P53 signaling pathway & 4 & 0.2 & $4.60 \mathrm{E}-02$ & $4.50 \mathrm{E}-0 \mathrm{I}$ \\
Small cell lung cancer & 4 & 0.2 & $7.60 \mathrm{E}-02$ & $5.50 \mathrm{E}-0$ I \\
Thyroid cancer & 3 & 0.1 & $4.50 \mathrm{E}-02$ & $5.00 \mathrm{E}-0 \mathrm{I}$ \\
\hline
\end{tabular}

Abbreviations: DAVID, Database for Annotation, Visualization and Integrated Discovery; FDR, false discovery rate; KEGG, Kyoto Encyclopedia of Genes and Genomes. 


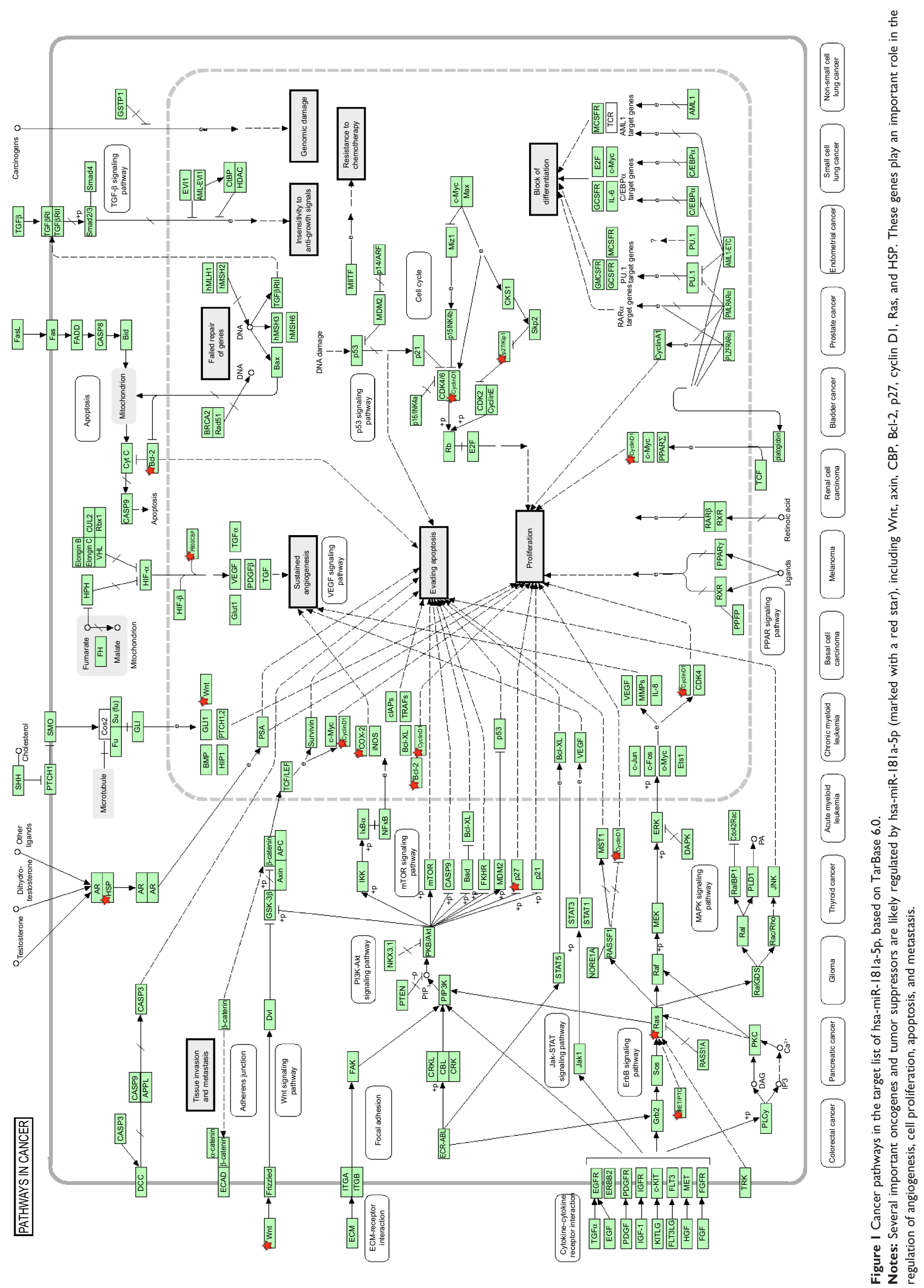




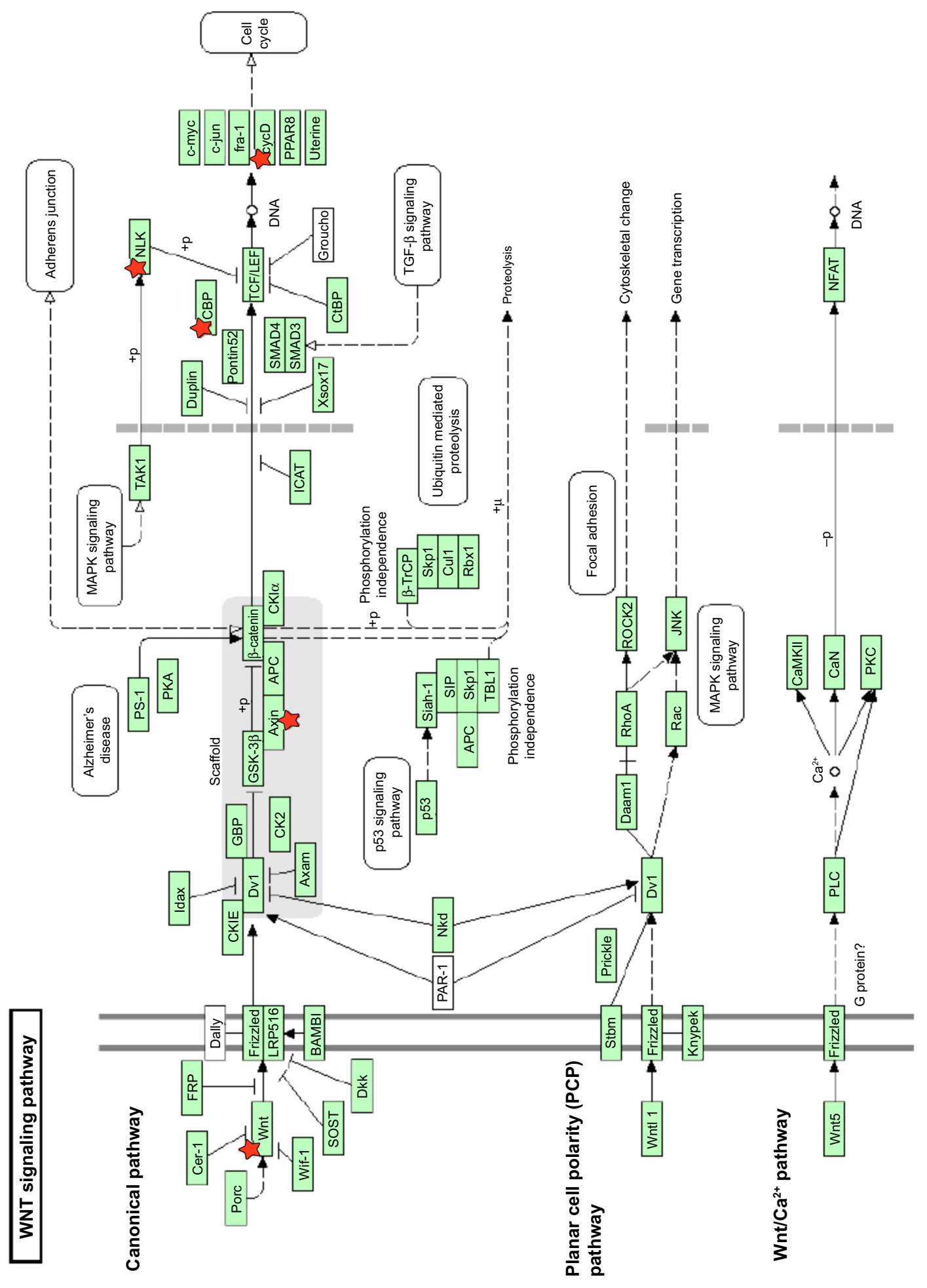

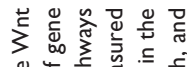

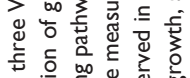

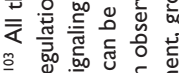

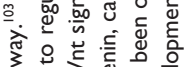

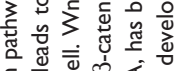

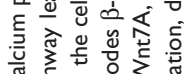

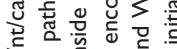

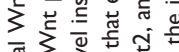

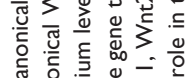

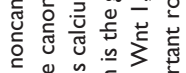

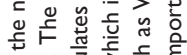

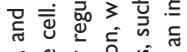

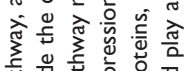

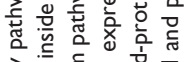

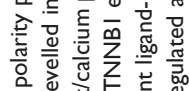

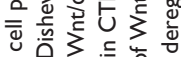

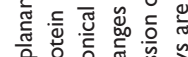

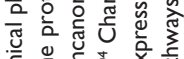

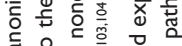

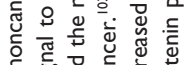

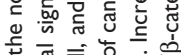

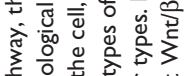

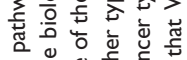

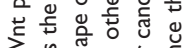

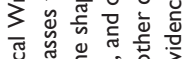

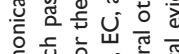

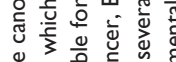

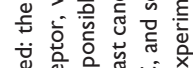

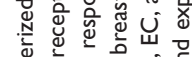

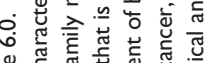

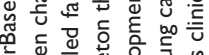

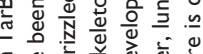

ᄃ。

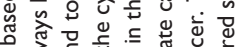

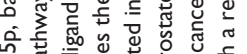

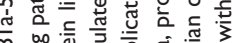

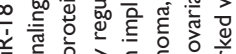

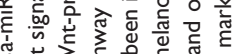

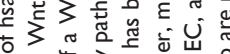

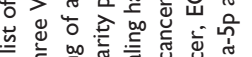

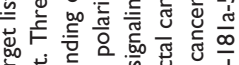

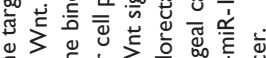

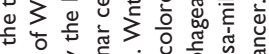

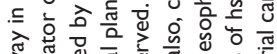

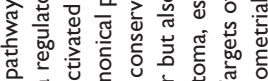

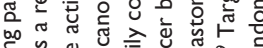

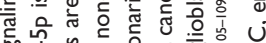

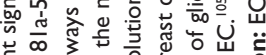

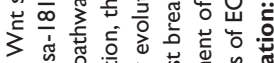

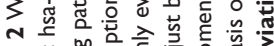

Minn 


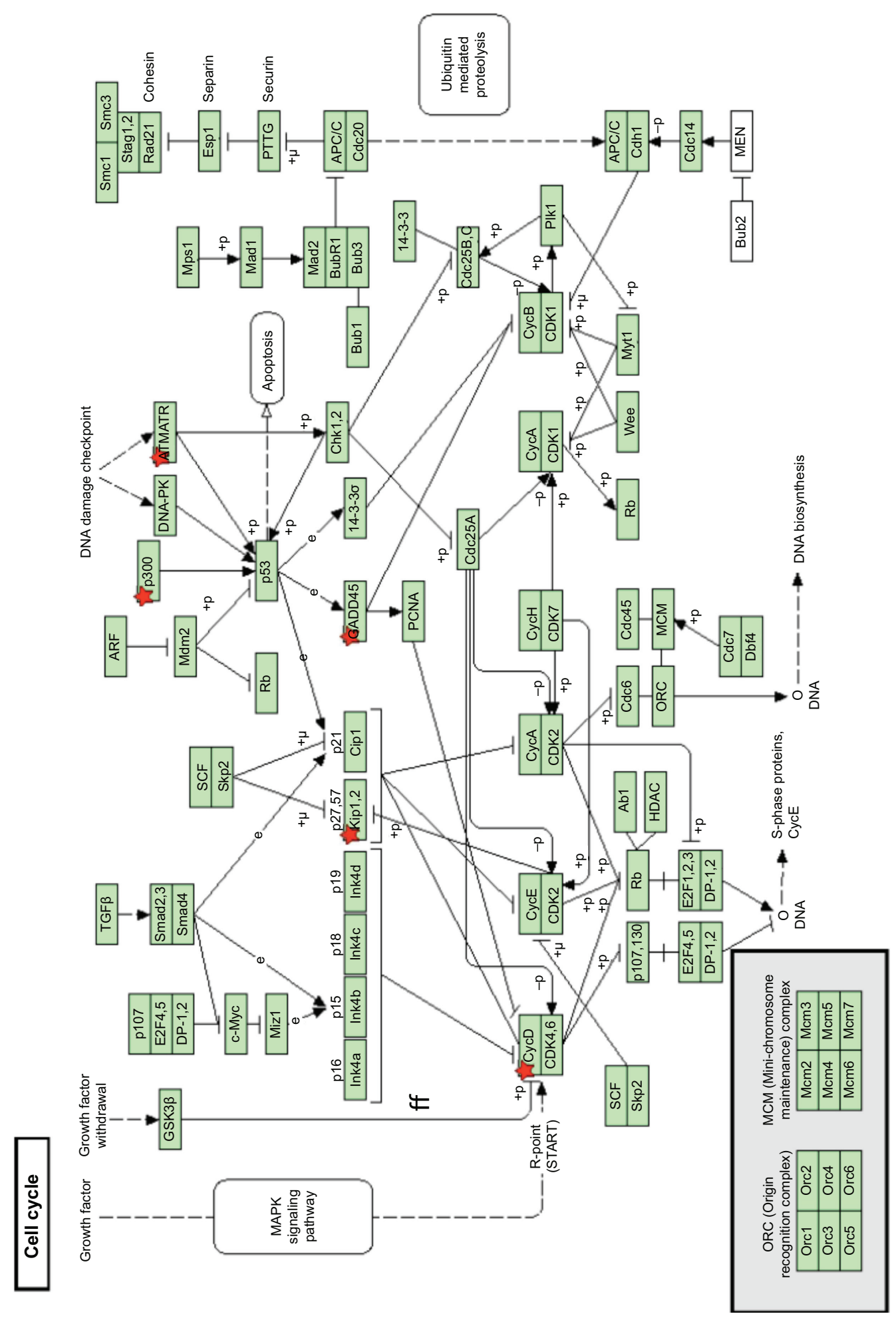

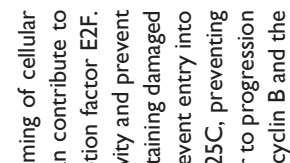

告记

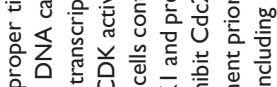

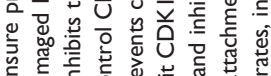

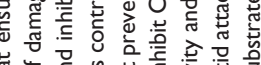

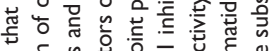

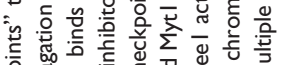

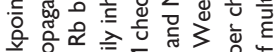

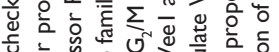

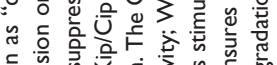

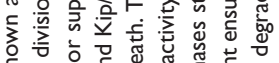

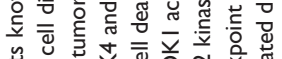

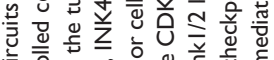

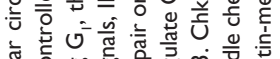

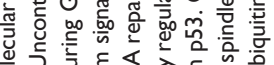

이에

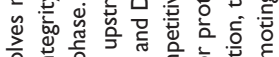

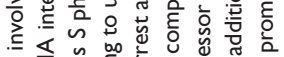

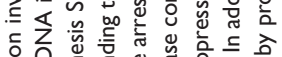

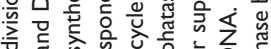

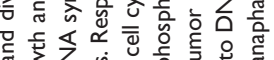

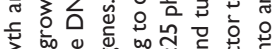

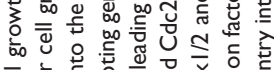

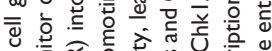

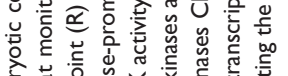

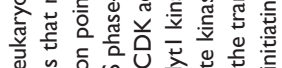

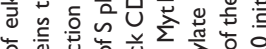

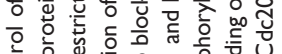

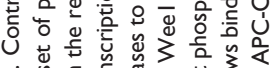

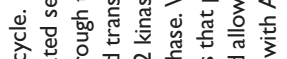

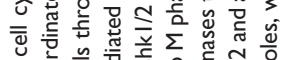

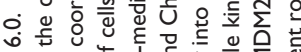

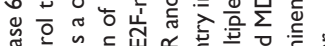

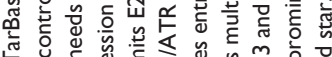

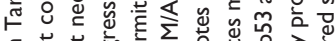

ᄃ

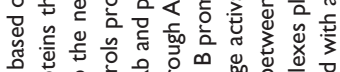

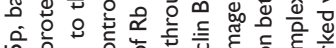

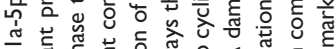

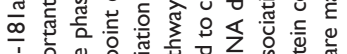

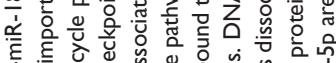

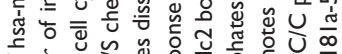

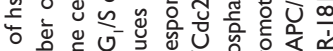

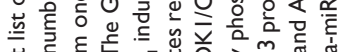

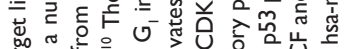

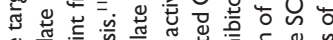

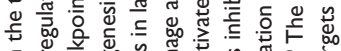
든

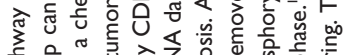

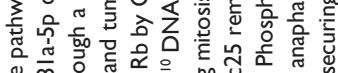
는 仓े

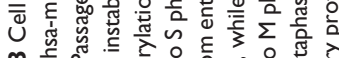
InIm MWM 


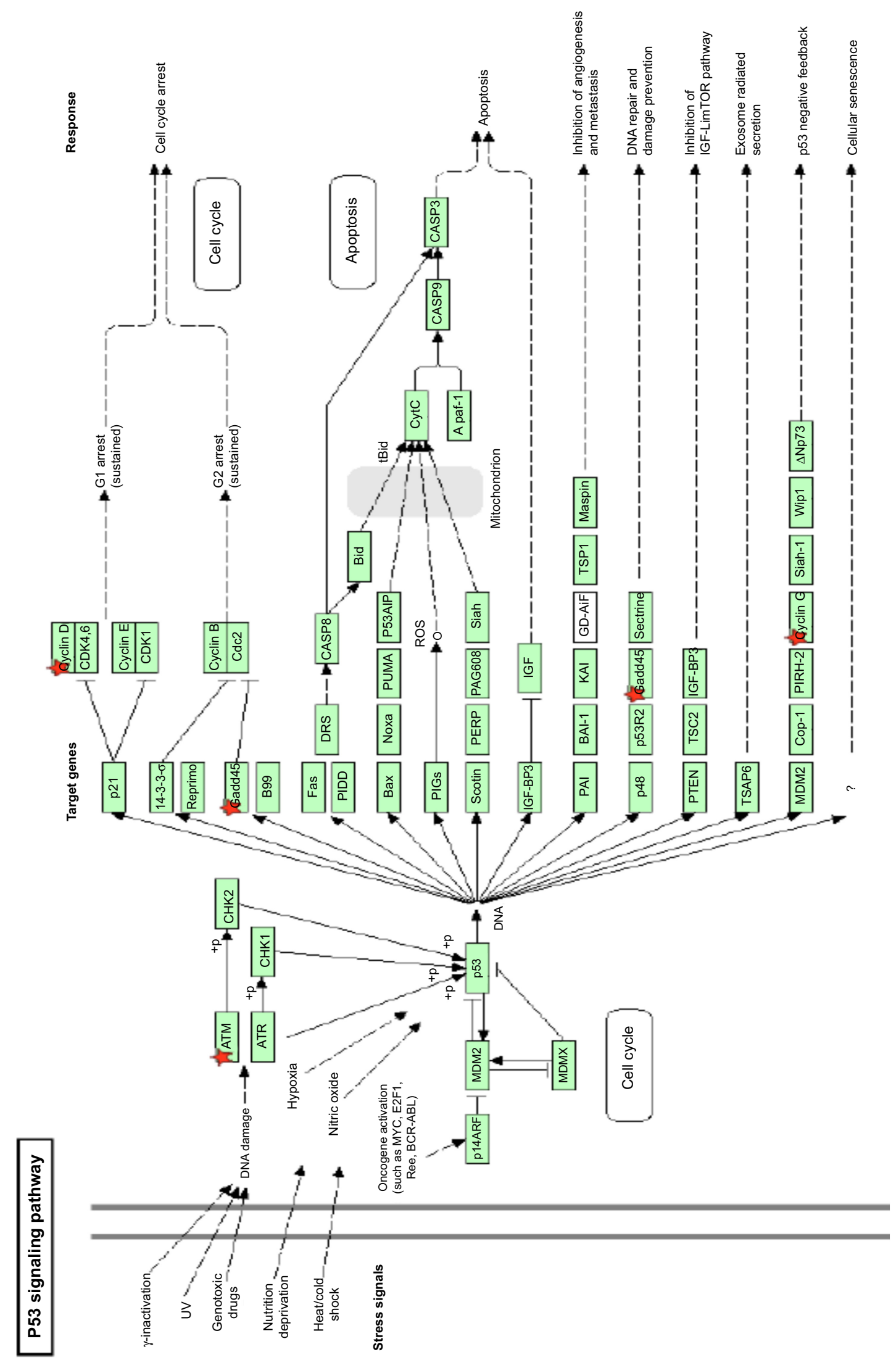

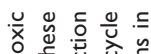

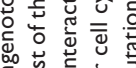

o.

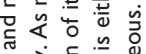

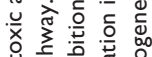

范芷总

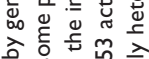

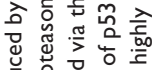

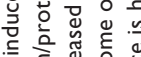

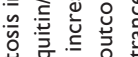

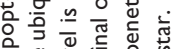

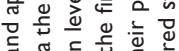

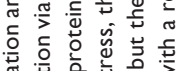

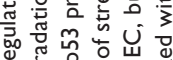

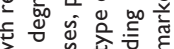

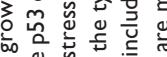

ब于

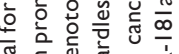

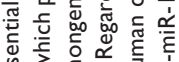

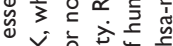

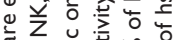

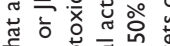

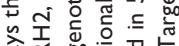

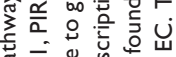

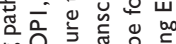

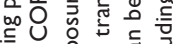

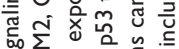

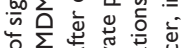

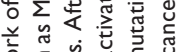

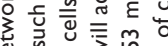

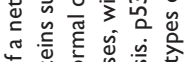

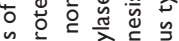

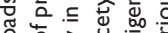

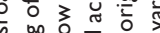

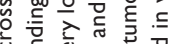

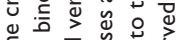

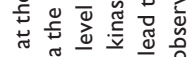

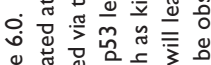

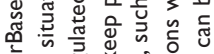

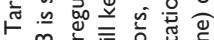

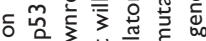

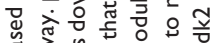

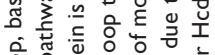

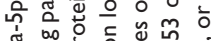

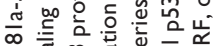

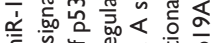

离㘦

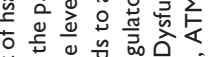

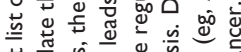

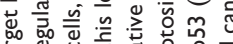

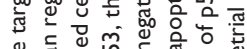

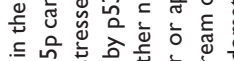

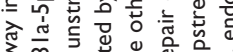

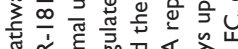

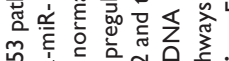

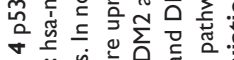

Uin 


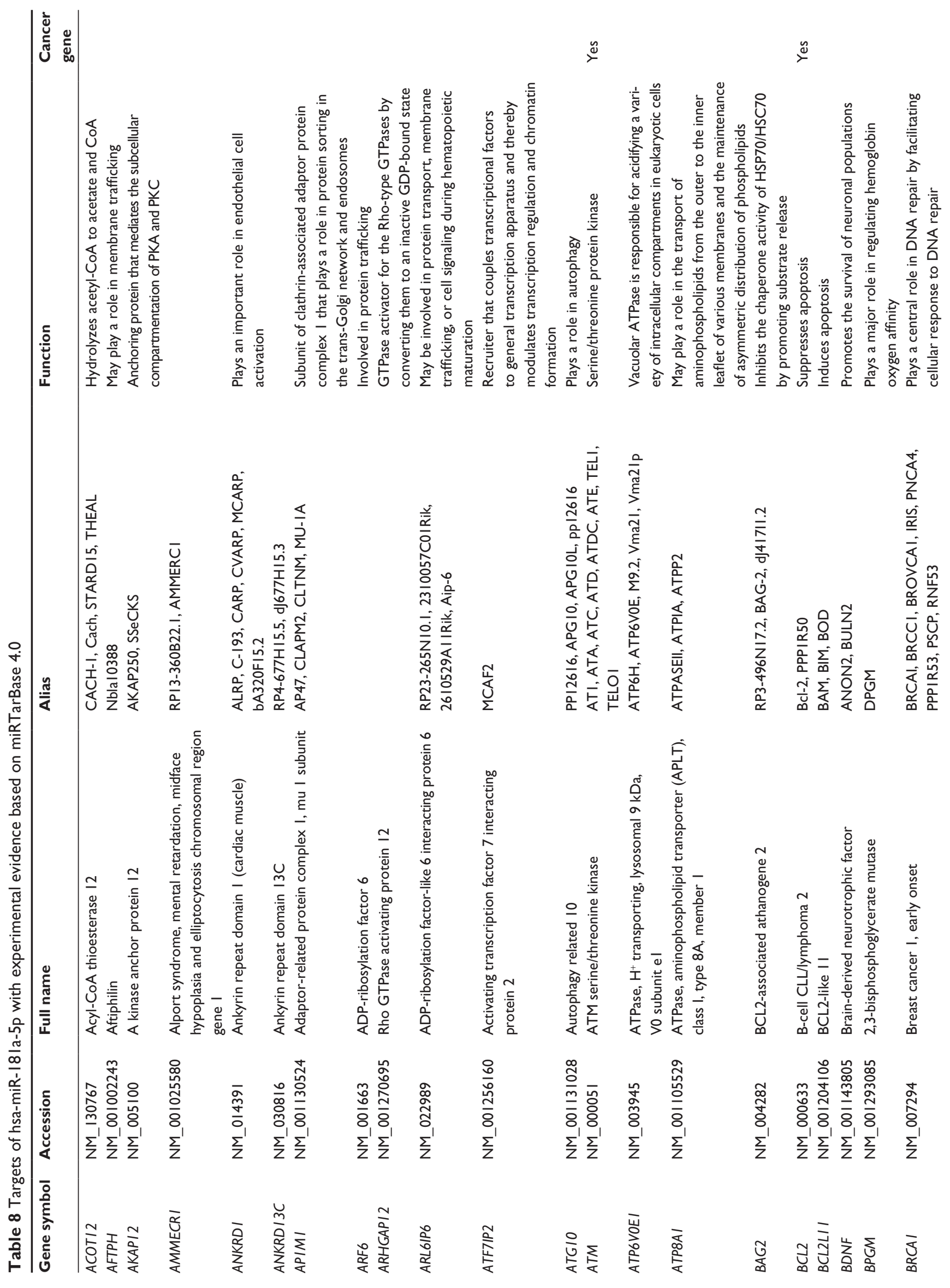



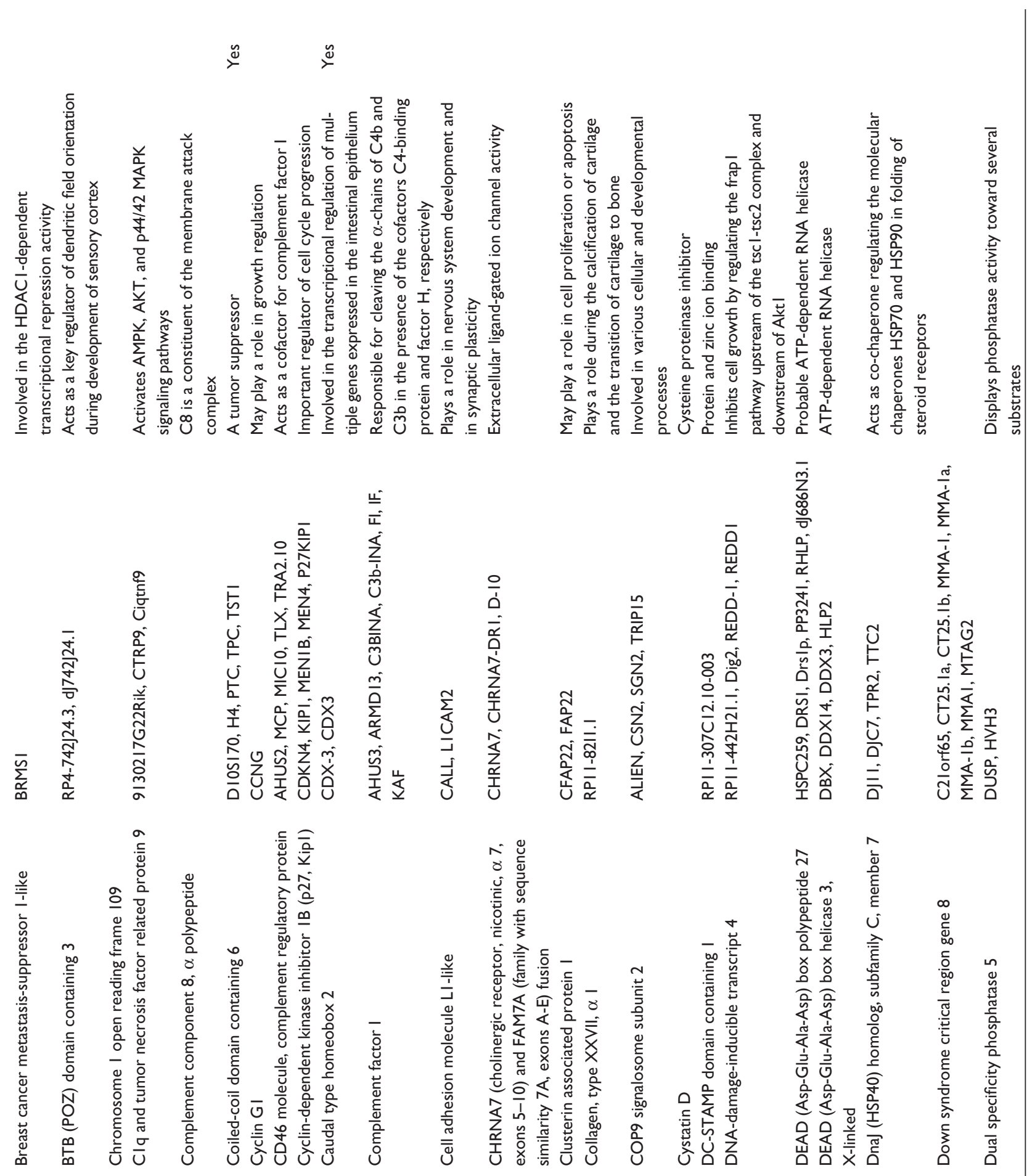

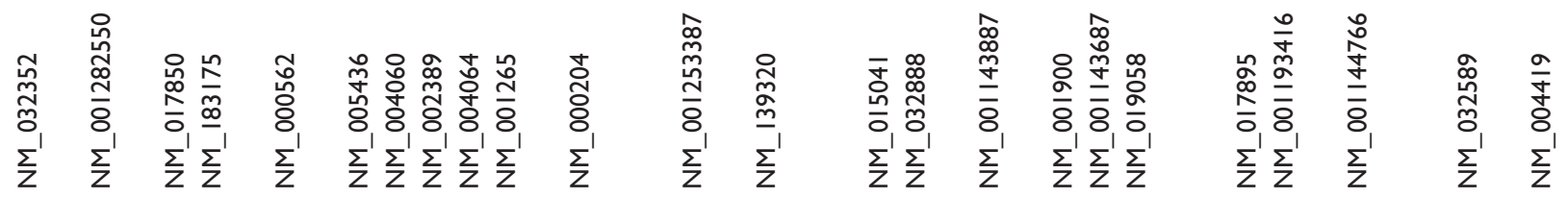

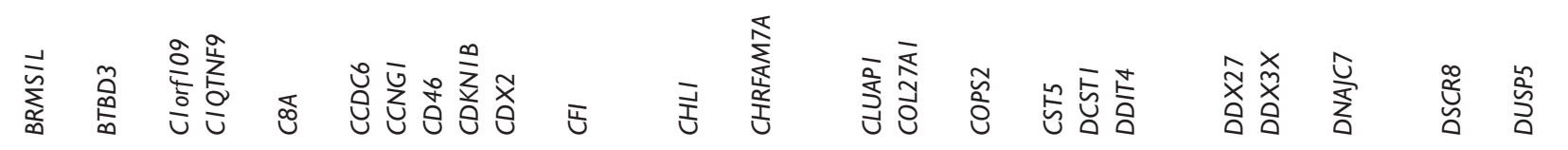




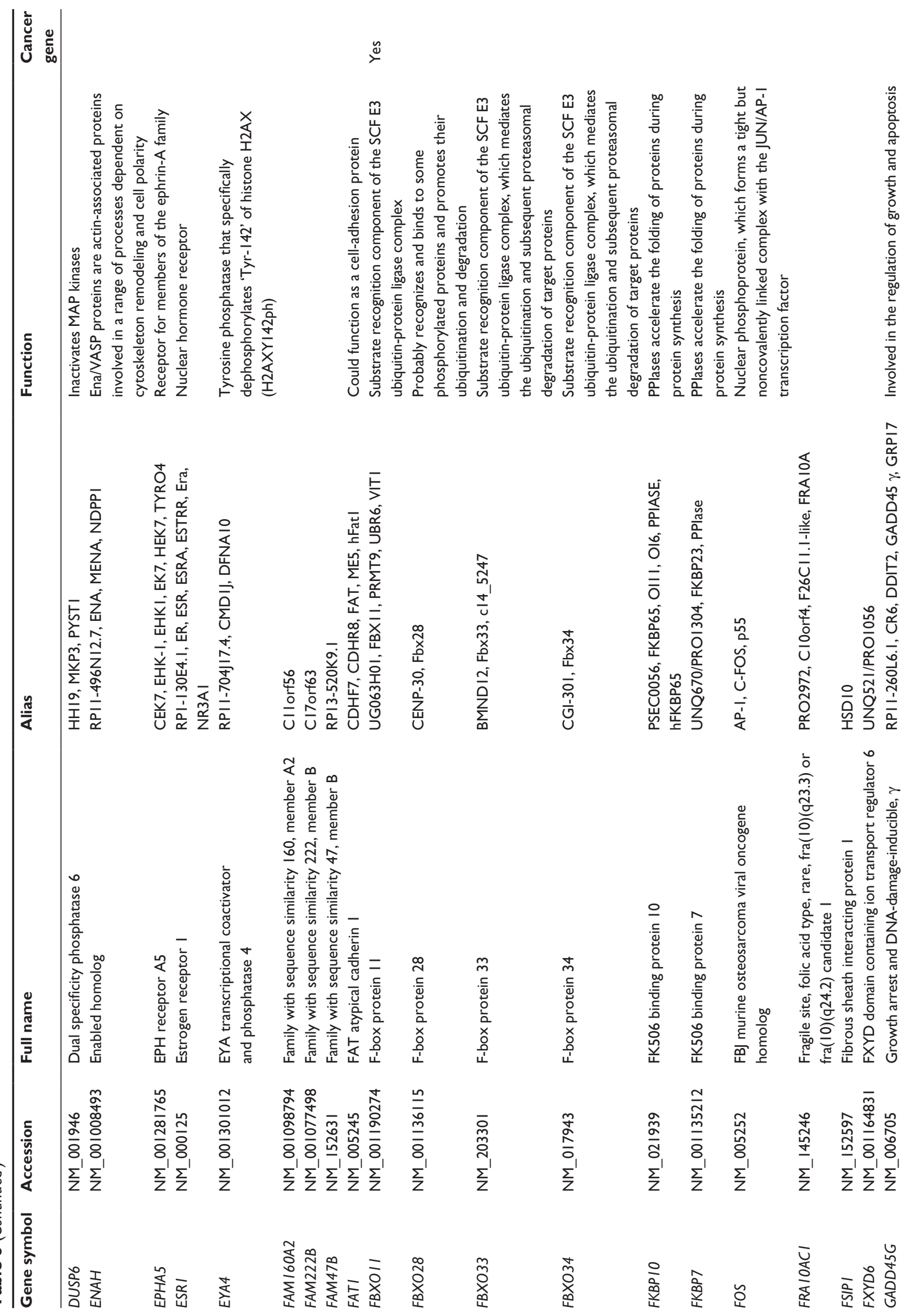



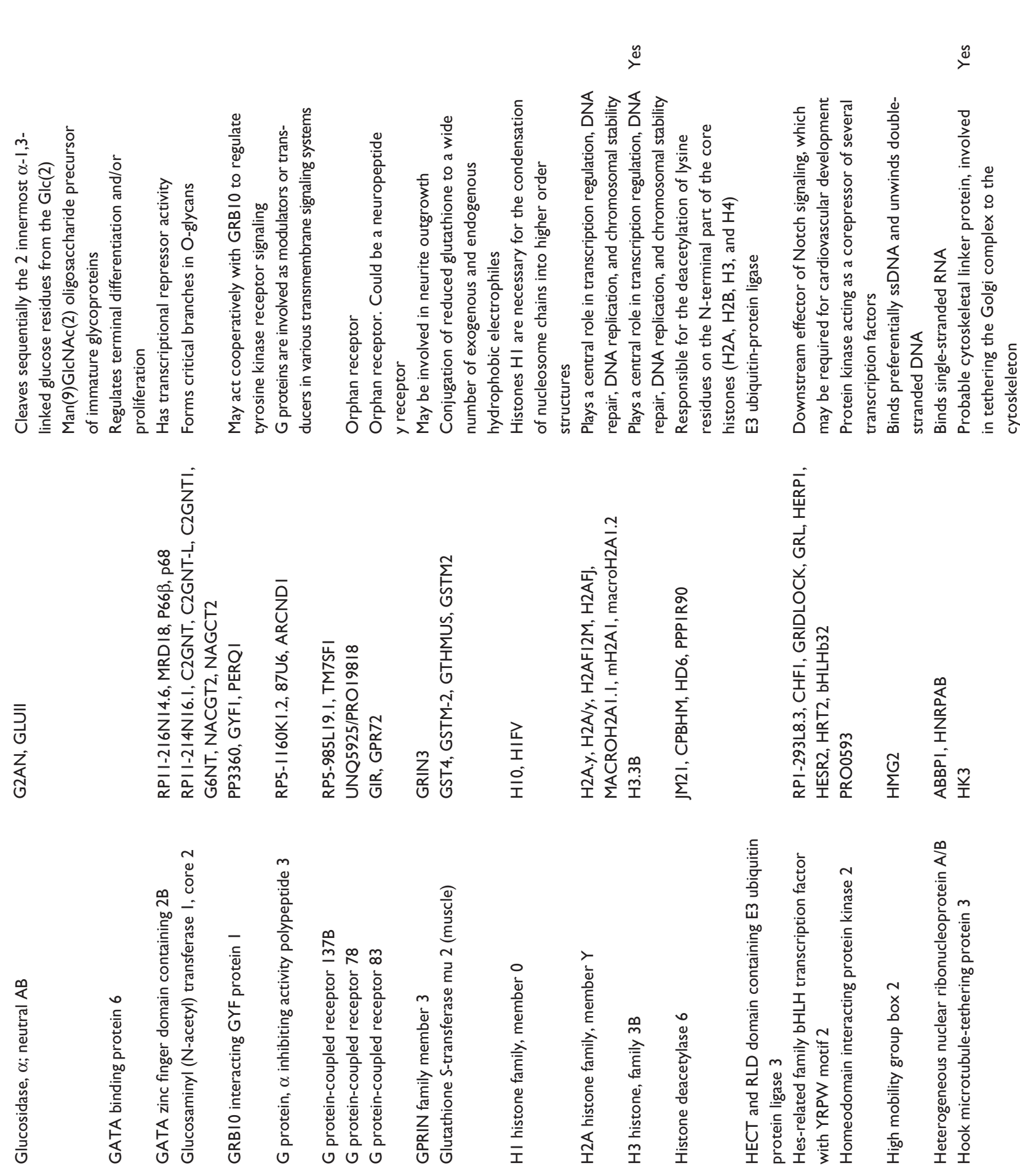

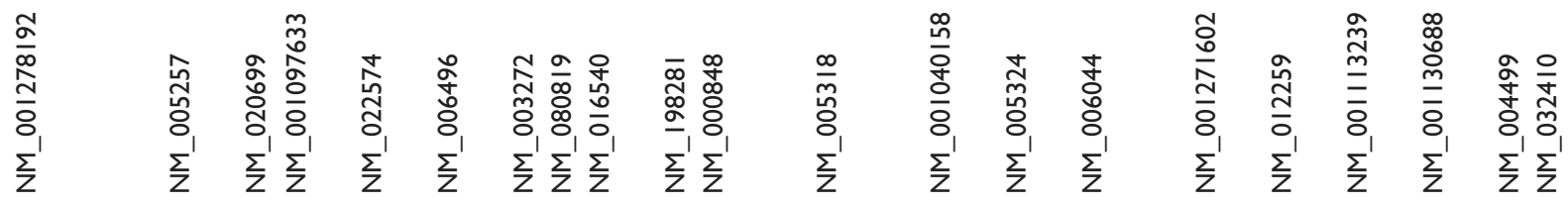

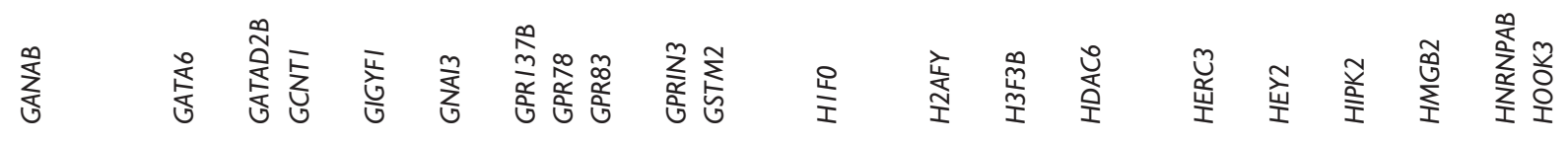




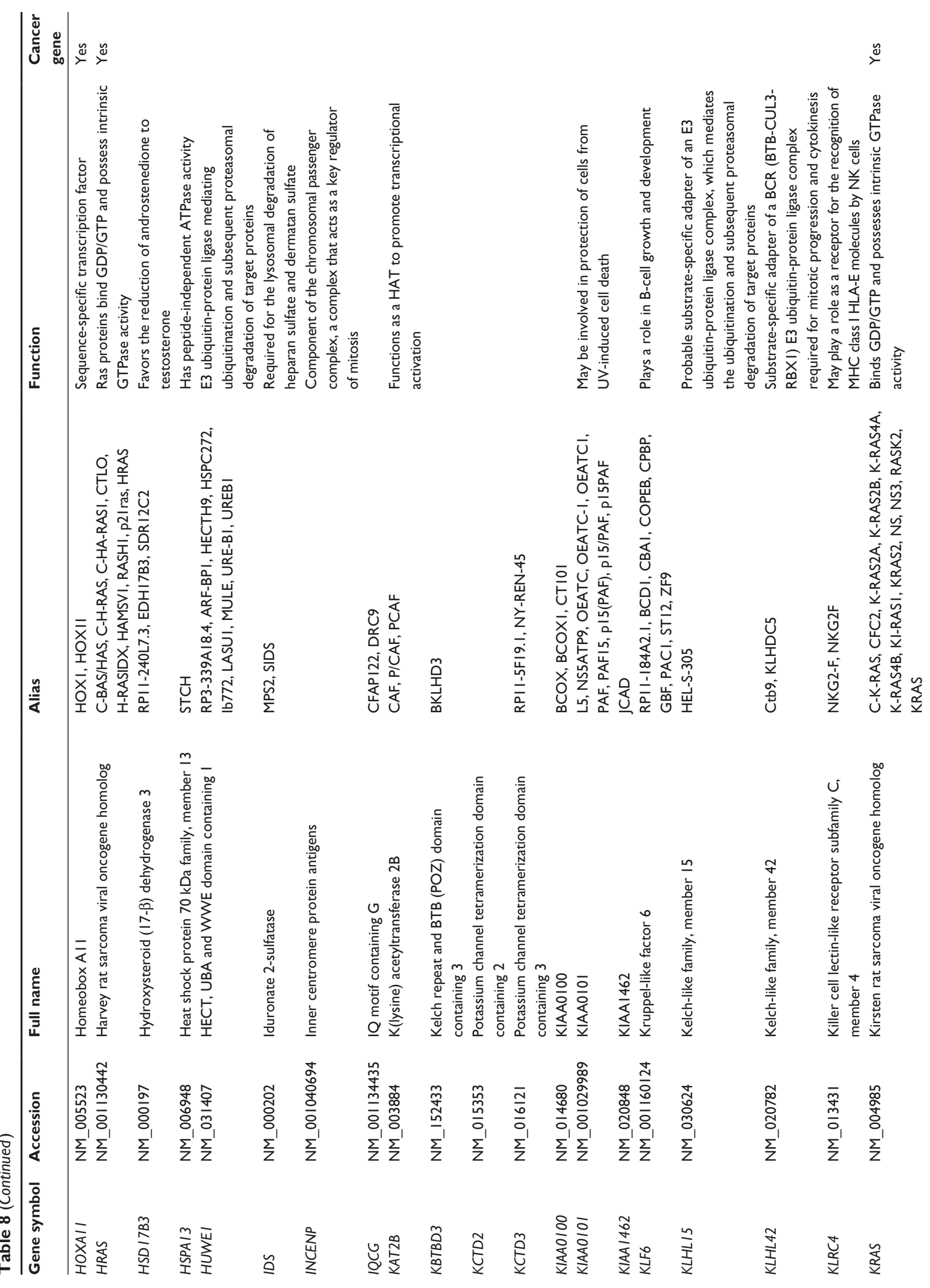




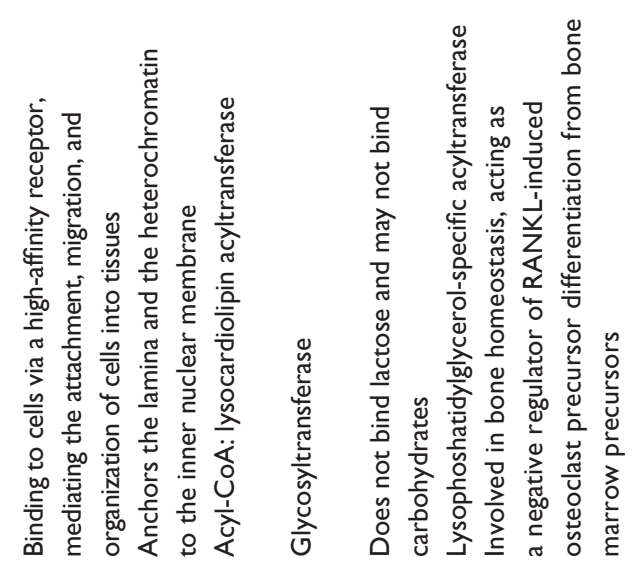

$\overbrace{}^{\stackrel{y}{x}}$

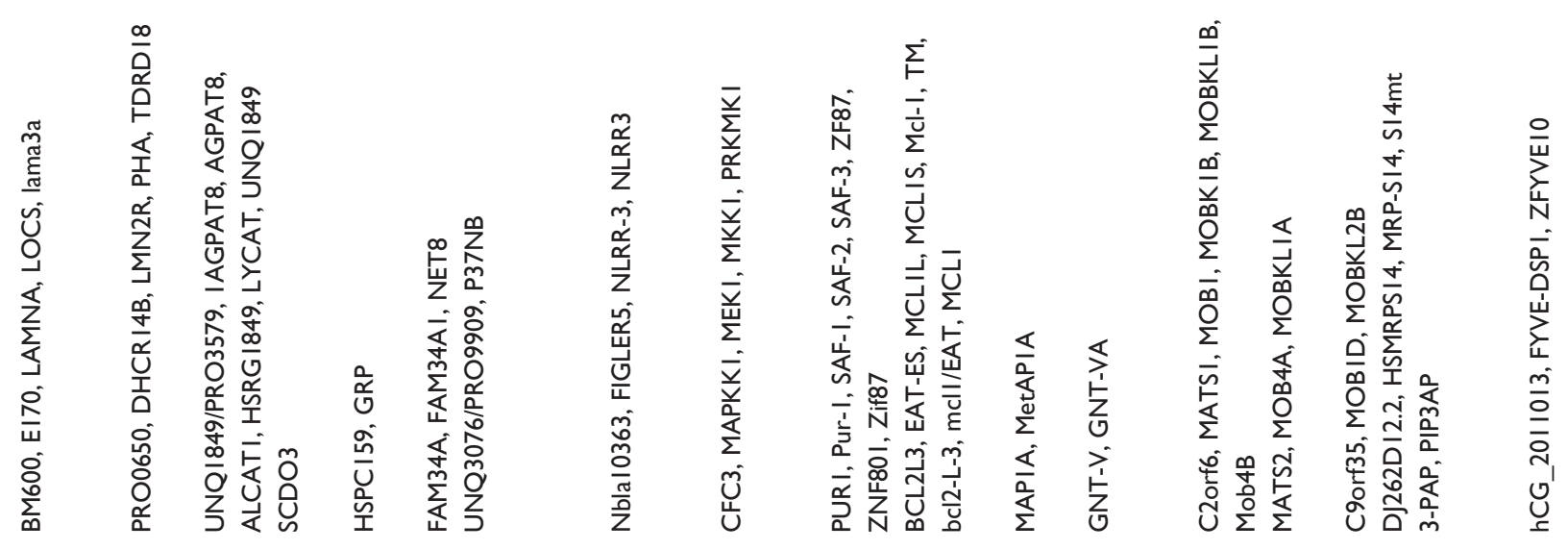

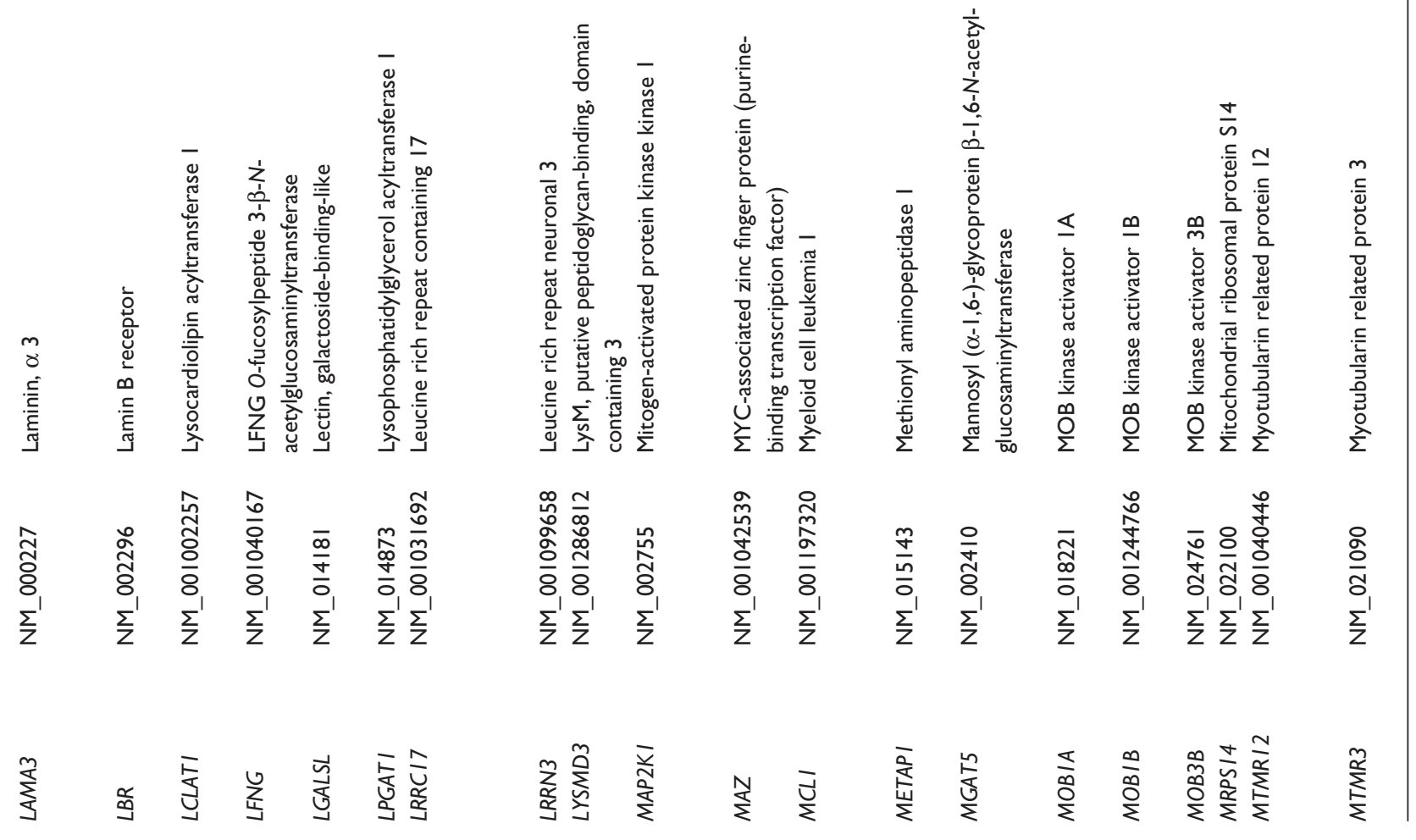




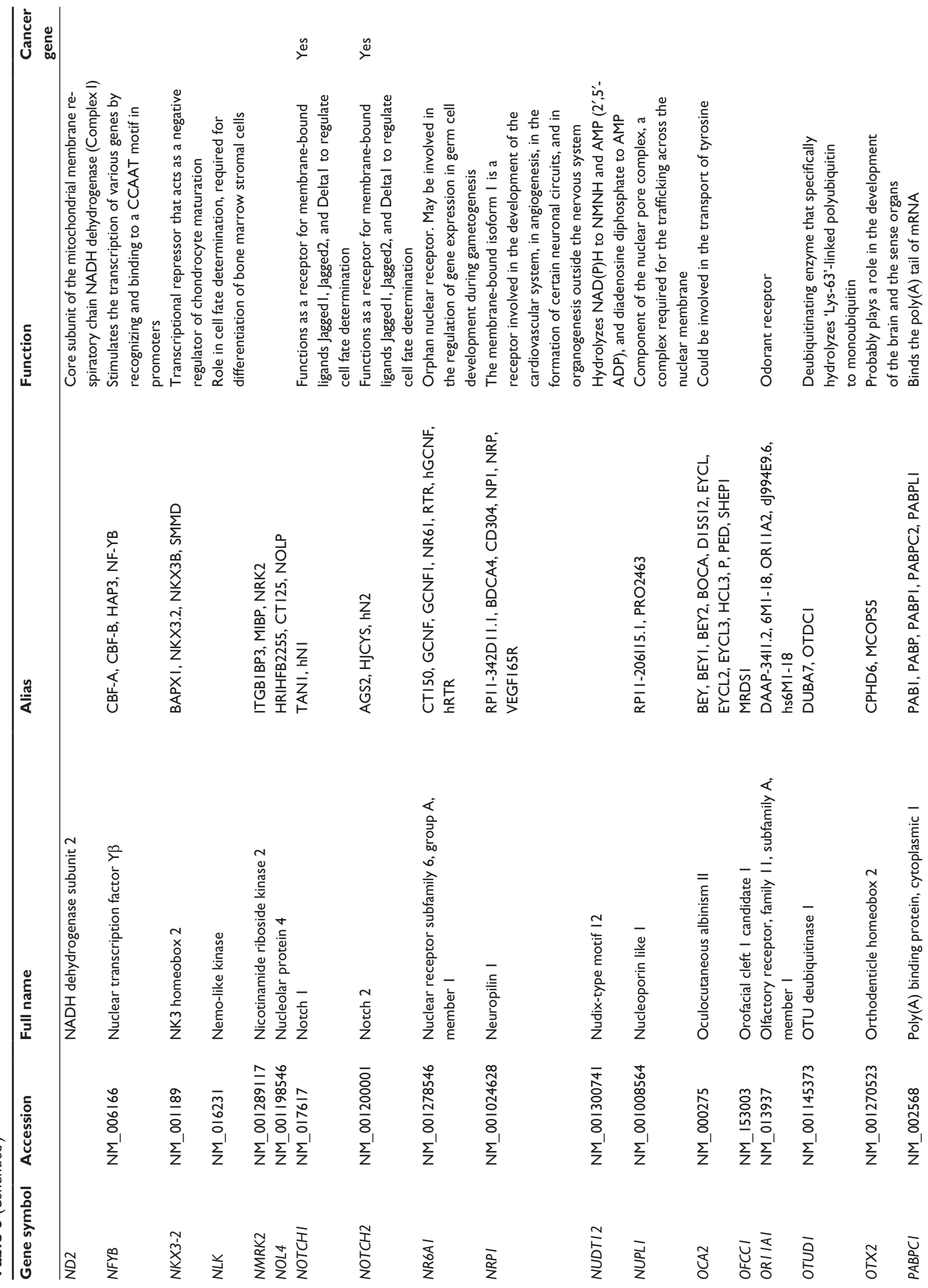



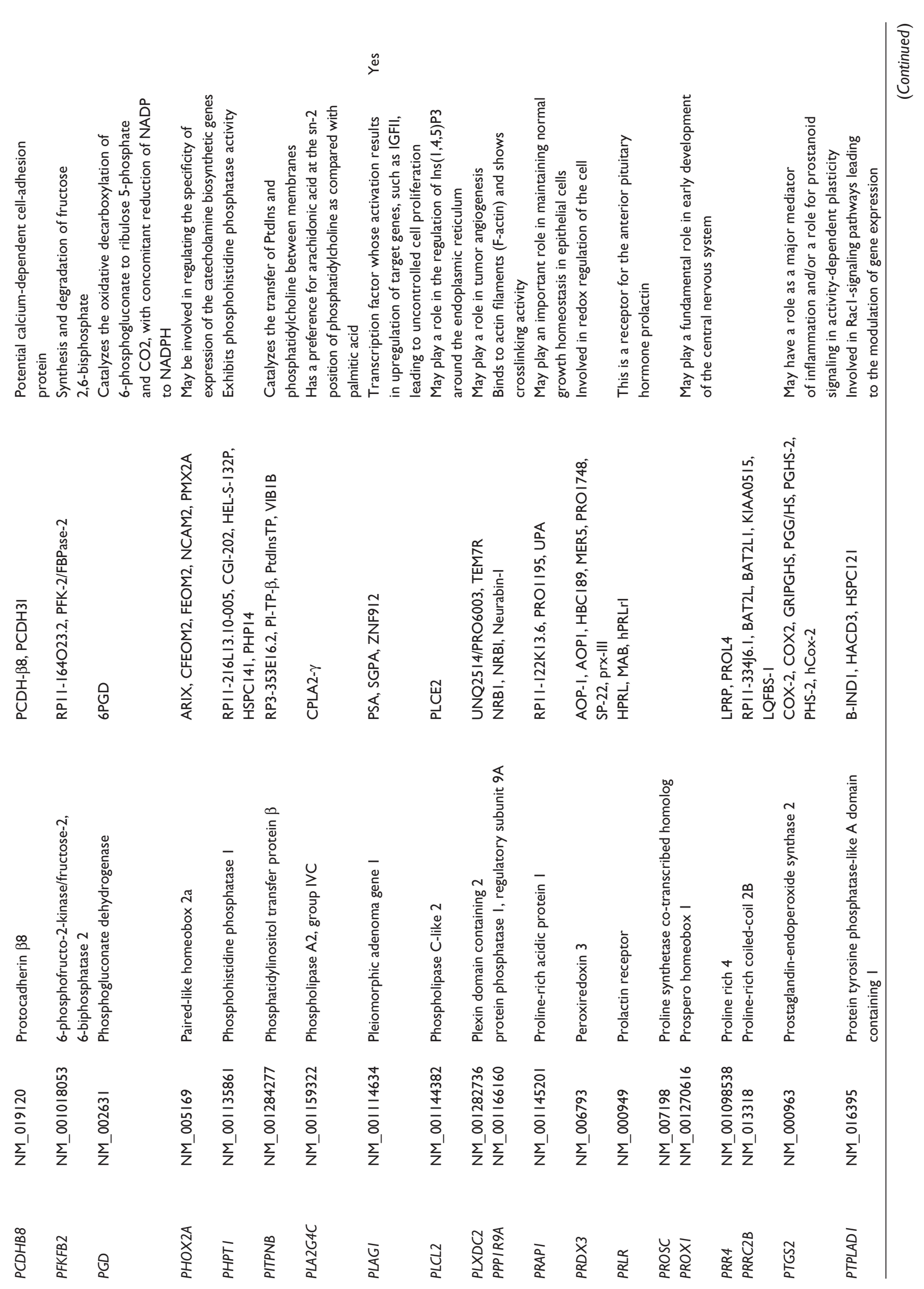


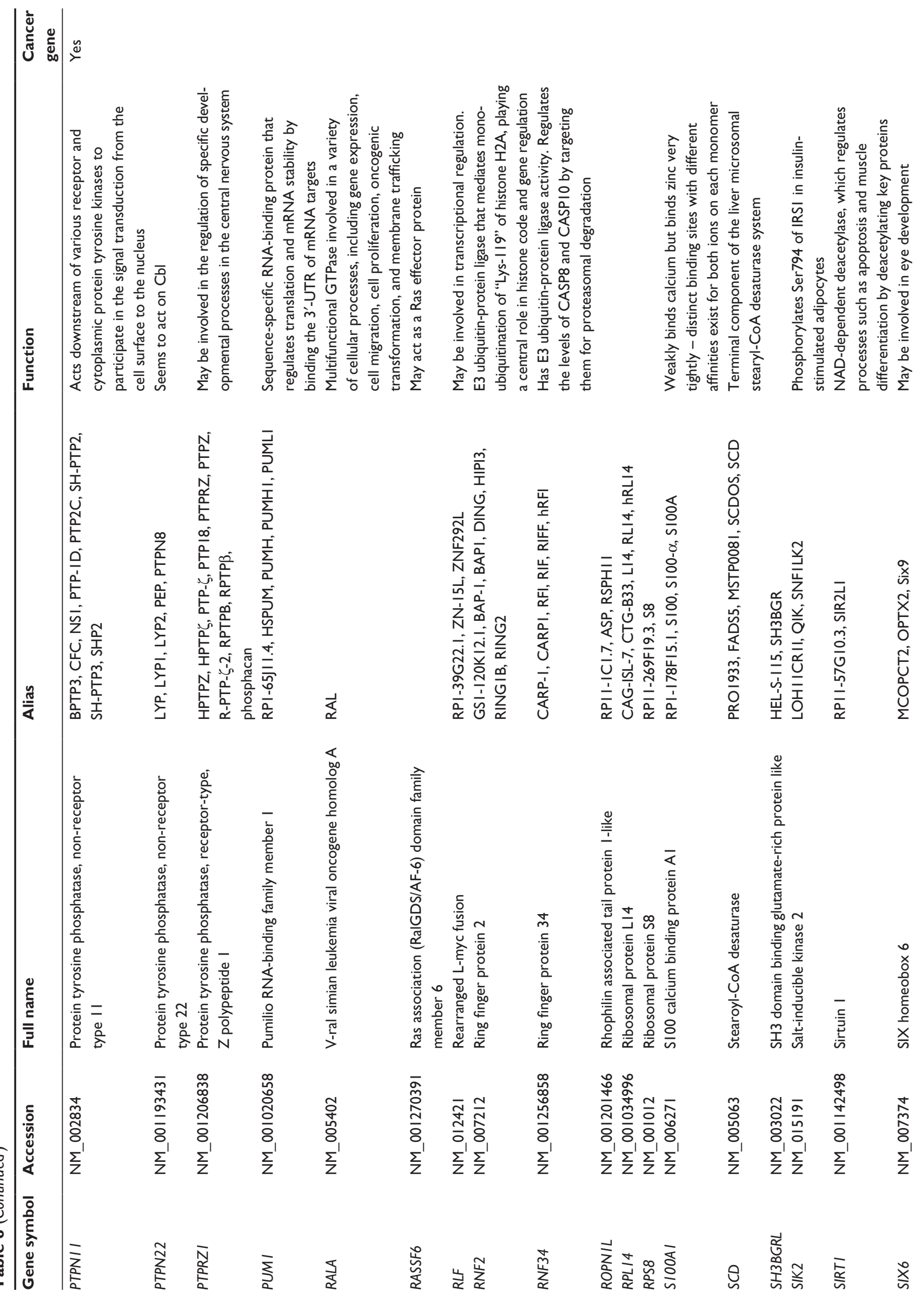



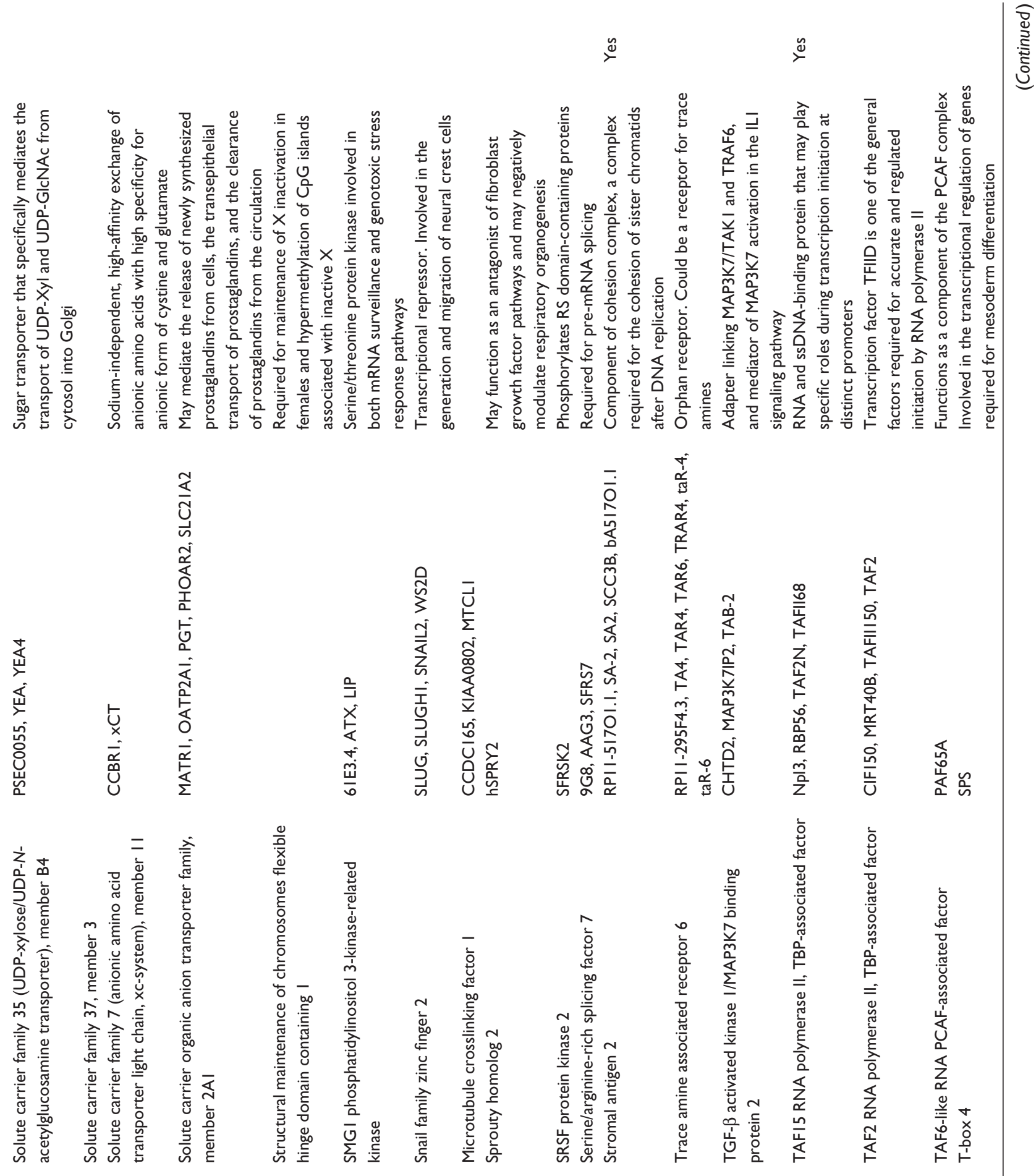

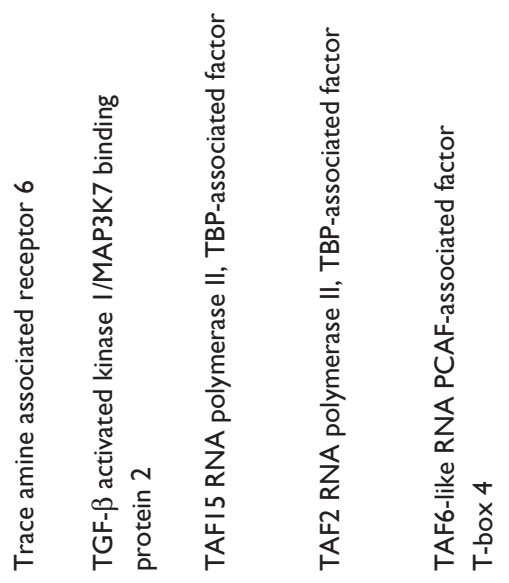

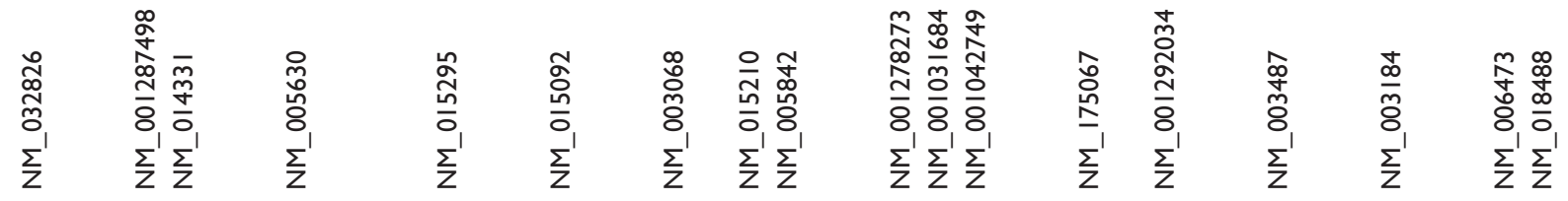

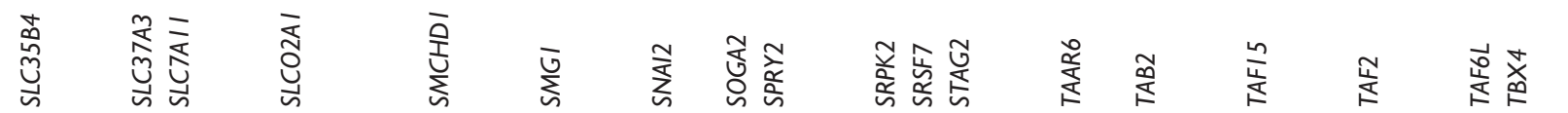




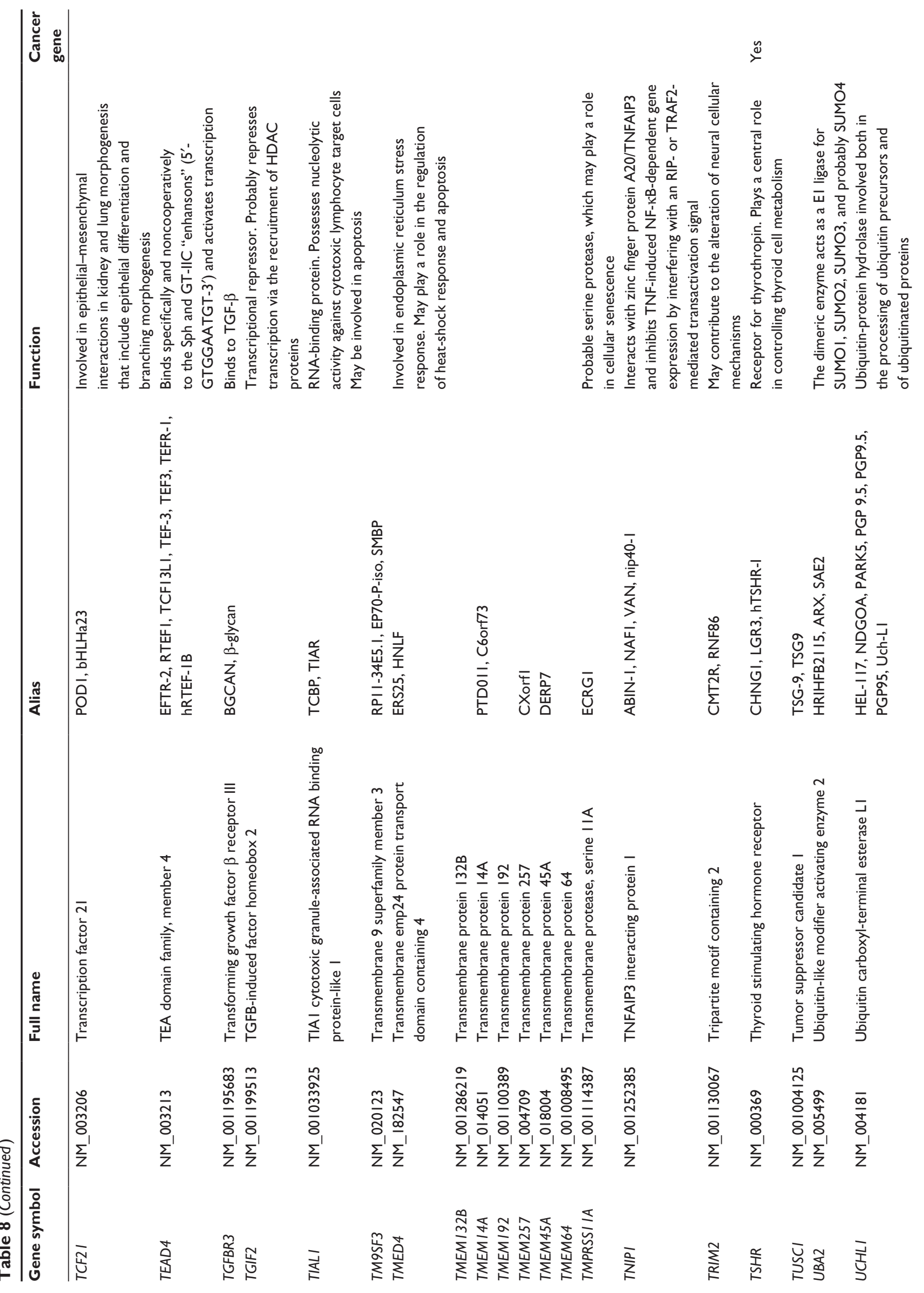



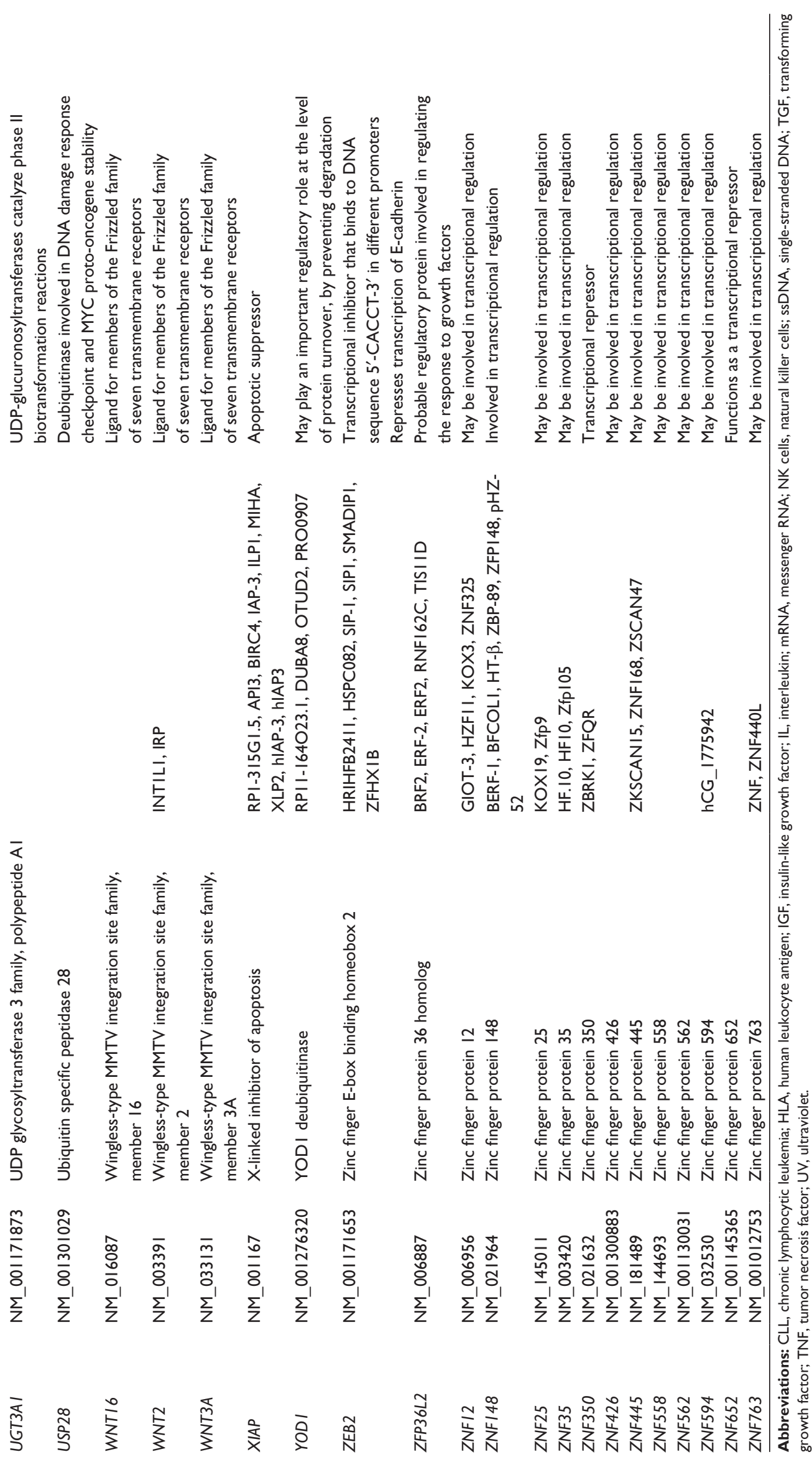
Table 9 The top enriched clusters (enrich score $>I$ ) by DAVID for the targets of hsa-miR-I8Ia-5p from miRTarBase 4.0

\begin{tabular}{|c|c|c|c|c|}
\hline Category & Term & Gene count & $P$-value & FDR \\
\hline Annotation cluster I & Enrichment score: 2.9 & & & \\
\hline GOTERM_BP_FAT & Negative regulation of transcription & 17 & 3.10E-04 & $7.80 \mathrm{E}-02$ \\
\hline GOTERM_BP_FAT & Negative regulation of gene expression & 17 & $8.50 \mathrm{E}-04$ & $9.90 \mathrm{E}-02$ \\
\hline GOTERM_BP_FAT & $\begin{array}{l}\text { Negative regulation of nucleobase, nucleoside, nucleotide and } \\
\text { nucleic acid metabolic process }\end{array}$ & 17 & I.00E-03 & I.00E-0I \\
\hline GOTERM_BP_FAT & Negative regulation of nitrogen compound metabolic process & 17 & I.20E-03 & I.00E-0I \\
\hline GOTERM_BP_FAT & Negative regulation of macromolecule biosynthetic process & 17 & $2.00 \mathrm{E}-03$ & I.20E-0I \\
\hline GOTERM_BP_FAT & Negative regulation of cellular biosynthetic process & 17 & $2.50 \mathrm{E}-03$ & I.20E-0। \\
\hline GOTERM_BP_FAT & Negative regulation of biosynthetic process & 17 & $3.10 \mathrm{E}-03$ & I.20E-0 I \\
\hline Annotation cluster 2 & Enrichment score: $\mathbf{2 . 8 6}$ & & & \\
\hline GOTERM_BP_FAT & Regulation of phosphorylation & 16 & I.10E-03 & I.00E-0I \\
\hline GOTERM_BP_FAT & Regulation of phosphorus metabolic process & 16 & I.60E-03 & I.IOE-0I \\
\hline GOTERM_BP_FAT & Regulation of phosphate metabolic process & 16 & I.60E-03 & I.IOE-0I \\
\hline Annotation cluster 3 & Enrichment score: 2.66 & & & \\
\hline GOTERM_BP_FAT & Lung development & 7 & I.80E-03 & I.IOE-0I \\
\hline GOTERM_BP_FAT & Respiratory tube development & 7 & 2. $10 \mathrm{E}-03$ & I.20E-0I \\
\hline GOTERM_BP_FAT & Respiratory system development & 7 & $2.80 \mathrm{E}-03$ & I.20E-0 I \\
\hline Annotation cluster 4 & Enrichment score: 2.38 & & & \\
\hline GOTERM_BP_FAT & Positive regulation of transcription, DNA-dependent & 16 & I.30E-03 & I.00E-0I \\
\hline GOTERM_BP_FAT & Positive regulation of transcription & 16 & $6.50 \mathrm{E}-03$ & I.70E-0 I \\
\hline GOTERM_BP_FAT & Positive regulation of gene expression & 16 & $8.50 \mathrm{E}-03$ & I.80E-0 I \\
\hline Annotation cluster 5 & Enrichment score: 2.37 & & & \\
\hline GOTERM_BP_FAT & Mesenchymal cell differentiation & 5 & $4.20 \mathrm{E}-03$ & I.50E-0 I \\
\hline GOTERM_BP_FAT & Mesenchymal cell development & 5 & 4.20E-03 & I.50E-0 I \\
\hline GOTERM_BP_FAT & Mesenchyme development & 5 & 4.50E-03 & I.50E-0 I \\
\hline Annotation cluster 6 & Enrichment score: 2.16 & & & \\
\hline GOTERM_BP_FAT & Negative regulation of apoptosis & 12 & $6.40 \mathrm{E}-03$ & I.70E-0। \\
\hline GOTERM_BP_FAT & Negative regulation of programmed cell death & 12 & $7.10 \mathrm{E}-03$ & I.70E-0 I \\
\hline GOTERM_BP_FAT & Negative regulation of cell death & 12 & $7.20 \mathrm{E}-03$ & I.70E-0I \\
\hline Annotation cluster 7 & Enrichment score: 2.12 & & & \\
\hline BIOCARTA & IGF-I signaling pathway & 4 & $7.60 \mathrm{E}-03$ & $3.80 \mathrm{E}-0 \mathrm{I}$ \\
\hline BIOCARTA & IL-6 signaling pathway & 4 & $7.60 \mathrm{E}-03$ & $3.80 \mathrm{E}-0 \mathrm{I}$ \\
\hline BIOCARTA & Insulin signaling pathway & 4 & $7.60 \mathrm{E}-03$ & $3.80 \mathrm{E}-0 \mathrm{I}$ \\
\hline Annotation cluster 8 & Enrichment score: 2.03 & & & \\
\hline SP_PIR_KEYWORDS & Ubl conjugation pathway & 15 & $2.80 \mathrm{E}-03$ & $9.30 \mathrm{E}-02$ \\
\hline GOTERM_BP_FAT & Modification-dependent macromolecule catabolic process & 15 & I.70E-02 & 2.40E-0I \\
\hline GOTERM_BP_FAT & Modification-dependent protein catabolic process & 15 & I.70E-02 & 2.40E-0I \\
\hline Annotation cluster 9 & Enrichment score: 2 & & & \\
\hline GOTERM_CC_FAT & Intracellular organelle lumen & 33 & $7.40 \mathrm{E}-03$ & $3.90 \mathrm{E}-0 \mathrm{I}$ \\
\hline GOTERM_CC_FAT & Organelle lumen & 33 & $1.00 \mathrm{E}-02$ & 4.20E-0I \\
\hline GOTERM_CC_FAT & Membrane-enclosed lumen & 33 & I.30E-02 & $4.50 \mathrm{E}-0 \mathrm{I}$ \\
\hline Annotation cluster 10 & Enrichment score: 1.9 & & & \\
\hline GOTERM_BP_FAT & Proteolysis involved in cellular protein catabolic process & 16 & I.I0E-02 & $2.00 \mathrm{E}-0 \mathrm{I}$ \\
\hline GOTERM_BP_FAT & Cellular protein catabolic process & 16 & I.20E-02 & $2.00 \mathrm{E}-0 \mathrm{I}$ \\
\hline GOTERM_BP_FAT & Protein catabolic process & 16 & I.50E-02 & $2.20 \mathrm{E}-0 \mathrm{I}$ \\
\hline Annotation cluster I I & Enrichment score: 1.86 & & & \\
\hline INTERPRO & Zinc finger, $\mathrm{C} 2 \mathrm{H} 2$-type & 19 & $7.50 \mathrm{E}-03$ & $6.70 \mathrm{E}-0 \mathrm{I}$ \\
\hline INTERPRO & Zinc finger, $\mathrm{C} 2 \mathrm{H} 2$-like & 19 & $8.80 \mathrm{E}-03$ & 6.30E-0I \\
\hline SMART & Zinc finger_C2H2 & 19 & 4.00E-02 & $9.00 \mathrm{E}-0 \mathrm{I}$ \\
\hline Annotation cluster 12 & Enrichment score: 1.76 & & & \\
\hline GOTERM_BP_FAT & Regulation of apoptosis & 19 & I.60E-02 & 2.30E-0I \\
\hline GOTERM_BP_FAT & Regulation of programmed cell death & 19 & I.80E-02 & $2.40 \mathrm{E}-0 \mathrm{I}$ \\
\hline GOTERM_BP_FAT & Regulation of cell death & 19 & I.80E-02 & $2.40 \mathrm{E}-0 \mathrm{I}$ \\
\hline Annotation cluster 13 & Enrichment score: I.75 & & & \\
\hline GOTERM_BP_FAT & Determination of left/right symmetry & 4 & I.70E-02 & 2.40E-0 I \\
\hline GOTERM_BP_FAT & Determination of symmetry & 4 & I.80E-02 & 2.40E-0I \\
\hline GOTERM_BP_FAT & Determination of bilateral symmetry & 4 & $1.80 \mathrm{E}-02$ & $2.40 \mathrm{E}-0 \mathrm{I}$ \\
\hline
\end{tabular}


Table 9 (Continued)

\begin{tabular}{|c|c|c|c|c|}
\hline Category & Term & Gene count & $P$-value & FDR \\
\hline Annotation cluster 14 & Enrichment score: 1.73 & & & \\
\hline GOTERM_BP_FAT & Neuron projection morphogenesis & 9 & $6.50 \mathrm{E}-03$ & I.70E-0I \\
\hline GOTERM_BP_FAT & Cell projection morphogenesis & 9 & I.40E-02 & $2.20 \mathrm{E}-0 \mathrm{I}$ \\
\hline GOTERM_BP_FAT & Cell part morphogenesis & 9 & I.80E-02 & $2.40 \mathrm{E}-0 \mathrm{I}$ \\
\hline GOTERM_BP_FAT & Neuron projection development & 9 & I.80E-02 & $2.40 \mathrm{E}-0 \mathrm{I}$ \\
\hline GOTERM_BP_FAT & Neuron development & 9 & $7.40 \mathrm{E}-02$ & $4.90 \mathrm{E}-0 \mathrm{I}$ \\
\hline Annotation cluster 15 & Enrichment score: 1.49 & & & \\
\hline INTERPRO & Homeobox, conserved site & 8 & $2.40 \mathrm{E}-02$ & $7.40 \mathrm{E}-0 \mathrm{I}$ \\
\hline INTERPRO & Homeobox & 8 & $2.50 \mathrm{E}-02$ & $7.20 \mathrm{E}-0 \mathrm{I}$ \\
\hline SMART & $\mathrm{HOX}$ & 8 & $5.50 \mathrm{E}-02$ & $7.30 \mathrm{E}-0 \mathrm{I}$ \\
\hline Annotation cluster 16 & Enrichment score: I.38 & & & \\
\hline INTERPRO & Wnt superfamily & 3 & $2.20 \mathrm{E}-02$ & $7.60 \mathrm{E}-0 \mathrm{I}$ \\
\hline INTERPRO & Secreted growth factor Wnt protein & 3 & $2.20 \mathrm{E}-02$ & $7.60 \mathrm{E}-0 \mathrm{I}$ \\
\hline INTERPRO & Secreted growth factor Wnt protein, conserved site & 3 & $2.20 \mathrm{E}-02$ & $7.60 \mathrm{E}-0 \mathrm{I}$ \\
\hline PIR_SUPERFAMILY & PIRSF00 I784: int-I transforming protein & 3 & $2.60 \mathrm{E}-02$ & $7.60 \mathrm{E}-0 \mathrm{I}$ \\
\hline GOTERM_BP_FAT & Wnt receptor signaling pathway, calcium modulating pathway & 3 & $3.00 \mathrm{E}-02$ & $3.10 \mathrm{E}-0 \mathrm{I}$ \\
\hline SMART & WNTI & 3 & 3.10E-02 & $9.70 \mathrm{E}-0 \mathrm{I}$ \\
\hline KEGG_PATHWAY & Basal cell carcinoma & 3 & I.90E-0| & $6.00 \mathrm{E}-0 \mathrm{I}$ \\
\hline KEGG_PATHWAY & Hedgehog signaling pathway & 3 & $2.00 \mathrm{E}-0 \mathrm{I}$ & $6.00 \mathrm{E}-0 \mathrm{I}$ \\
\hline Annotation cluster 17 & Enrichment score: 1.26 & & & \\
\hline GOTERM_BP_FAT & Positive regulation of apoptosis & II & $5.30 \mathrm{E}-02$ & $4.30 \mathrm{E}-0 \mathrm{I}$ \\
\hline GOTERM_BP_FAT & Positive regulation of programmed cell death & 11 & $5.50 \mathrm{E}-02$ & $4.40 \mathrm{E}-0 \mathrm{I}$ \\
\hline GOTERM_BP_FAT & Positive regulation of cell death & 11 & $5.60 \mathrm{E}-02$ & $4.40 \mathrm{E}-0 \mathrm{I}$ \\
\hline Annotation cluster 18 & Enrichment Score: I.22 & & & \\
\hline UP_SEQ_FEATURE & Domain: F-box & 4 & $5.30 \mathrm{E}-02$ & $9.50 \mathrm{E}-0 \mathrm{I}$ \\
\hline INTERPRO & Cyclin-like F-box & 4 & $5.40 \mathrm{E}-02$ & $8.70 \mathrm{E}-0 \mathrm{I}$ \\
\hline SMART & FBOX & 4 & $7.90 \mathrm{E}-02$ & $8.00 \mathrm{E}-0 \mathrm{I}$ \\
\hline Annotation cluster 19 & Enrichment score: 1.05 & & & \\
\hline BIOCARTA & Cadmium induces DNA synthesis and proliferation in macrophages & 3 & $3.20 \mathrm{E}-02$ & $7.40 \mathrm{E}-0 \mathrm{I}$ \\
\hline BIOCARTA & IL-3 signaling pathway & 3 & $3.70 \mathrm{E}-02$ & $6.90 \mathrm{E}-0 \mathrm{I}$ \\
\hline BIOCARTA & NGF pathway & 3 & 5.30E-02 & $7.40 \mathrm{E}-0 \mathrm{I}$ \\
\hline BIOCARTA & EPO signaling pathway & 3 & $5.90 \mathrm{E}-02$ & $7.10 \mathrm{E}-01$ \\
\hline BIOCARTA & Inhibition of cellular proliferation by Gleevec & 3 & $6.50 \mathrm{E}-02$ & $6.90 \mathrm{E}-0 \mathrm{I}$ \\
\hline BIOCARTA & TPO signaling pathway & 3 & 7.10E-02 & $6.80 \mathrm{E}-0 \mathrm{I}$ \\
\hline BIOCARTA & Signaling pathway from G-protein families & 3 & $7.80 \mathrm{E}-02$ & $6.70 \mathrm{E}-0 \mathrm{I}$ \\
\hline BIOCARTA & IL-2 signaling pathway & 3 & $7.80 \mathrm{E}-02$ & $6.70 \mathrm{E}-0 \mathrm{I}$ \\
\hline BIOCARTA & PDGF signaling pathway & 3 & I.00E-0I & $7.40 \mathrm{E}-0 \mathrm{I}$ \\
\hline BIOCARTA & BCR signaling pathway & 3 & I.IOE-0I & $7.40 \mathrm{E}-0 \mathrm{I}$ \\
\hline BIOCARTA & EGF signaling pathway & 3 & I.IOE-0I & $7.40 \mathrm{E}-0 \mathrm{I}$ \\
\hline BIOCARTA & Fc epsilon receptor I signaling in mast cells & 3 & I.40E-0I & $7.70 \mathrm{E}-0 \mathrm{I}$ \\
\hline BIOCARTA & T-cell receptor signaling pathway & 3 & I.70E-0I & $7.90 \mathrm{E}-0 \mathrm{I}$ \\
\hline BIOCARTA & MAP kinase signaling pathway & 3 & $5.40 \mathrm{E}-0 \mathrm{I}$ & $1.00 \mathrm{E}+00$ \\
\hline Annotation cluster 20 & Enrichment score: $\mathrm{I} .03$ & & & \\
\hline UP_SEQ_FEATURE & Short sequence motif: $\mathrm{BH} 3$ & 3 & I.70E-02 & $7.10 \mathrm{E}-01$ \\
\hline INTERPRO & Apoptosis regulator $\mathrm{Bcl}-2, \mathrm{BH}$ & 3 & $2.90 \mathrm{E}-02$ & $7.30 \mathrm{E}-0 \mathrm{I}$ \\
\hline SMART & $\mathrm{Bcl}$ & 3 & 4.00E-02 & $7.90 \mathrm{E}-0 \mathrm{I}$ \\
\hline GOTERM_CC_FAT & Mitochondrial outer membrane & 3 & $2.80 \mathrm{E}-0 \mathrm{I}$ & $9.50 \mathrm{E}-0 \mathrm{I}$ \\
\hline GOTERM_CC_FAT & Organelle outer membrane & 3 & $3.40 \mathrm{E}-0 \mathrm{I}$ & $9.60 \mathrm{E}-0 \mathrm{I}$ \\
\hline GOTERM_CC_FAT & Outer membrane & 3 & $3.60 \mathrm{E}-0 \mathrm{I}$ & $9.60 \mathrm{E}-0 \mathrm{I}$ \\
\hline
\end{tabular}

Abbreviations: Bcl, B-cell lymphoma; BCR, B-cell receptor; DAVID, Database for Annotation, Visualization and Integrated Discovery; EGF, epidermal growth factor; EPO, erythropoietin; FDR, false discovery rate; IGF, insulin-like growth factor; IL, interleukin; NGF, nerve growth factor; PDGF, platelet-derived growth factor; TPO, thrombopoietin.

of cellular biosynthetic process, negative regulation of biosynthetic process, regulation of phosphorylation, regulation of phosphate metabolic process, lung development, respiratory tube development, positive regulation of transcription, positive regulation of gene expression, mesenchymal cell differentiation and development, negative regulation of apoptosis and programmed cell death, the insulin-like growth factor (IGF)-1 signaling pathway, interleukin (IL)-6 signaling pathway, insulin signaling pathway, Ubl conjugation pathway, modification-dependent macromolecule catabolic process, and modification-dependent protein catabolic process. 
Table 10 The KEGG pathways by DAVID for the target list of hsa-miR-I8Ia-5p based on miRTarBase

\begin{tabular}{lllll}
\hline Signaling pathway & $\begin{array}{l}\text { Gene } \\
\text { count }\end{array}$ & $\%$ & P-value & FDR \\
& 14 & 6.2 & $8.70 \mathrm{E}-04$ & $8.20 \mathrm{E}-02$ \\
\hline Pathways in cancer & 9 & 4 & $4.20 \mathrm{E}-02$ & $3.70 \mathrm{E}-0 \mathrm{I}$ \\
MAPK signaling pathway & 7 & 3.1 & $3.20 \mathrm{E}-03$ & $1.40 \mathrm{E}-0 \mathrm{I}$ \\
Melanogenesis & 5 & 2.2 & $2.40 \mathrm{E}-02$ & $3.80 \mathrm{E}-0 \mathrm{I}$ \\
Chronic myeloid leukemia & 5 & 2.2 & $3.50 \mathrm{E}-02$ & $3.90 \mathrm{E}-0 \mathrm{I}$ \\
Small cell lung cancer & 5 & 2.2 & $4.20 \mathrm{E}-02$ & $4.10 \mathrm{E}-0 \mathrm{I}$ \\
Prostate cancer & 4 & $\mathrm{I} .8$ & $5.60 \mathrm{E}-03$ & $1.70 \mathrm{E}-0 \mathrm{I}$ \\
Dorsoventral axis formation & 4 & 1.8 & $8.60 \mathrm{E}-03$ & $1.90 \mathrm{E}-0 \mathrm{I}$ \\
Thyroid cancer & 4 & 1.8 & $3.10 \mathrm{E}-02$ & $4.10 \mathrm{E}-0 \mathrm{I}$ \\
Notch signaling pathway & 4 & 1.8 & $8.10 \mathrm{E}-02$ & $5.60 \mathrm{E}-0 \mathrm{I}$ \\
Long-term depression & 4 & $\mathrm{I} .8$ & $8.40 \mathrm{E}-02$ & $5.40 \mathrm{E}-0 \mathrm{I}$ \\
Renal cell carcinoma & 4 & $\mathrm{I} .8$ & $9.80 \mathrm{E}-02$ & $5.40 \mathrm{E}-0 \mathrm{I}$ \\
B-cell receptor signaling pathway & 4 & 1.8 & $9.80 \mathrm{E}-02$ & $5.40 \mathrm{E}-0 \mathrm{I}$ \\
VEGF signaling pathway & 3 & $\mathrm{I} .3$ & $9.40 \mathrm{E}-02$ & $5.50 \mathrm{E}-0 \mathrm{I}$ \\
Prion diseases & &
\end{tabular}

Abbreviations: DAVID, Database for Annotation, Visualization and Integrated Discovery; FDR, false discovery rate; KEGG, Kyoto Encyclopedia of Genes and Genomes; VEGF, vascular endothelial growth factor.

Furthermore, our DAVID analysis revealed that there were 14 KEGG pathways significantly enriched in the target list of hsa-miR-181a-5p, based on miRTarBase (Table 10). These pathways included pathways in cancer pathways (Figure 5), the MAPK signaling pathway (Figure 6), melanogenesis, chronic myeloid leukemia, small cell lung cancer, prostate cancer, dorsoventral axis formation, thyroid cancer, the Notch signaling pathway (Figure 7), long-term depression, renal cell carcinoma, the B cell receptor signaling pathway, vascular endothelial growth factor (VEGF) signaling pathway (Figure 8), and prion diseases.

\section{Combined validated targets of hsa-miR- I8Ia-5p, based on both TarBase 6.0 and miRTarBase 4.0}

When we combined the target lists of hsa-miR-181a-5p with experimental evidence from both TarBase 6.0 and miRTarBase 4.0, there were 313 validated targets for hsamiR-181a-5p (Table 11). As shown in Table 12, our DAVID analysis showed that there were 26 functional clusters that were identified to be enriched with an enrichment score $>1.0$ in the combined target list of hsa-miR-181a-5p, based on both TarBase 6.0 and miRTarBase 4.0. The functions of these clusters involved response to hormone stimulus, response to endogenous stimulus, response to organic substance, negative regulation of apoptosis, negative and positive regulation of programmed cell death, negative and positive regulation of cell death, the DNA damage checkpoint, DNA integrity checkpoint, DNA damage response and signal transduction, the cell cycle checkpoint, response to DNA damage stimulus, bladder cancer, endometrial cancer (Figure 9), non-small-cell lung cancer, acute myeloid leukemia, glioma, melanoma, developmental growth, cell fate commitment, tissue morphogenesis, positive regulation of macromolecule biosynthetic process, positive regulation of cellular biosynthetic process, positive regulation of biosynthetic process, regulation of phosphorylation, regulation of phosphate metabolic process, regulation of phosphorus metabolic process, positive regulation of transcription, positive regulation of gene expression, positive regulation of protein kinase activity, positive regulation of kinase activity, positive regulation of transferase activity, regulation of protein kinase activity, regulation of kinase activity, positive regulation of cellular protein metabolic process, positive regulation of protein metabolic process, branching morphogenesis of a tube, positive regulation of cell development, morphogenesis of a branching structure, tube morphogenesis, regulation of cell development, neuron projection morphogenesis, cell projection morphogenesis, neuron projection development, cell part morphogenesis, neuron development, cell morphogenesis, cell projection organization, cellular component morphogenesis, neuron differentiation, IGF-1 signaling pathway, IL6 signaling pathway, insulin signaling pathway, signaling of hepatocyte growth factor receptor, embryonic appendage morphogenesis, embryonic limb morphogenesis, limb morphogenesis, appendage morphogenesis, limb development, appendage development, embryonic morphogenesis, response to ethanol, response to metal ion, response to inorganic substance, response to drug, response to estrogen stimulus, positive regulation of protein modification process, regulation of protein modification process, protein amino acid phosphorylation, phosphorylation, phosphate metabolic process, phosphorus metabolic process, cell aging, negative regulation of neuron apoptosis, aging, actin cytoskeleton organization, actin filament-based process, membrane organization, membrane insoluble fraction, Ras protein signal transduction, long-term depression, the B cell receptor signaling pathway, VEGF signaling pathway, Fc epsilon RI signaling pathway, ErbB signaling pathway, gap junction, gonadotropin-releasing hormone (GnRH) signaling pathway, $\mathrm{T}$ cell receptor signaling pathway, insulin signaling pathway, small GTPase-mediated signal transduction, chemokine signaling pathway, regulation of actin cytoskeleton, MAPK signaling pathway, axonogenesis, cell morphogenesis involved in neuron differentiation, cell morphogenesis involved in differentiation, nucleoplasm, 


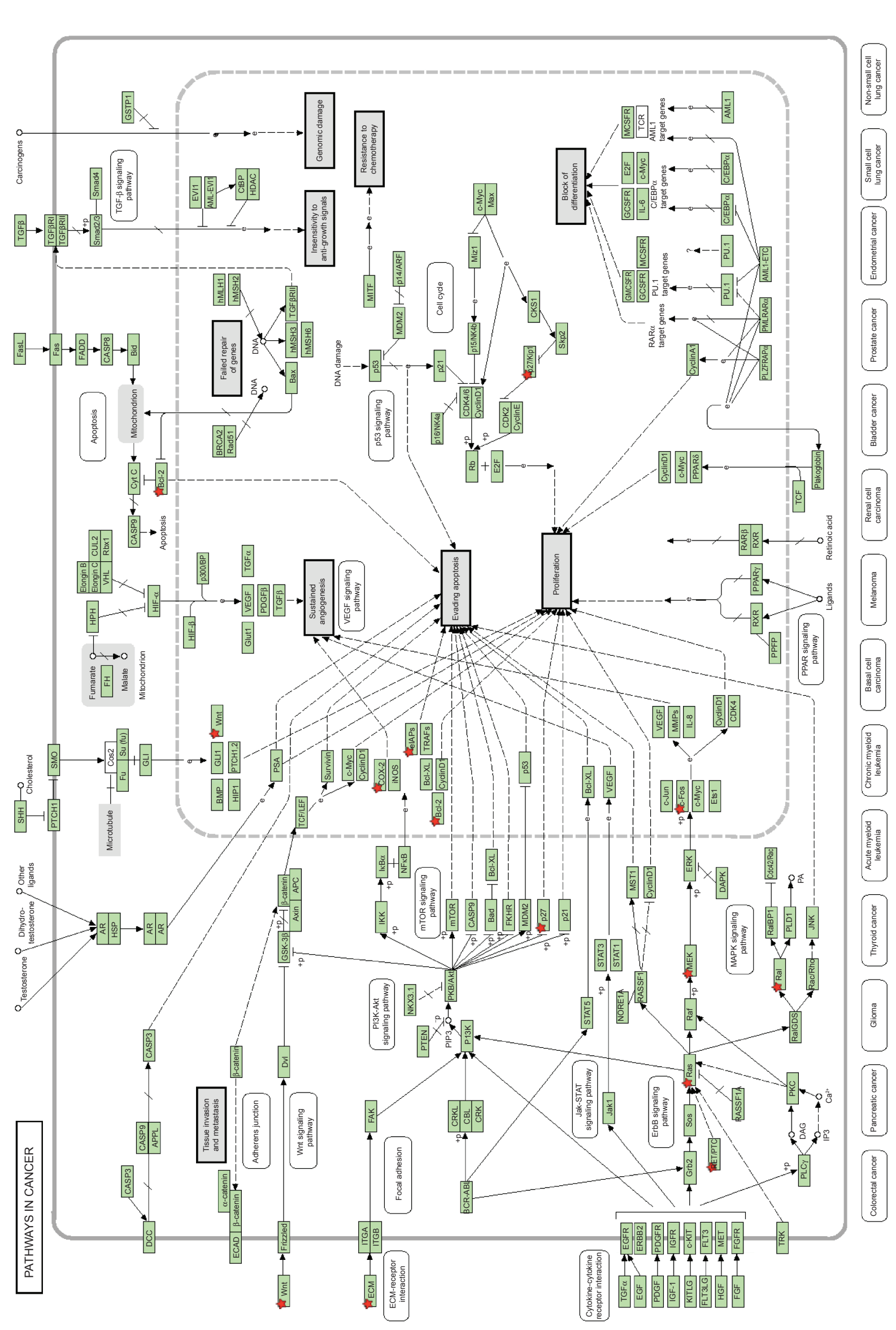

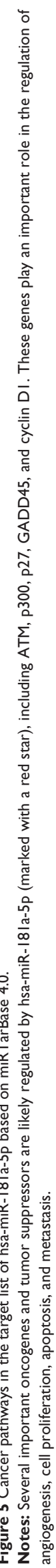




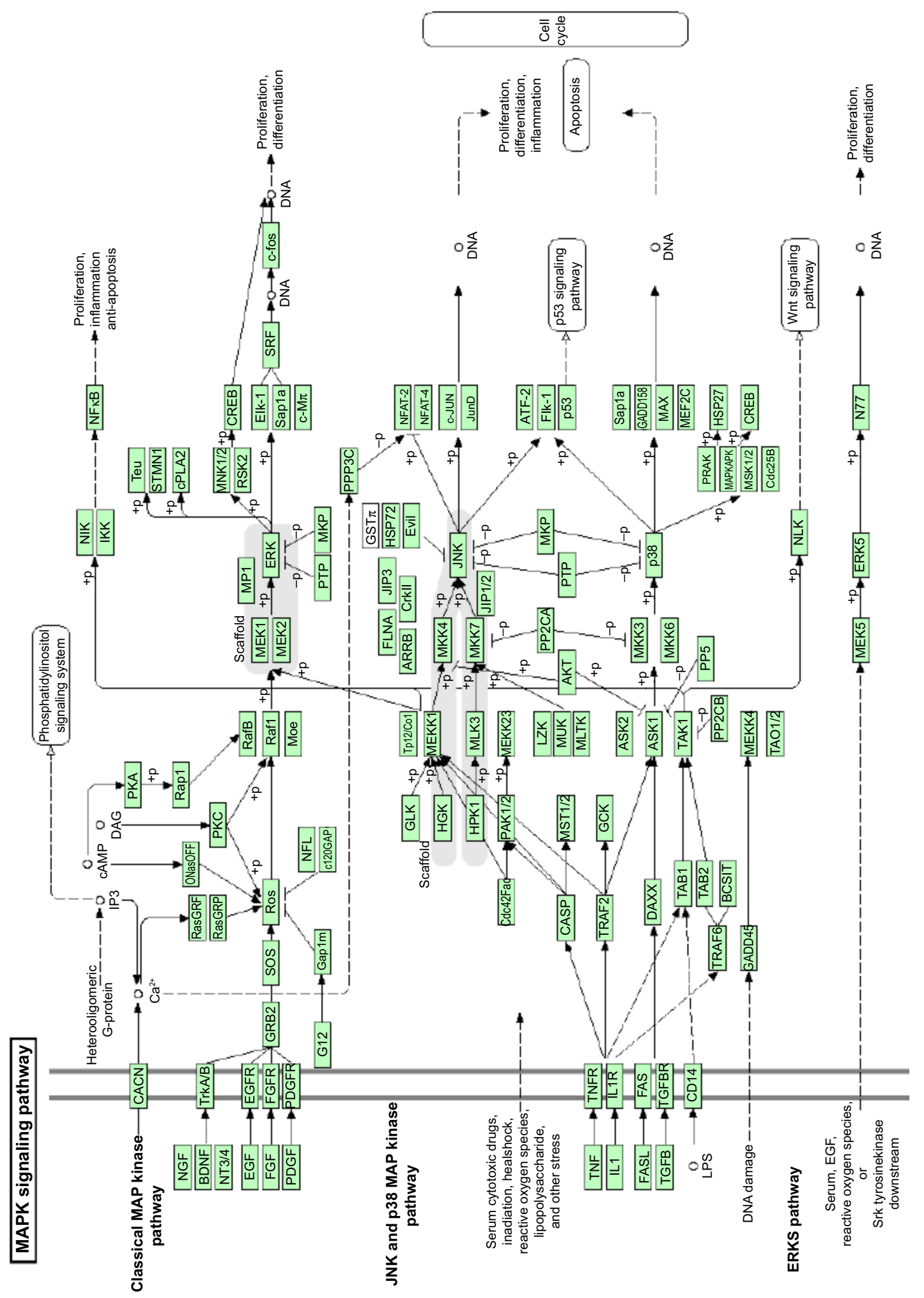

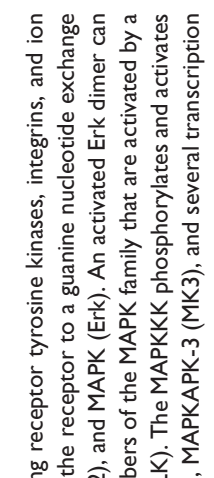

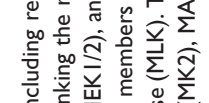

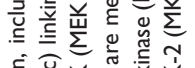

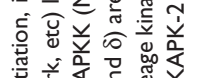

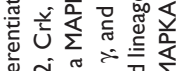

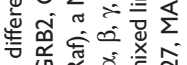

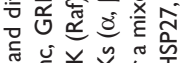

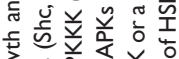

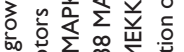

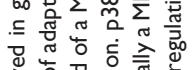

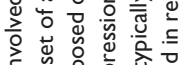

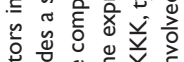

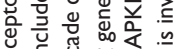

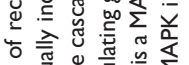

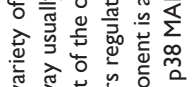

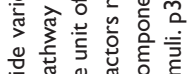

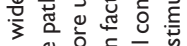

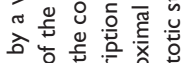

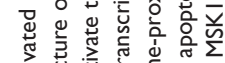

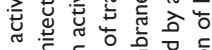

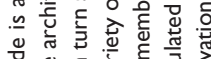

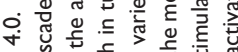

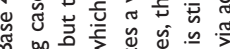

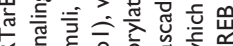

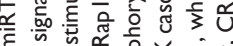

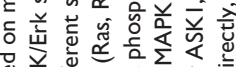

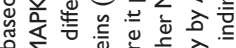

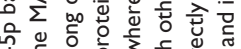

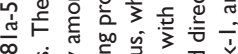

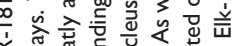

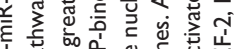

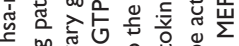

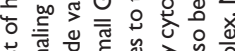

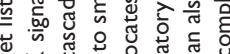

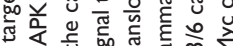

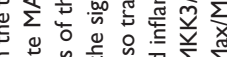

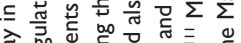

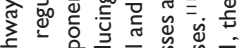

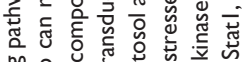

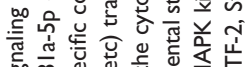

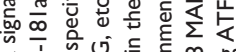

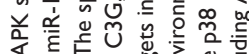

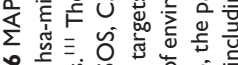

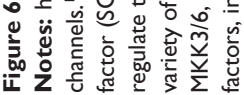


Notch signaling pathway

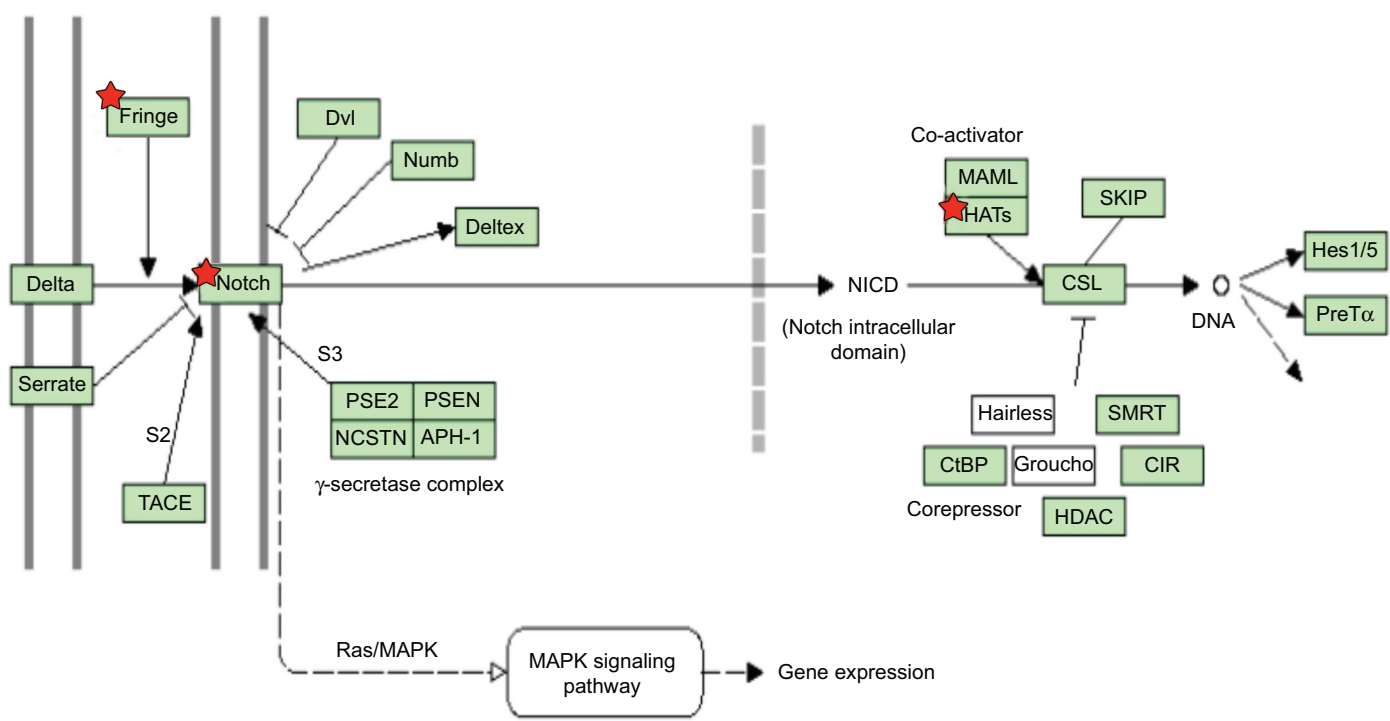

Figure 7 Notch signaling pathway in the target list of hsa-miR-I8la-5p based on miRTarBase 4.0.

Notes: hsa-miR-18la-5p can regulate the function of the Notch signaling pathway. Notch signaling is an evolutionarily conserved pathway in multicellular organisms that regulates cell fate determination during development and maintains adult tissue homeostasis. In mammalian signal-sending cells, members of the Delta-like (DLLI, DLL3 \& DLL4) and the Jagged (JAGI \& JAG2) families serve as ligands for Notch signaling receptors. ${ }^{12}$ Upon ligand binding, the NECD is cleaved away (S2 cleavage) from the TMNICD domain by TACE (TNF- $\alpha$ ADAM metalloprotease converting enzyme). The NECD remains bound to the ligand, and this complex undergoes endocytosis/recycling within the signal-sending cell in a manner dependent on ubiquitination by Mib. In the signal-receiving cell, $\gamma$-secretase (also involved in Alzheimer's disease) releases the NICD from the TM (S3 cleavage), which allows for nuclear translocation where it associates with the CSL (CBFI/Su(H)/Lag-I) transcription factor complex, resulting in subsequent activation of the canonical Notch target genes, including Myc, p2I, and the HES-family members. Abnormal expression of Notch and related proteins has been observed in $\mathrm{EC}$, and the Notch signaling pathway may play a role in the development, growth, and metastasis of EC. ${ }^{113-116}$ Targets of hsa-miR-18I a-5p are marked with a red star. Abbreviations: EC, endometrial cancer; TNF, tumor necrosis factor.

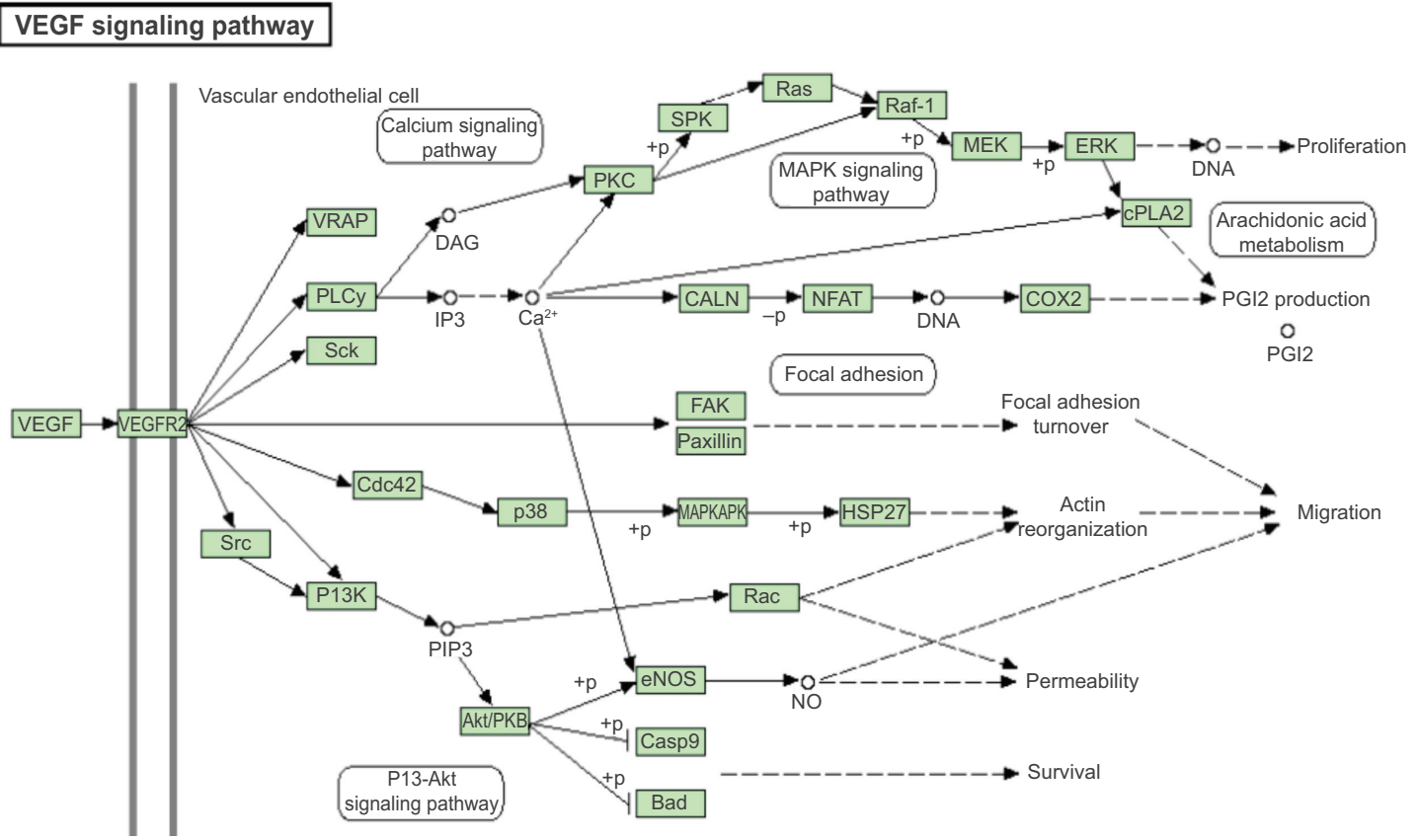

Figure 8 VEGF signaling pathway in the target list of hsa-miR-18Ia-5p based on miRTarBase 4.0.

Notes: hsa-miR-18Ia-5p can regulate the VEGF signaling pathway. VEGF is an important signaling protein involved in both vasculogenesis and angiogenesis. All members of the VEGF family stimulate cellular responses by binding to tyrosine kinase receptors (the VEGFRs) on the cell surface, causing them to dimerize and become activated through transphosphorylation. This triggers a signaling cascade that activates several signaling pathways, such as PI3K/Akt, Erk I/2, Smad, and Notch, and results in endothelial cell proliferation and migration. A number of studies have shown that VEGF and its associated proteins are aberrant in EC. ${ }^{117-122}$ These proteins represent useful targets in the treatment of EC.

Abbreviations: EC, endometrial cancer; VEGF, vascular endothelial growth factor. 


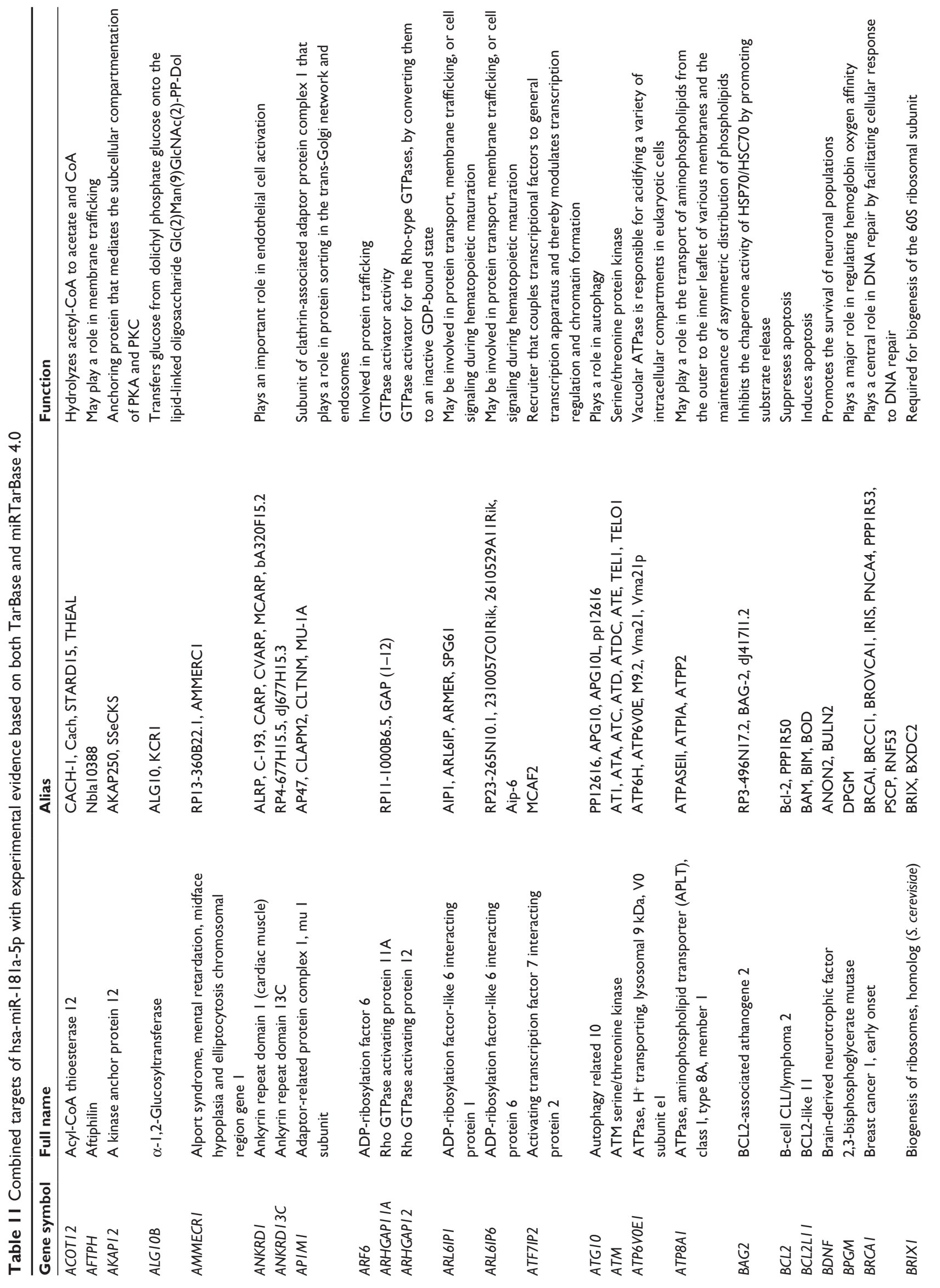



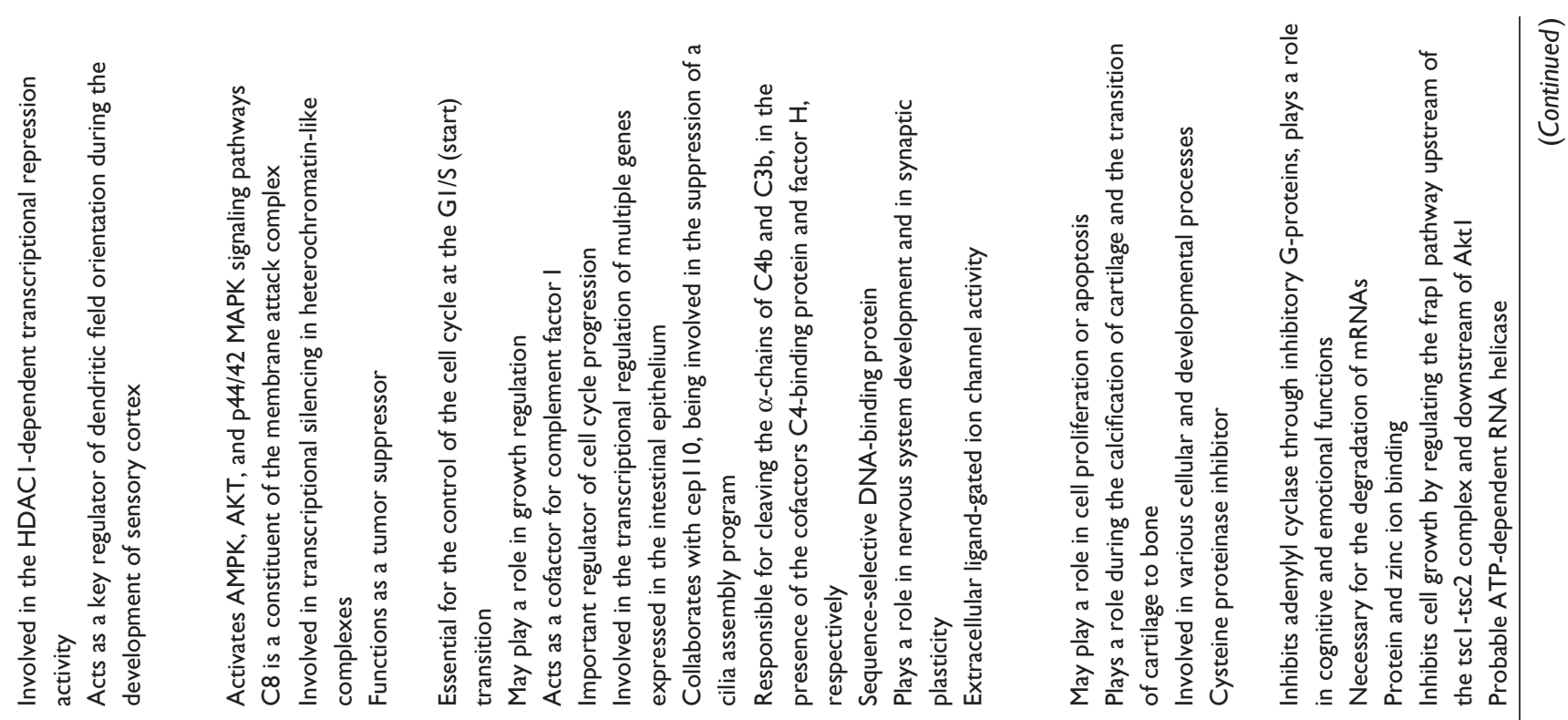

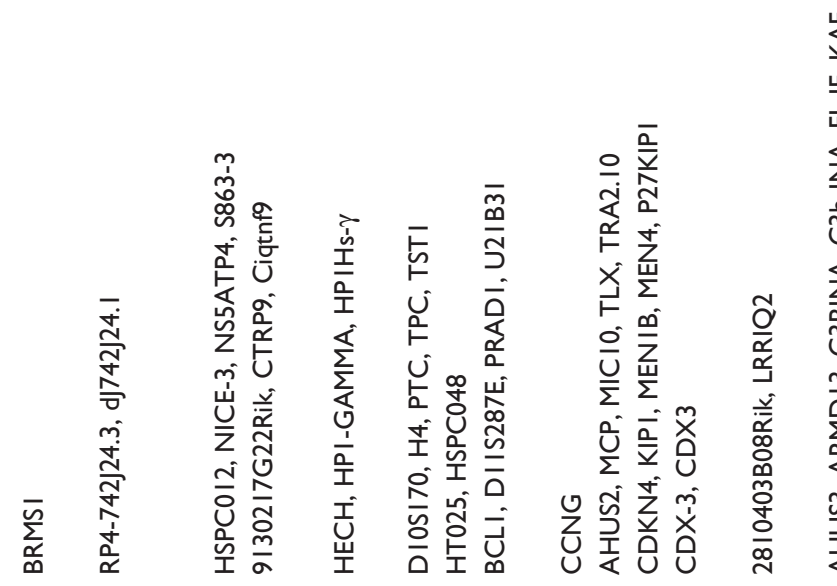
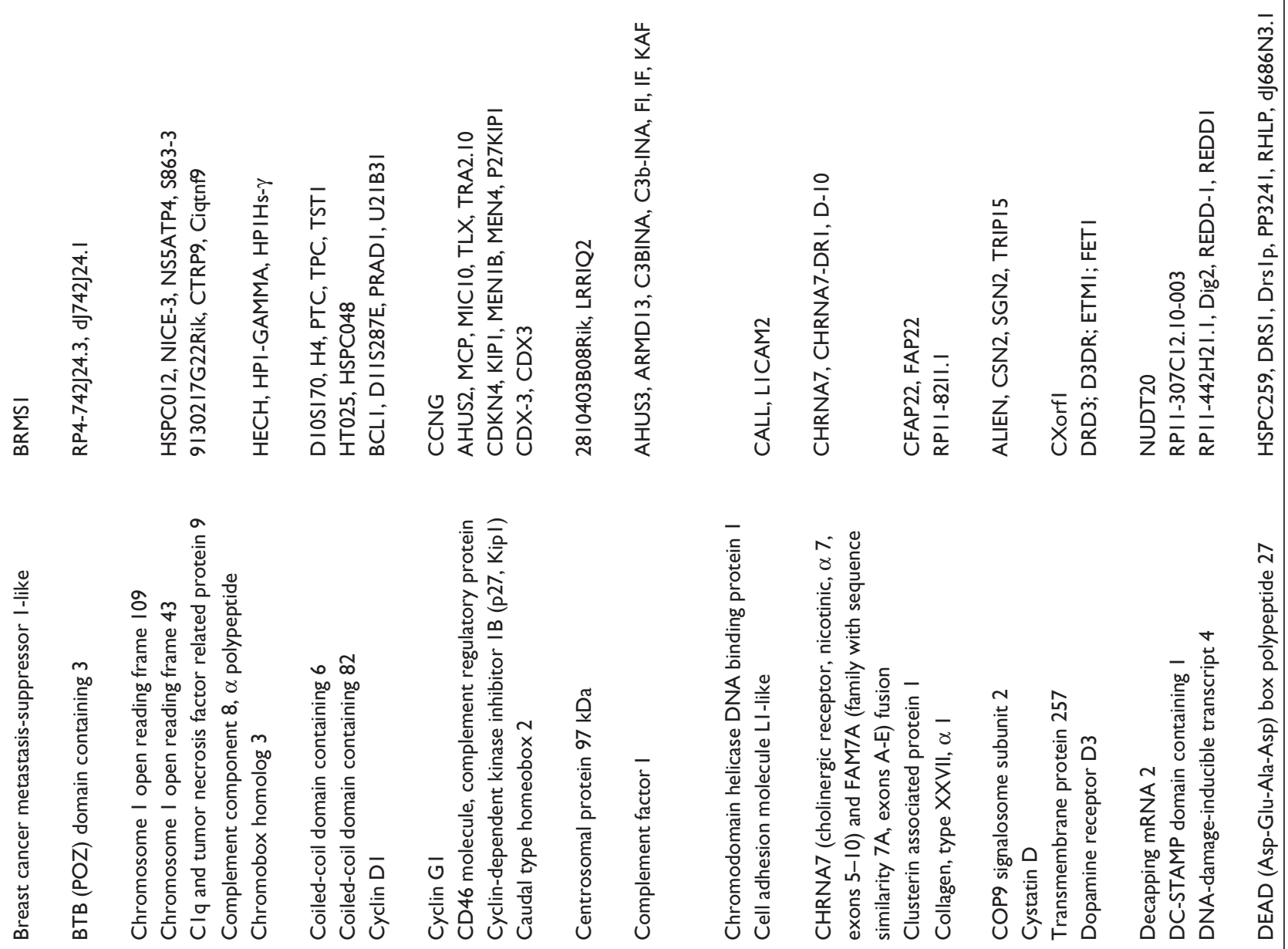

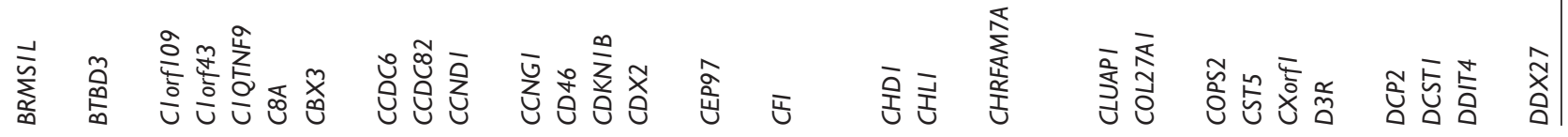



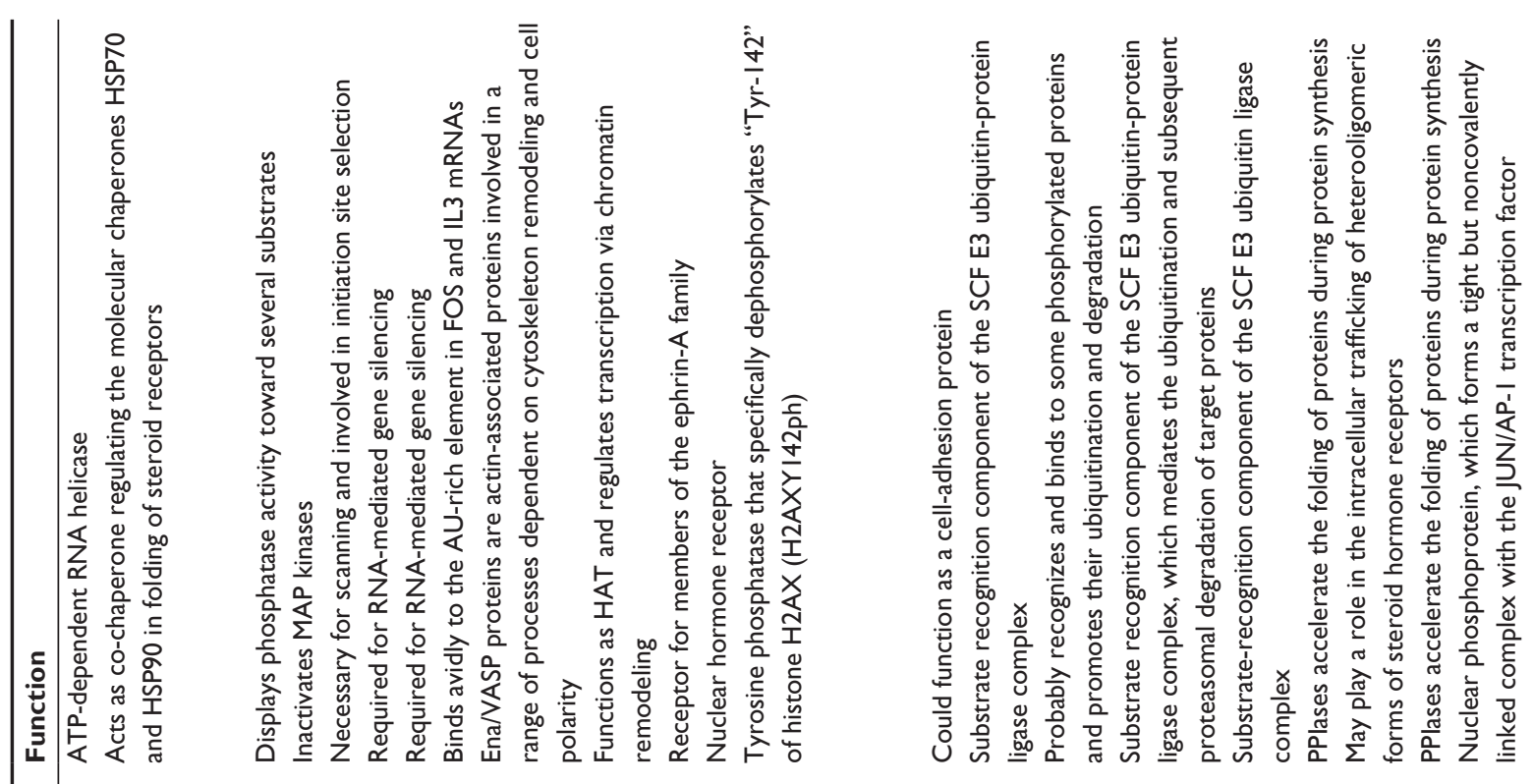

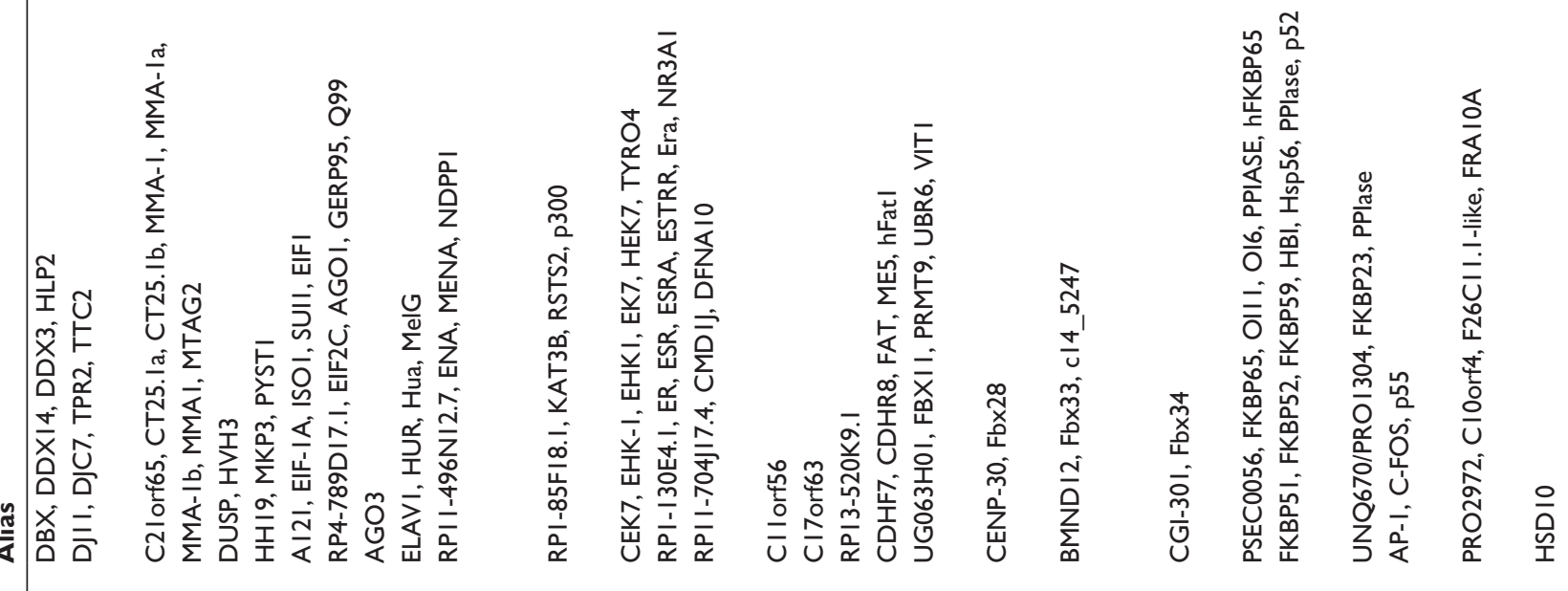
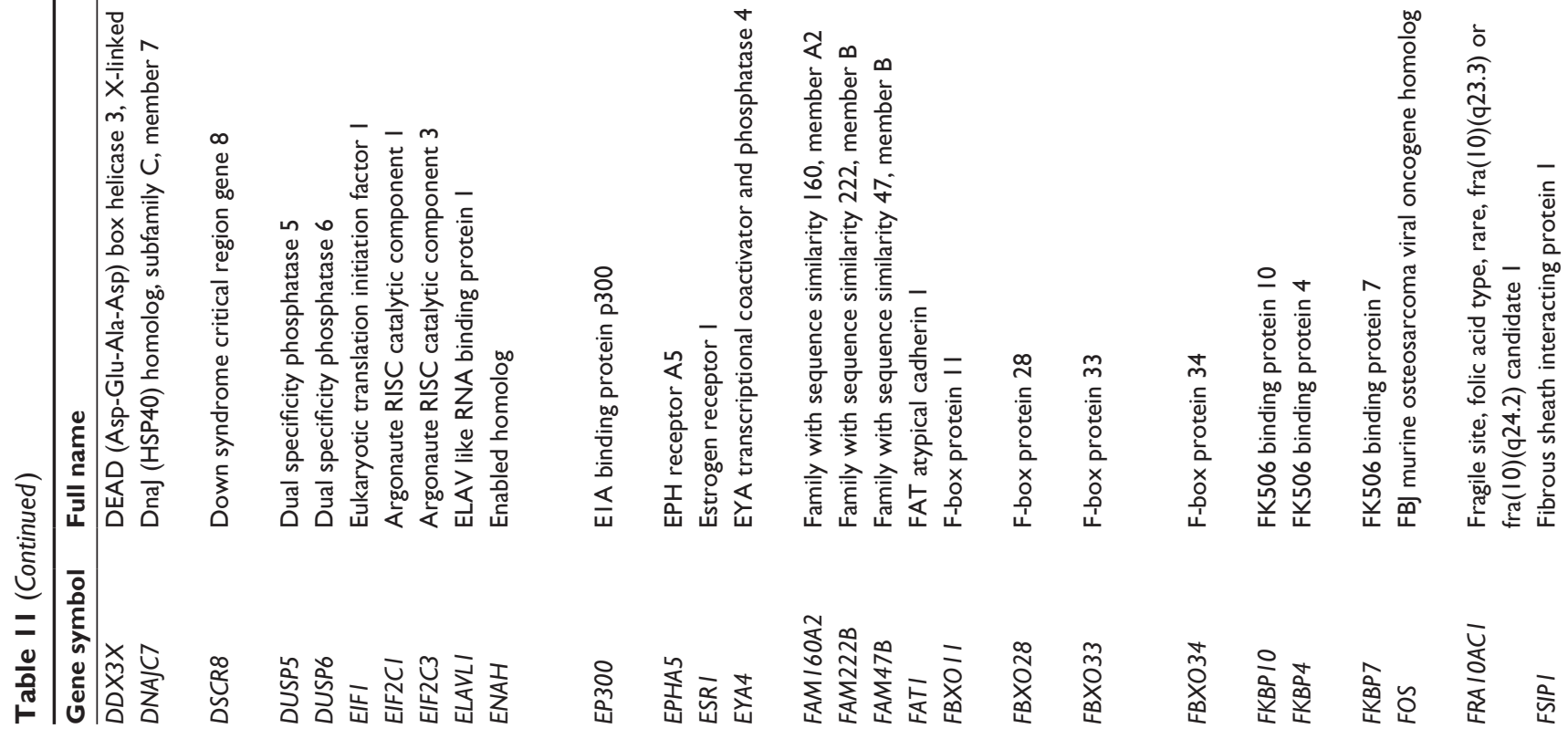

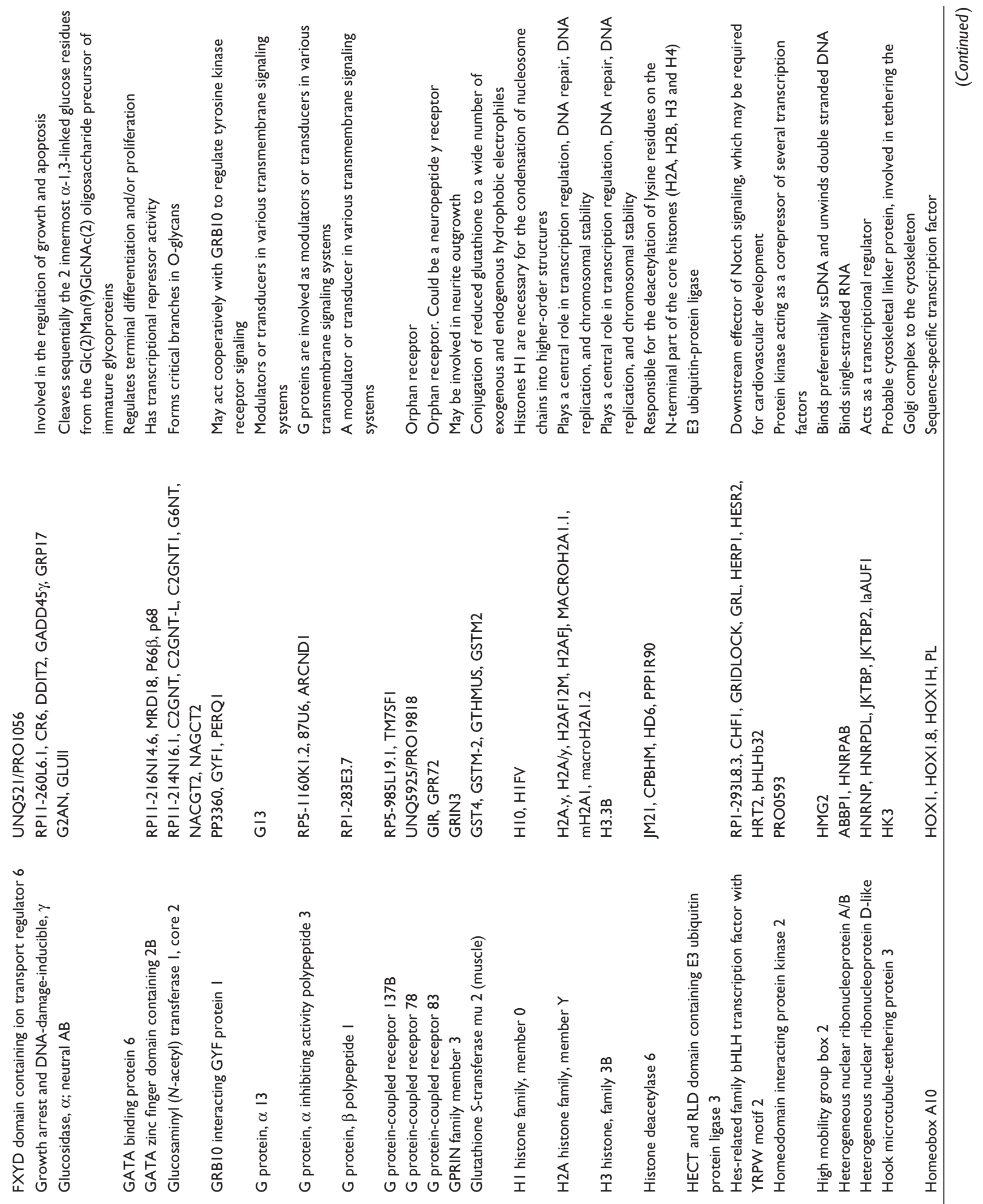

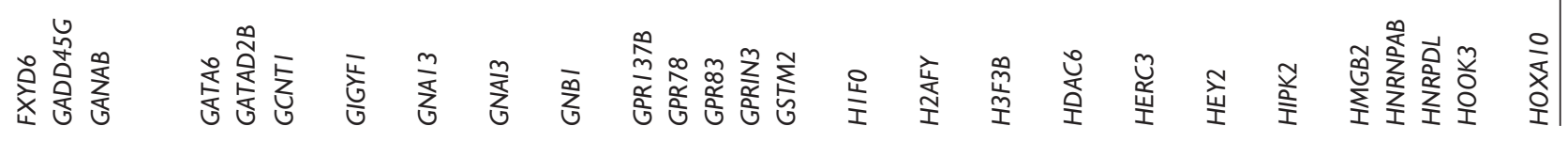



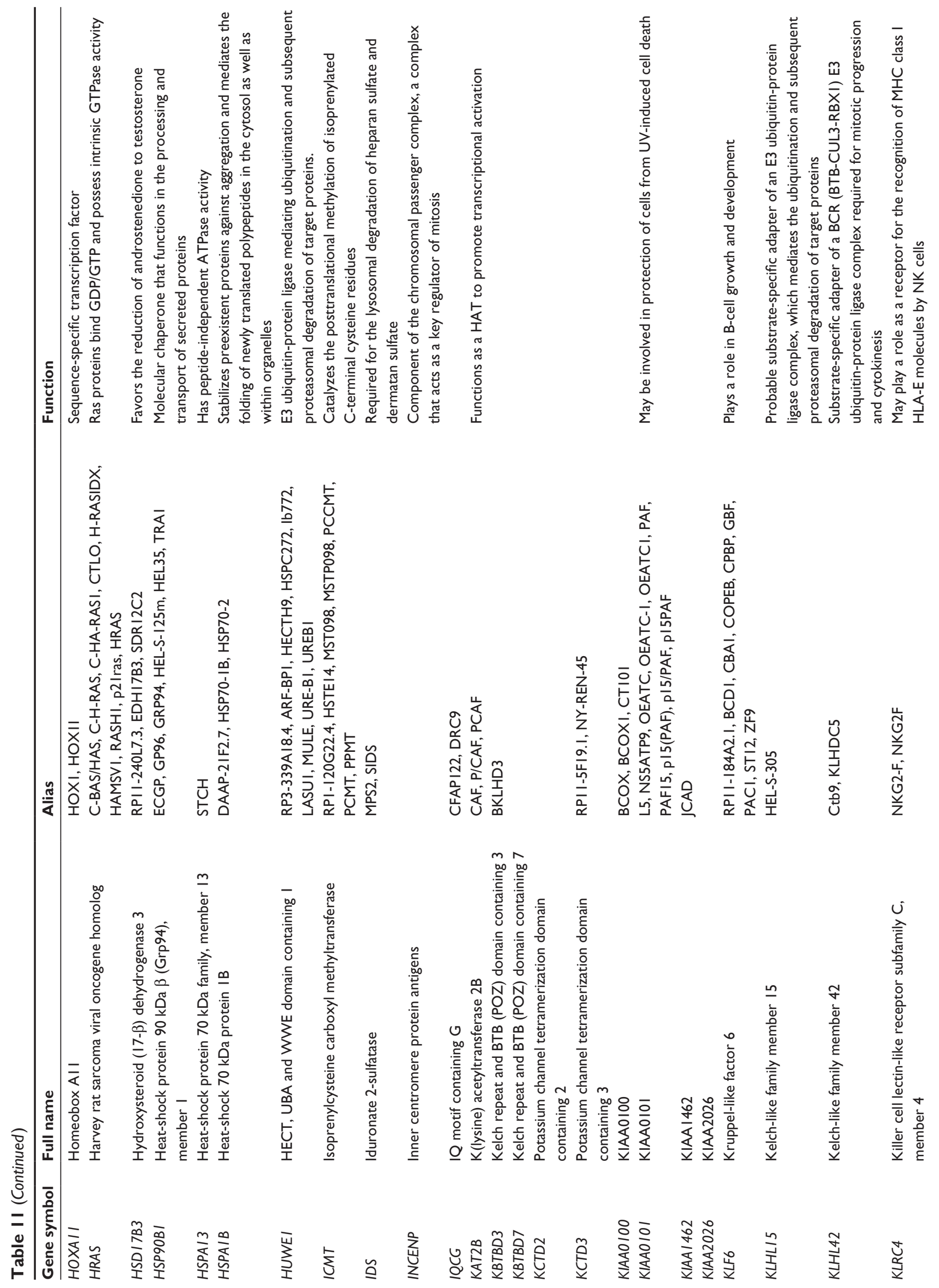

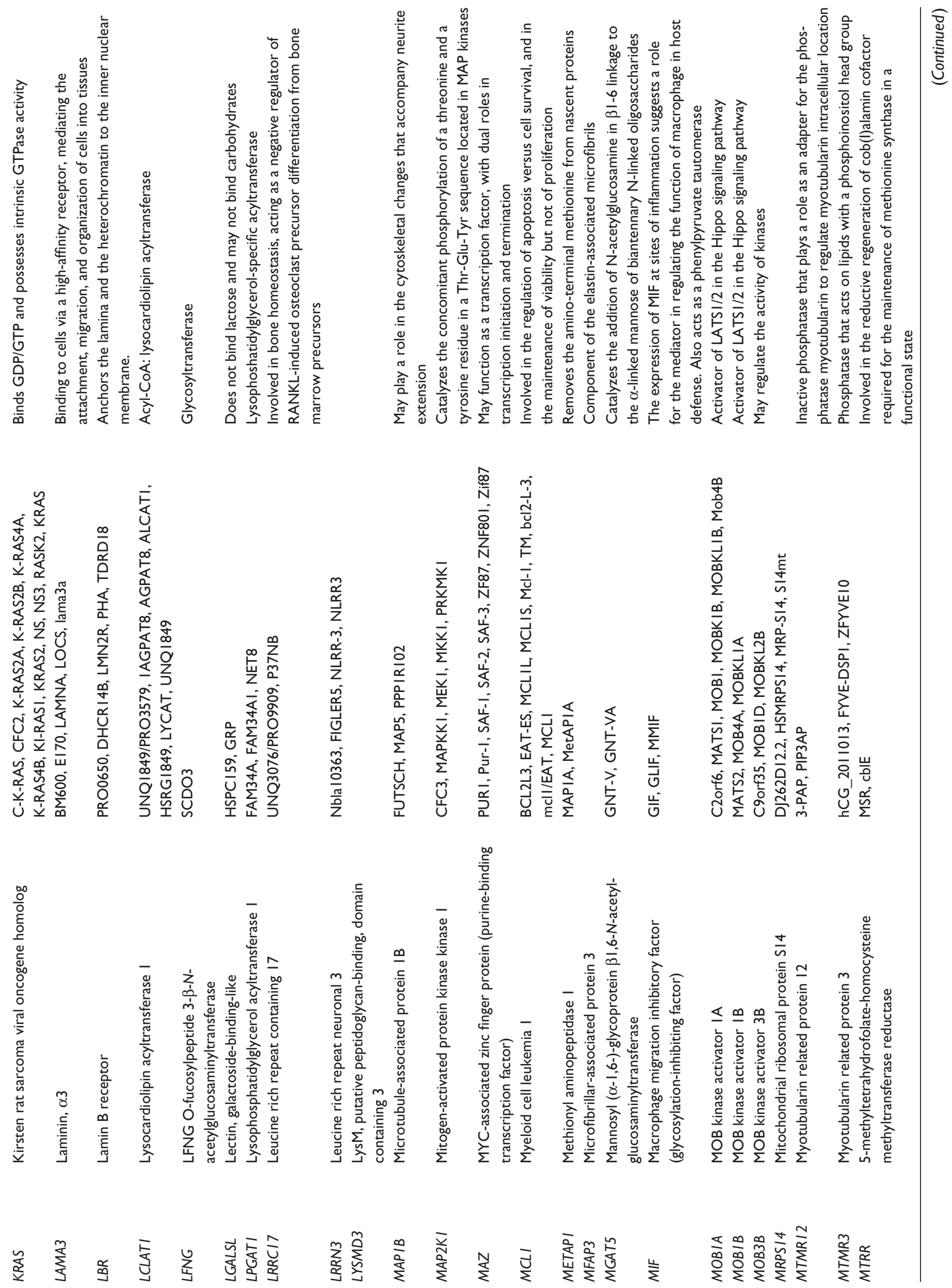

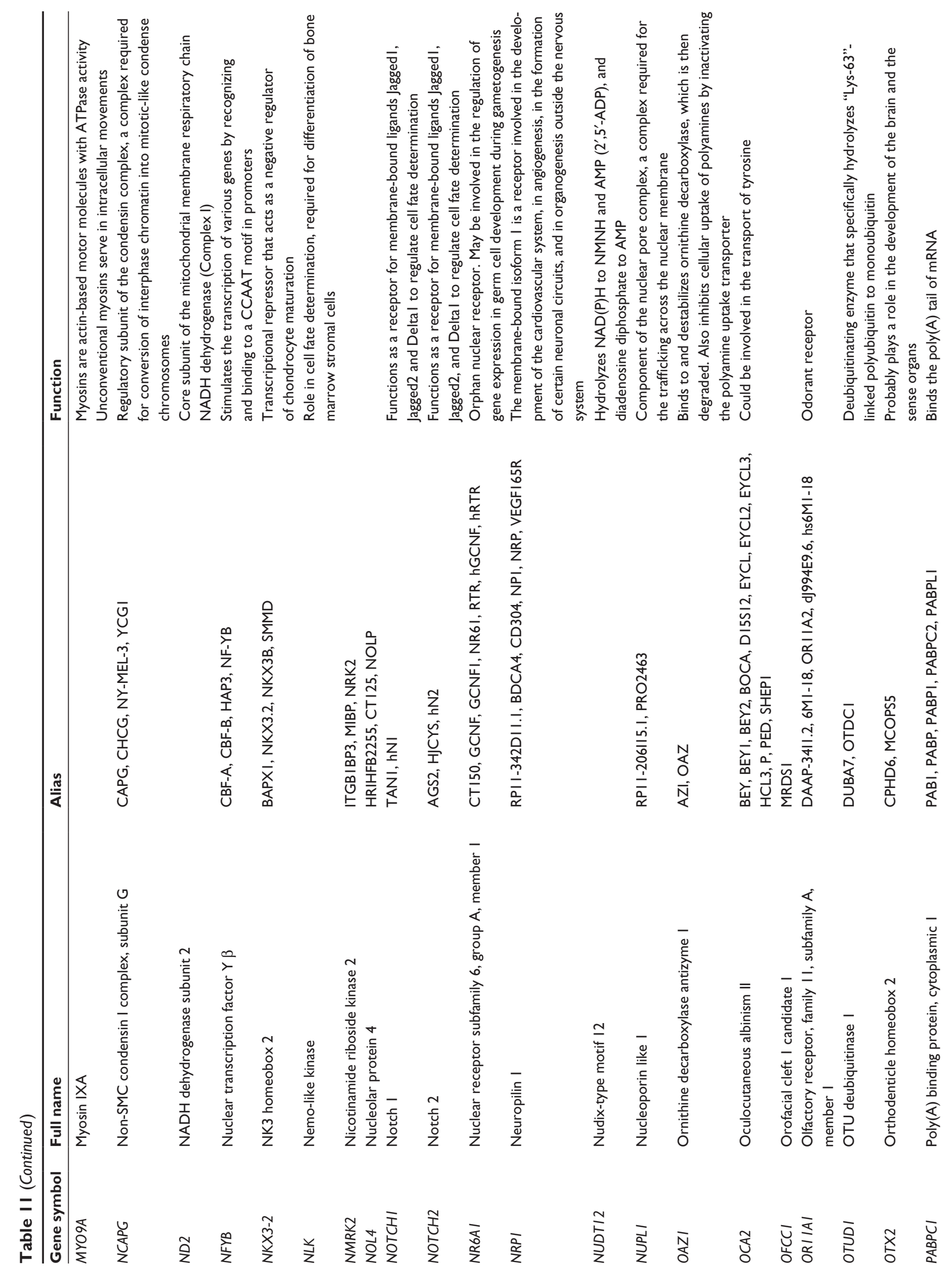

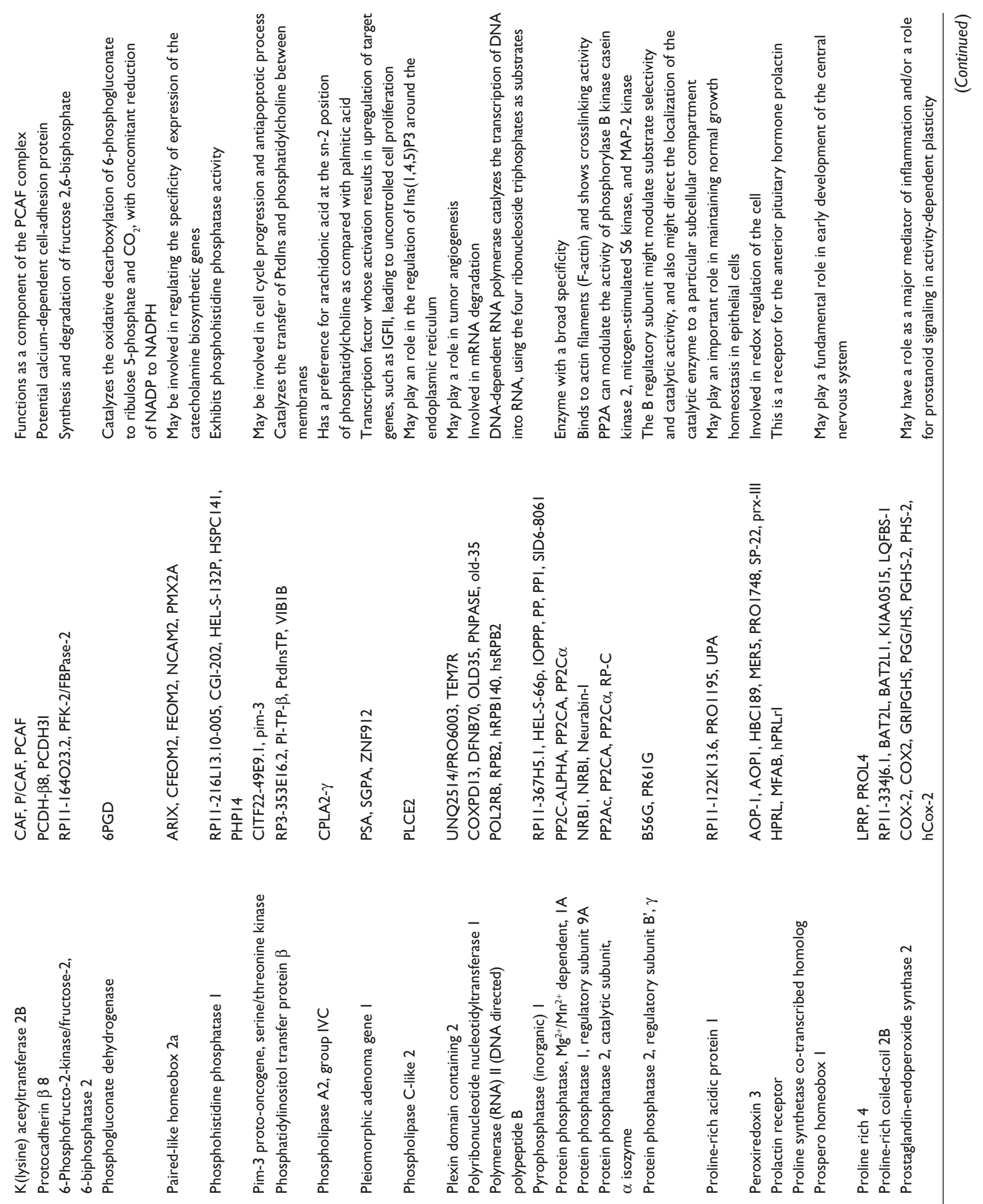

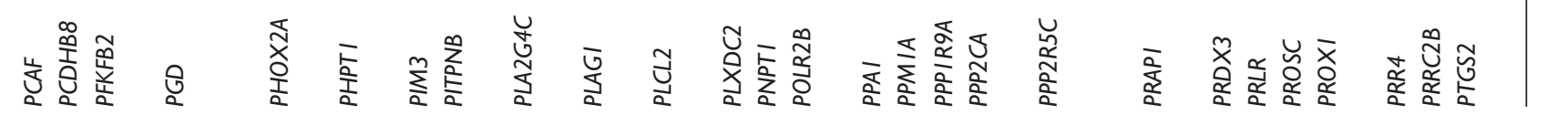



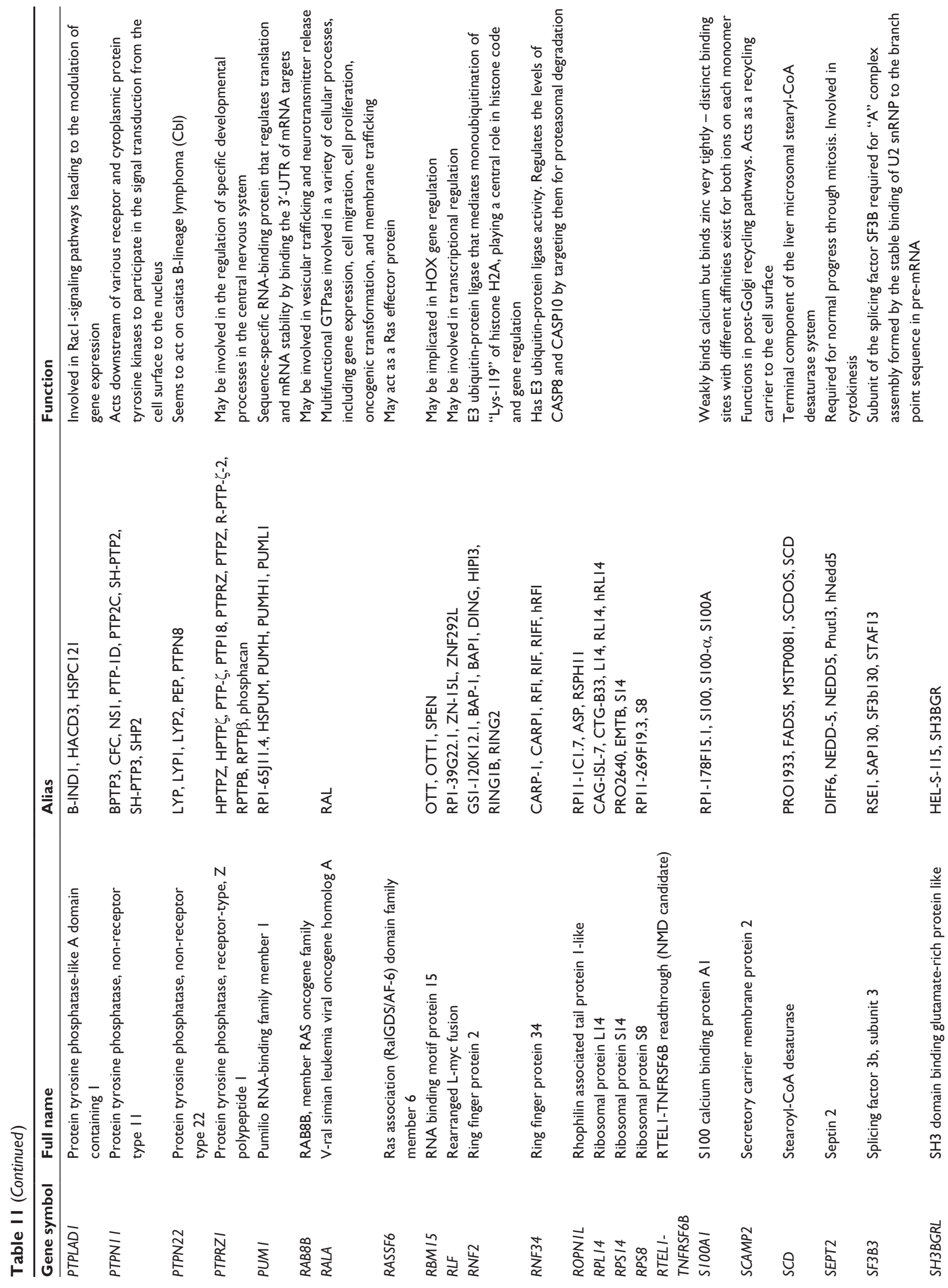

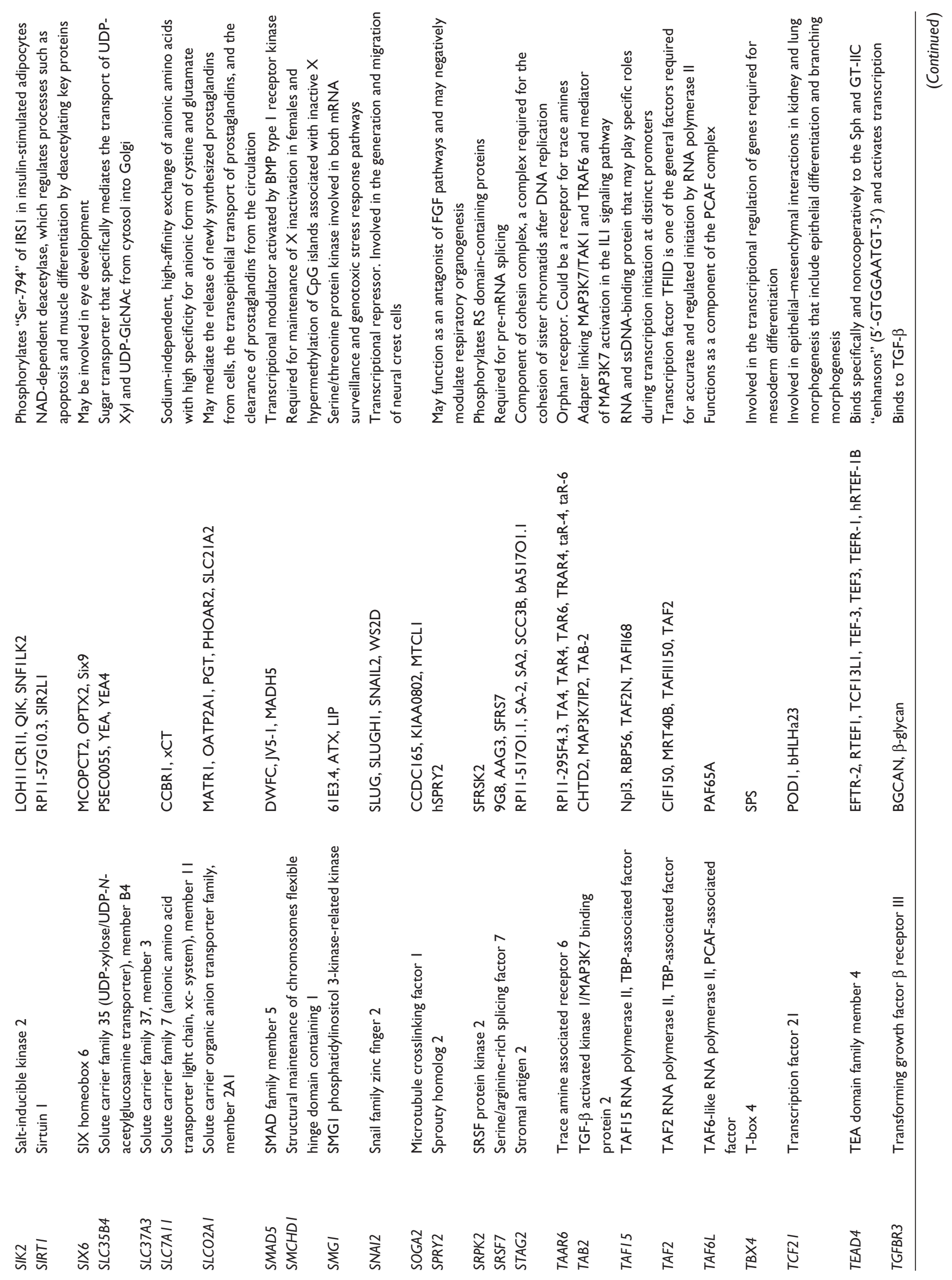

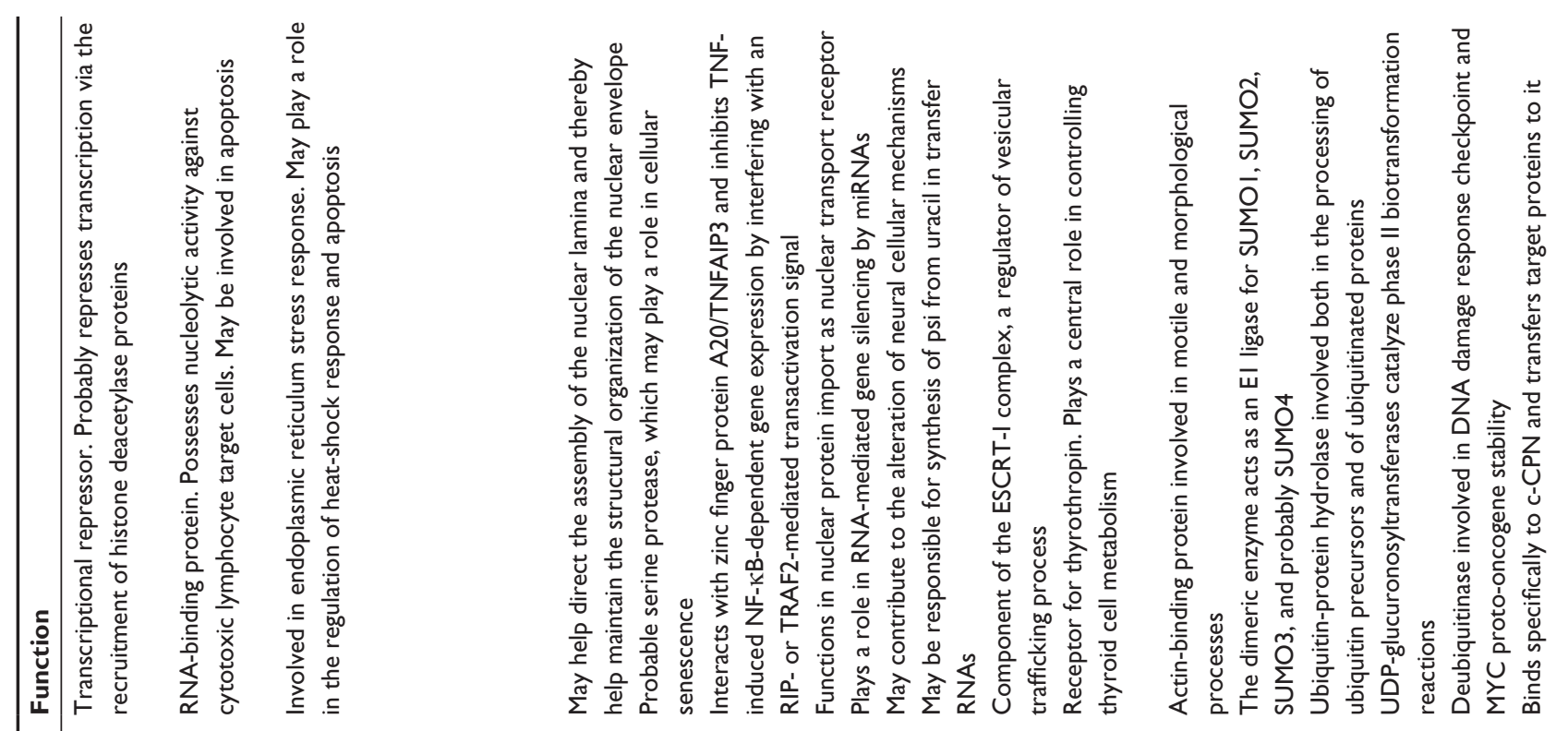

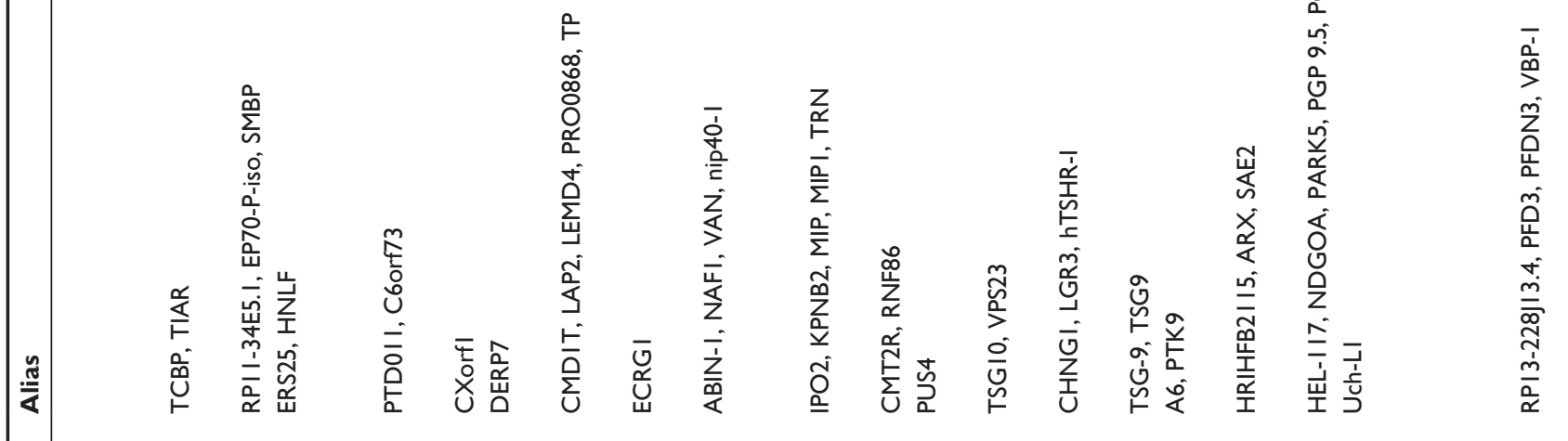

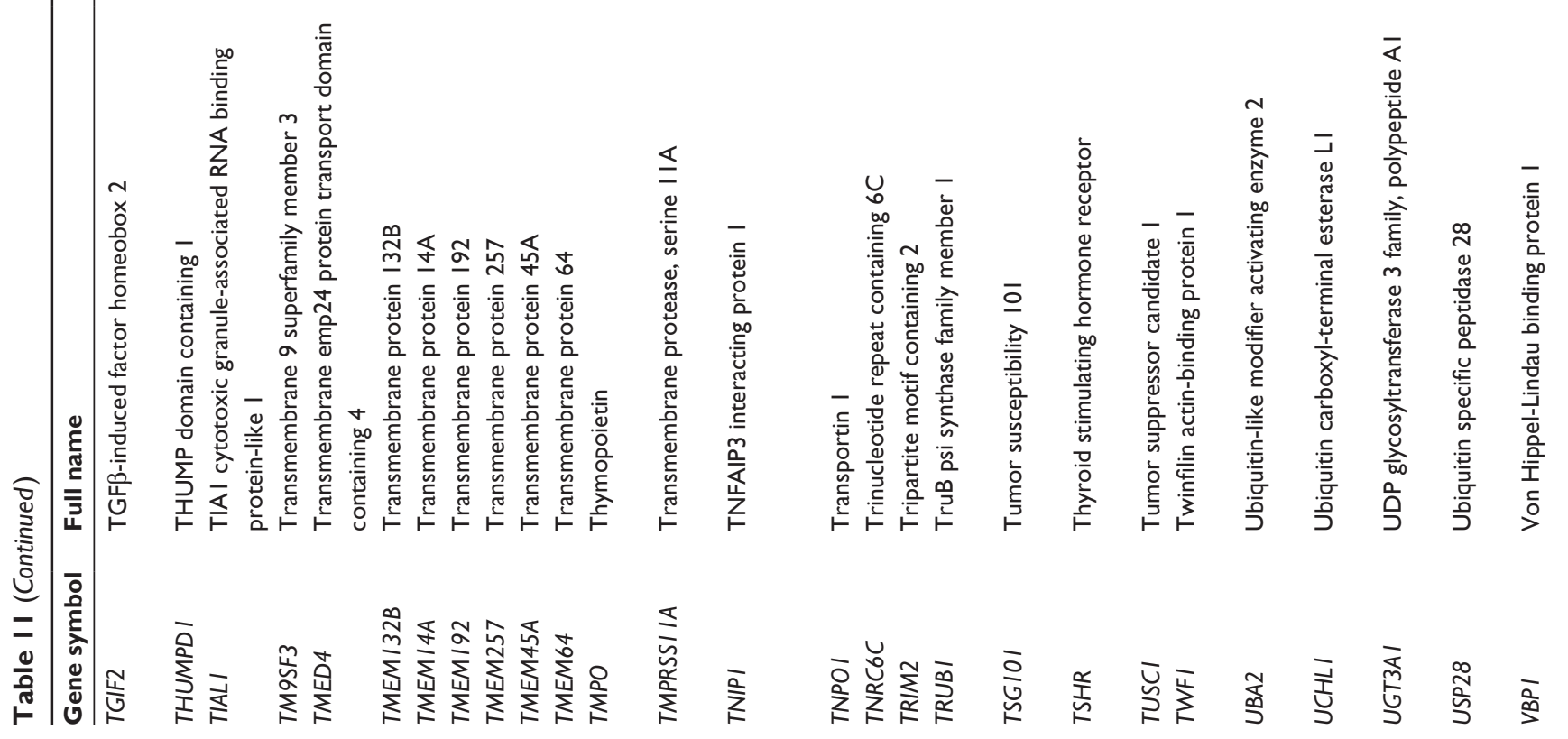



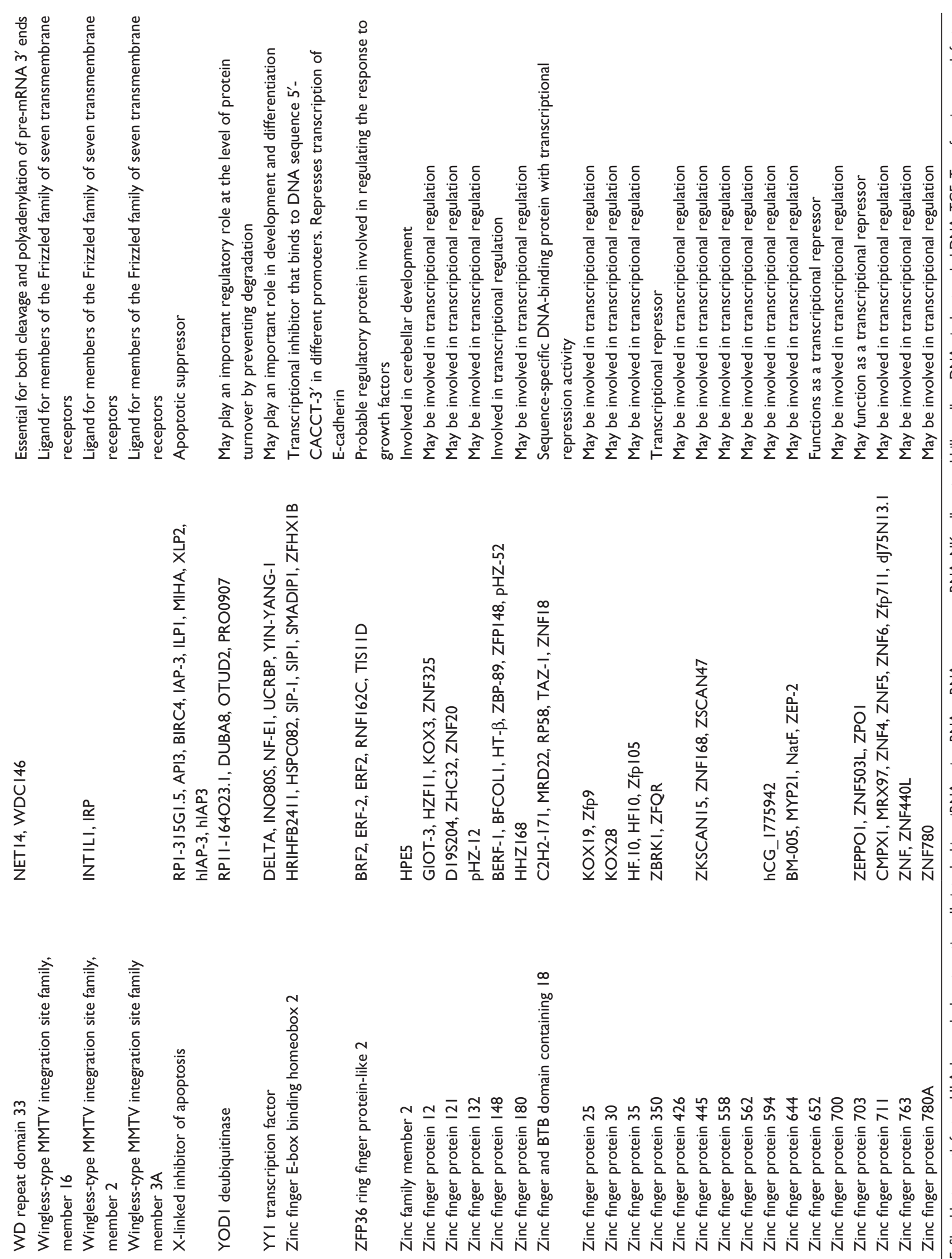

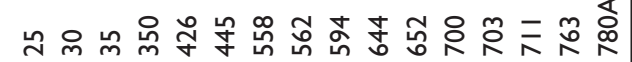

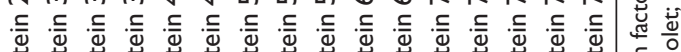

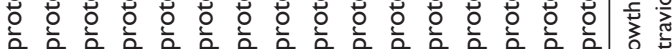

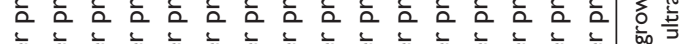

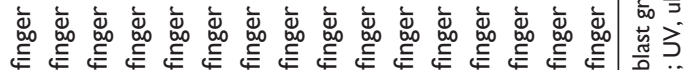

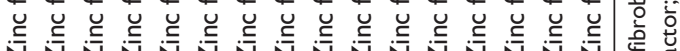
N $N$ N $N$ N $N$ N $N$ N $N$ N

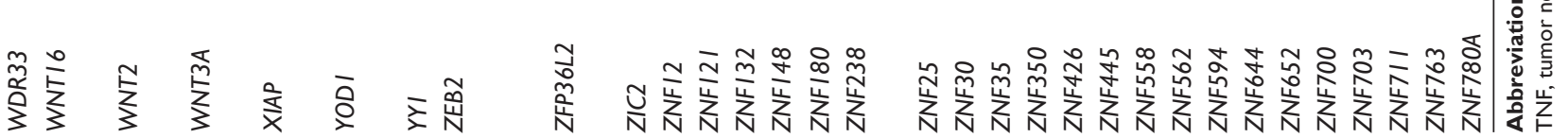


Table I 2 The top enriched clusters (enrich score $>$ I) by DAVID for the combined targets of hsa-miR-I8Ia-5p from both TarBase 6.0 and miRTarBase 4.0

\begin{tabular}{|c|c|c|c|c|}
\hline Category & Term & Gene count & $P$-value & FDR \\
\hline Annotation cluster I & Enrichment score: 4.49 & & & \\
\hline GOTERM_BP_FAT & Response to hormone stimulus & 7 & 7.70E-06 & I. I0E-03 \\
\hline GOTERM_BP_FAT & Response to endogenous stimulus & 7 & I.40E-05 & $1.50 \mathrm{E}-03$ \\
\hline GOTERM_BP_FAT & Response to organic substance & 7 & $3.30 \mathrm{E}-04$ & $8.80 \mathrm{E}-03$ \\
\hline Annotation cluster 2 & Enrichment score: 3.97 & & & \\
\hline GOTERM_BP_FAT & Negative regulation of apoptosis & 6 & I.00E-04 & $5.60 \mathrm{E}-03$ \\
\hline GOTERM_BP_FAT & Negative regulation of programmed cell death & 6 & I.IOE-04 & $5.00 \mathrm{E}-03$ \\
\hline GOTERM_BP_FAT & Negative regulation of cell death & 6 & I.IOE-04 & $4.80 \mathrm{E}-03$ \\
\hline Annotation cluster 3 & Enrichment score: 3.57 & & & \\
\hline GOTERM_BP_FAT & DNA damage checkpoint & 4 & $3.90 \mathrm{E}-05$ & 3. $10 \mathrm{E}-03$ \\
\hline GOTERM_BP_FAT & DNA integrity checkpoint & 4 & 5.00E-05 & $3.60 \mathrm{E}-03$ \\
\hline GOTERM_BP_FAT & DNA damage response, signal transduction & 4 & I.80E-04 & $6.50 \mathrm{E}-03$ \\
\hline GOTERM_BP_FAT & Cell cycle checkpoint & 4 & $2.60 \mathrm{E}-04$ & 7.20E-03 \\
\hline GOTERM_BP_FAT & Response to DNA damage stimulus & 4 & I.50E-02 & I.00E-0I \\
\hline Annotation cluster 4 & Enrichment score: 3.38 & & & \\
\hline KEGG_PATHWAY & Bladder cancer & 4 & I.80E-04 & $1.50 \mathrm{E}-03$ \\
\hline KEGG_PATHWAY & Endometrial cancer & 4 & 3.40E-04 & $2.30 \mathrm{E}-03$ \\
\hline KEGG_PATHWAY & Non-small-cell lung cancer & 4 & $3.80 \mathrm{E}-04$ & 2.30E-03 \\
\hline KEGG_PATHWAY & Acute myeloid leukemia & 4 & 4.70E-04 & 2.60E-03 \\
\hline KEGG_PATHWAY & Glioma & 4 & $6.00 \mathrm{E}-04$ & $3.00 \mathrm{E}-03$ \\
\hline KEGG_PATHWAY & Melanoma & 4 & $8.50 \mathrm{E}-04$ & $3.60 \mathrm{E}-03$ \\
\hline Annotation cluster $\mathbf{5}$ & Enrichment score: 3.2 & & & \\
\hline GOTERM_BP_FAT & Regulation of apoptosis & 7 & $6.00 \mathrm{E}-04$ & I.40E-02 \\
\hline GOTERM_BP_FAT & Regulation of programmed cell death & 7 & 6.30E-04 & I. $40 \mathrm{E}-02$ \\
\hline GOTERM_BP_FAT & Regulation of cell death & 7 & $6.50 \mathrm{E}-04$ & I. $40 \mathrm{E}-02$ \\
\hline Annotation cluster 6 & Enrichment score: 3.13 & & & \\
\hline GOTERM_BP_FAT & Developmental growth & 4 & $2.30 \mathrm{E}-04$ & $7.20 \mathrm{E}-03$ \\
\hline GOTERM_BP_FAT & Cell fate commitment & 4 & $9.10 \mathrm{E}-04$ & $1.90 \mathrm{E}-02$ \\
\hline GOTERM_BP_FAT & Tissue morphogenesis & 4 & $1.90 \mathrm{E}-03$ & $3.30 \mathrm{E}-02$ \\
\hline Annotation cluster 7 & Enrichment score: 2.74 & & & \\
\hline GOTERM_BP_FAT & Apoptosis & 6 & I.20E-03 & $2.20 \mathrm{E}-02$ \\
\hline GOTERM_BP_FAT & Programmed cell death & 6 & I.30E-03 & $2.30 \mathrm{E}-02$ \\
\hline GOTERM_BP_FAT & Cell death & 6 & $2.60 \mathrm{E}-03$ & $3.90 \mathrm{E}-02$ \\
\hline GOTERM_BP_FAT & Death & 6 & $2.70 \mathrm{E}-03$ & $3.80 \mathrm{E}-02$ \\
\hline Annotation cluster 8 & Enrichment score: 2.7 & & & \\
\hline GOTERM_BP_FAT & Positive regulation of macromolecule biosynthetic process & 6 & I.70E-03 & $3.00 \mathrm{E}-02$ \\
\hline GOTERM_BP_FAT & Positive regulation of cellular biosynthetic process & 6 & 2. I0E-03 & $3.50 \mathrm{E}-02$ \\
\hline GOTERM_BP_FAT & Positive regulation of biosynthetic process & 6 & $2.20 \mathrm{E}-03$ & $3.70 \mathrm{E}-02$ \\
\hline Annotation cluster 9 & Enrichment score: 2.56 & & & \\
\hline GOTERM_BP_FAT & Positive regulation of apoptosis & 5 & $2.70 \mathrm{E}-03$ & $3.90 \mathrm{E}-02$ \\
\hline GOTERM_BP_FAT & Positive regulation of programmed cell death & 5 & 2.70E-03 & $3.80 \mathrm{E}-02$ \\
\hline GOTERM_BP_FAT & Positive regulation of cell death & 5 & $2.80 \mathrm{E}-03$ & $3.80 \mathrm{E}-02$ \\
\hline Annotation cluster 10 & Enrichment score: $2.4 \mathrm{I}$ & & & \\
\hline GOTERM_BP_FAT & Regulation of phosphorylation & 5 & $3.60 \mathrm{E}-03$ & 4.40E-02 \\
\hline GOTERM_BP_FAT & Regulation of phosphate metabolic process & 5 & 4. I0E-03 & $4.50 \mathrm{E}-02$ \\
\hline GOTERM_BP_FAT & Regulation of phosphorus metabolic process & 5 & 4. $10 \mathrm{E}-03$ & $4.50 \mathrm{E}-02$ \\
\hline Annotation cluster I I & Enrichment score: 2.22 & & & \\
\hline GOTERM_BP_FAT & Positive regulation of transcription, DNA-dependent & 5 & $3.90 \mathrm{E}-03$ & $4.40 \mathrm{E}-02$ \\
\hline GOTERM_BP_FAT & Positive regulation of transcription & 5 & $7.00 \mathrm{E}-03$ & $6.70 \mathrm{E}-02$ \\
\hline GOTERM_BP_FAT & Positive regulation of gene expression & 5 & 7.80E-03 & 7.20E-02 \\
\hline Annotation cluster I 2 & Enrichment score: 2.15 & & & \\
\hline GOTERM_BP_FAT & Positive regulation of protein kinase activity & 4 & $3.50 \mathrm{E}-03$ & $4.40 \mathrm{E}-02$ \\
\hline GOTERM_BP_FAT & Positive regulation of kinase activity & 4 & $3.90 \mathrm{E}-03$ & $4.40 \mathrm{E}-02$ \\
\hline GOTERM_BP_FAT & Positive regulation of transferase activity & 4 & 4.30E-03 & $4.60 \mathrm{E}-02$ \\
\hline GOTERM_BP_FAT & Regulation of protein kinase activity & 4 & I.20E-02 & $9.20 \mathrm{E}-02$ \\
\hline GOTERM_BP_FAT & Regulation of kinase activity & 4 & I.30E-02 & $9.80 \mathrm{E}-02$ \\
\hline GOTERM_BP_FAT & Regulation of transferase activity & 4 & I.40E-02 & I.00E-0I \\
\hline
\end{tabular}


Table I 2 (Continued)

\begin{tabular}{|c|c|c|c|c|}
\hline Category & Term & Gene count & $P$-value & FDR \\
\hline Annotation cluster 13 & Enrichment score: $\mathbf{2 . 1}$ & & & \\
\hline GOTERM_BP_FAT & Positive regulation of cellular protein metabolic process & 4 & $4.00 \mathrm{E}-03$ & 4.40E-02 \\
\hline GOTERM_BP_FAT & Positive regulation of protein metabolic process & 4 & $4.50 \mathrm{E}-03$ & $4.70 \mathrm{E}-02$ \\
\hline GOTERM_BP_FAT & Regulation of cellular protein metabolic process & 4 & $2.70 \mathrm{E}-02$ & $\mathrm{I} .80 \mathrm{E}-0 \mathrm{I}$ \\
\hline Annotation cluster 14 & Enrichment score: 2.1 & & & \\
\hline GOTERM_BP_FAT & Branching morphogenesis of a tube & 3 & $3.70 \mathrm{E}-03$ & $4.30 \mathrm{E}-02$ \\
\hline GOTERM_BP_FAT & Positive regulation of cell development & 3 & 4. I0E-03 & $4.50 \mathrm{E}-02$ \\
\hline GOTERM_BP_FAT & Morphogenesis of a branching structure & 3 & $4.80 \mathrm{E}-03$ & $4.90 \mathrm{E}-02$ \\
\hline GOTERM_BP_FAT & Tube morphogenesis & 3 & I.30E-02 & $\mathrm{I} .00 \mathrm{E}-0 \mathrm{I}$ \\
\hline GOTERM_BP_FAT & Regulation of cell development & 3 & $3.30 \mathrm{E}-02$ & $2.00 \mathrm{E}-0 \mathrm{I}$ \\
\hline Annotation cluster I5 & Enrichment score: 2.06 & & & \\
\hline GOTERM_BP_FAT & Neuron projection morphogenesis & 4 & 3. $10 \mathrm{E}-03$ & $4.00 \mathrm{E}-02$ \\
\hline GOTERM_BP_FAT & Cell projection morphogenesis & 4 & $4.60 \mathrm{E}-03$ & $4.80 \mathrm{E}-02$ \\
\hline GOTERM_BP_FAT & Neuron projection development & 4 & $5.20 \mathrm{E}-03$ & $5.10 \mathrm{E}-02$ \\
\hline GOTERM_BP_FAT & Cell part morphogenesis & 4 & $5.20 \mathrm{E}-03$ & 5. $10 \mathrm{E}-02$ \\
\hline GOTERM_BP_FAT & Neuron development & 4 & I. I0E-02 & $9.00 \mathrm{E}-02$ \\
\hline GOTERM_BP_FAT & Cell morphogenesis & 4 & I.30E-02 & $9.80 \mathrm{E}-02$ \\
\hline GOTERM_BP_FAT & Cell projection organization & 4 & I.40E-02 & I.00E-0 I \\
\hline GOTERM_BP_FAT & Cellular component morphogenesis & 4 & I.70E-02 & I.20E-0I \\
\hline GOTERM_BP_FAT & Neuron differentiation & 4 & $2.20 \mathrm{E}-02$ & $\mathrm{I} .50 \mathrm{E}-0 \mathrm{I}$ \\
\hline Annotation cluster 16 & Enrichment score: 2.03 & & & \\
\hline BIOCARTA & IGF-I signaling pathway & 3 & $7.00 \mathrm{E}-03$ & $3.00 \mathrm{E}-0 \mathrm{I}$ \\
\hline BIOCARTA & IL-6 signaling pathway & 3 & $7.00 \mathrm{E}-03$ & $3.00 \mathrm{E}-0 \mathrm{I}$ \\
\hline BIOCARTA & Insulin signaling pathway & 3 & $7.00 \mathrm{E}-03$ & $3.00 \mathrm{E}-0 \mathrm{I}$ \\
\hline BIOCARTA & Signaling of hepatocyte growth factor receptor & 3 & $2.20 \mathrm{E}-02$ & 3. IOE-0I \\
\hline Annotation cluster 17 & Enrichment score: 1.97 & & & \\
\hline GOTERM_BP_FAT & Embryonic appendage morphogenesis & 3 & $6.50 \mathrm{E}-03$ & $6.30 \mathrm{E}-02$ \\
\hline GOTERM_BP_FAT & Embryonic limb morphogenesis & 3 & $6.50 \mathrm{E}-03$ & $6.30 \mathrm{E}-02$ \\
\hline GOTERM_BP_FAT & Limb morphogenesis & 3 & $8.40 \mathrm{E}-03$ & $7.50 \mathrm{E}-02$ \\
\hline GOTERM_BP_FAT & Appendage morphogenesis & 3 & $8.40 \mathrm{E}-03$ & $7.50 \mathrm{E}-02$ \\
\hline GOTERM_BP_FAT & Limb development & 3 & $9.00 \mathrm{E}-03$ & $7.70 \mathrm{E}-02$ \\
\hline GOTERM_BP_FAT & Appendage development & 3 & $9.00 \mathrm{E}-03$ & $7.70 \mathrm{E}-02$ \\
\hline GOTERM_BP_FAT & Embryonic morphogenesis & 3 & $6.80 \mathrm{E}-02$ & $3.40 \mathrm{E}-0 \mathrm{I}$ \\
\hline Annotation cluster 18 & Enrichment score: I.8I & & & \\
\hline GOTERM_BP_FAT & Response to ethanol & 3 & $3.60 \mathrm{E}-03$ & $4.30 \mathrm{E}-02$ \\
\hline GOTERM_BP_FAT & Response to metal ion & 3 & I.40E-02 & $\mathrm{I} .00 \mathrm{E}-0 \mathrm{I}$ \\
\hline GOTERM_BP_FAT & Response to inorganic substance & 3 & $3.30 \mathrm{E}-02$ & $2.00 \mathrm{E}-0 \mathrm{I}$ \\
\hline GOTERM_BP_FAT & Response to drug & 3 & $3.60 \mathrm{E}-02$ & 2. IOE-0I \\
\hline Annotation cluster 19 & Enrichment score: 1.59 & & & \\
\hline GOTERM_BP_FAT & Response to estrogen stimulus & 3 & $9.40 \mathrm{E}-03$ & $7.90 \mathrm{E}-02$ \\
\hline GOTERM_BP_FAT & Positive regulation of protein modification process & 3 & $2.80 \mathrm{E}-02$ & I.80E-0। \\
\hline GOTERM_BP_FAT & Regulation of protein modification process & 3 & $6.40 \mathrm{E}-02$ & $3.20 \mathrm{E}-0 \mathrm{I}$ \\
\hline Annotation cluster 20 & Enrichment score: 1.57 & & & \\
\hline GOTERM_BP_FAT & Protein amino acid phosphorylation & 5 & I.30E-02 & $9.70 \mathrm{E}-02$ \\
\hline GOTERM_BP_FAT & Phosphorylation & 5 & $2.30 \mathrm{E}-02$ & I.50E-0 I \\
\hline GOTERM_BP_FAT & Phosphate metabolic process & 5 & $4.30 \mathrm{E}-02$ & $2.40 \mathrm{E}-0 \mathrm{I}$ \\
\hline GOTERM_BP_FAT & Phosphorus metabolic process & 5 & $4.30 \mathrm{E}-02$ & $2.40 \mathrm{E}-0 \mathrm{I}$ \\
\hline Annotation cluster 21 & Enrichment score: I.55 & & & \\
\hline GOTERM_BP_FAT & Cell aging & 3 & $9.60 \mathrm{E}-04$ & $1.90 \mathrm{E}-02$ \\
\hline GOTERM_BP_FAT & Negative regulation of neuron apoptosis & 3 & $2.30 \mathrm{E}-03$ & $3.70 \mathrm{E}-02$ \\
\hline GOTERM_BP_FAT & Aging & 3 & I.00E-02 & $8.50 \mathrm{E}-02$ \\
\hline GOTERM_BP_FAT & Actin cytoskeleton organization & 3 & $3.90 \mathrm{E}-02$ & $2.20 \mathrm{E}-0 \mathrm{I}$ \\
\hline GOTERM_BP_FAT & Actin filament-based process & 3 & $4.40 \mathrm{E}-02$ & $2.40 \mathrm{E}-0 \mathrm{I}$ \\
\hline GOTERM_BP_FAT & Membrane organization & 3 & $9.90 \mathrm{E}-02$ & $4.40 \mathrm{E}-0 \mathrm{I}$ \\
\hline GOTERM_CC_FAT & Membrane fraction & 3 & $3.20 \mathrm{E}-0 \mathrm{I}$ & $9.40 \mathrm{E}-0 \mathrm{I}$ \\
\hline GOTERM_CC_FAT & Insoluble fraction & 3 & $3.30 \mathrm{E}-0 \mathrm{I}$ & $9.30 \mathrm{E}-0 \mathrm{I}$ \\
\hline Annotation cluster 22 & Enrichment score: 1.47 & & & \\
\hline GOTERM_BP_FAT & Ras protein signal transduction & 3 & $9.40 \mathrm{E}-03$ & $7.90 \mathrm{E}-02$ \\
\hline KEGG_PATHWAY & Long-term depression & 3 & I.50E-02 & 4.40E-02 \\
\hline
\end{tabular}

(Continued) 
Table 12 (Continued)

\begin{tabular}{|c|c|c|c|c|}
\hline Category & Term & Gene count & $P$-value & FDR \\
\hline KEGG_PATHWAY & B cell receptor signaling pathway & 3 & I.70E-02 & $4.50 \mathrm{E}-02$ \\
\hline KEGG_PATHWAY & VEGF signaling pathway & 3 & I.70E-02 & $4.50 \mathrm{E}-02$ \\
\hline KEGG_PATHWAY & Fc epsilon RI signaling pathway & 3 & $1.90 \mathrm{E}-02$ & $4.60 \mathrm{E}-02$ \\
\hline KEGG_PATHWAY & ErbB signaling pathway & 3 & $2.30 \mathrm{E}-02$ & $5.40 \mathrm{E}-02$ \\
\hline KEGG_PATHWAY & Gap junction & 3 & $2.40 \mathrm{E}-02$ & $5.50 \mathrm{E}-02$ \\
\hline KEGG_PATHWAY & GnRH signaling pathway & 3 & $2.90 \mathrm{E}-02$ & $6.30 \mathrm{E}-02$ \\
\hline KEGG_PATHWAY & T-cell receptor signaling pathway & 3 & $3.40 \mathrm{E}-02$ & 7.20E-02 \\
\hline KEGG_PATHWAY & Insulin signaling pathway & 3 & $5.20 \mathrm{E}-02$ & I.00E-0I \\
\hline GOTERM_BP_FAT & Small GTPase-mediated signal transduction & 3 & $6.70 \mathrm{E}-02$ & $3.40 \mathrm{E}-0 \mathrm{I}$ \\
\hline KEGG_PATHWAY & Chemokine signaling pathway & 3 & $9.20 \mathrm{E}-02$ & I.70E-0I \\
\hline KEGG_PATHWAY & Regulation of actin cytoskeleton & 3 & I.20E-0I & 2. IOE-0I \\
\hline KEGG_PATHWAY & MAPK signaling pathway & 3 & I.70E-0I & $2.80 \mathrm{E}-0 \mathrm{I}$ \\
\hline Annotation cluster 23 & Enrichment score: I.45 & & & \\
\hline GOTERM_BP_FAT & Axonogenesis & 3 & $3.00 \mathrm{E}-02$ & I.80E-0। \\
\hline GOTERM_BP_FAT & Cell morphogenesis involved in neuron differentiation & 3 & $3.40 \mathrm{E}-02$ & 2.00E-0I \\
\hline GOTERM_BP_FAT & Cell morphogenesis involved in differentiation & 3 & 4.50E-02 & $2.50 \mathrm{E}-0 \mathrm{I}$ \\
\hline Annotation cluster 24 & Enrichment score: I.3I & & & \\
\hline GOTERM_CC_FAT & Nucleoplasm & 6 & $6.20 \mathrm{E}-03$ & $4.90 \mathrm{E}-0 \mathrm{I}$ \\
\hline GOTERM_CC_FAT & Nuclear lumen & 6 & $4.50 \mathrm{E}-02$ & $5.70 \mathrm{E}-0 \mathrm{I}$ \\
\hline GOTERM_CC_FAT & Intracellular organelle lumen & 6 & $9.40 \mathrm{E}-02$ & 7.40E-0I \\
\hline GOTERM_CC_FAT & Organelle lumen & 6 & I.00E-0I & $7.20 \mathrm{E}-0 \mathrm{I}$ \\
\hline GOTERM_CC_FAT & Membrane-enclosed lumen & 6 & I.IOE-0I & 7.IOE-0I \\
\hline Annotation cluster 25 & Enrichment score: 1.3 & & & \\
\hline GOTERM_BP_FAT & Hemopoiesis & 3 & 4.30E-02 & $2.40 \mathrm{E}-0 \mathrm{I}$ \\
\hline GOTERM_BP_FAT & Hemopoietic or lymphoid organ development & 3 & 5. I0E-02 & 2.70E-0I \\
\hline GOTERM_BP_FAT & Immune system development & 3 & $5.60 \mathrm{E}-02$ & $2.90 \mathrm{E}-0 \mathrm{I}$ \\
\hline Annotation cluster 26 & Enrichment score: I.06 & & & \\
\hline SP_PIR_KEYWORDS & Transcription regulation & 6 & $6.30 \mathrm{E}-02$ & $3.80 \mathrm{E}-0 \mathrm{I}$ \\
\hline SP_PIR_KEYWORDS & Transcription & 6 & $6.80 \mathrm{E}-02$ & $3.90 \mathrm{E}-0 \mathrm{I}$ \\
\hline GOTERM_BP_FAT & Transcription & 6 & I.60E-0| & 6.IOE-0I \\
\hline
\end{tabular}

Abbreviations: DAVID, Database for Annotation, Visualization and Integrated Discovery; FDR, false discovery rate; GnRH, gonadotropin releasing hormone; IGF, insulinlike growth factor; IL, interleukin; VEGF, vascular endothelial growth factor.

nuclear lumen, intracellular organelle lumen, organelle lumen, membrane-enclosed lumen, hemopoiesis, hemopoietic or lymphoid organ development, immune system development, and transcription regulation (Table 12).

Furthermore, our DAVID analysis revealed that there were 33 KEGG pathways significantly enriched in the target list of hsa-miR-181a-5p, based on both TarBase and miRTarBase (Table 13). These pathways included pathways in cancer, prostate cancer, thyroid cancer, renal cell carcinoma, chronic myeloid leukemia, neurotrophin signaling pathway, dorsoventral axis formation, bladder cancer, endometrial cancer, non-small-cell lung cancer, acute myeloid leukemia, glioma, long-term potentiation, melanoma, colorectal cancer, melanogenesis, cell cycle, natural killer cell-mediated cytotoxicity, focal adhesion, notch signaling pathway, longterm depression, pancreatic cancer, B cell receptor signaling pathway, VEGF signaling pathway, Fc epsilon RI signaling pathway, ErbB signaling pathway, gap junction, GnRH signaling pathway, $\mathrm{T}$ cell receptor signaling pathway, insulin signaling pathway, Jak-STAT signaling pathway, chemokine signaling pathway, and prion diseases.

Among the 313 validated targets of hsa-miR-181a-5p, 22 were cancer genes (Table 14). These included ATM, BCL2, BRCA1, CCDC6, CCND1, CDX2, EP300, FBXO11, H3F3B, HOOK3, HOXA11, HRAS, KRAS, MAP2K1, NOTCH1, NOTCH2, PLAG1, PTPN11, RBM15, STAG2, TAF15, and TSHR. The KEGG pathway analysis also indicate that the targets regulated by hsa-miR-181a that are cancer genes are all involved in the tumorigenesis of bladder cancer, endometrial cancer, non-small-cell lung cancer, acute myeloid leukemia, glioma, melanoma, and colorectal cancer.

Now when we looked at the prediction accuracy of all the nine algorithms we used, we found that all the predicting programs behaved poorly. The reasons for this may include: a) the predicting criteria were not set up properly; b) the matching criteria for hsa-miR-181a with the complementary sites of the target mRNAs may have been too restrictive or too loose; c) the value for the threshold was not properly set prior to prediction; 

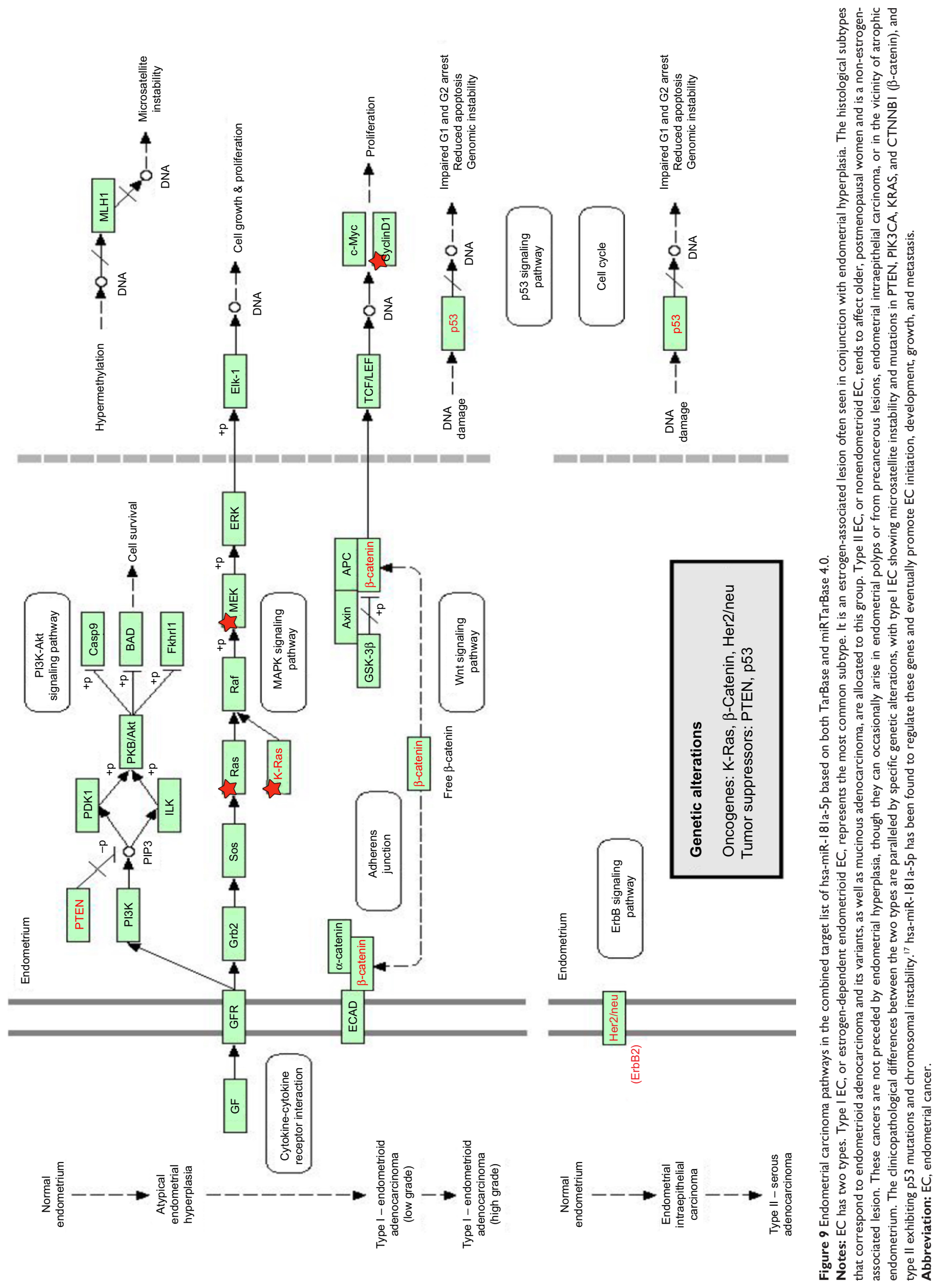
Table 13 KEGG pathways for the combined targets of hsa-miR-18Ia-5p based on TarBase 6.0 and miRTarBase 4.0

\begin{tabular}{|c|c|c|c|c|}
\hline Signaling pathway & Gene count & $\%$ & $P$-value & FDR \\
\hline Pathways in cancer & 7 & 31.8 & I.30E-04 & I.30E-03 \\
\hline Prostate cancer & 6 & 27.3 & $2.60 \mathrm{E}-06$ & $7.80 \mathrm{E}-05$ \\
\hline Thyroid cancer & 5 & 22.7 & $8.20 \mathrm{E}-07$ & $4.90 \mathrm{E}-05$ \\
\hline Renal cell carcinoma & 5 & 22.7 & $3.00 \mathrm{E}-05$ & $5.90 \mathrm{E}-04$ \\
\hline Chronic myeloid leukemia & 5 & 22.7 & $3.90 \mathrm{E}-05$ & 4.70E-04 \\
\hline Neurotrophin signaling pathway & 5 & 22.7 & $2.80 \mathrm{E}-04$ & 2. $10 \mathrm{E}-03$ \\
\hline Dorsoventral axis formation & 4 & 18.2 & 3.70E-05 & $5.50 \mathrm{E}-04$ \\
\hline Bladder cancer & 4 & 18.2 & I.80E-04 & I.50E-03 \\
\hline Endometrial cancer & 4 & 18.2 & $3.40 \mathrm{E}-04$ & $2.30 \mathrm{E}-03$ \\
\hline Non-small-cell lung cancer & 4 & 18.2 & $3.80 \mathrm{E}-04$ & $2.30 \mathrm{E}-03$ \\
\hline Acute myeloid leukemia & 4 & 18.2 & 4.70E-04 & $2.60 \mathrm{E}-03$ \\
\hline Glioma & 4 & 18.2 & $6.00 \mathrm{E}-04$ & $3.00 \mathrm{E}-03$ \\
\hline Long-term potentiation & 4 & 18.2 & $7.50 \mathrm{E}-04$ & $3.50 \mathrm{E}-03$ \\
\hline Melanoma & 4 & 18.2 & 8.50E-04 & $3.60 \mathrm{E}-03$ \\
\hline Colorectal cancer & 4 & 18.2 & I.40E-03 & $5.50 \mathrm{E}-03$ \\
\hline Melanogenesis & 4 & 18.2 & $2.20 \mathrm{E}-03$ & $8.30 \mathrm{E}-03$ \\
\hline Cell cycle & 4 & 18.2 & 4.30E-03 & I.50E-02 \\
\hline Natural killer cell-mediated cytotoxicity & 4 & 18.2 & $5.20 \mathrm{E}-03$ & I.70E-02 \\
\hline Focal adhesion & 4 & 18.2 & $1.60 \mathrm{E}-02$ & $4.50 \mathrm{E}-02$ \\
\hline Notch signaling pathway & 3 & 13.6 & $7.10 \mathrm{E}-03$ & $2.20 \mathrm{E}-02$ \\
\hline Long-term depression & 3 & 13.6 & I.50E-02 & $4.40 \mathrm{E}-02$ \\
\hline Pancreatic cancer & 3 & 13.6 & I.60E-02 & $4.30 \mathrm{E}-02$ \\
\hline B-cell receptor signaling pathway & 3 & 13.6 & I.70E-02 & $4.50 \mathrm{E}-02$ \\
\hline VEGF signaling pathway & 3 & 13.6 & I.70E-02 & $4.50 \mathrm{E}-02$ \\
\hline Fc epsilon RI signaling pathway & 3 & 13.6 & $1.90 \mathrm{E}-02$ & $4.60 \mathrm{E}-02$ \\
\hline ErbB signaling pathway & 3 & 13.6 & $2.30 \mathrm{E}-02$ & $5.40 \mathrm{E}-02$ \\
\hline Gap junction & 3 & 13.6 & $2.40 \mathrm{E}-02$ & $5.50 \mathrm{E}-02$ \\
\hline GnRH signaling pathway & 3 & 13.6 & $2.90 \mathrm{E}-02$ & $6.30 \mathrm{E}-02$ \\
\hline T cell receptor signaling pathway & 3 & 13.6 & $3.40 \mathrm{E}-02$ & $7.20 \mathrm{E}-02$ \\
\hline Insulin signaling pathway & 3 & 13.6 & $5.20 \mathrm{E}-02$ & I.00E-0I \\
\hline Jak-STAT signaling pathway & 3 & 13.6 & $6.60 \mathrm{E}-02$ & I.30E-0I \\
\hline Chemokine signaling pathway & 3 & 13.6 & $9.20 \mathrm{E}-02$ & I.70E-0I \\
\hline Prion diseases & 2 & 9.1 & $9.20 \mathrm{E}-02$ & I.70E-0I \\
\hline
\end{tabular}

Abbreviations: FDR, false discovery rate; GnRH, gonadotropin releasing hormone; KEGG, Kyoto Encyclopedia of Genes and Genomes; VEGF, vascular endothelial growth factor.

and d) the calculation of the $P$-values may have been too simple or too complicated. The poor predictive ability for all these algorithms also emphasizes the importance of experimental validation of the targets of any specific miRNAs.

\section{Clinical validation of the role of hsa-miR- I8Ia in EC tumorigenesis}

Next, we aimed to validate the function of hsa-miR-181a in the pathogenesis of EC by measuring and comparing the expression levels of hsa-miR-181a in normal, benign, and malignant endometrial tissues. The association of disease progression of EC with the expression profile of hsa-miR-181a was also determined. There are two types of EC with distinct histological characteristics. ${ }^{15,51}$ Herein, a total of 78 tissue samples were classified by immunohistochemical staining. There were 47 , 18 , and 13 samples that were categorized as EC, endometrial hyperplasia, or normal endometrium, respectively. For the EC group, there were 37 samples, and 10 samples that belonged to type I and type II EC, respectively. The type I EC was ERand PR-positive (Figure 10), and the type II EC was ER- and PR-negative (Figure 11).

To examine the potential role of hsa-miR-181a in the development and progression of EC, the expression profile of hsa-miR-181a was tested in all collected clinical samples, using RT-PCR (Figure S1). In comparison with normal endometrium, the expression level of hsa-miR-181a was increased 8.5-, 31.2-, and 4.1-fold in type I EC, type II EC, and endometrial hyperplasia, respectively $(P<0.05$, by oneway ANOVA) (Tables 15 and 16). The expression level of hsa-miR-181a in type II EC was higher (3.7-fold) than that in type I EC $(P<0.05)$. In addition, hsa-miR-181a had a higher expression level in EC than that in endometrial hyperplasia.

Since we have observed the differential expression profile of hsa-miR-181a in all examined clinical samples, we further 
Table 14 Combined targets of hsa-miR-18Ia-5p based on TarBase 6.0 and miRTarBase 4.0, that are cancer genes

\begin{tabular}{|c|c|c|c|}
\hline Gene symbol & Name & Tumor types (somatic) & Tumor types (germline) \\
\hline ATM & Ataxia telangiectasia mutated & T-PLL & $\begin{array}{l}\text { Leukemia; lymphoma; } \\
\text { medulloblastoma; glioma }\end{array}$ \\
\hline$B C L 2$ & B-cell CLL/lymphoma 2 & NHL; CLL & \\
\hline BRCAI & Familial breast/ovarian cancer gene I & Ovarian & Breast; ovarian \\
\hline CCDC6 & Coiled-coil domain containing 6 & NSCLC & \\
\hline CCNDI & Cyclin DI & CLL; B-ALL; breast & \\
\hline$C D \times 2$ & Caudal type homeobox transcription factor 2 & AML & \\
\hline EP300 & $300 \mathrm{kDa}$ EIA-binding protein gene & $\begin{array}{l}\text { Colorectal; breast; pancreatic; AML; ALL; } \\
\text { DLBCL }\end{array}$ & \\
\hline FBXOII & F-box protein II & DLBCL & \\
\hline H3F3B & $\mathrm{H} 3$ histone; family 3B (H3.3B) & Chondroblastoma & \\
\hline НООКЗ & Hook homolog 3 & Papillary thyroid & \\
\hline HOXAII & Homeobox AII & CML & \\
\hline HRAS & $\begin{array}{l}\text { v-Ha-ras Harvey rat sarcoma viral oncogene } \\
\text { homolog }\end{array}$ & $\begin{array}{l}\text { Infrequent sarcomas; rare other tumor } \\
\text { types }\end{array}$ & $\begin{array}{l}\text { Rhabdomyosarcoma; } \\
\text { ganglioneuroblastoma; bladder }\end{array}$ \\
\hline KRAS & $\begin{array}{l}\text { v-Ki-ras2 Kirsten rat sarcoma } 2 \text { viral } \\
\text { oncogene homolog }\end{array}$ & $\begin{array}{l}\text { Pancreatic; colorectal; lung; thyroid; AML; } \\
\text { other tumor types }\end{array}$ & \\
\hline MAP2KI & Mitogen-activated protein kinase kinase I & NSCLC; melanoma; colorectal & \\
\hline $\mathrm{NOTCHI}$ & $\begin{array}{l}\text { Notch homolog I; translocation-associated } \\
\text { (Drosophila) (TANI) }\end{array}$ & T-ALL & \\
\hline $\mathrm{NOTCH} 2$ & Notch homolog 2 & Marginal zone lymphoma; DLBCL & \\
\hline PLAGI & Pleiomorphic adenoma gene I & Salivary adenoma & \\
\hline PTPNII & $\begin{array}{l}\text { Protein tyrosine phosphatase; nonreceptor } \\
\text { type II }\end{array}$ & JMML; AML; MDS & \\
\hline RBM $/ 5$ & RNA binding motif protein 15 & Acute megakaryocytic leukemia & \\
\hline STAG2 & Stromal antigen 2 & $\begin{array}{l}\text { Bladder carcinoma; glioblastoma; } \\
\text { melanoma; Ewing's sarcoma; myeloid } \\
\text { neoplasms }\end{array}$ & \\
\hline TAFI5 & $\begin{array}{l}\text { TAFI5 RNA polymerase II; TBP-associated } \\
\text { factor; } 68 \mathrm{kDa}\end{array}$ & $\begin{array}{l}\text { Extraskeletal myxoid chondrosarcoma; } \\
\text { ALL }\end{array}$ & \\
\hline TSHR & Thyroid stimulating hormone receptor & Toxic thyroid adenoma & Thyroid adenoma \\
\hline
\end{tabular}

Abbreviations: ALL, acute lymphocytic leukemia; AML, adult acute myeloid leukemia; B-ALL, B-cell ALL; CLL, chronic lymphocytic leukemia; CML, chronic myelogenous leukemia; DLBCL, diffuse large B-cell lymphoma; JMML, juvenile myelomonocytic leukemia; MDS, myelodysplastic syndrome; NHL, non-Hodgkin lymphoma; NSCLC, nonsmall-cell lung cancer; T-ALL, T-cell ALL; T-PLL, T-cell subtype of prolymphocytic leukemia.
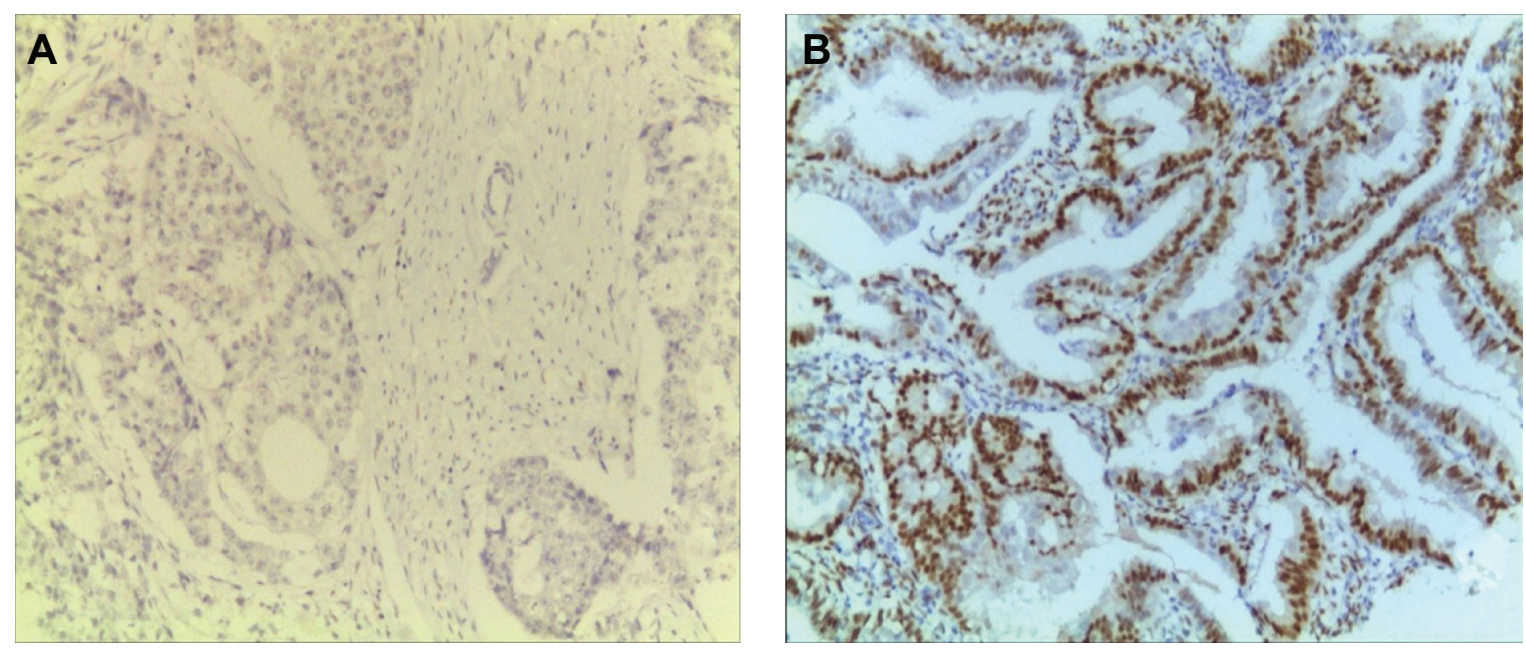

Figure 10 The expression of ER and PR in type I EC.

Notes: The expression of ER and PR was evaluated by immunohistochemistry. Dewaxed and dehydrated sections were washed with PBS and then, incubated with $3 \%$ peroxyl in methanol to terminate the activity of endogenous peroxidases. The sections were washed with PBS and immersed into boiled citrate-buffered solution for 10 minutes. The sections were blocked with $5 \%$ bovine serum albumin in PBS for 20 minutes at room temperature. Following that, the sections were probed with primary antibody against ER or PR, followed by biotinylated second anti-rabbit antibody. (A) Positive expression of ER; and (B) positive expression of PR.

Abbreviations: EC, endometrial cancer; ER, estrogen receptor; PBS, phosphate-buffered saline; PR, progesterone receptor. 

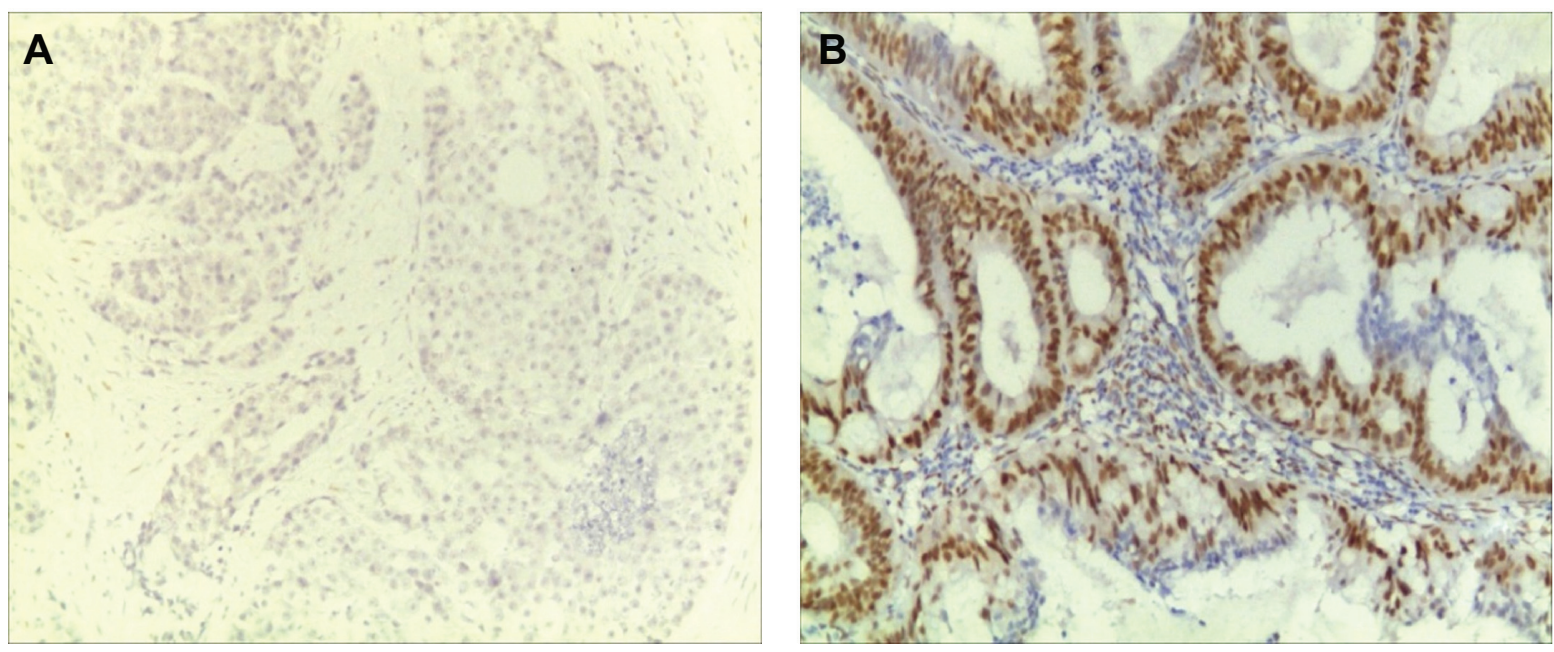

Figure II The expression of ER and PR in type II EC.

Notes: The expression of ER and PR was evaluated by immunohistochemistry. Dewaxed and dehydrated sections were washed with PBS and then, incubated with $3 \%$ peroxyl in methanol to terminate the activity of endogenous peroxidases. The sections were washed with PBS and immersed into boiled citrate-buffered solution for 10 minutes. The sections were blocked with $5 \%$ bovine serum albumin in PBS for 20 minutes at room temperature. Following that, the sections were probed with primary antibody against ER or PR, followed by biotinylated second anti-rabbit antibody. (A) Negative expression of ER; and (B) negative expression of PR.

Abbreviations: EC, endometrial cancer; ER, estrogen receptor; PBS, phosphate-buffered saline; PR, progesterone receptor.

assessed the association between the expression of hsa-miR181a and the development of EC with regard to histological type, stage, grade, migration, and invasion. As shown in Table 17, compared with the stage I and II EC, there was a remarkable increase in the expression level of hsa-miR-181a in stage III and VI EC ( $P=0.01$, by one-way ANOVA). The expression level of hsa-miR-181a was increased in EC with lymph migration and myometrial invasion. In addition, compared with the grade $1 \mathrm{EC}$, there was an increase in the expression level of hsa-miR-181a in grade 2 and grade $3 \mathrm{EC}(P>0.05$; Table 17). Taken together, these results indicate that there is an association between the expression level of hsa-miR-181a and the clinical development and progression of EC.

\section{Discussion}

miRNAs play critical roles in regulating proliferation, differentiation, apoptosis, development, metabolism, and immunity. ${ }^{2}$ miRNAs may act as oncogenes or tumor suppressors, and they could play a potential role as diagnostic and prognostic

Table I5 Expression level of hsa-miR-I8Ia in normal endometrium, endometrial hyperplasia, and EC

\begin{tabular}{llll}
\hline Tissue & $\mathbf{N}$ & $\Delta \mathbf{C t}$ & $\mathbf{2}^{-\Delta \Delta \mathrm{Ct}}$ \\
\hline Endometrial cancer & 47 & $-3.356 \pm 2.40 \mathrm{I}$ & 10.240 \\
Endometrial hyperplasia & 18 & $-1.893 \pm 2.568$ & 4.073 \\
Normal endometrium & 13 & $0.133 \pm 2.527$ & 1.000 \\
\hline
\end{tabular}

Note: $\Delta \mathrm{Ct}$, normalized threshold cycle; $2^{-\Delta \Delta \mathrm{C} t}$, a method used to calculate relative changes in the gene expression determined from real-time quantitative polymerase chain reaction experiments and it means the fold change.

Abbreviations: $\Delta \mathrm{Ct}$, normalized threshold cycle; $\mathrm{EC}$, endometrial cancer. biomarkers of cancers. ${ }^{7,52}$ Specific miRNAs are expressed in various tissues, and changes in regulation of gene expression are thought to cause carcinogenesis. Thus, tissue-specific miRNAs may be used as effective biomarkers for cancer diagnosis, treatment, and prognosis. ${ }^{8}$ Hsa-miR-181a has been proposed to play a role in the pathogenesis, development, progression, metastasis, prognosis, and therapeutic response to chemo- and radiotherapy in $\mathrm{EC},{ }^{4,53}$ ovarian cancer ${ }^{54}$ glioma, ${ }^{30,55}$ liver cancer, ${ }^{56}$ colorectal cancer,,${ }^{57-59}$ gastric cancer, ${ }^{60,61}$ lung cancer, ${ }^{62}$ breast cancer, ${ }^{63-67}$ cervical carcinoma, ${ }^{68,69}$ pancreatic cancer, ${ }^{46}$ osteosarcoma, ${ }^{70}$ oral squamous cell carcinoma, ${ }^{71,72}$ B-cell lymphoma, ${ }^{73}$ thyroid cancer, ${ }^{74}$ salivary adenoid cystic carcinoma,${ }^{75}$ and acute

Table 16 Differential expression level of hsa-miRI8la in endometrium tissues

\begin{tabular}{lll}
\hline Tissue & $\mathbf{2}^{-\Delta \Delta \mathrm{ct}}$ & $\mathbf{P}$ \\
\hline $\begin{array}{l}\text { Endometrial hyperplasia vs } \\
\text { normal endometrial tissue }\end{array}$ & 4.073 & 0.027 \\
$\begin{array}{l}\text { Type II endometrial cancer vs } \\
\text { type I endometrial cancer } \\
\begin{array}{l}\text { Type II endometrial cancer vs } \\
\text { endometrial hyperplasia }\end{array}\end{array}$ & 3.668 & 0.032 \\
$\begin{array}{l}\text { Type II endometrial cancer vs } \\
\text { normal endometrial tissue }\end{array}$ & 7.669 & 0.003 \\
$\begin{array}{l}\text { Type I endometrial cancer vs } \\
\text { endometrial hyperplasia }\end{array}$ & 31.233 & 0.000 \\
$\begin{array}{l}\text { Type I endometrial cancer vs } \\
\text { normal endometrial tissue }\end{array}$ & 2.091 & 0.127 \\
\hline
\end{tabular}

Note: $2^{-\Delta \Delta \mathrm{Ct}}$ a method used to calculate relative changes in the gene expression determined from real-time quantitative polymerase chain reaction experiments and it means the fold change. 


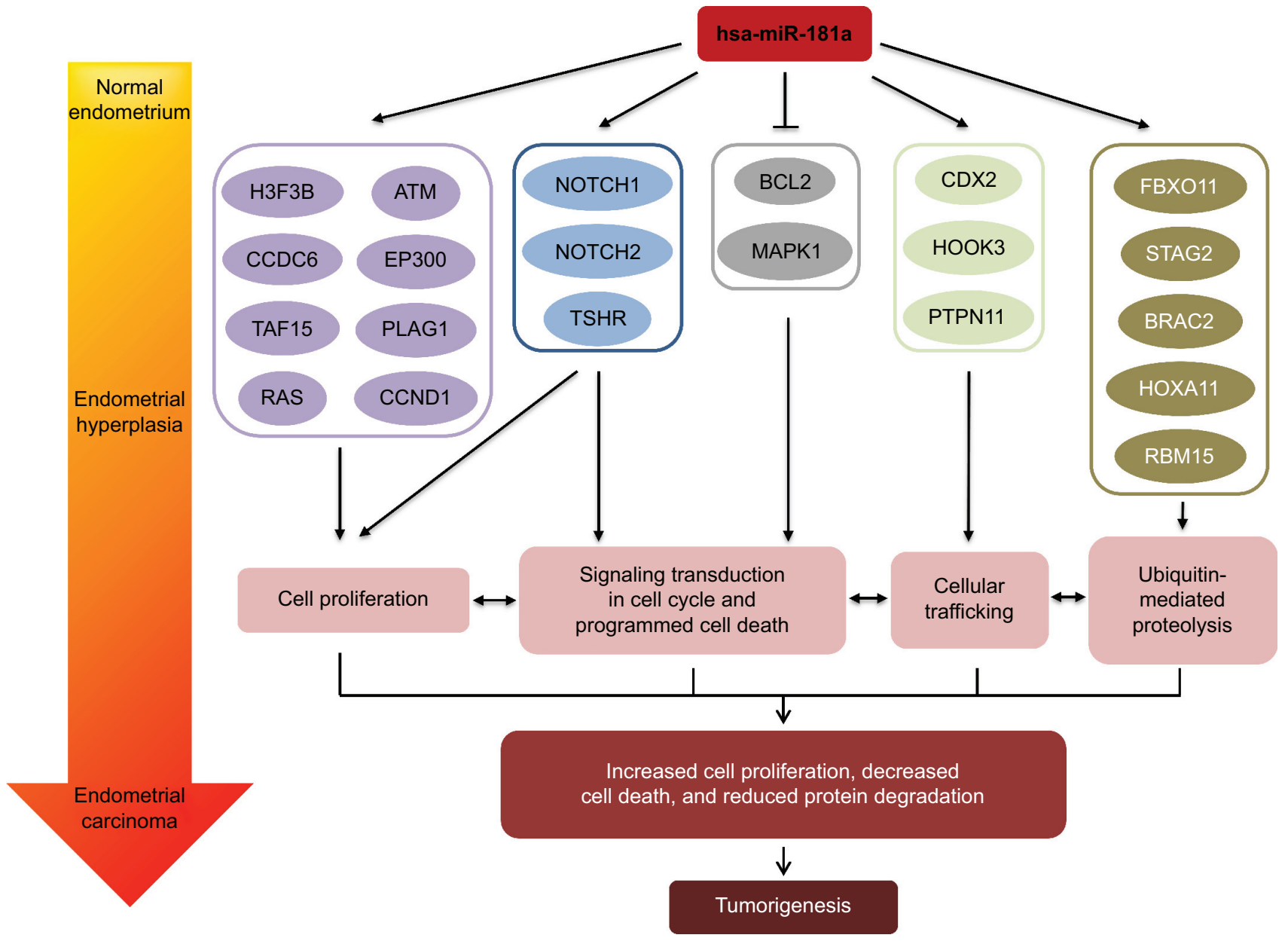

Figure 12 Proposed mechanisms for how hsa-miR-18Ia regulates cancer genes and may serve as an oncogene for the development of EC.

Notes: hsa-miR-I8Ia may play a role in the tumorigenesis of EC via multiple pathways that interplay with many important oncogenes and tumor suppressors. hsa-miR-I8Ia may interact with H3F3B, ATM, CCDC6, TAFI5, RAS, and PLAGI to promote cell proliferation. It may suppress cell apoptosis via interaction with NOTCHI, NOTCH2, MAPKI, and BCL2. It may affect ubiquitin-mediated proteolysis via regulation of FBXOII, STAG2, BRCA2, HOXAII, and RBMI5. All these actions may transform the normal endometrial cells into tumor cells.

Abbreviation: EC, endometrial cancer; ATM, ATM serine/threonine kinase; BCL2, B-cell lymphoma 2; CCDC6, coiled-coil domain containing 6; PLAGI, pleiomorphic adenoma gene I; MAPKI, mitogen activated kinase-like protein I; RAS, rat sarcoma viral oncogene homolog; BRCA2, breast cancer 2, early onset; STAG2, stromal antigen 2; HOXAII, homeobox AII; RBMI5, RNA binding motif protein I5; NOTCHI, notch I; H3F3B, H3 histone, family 3B; FBXOII, F-box protein II; TAFI5, TAFI5 RNA polymerase II, TATA box binding protein (TBP)-associated factor, $68 \mathrm{kDa}$; HOOK3, hook microtubule-tethering protein 3; PTPNII, protein tyrosine phosphatase, nonreceptor type II; TSHR, thyroid stimulating hormone receptor; CDX2, caudal type homeobox 2; EP300, EIA binding protein p300.

Table 17 Association between hsa-miR-I8Ia expression and pathological characteristics of EC

\begin{tabular}{|c|c|c|c|c|}
\hline $\begin{array}{l}\text { Pathological } \\
\text { characteristics }\end{array}$ & $\mathbf{N}$ & $\Delta \mathrm{Ct}$ & $F$ & $P$ \\
\hline \multicolumn{5}{|l|}{ FIGO stage } \\
\hline I+II & 38 & $-2.929 \pm 2.306$ & 7.120 & 0.011 \\
\hline $\mathrm{III+VI}$ & 9 & $-5.160 \pm 2.002$ & & \\
\hline \multicolumn{5}{|l|}{ Tumor grade } \\
\hline GI & 32 & $-3.152 \pm 2.528$ & 0.723 & 0.400 \\
\hline $\mathrm{G} 2+\mathrm{G} 3$ & 15 & $-3.792 \pm 2.118$ & & \\
\hline \multicolumn{5}{|l|}{ Lymph migration } \\
\hline No & 42 & $-3.183 \pm 2.370$ & 2.095 & 0.155 \\
\hline Yes & 5 & $-4.808 \pm 2.40 \mathrm{I}$ & & \\
\hline \multicolumn{5}{|c|}{ Myometrial invasion } \\
\hline$\leq \mathrm{I} / 2$ & 39 & $-3.086 \pm 2.406$ & 3.033 & 0.088 \\
\hline$>1 / 2$ & 8 & $-4.674 \pm 2.015$ & & \\
\hline
\end{tabular}

Abbreviations: $\Delta \mathrm{Ct}$, normalized threshold cycle; $\mathrm{EC}$, endometrial cancer; FIGO, International Federation of Gynecology and Obstetrics. and chronic leukemias. ${ }^{76-81}$ Ciafrè et al,${ }^{82}$ firstly reported that the expression of hsa-miR-181a was significantly downregulated in primary glioblastomas and human glioblastoma cell lines compared with normal brain tissue. As in glioblastoma, significant downregulation of hsa-miR-181a was also observed in squamous lung cell carcinoma, ${ }^{83}$ oral squamous cell carcinoma, ${ }^{71}$ luminal A-like breast cancer, ${ }^{67}$ and non-small-cell lung cancer ${ }^{84}$ However, hsa-miR-181a was significantly overexpressed in MCF-7 breast cancer cells ${ }^{85}$ colorectal cancer, ${ }^{28}$ and hepatocellular carcinoma cells. ${ }^{56,86,87}$ Hsa-miR-181a was upregulated in acute myeloid leukemia, ${ }^{76}$ especially in the M1 and M2 subtypes, and in myelodysplastic syndromes ${ }^{88}$ but downregulated in multiple myeloma ${ }^{89}$ and chronic lymphocyte leukemia. ${ }^{79,90}$ hsamiR-181a can serve as an oncogene $e^{46,54,56-58,60,61,65,66,68-70,87,91}$ 
or tumor suppressor, ${ }^{55,67,71,75,78,79,92}$ implicating its multifaceted and complex roles in the regulation of its target genes and signaling pathways associated with cancer initiation, growth, development, progression, and metastasis.

In the present study, our bioinformatic study predicted that hsa-miR-181a could regulate a large number of targets, including proteins that participate in regulation of cell proliferation, cell cycle, apoptosis, autophagy, metabolism, signaling transduction, and transport. A further search in TarBase and miRTarBase identified 313 targets of hsa-miR-181a-5p, and 22 of these genes are cancer genes that play critical roles in the tumorigenesis of various cancers.

During the prediction process, we employed ten different predicting programs that are based on different matching criteria and calculating algorithms. All the algorithms displayed disappointing predictive accuracy and ability when compared with the validated targets of hsa-miR-181a. It appears that there is a need to refine or combine these algorithms to improve their predictive accuracy and ability. Indeed, most of these prediction algorithms, including RNAhybrid, miRanda, TargetScan, DIANA microT, and PicTar exhaustively analyze all the possible miRNA: mRNA pairs, searching for structural evidence that could suggest the existence of an interaction. Although these approaches are significantly cheaper than those based on experimental validation, results of these methods are in many cases uncorrelated to each other, and their degree of overlap is low as shown in this study. The weakness of these algorithms depends on many factors, especially on the impossibility of incorporating in a single model all the possible interplaying variants/factors that can affect miRNA targeting and the prediction outcomes, especially in mammals. Different results can also depend on the approach used and on the rules considered for the miRNA targeting, as well as on the type of resource of sequences they use as a reference dataset. ${ }^{93}$ Shirdel et al $^{94}$ found that the precision and recall values computed against validated interactions of a specific algorithm were generally poor, but a combination of these algorithms can improve the prediction precision. Recently, some machine learning approaches have been incorporated, to learn to combine the outputs of distinct prediction algorithms and improve their accuracy. ${ }^{95-97}$ Zhang and Verbeek ${ }^{97}$ proposed the application of a supervised learning algorithm, ie, a Bayesian network learner, to distinct sets of features considered in three prediction algorithms, including RNAhybrid, miRanda, and TargetScan. Pio et a ${ }^{96}$ proposed a semisupervised ensemble learning approach using miRTarBase as the set of labeled (positive) interactions and microRNA Data Integration
Portal (mirDIP) as the set of unlabeled interactions, and the predictive accuracy was improved.

We next compared the expression levels of hsa-miR-181a in normal endometrium, endometrial hyperplasia, and type I and type II EC. We found that the expression level of hsa-miR-181a was significantly higher in EC than that in normal endometrium and that advanced EC exhibited a higher expression level of hsa-miR-181a. These observations demonstrate that there was an association between the expression level of hsa-miR-181a and the progression of EC and that hsa-miR-181a might serve as an oncogene in the development and progression of EC.

Many miRNAs are aberrantly expressed in cancer, resulting in functional alterations in cell differentiation, proliferation, migration, invasion, programmed cell death, and survival. 5,6,8,53 A number of oncogenes and tumor-suppressor genes could be potentially regulated by miRNAs. miRNAs are presumed to be a class of genes involved in human tumorigenesis, and miRNA-mediated gene regulation is an important cellular biologic process in cancer development. ${ }^{5,6,8,53}$ For example, let-7 acts as tumor suppressor gene, which was found to be downregulated in lung tumors and associated with a poor postoperative prognosis. ${ }^{98}$ It has been showed that the RAS oncogene was regulated by let- 7 and that a decreased expression level of let-7 in lung cancer resulted in an increase in the expression level of the RAS oncogene. ${ }^{99}$

Many studies have showed that upregulation of hsamiR-181a promotes carcinogenesis, cancer cell growth, and metastasis in a variety of cancers, via regulation of a number of molecular targets and signaling pathways related to cell proliferation, invasion, migration, survival, and cell death. ${ }^{46,54,56-58,60,61,65,66,68-70,87,91}$ Zou et al ${ }^{87}$ observed an increase in the expression level of hsa-miR-181a, which may contribute to the development and progression of hepatocellular carcinoma via targeting of E2F transcription factor 5, p130-binding (E2F5). hsa-miR-181a was also upregulated in hepatocellular cancer stem cells. ${ }^{86,100}$ Silencing hsa-miR-181 led to a decreased motility and invasion of hepatocellular cancer stem cells, via targeting of the putative tumor suppressor Ras association domain family 1 isoform A (RASSF1), metalloproteinase inhibitor 3 (ie, TIMP3), and nemo-like kinase (NLK). ${ }^{100}$ hsamiR-181 could directly target hepatic transcriptional regulators of differentiation, including caudal type homeobox 2 (CDX2), GATA binding protein 6 (GATA6), and NLK, an inhibitor of Wnt/ $\beta$-catenin signaling. ${ }^{86}$ hsa-miR-181a promoted tumor growth and liver metastasis in colorectal cancer patients by targeting the tumor suppressor WNT inhibitory factor 1 (WIF1). ${ }^{28}$ hsa-miR-181a was most elevated in these colorectal cancer patients with liver metastases and could serve as an independent prognostic factor of poor overall survival. ${ }^{28}$ hsa-miR-181a 
showed a potent tumor-promoting effect through inhibition of the expression of WIF1 and promotion of epithelial-mesenchymal transition. ${ }^{28}$ Moreover, upregulation of hsa-miR-181a plays a potential role in the development of gastric cancer by targeting the tumor suppressor ATM serine/threonine kinase (ATM). Consequently, it leads to promotion of gastric cancer cell proliferation and inhibition of apoptosis. Wei et $\mathrm{al}^{101}$ showed that the PTEN/Akt signaling pathway was involved in the regulatory effect of hsa-miR-181a in the development of colon cancer, by promoting cell growth. hsa-miR-181a also played an important role in ovarian cancer progression, by promoting epithelial-mesenchymal transition. ${ }^{54}$ These data indicate that hsa-miR-181a may function as oncogenic miRNA in cancer development and progression. In agreement with previous studies, our findings showed that there was a significant increase in the expression level of hsa-miR-181a in EC compared with that in normal endometrium. Moreover, our results showed that advanced EC had a significant higher expression level of hsa-miR-181a than that in early stage of EC, suggesting that hsa-miR-181a may have a critical role in tumor metastasis of advanced EC.

On the other hand, hsa-miR-181 may function as a tumor suppressor. In glioma, hsa-miR-181a was shown to be downregulated. ${ }^{30}$ Both hsa-miR-181a and hsa-miR-181b triggered growth inhibition, induced apoptosis, and inhibited invasion in glioma cells. Transiently overexpressed hsa-miR-181a significantly sensitized malignant glioma U87MG cells to radiation with downregulated BCL2. ${ }^{55}$ In chronic lymphocytic leukemia, hsa-miR-181a together with hsa-miR-15a, hsa-miR-16-1, hsa-miR-29b, and hsa-miR-181b were all downregulated. ${ }^{90}$ These miRNAs may play a role in the pathogenesis of chronic lymphocytic leukemia and serve as new biomarkers for the prediction of prognosis in chronic lymphocytic leukemia. hsa-miR-181a expression level was found to be significantly lower in poor prognosis patients, and a low expression of hsa-miR-181a and hsa-miR-181b was associated with shorter overall survival and treatment-free survival in patients with chronic lymphocytic leukemia. ${ }^{79}$ Furthermore, hsa-miR-181a inhibited the migration, invasion, and proliferation of salivary adenoid cystic carcinoma cells, and suppressed tumor growth and lung metastasis in nude mice, via targeting of MAP2K1, MAPK1, and SNAI2. ${ }^{75}$

Based on our DAVID and KEGG pathway analysis, PI3K/Akt, MAPK, and Wnt signaling pathways played important roles in the development of type I EC. CCND1, HRAS, and KRAS are all key components in these pathways, which are all validated targets of hsa-miR-181a.

The expression level of hsa-miR-181a has been proposed as a potential biomarker for assessing prognosis and therapeutic response in cancer. Ouyang et al ${ }^{102}$ suggested that hsa-miR-181a may be a potential biomarker for predicting chemoresistance in the treatment of triple negative breast cancer. It also has been showed that hsa-miRNA-181a enhanced the chemoresistance of human cervical squamous cell carcinoma to cisplatin by targeting protein kinase $C \delta^{69}$ and that hsa-miR-181a may serve an oncologic miRNA biomarker for luminal A-like breast cancer. ${ }^{67}$ Interestingly, Pichler et al ${ }^{59}$ showed a reverse correlation between hsa-miR-181a expression level and survival rate in patients with colorectal cancer. In our study, we observed a significant difference in the expression level of hsa-miR-181a among normal endometrium, endometrial hyperplasia, and $\mathrm{EC}$, and a higher expression level of hsa-miR-181a in advanced EC. Our findings suggest that the expression level of hsa-miR-181a might serve as a useful biomarker for the prediction of prognosis of EC in clinic.

In summary, our bioinformatics studies have showed that hsa-miR-181a might regulate a large number of target genes that are important in the regulation of critical cell processes. To date, 313 targets of hsa-miR-181a have been validated, and 22 of these targets are cancer genes. Many of these genes are involved in tumorigenesis of various cancers, including EC. Our data demonstrate that hsa-miR-181a is upregulated in $\mathrm{EC}$, with a possible role in the development and progression of EC (Figure 12). It might serve as a new biomarker for prognosis prediction in EC in clinical practice and has important implication in the treatment of EC. More mechanistic and functional studies are needed to validate the role of hsa-miR-181a in the pathogenesis of EC and to establish the association between the expression level of hsa-miR-181a and the clinical phenotypes, including disease status and therapeutic response of EC to chemo- and radiotherapy.

\section{Acknowledgment}

This work was supported by Guangdong Natural Science Foundation (grant number S2012010010024), Guangzhou, People's Republic of China. Dr Zhi-Wei Zhou is a postdoctoral fellow supported by the College of Pharmacy, University of South Florida, Tampa, FL, USA.

\section{Disclosure}

The authors report no conflicts of interest in this work.

\section{References}

1. Croce CM, Calin GA. miRNAs, cancer, and stem cell division. Cell. 2005; 122(1):6-7.

2. Yates LA, Norbury CJ, Gilbert RJ. The long and short of microRNA. Cell. 2013;153(3):516-519.

3. Lu J, Getz G, Miska EA, et al. MicroRNA expression profiles classify human cancers. Nature. 2005;435(7043):834-838. 
4. Banno K, Yanokura M, Kisu I, Yamagami W, Susumu N, Aoki D. MicroRNAs in endometrial cancer. Int J Clin Oncol. 2013;18(2):186-192.

5. Lujambio A, Lowe SW. The microcosmos of cancer. Nature. 2012; 482(7385):347-355.

6. Esquela-Kerscher A, Slack FJ. Oncomirs - microRNAs with a role in cancer. Nat Rev Cancer. 2006;6(4):259-269.

7. Ryan BM, Robles AI, Harris CC. Genetic variation in microRNA networks: the implications for cancer research. Nat Rev Cancer. 2010; 10(6):389-402.

8. Nana-Sinkam SP, Croce CM. Clinical applications for microRNAs in cancer. Clin Pharmacol Ther. 2013;93(1):98-104.

9. Ritchie W, Rasko JE. Refining microRNA target predictions: sorting the wheat from the chaff. Biochem Biophys Res Commun. 2014; 445(4):780-784.

10. Peterson SM, Thompson JA, Ufkin ML, Sathyanarayana P, Liaw L, Congdon CB. Common features of microRNA target prediction tools. Front Genet. 2014;5:23.

11. Ferlay J, Soerjomataram I, Ervik M, et al. GLOBOCAN 2012 v1.0, Cancer Incidence and Mortality Worldwide: IARC CancerBase No. 11 [Internet]. Lyon: International Agency for Research on Cancer; 2013. Available from: http://globocan.iarc.fr. Accessed November 10, 2014.

12. Howlader N, Noone AM, Krapcho M, et al. SEER Cancer Statistics Review, 1975-2011. Bethesda, MD: National Cancer Institute; 2014.

13. Balch C, Matei DE, Huang TH, Nephew KP. Role of epigenomics in ovarian and endometrial cancers. Epigenomics. 2010;2(3): 419-447.

14. Amant F, Moerman P, Neven P, Timmerman D, Van Limbergen E, Vergote I. Endometrial cancer. Lancet. 2005;366(9484):491-505.

15. Colombo N, Preti E, Landoni F, et al; ESMO Guidelines Working Group. Endometrial cancer: ESMO Clinical Practice Guidelines for diagnosis, treatment and follow-up. Ann Oncol. 2013;24 Suppl 6: vi33-vi38.

16. Hecht JL, Mutter GL. Molecular and pathologic aspects of endometrial carcinogenesis. J Clin Oncol. 2006;24(29):4783-4791.

17. Yeramian A, Moreno-Bueno G, Dolcet X, et al. Endometrial carcinoma: molecular alterations involved in tumor development and progression. Oncogene. 2013;32(4):403-413.

18. National Cancer Institute. Endometrial Cancer. 2014. Available from http://www.cancer.gov/cancertopics/types/endometrial. Accessed December 15, 2014.

19. Dong P, Kaneuchi M, Konno Y, Watari H, Sudo S, Sakuragi N. Emerging therapeutic biomarkers in endometrial cancer. Biomed Res Int. 2013; 2013:130362.

20. Ow TJ, Sandulache VC, Skinner HD, Myers JN. Integration of cancer genomics with treatment selection: from the genome to predictive biomarkers. Cancer. 2013;119(22):3914-3928.

21. Mitra S, Das S, Chakrabarti J. Systems biology of cancer biomarker detection. Cancer Biomark. 2013;13(4):201-213.

22. Widschwendter M, Jones A, Teschendorff AE. Epigenetics makes its mark on women-specific cancers - an opportunity to redefine oncological approaches? Gynecol Oncol. 2013;128(1):134-143.

23. Schwarzenbach H, Hoon DS, Pantel K. Cell-free nucleic acids as biomarkers in cancer patients. Nat Rev Cancer. 2011;11(6):426-437.

24. Kasinski AL, Slack FJ. Epigenetics and genetics. MicroRNAs en route to the clinic: progress in validating and targeting microRNAs for cancer therapy. Nat Rev Cancer. 2011;11(12):849-864.

25. Chen CZ, Li L, Lodish HF, Bartel DP. MicroRNAs modulate hematopoietic lineage differentiation. Science. 2004;303(5654):83-86.

26. Lin Y, Nie Y, Zhao J, et al. Genetic polymorphism at miR-181a binding site contributes to gastric cancer susceptibility. Carcinogenesis. 2012; 33(12):2377-2383.

27. Havelange V, Stauffer N, Heaphy CC, et al. Functional implications of microRNAs in acute myeloid leukemia by integrating microRNA and messenger RNA expression profiling. Cancer. 2011;117(20):4696-4706.

28. Ji D, Chen Z, Li M, et al. MicroRNA-181a promotes tumor growth and liver metastasis in colorectal cancer by targeting the tumor suppressor WIF-1. Mol Cancer. 2014;13:86.
29. Fei J, Li Y, Zhu X, Luo X. miR-181 a post-transcriptionally downregulates oncogenic RalA and contributes to growth inhibition and apoptosis in chronic myelogenous leukemia (CML). PLoS One. 2012;7(3):e32834.

30. Shi L, Cheng Z, Zhang J, et al. hsa-mir-181a and hsa-mir-181b function as tumor suppressors in human glioma cells. Brain Res. 2008;1236: 185-193.

31. Panda H, Chuang TD, Luo X, Chegini N. Endometrial miR-181a and miR-98 expression is altered during transition from normal into cancerous state and target PGR, PGRMC1, CYP19A1, DDX3X, and TIMP3. $J$ Clin Endocrinol Metab. 2012;97(7):E1316-E1326.

32. Kozomara A, Griffiths-Jones S. miRBase: annotating high confidence microRNAs using deep sequencing data. Nucleic Acids Res. 2014;42(Database issue):D68-D73.

33. Kozomara A, Griffiths-Jones S. miRBase: integrating microRNA annotation and deep-sequencing data. Nucleic Acids Res. 2011;39(Database issue):D152-D157.

34. Griffiths-Jones S, Grocock RJ, van Dongen S, Bateman A, Enright AJ. miRBase: microRNA sequences, targets and gene nomenclature. Nucleic Acids Res. 2006;34(Database issue):D140-D144.

35. Betel D, Koppal A, Agius P, Sander C, Leslie C. Comprehensive modeling of microRNA targets predicts functional non-conserved and non-canonical sites. Genome Biol. 2010;11(8):R90.

36. Maragkakis M, Alexiou P, Papadopoulos GL, et al. Accurate microRNA target prediction correlates with protein repression levels. BMC Bioinformatics. 2009;10:295.

37. Paraskevopoulou MD, Georgakilas G, Kostoulas N, et al. DIANA-microT web server v5.0: service integration into miRNA functional analysis workflows. Nucleic Acids Res. 2013;41(Web Server issue):W169-W173.

38. Wang X. miRDB: a microRNA target prediction and functional annotation database with a wiki interface. RNA. 2008;14(6):1012-1017.

39. Miranda KC, Huynh T, Tay Y, et al. A pattern-based method for the identification of MicroRNA binding sites and their corresponding heteroduplexes. Cell. 2006;126(6):1203-1217.

40. Bandyopadhyay S, Mitra R. TargetMiner: microRNA target prediction with systematic identification of tissue-specific negative examples. Bioinformatics. 2009;25(20):2625-2631.

41. Lewis BP, Burge CB, Bartel DP. Conserved seed pairing, often flanked by adenosines, indicates that thousands of human genes are microRNA targets. Cell. 2005;120(1):15-20.

42. Garcia DM, Baek D, Shin C, Bell GW, Grimson A, Bartel DP. Weak seed-pairing stability and high target-site abundance decrease the proficiency of 1sy-6 and other microRNAs. Nat Struct Mol Biol. 2011;18(10): $1139-1146$

43. Krek A, Grün D, Poy MN, et al. Combinatorial microRNA target predictions. Nat Genet. 2005;37(5):495-500.

44. Dweep H, Sticht C, Pandey P, Gretz N. miRWALK - database: prediction of possible miRNA binding sites by "walking" the genes of three genomes. J Biomed Inform. 2011;44(5):839-847.

45. Vergoulis T, Vlachos IS, Alexiou P, et al. TarBase 6.0: capturing the exponential growth of miRNA targets with experimental support. Nucleic Acids Res. 2012;40(Database issue):D222-D229.

46. Hsu SD, Tseng YT, Shrestha S, et al. miRTarBase update 2014: an information resource for experimentally validated miRNA-target interactions. Nucleic Acids Res. 2014;42(Database issue):D78-D85.

47. Futreal PA, Coin L, Marshall M, et al. A census of human cancer genes. Nat Rev Cancer. 2004;4(3):177-183.

48. Vogelstein B, Papadopoulos N, Velculescu VE, Zhou S, Diaz LA Jr, Kinzler KW. Cancer genome landscapes. Science. 2013;339(6127): $1546-1558$.

49. Huang da W, Sherman BT, Lempicki RA. Systematic and integrative analysis of large gene lists using DAVID bioinformatics resources. Nat Protoc. 2009;4(1):44-57.

50. Huang da W, Sherman BT, Tan Q, et al. DAVID bioinformatics resources: expanded annotation database and novel algorithms to better extract biology from large gene lists. Nucleic Acids Res. 2007;35(Web Server issue):W169-W175. 
51. Pecorelli S. Revised FIGO staging for carcinoma of the vulva, cervix, and endometrium. Int J Gynaecol Obstet. 2009;105(2):103-104.

52. Di Leva G, Garofalo M, Croce CM. MicroRNAs in cancer. Annu Rev Pathol. 2014;9:287-314.

53. Gilabert-Estelles J, Braza-Boils A, Ramon LA, et al. Role of microRNAs in gynecological pathology. Curr Med Chem. 2012;19(15): 2406-2413.

54. Parikh A, Lee C, Peronne J, et al. microRNA-181a has a critical role in ovarian cancer progression through the regulation of the epithelialmesenchymal transition. Nat Commun. 2014;5:2977.

55. Chen G, Zhu W, Shi D, et al. MicroRNA-181a sensitizes human malignant glioma U87MG cells to radiation by targeting Bcl-2. Oncol Rep. 2010;23(4): 997-1003

56. Brockhausen J, Tay SS, Grzelak CA, et al. miR-181a mediates TGF$\beta$-induced hepatocyte EMT and is dysregulated in cirrhosis and hepatocellular cancer. Liver Int. 2015;35(1):240-253.

57. Nishimura J, Handa R, Yamamoto H, et al. microRNA-181a is associated with poor prognosis of colorectal cancer. Oncol Rep. 2012; 28(6):2221-2226.

58. Zou C, Chen J, Chen K, et al. Functional analysis of miR-181a and Fas involved in hepatitis B virus-related hepatocellular carcinoma pathogenesis. Exp Cell Res. 2015 [Epub ahead of print].

59. Pichler M, Winter E, Ress AL, et al. miR-181a is associated with poor clinical outcome in patients with colorectal cancer treated with EGFR inhibitor. J Clin Pathol. 2014;67(3):198-203.

60. Zhang X, Nie Y, Du Y, Cao J, Shen B, Li Y. MicroRNA-181a promotes gastric cancer by negatively regulating tumor suppressor KLF6. Tumour Biol. 2012;33(5):1589-1597.

61. Zhang X, Nie Y, Li X et al., MicroRNA-181a functions as an oncomir in gastric cancer by targeting the tumour suppressor gene ATM. Pathol Oncol Res. 2014;20(2):381-389.

62. Galluzzi L, Morselli E, Vitale I, et al. miR-181a and miR-630 regulate cisplatin-induced cancer cell death. Cancer Res. 2010;70(5): 1793-1803.

63. Farazi TA, Horlings HM, Ten Hoeve JJ, et al. MicroRNA sequence and expression analysis in breast tumors by deep sequencing. Cancer Res. 2011;71(13):4443-4453.

64. Guo LJ, Zhang QY. Decreased serum miR-181a is a potential new tool for breast cancer screening. Int J Mol Med. 2012;30(3):680-686.

65. Taylor MA, Sossey-Alaoui K, Thompson CL, Danielpour D, Schiemann WP. TGF- $\beta$ upregulates miR-181a expression to promote breast cancer metastasis. J Clin Invest. 2013;123(1):150-163.

66. Bisso A, Faleschini M, Zampa F, et al. Oncogenic miR-181a/b affect the DNA damage response in aggressive breast cancer. Cell Cycle. 2013 12(11):1679-1687.

67. McDermott AM, Miller N, Wall D, et al. Identification and validation of oncologic miRNA biomarkers for luminal A-like breast cancer. PLoS One. 2014;9(1):e87032.

68. Ke G, Liang L, Yang JM, et al. MiR-181a confers resistance of cervical cancer to radiation therapy through targeting the pro-apoptotic PRKCD gene. Oncogene. 2013;32(25):3019-3027.

69. Chen Y, Ke G, Han D, Liang S, Yang G, Wu X. MicroRNA-181a enhances the chemoresistance of human cervical squamous cell carcinoma to cisplatin by targeting PRKCD. Exp Cell Res. 2014;320(1):12-20.

70. Jianwei Z, Fan L, Xiancheng L, Enzhong B, Shuai L, Can L. MicroRNA $181 \mathrm{a}$ improves proliferation and invasion, suppresses apoptosis of osteosarcoma cell. Tumour Biol. 2013;34(6):3331-3337.

71. Shin KH, Bae SD, Hong HS, Kim RH, Kang MK, Park NH. miR-181a shows tumor suppressive effect against oral squamous cell carcinoma cells by downregulating K-ras. Biochem Biophys Res Commun. 2011;404(4):896-902.

72. Liu M, Wang J, Huang H, Hou J, Zhang B, Wang A. miR-181a-Twist1 pathway in the chemoresistance of tongue squamous cell carcinoma. Biochem Biophys Res Commun. 2013;441(2):364-370.

73. Alencar AJ, Malumbres R, Kozloski GA, et al. MicroRNAs are independent predictors of outcome in diffuse large B-cell lymphoma patients treated with R-CHOP. Clin Cancer Res. 2011;17(12):4125-4135.
74. Keutgen XM, Filicori F, Crowley MJ, et al. A panel of four miRNAs accurately differentiates malignant from benign indeterminate thyroid lesions on fine needle aspiration. Clin Cancer Res. 2012;18(7): 2032-2038.

75. He Q, Zhou X, Li S, et al. MicroRNA-181a suppresses salivary adenoid cystic carcinoma metastasis by targeting MAPK-Snai2 pathway. Biochim Biophys Acta. 2013;1830(11):5258-5266.

76. Debernardi S, Skoulakis S, Molloy G, Chaplin T, Dixon-McIver A, Young BD. MicroRNA miR-181a correlates with morphological subclass of acute myeloid leukaemia and the expression of its target genes in global genome-wide analysis. Leukemia. 2007;21(5):912-916.

77. Cuesta R, Martínez-Sánchez A, Gebauer F. miR-181a regulates capdependent translation of p27(kip1) mRNA in myeloid cells. Mol Cell Biol. 2009;29(10):2841-2851.

78. Bai H, Cao Z, Deng C, Zhou L, Wang C. miR-181a sensitizes resistant leukaemia HL-60/Ara-C cells to Ara-C by inducing apoptosis. J Cancer Res Clin Oncol. 2012;138(4):595-602.

79. Zhu DX, Zhu W, Fang C, et al. miR-181a/b significantly enhances drug sensitivity in chronic lymphocytic leukemia cells via targeting multiple anti-apoptosis genes. Carcinogenesis. 2012;33(7):1294-1301.

80. Dahlhaus M, Schult C, Lange S, Freund M, Junghanss C. MicroRNA 181a influences the expression of $H M G B 1$ and $C D 4$ in acute leukemias. Anticancer Res. 2013;33(2):445-452.

81. Lin S, Pan L, Guo S, et al. Prognostic role of microRNA-181a/b in hematological malignancies: a meta-analysis. PLoS One. 2013;8(3): e59532.

82. Ciafrè SA, Galardi S, Mangiola A, et al. Extensive modulation of a set of microRNAs in primary glioblastoma. Biochem Biophys Res Commun. 2005;334(4):1351-1358.

83. Gao W, Shen H, Liu L, Xu J, Xu J, Shu Y. MiR-21 overexpression in human primary squamous cell lung carcinoma is associated with poor patient prognosis. J Cancer Res Clin Oncol. 2011;137(4):557-566.

84. Gao W, Yu Y, Cao H, et al. Deregulated expression of miR-21, miR143 and miR-181a in non small cell lung cancer is related to clinicopathologic characteristics or patient prognosis. Biomed Pharmacother. 2010;64(6):399-408.

85. Miller TE, Ghoshal K, Ramaswamy B, et al. MicroRNA-221/222 confers tamoxifen resistance in breast cancer by targeting p27Kip1. J Biol Chem. 2008;283(44):29897-29903.

86. Ji J, Yamashita T, Budhu A, et al. Identification of microRNA-181 by genome-wide screening as a critical player in EpCAM-positive hepatic cancer stem cells. Hepatology. 2009;50(2):472-480.

87. Zou C, Li Y, Cao Y, et al. Up-regulated MicroRNA-181a induces carcinogenesis in hepatitis B virus-related hepatocellular carcinoma by targeting E2F5. BMC Cancer. 2014;14:97.

88. Pons A, Nomdedeu B, Navarro A, et al. Hematopoiesis-related microRNA expression in myelodysplastic syndromes. Leuk Lymphoma. 2009;50(11):1854-1859.

89. Pichiorri F, Suh SS, Ladetto M, et al. MicroRNAs regulate critical genes associated with multiple myeloma pathogenesis. Proc Natl Acad Sci U S A. 2008;105(35):12885-12890.

90. Zhu DX, Miao KR, Fang C, et al. Aberrant microRNA expression in Chinese patients with chronic lymphocytic leukemia. Leuk Res. 2011; 35(6):730-734.

91. Albulescu R. TGF- $\beta$ upregulates miR-181a expression to promote breast cancer metastasis. Biomark Med. 2013;7(2):204.

92. Jiao X, Zhao L, Ma M, et al. MiR-181a enhances drug sensitivity in mitoxantone-resistant breast cancer cells by targeting breast cancer resistance protein (BCRP/ABCG2). Breast Cancer Res Treat. 2013; 139(3):717-730.

93. Ritchie W, Flamant S, Rasko JE. Predicting microRNA targets and functions: traps for the unwary. Nat Methods. 2009;6(6):397-398.

94. Shirdel EA, Xie W, Mak TW, Jurisica I. NAViGaTing the micronomeusing multiple microRNA prediction databases to identify signalling pathway-associated microRNAs. PLoS One. 2011;6(2):e17429.

95. Godsey B. Discovery of miR-mRNA interactions via simultaneous Bayesian inference of gene networks and clusters using sequence-based predictions and expression data. J Integr Bioinform. 2013;10(1):227. 
96. Pio G, Malerba D, D'Elia D, Ceci M. Integrating microRNA target predictions for the discovery of gene regulatory networks: a semisupervised ensemble learning approach. BMC Bioinformatics. 2014;15 Suppl 1:S4.

97. Zhang Y, Verbeek FJ. Comparison and integration of target prediction algorithms for microRNA studies. J Integr Bioinform. 2010;7(3).

98. Takamizawa J, Konishi H, Yanagisawa K, et al. Reduced expression of the let-7 microRNAs in human lung cancers in association with shortened postoperative survival. Cancer Res. 2004;64(11):3753-3756.

99. Johnson SM, Grosshans H, Shingara J, et al. RAS is regulated by the let-7 microRNA family. Cell. 2005;120(5):635-647.

100. Meng F, Glaser SS, Francis H, et al. Functional analysis of microRNAs in human hepatocellular cancer stem cells. J Cell Mol Med. 2012; 16(1):160-173.

101. Wei Z, Cui L, Mei Z, Liu M, Zhang D. miR-181a mediates metabolic shift in colon cancer cells via the PTEN/AKT pathway. FEBS Lett. 2014; 588(9):1773-1779.

102. Ouyang M, Li Y, Ye S, et al. MicroRNA profiling implies new markers of chemoresistance of triple-negative breast cancer. PLoS One. 2014; 9(5):e96228.

103. Clevers H, Nusse R. Wnt/ $\beta$-catenin signaling and disease. Cell. 2012; 149(6):1192-1205.

104. MacDonald BT, Tamai K, He X. Wnt/ $\beta$-catenin signaling: components, mechanisms, and diseases. Dev Cell. 2009;17(1):9-26.

105. Pijnenborg JM, Kisters N, van Engeland M, et al. APC, $\beta$-catenin, and E-cadherin and the development of recurrent endometrial carcinoma. Int J Gynecol Cancer. 2004;14(5):947-956.

106. van der Zee M, Jia Y, Wang Y, et al. Alterations in Wnt- $\beta$-catenin and Pten signalling play distinct roles in endometrial cancer initiation and progression. J Pathol. 2013;230(1):48-58.

107. Liu Y, Meng F, Xu Y, et al. Overexpression of Wnt7a is associated with tumor progression and unfavorable prognosis in endometrial cancer. Int J Gynecol Cancer. 2013;23(2):304-311.

108. Zhao Y, Yang Y, Trovik J, et al. A novel wnt regulatory axis in endometrioid endometrial cancer. Cancer Res. 2014;74(18):5103-5117.

109. Liu Y, Patel L, Mills GB, et al. Clinical significance of CTNNB1 mutation and Wnt pathway activation in endometrioid endometrial carcinoma. J Natl Cancer Inst. 2014;106(9):dju245.

110. Malumbres M, Barbacid M. Cell cycle, CDKs and cancer: a changing paradigm. Nat Rev Cancer. 2009;9(3):153-166.
111. Plotnikov A, Zehorai E, Procaccia S, Seger R. The MAPK cascades: signaling components, nuclear roles and mechanisms of nuclear translocation. Biochim Biophys Acta. 2011;1813(9):1619-1633.

112. Ranganathan P, Weaver KL, Capobianco AJ. Notch signalling in solid tumours: a little bit of everything but not all the time. Nat Rev Cancer. 2011;11(5):338-351.

113. Suzuki T, Aoki D, Susumu N, Udagawa Y, Nozawa S. Imbalanced expression of TAN-1 and human Notch4 in endometrial cancers. Int J Oncol. 2000;17(6):1131-1139.

114. Cobellis L, Caprio F, Trabucco E, et al. The pattern of expression of Notch protein members in normal and pathological endometrium. J Anat. 2008;213(4):464-472.

115. Mitsuhashi Y, Horiuchi A, Miyamoto T, Kashima H, Suzuki A, Shiozawa T. Prognostic significance of Notch signalling molecules and their involvement in the invasiveness of endometrial carcinoma cells. Histopathology. 2012;60(5):826-837.

116. Jonusiene V, Sasnauskiene A, Lachej N, et al. Down-regulated expression of Notch signaling molecules in human endometrial cancer. Med Oncol. 2013;30(1):438.

117. Nickkho-Amiry M, McVey R, Holland C. Peroxisome proliferatoractivated receptors modulate proliferation and angiogenesis in human endometrial carcinoma. Mol Cancer Res. 2012;10(3):441-453.

118. Pengchong H, Tao H. Expression of IGF-1R, VEGF-C and D2-40 and their correlation with lymph node metastasis in endometrial adenocarcinoma. Eur J Gynaecol Oncol. 2011;32(6):660-664.

119. Dobrzycka B, Mackowiak-Matejczyk B, Kinalski M, Terlikowski SJ. Pretreatment serum levels of bFGF and VEGF and its clinical significance in endometrial carcinoma. Gynecol Oncol. 2013;128(3):454-460.

120. Saarelainen SK, Staff S, Peltonen N, et al. Endoglin, VEGF, and its receptors in predicting metastases in endometrial carcinoma. Tumour Biol. 2014;35(5):4651-4657.

121. Wang J, Taylor A, Showeil R, et al. Expression profiling and significance of VEGF-A, VEGFR2, VEGFR3 and related proteins in endometrial carcinoma. Cytokine. 2014;68(2):94-100.

122. Saharinen P, Eklund L, Pulkki K, Bono P, Alitalo K. VEGF and angiopoietin signaling in tumor angiogenesis and metastasis. Trends Mol Med. 2011;17(7):347-362. 


\section{Supplementary materials}

Table SI A full list of cancer genes, based on Futreal et al'. Reprinted by permission from Macmillan Publishers Ltd: Nature Reviews Cancer. Futreal PA, Coin L, Marshall M, et al. A census of human cancer genes. Nat Rev Cancer. 2004;4(3): I77-183. Copyright (C) 2004.'

Table S2 Predicted targets of hsa-miR- I I la-5p, by DIANA microT v5.0

Table S3 Predicted targets of hsa-miR-18Ia-5p, by miRanda-mirSVR

Table S4 Predicted targets of hsa-miR-18Ia-5p, by miRandamirSVR, that are cancer genes

Table S5 Predicted targets of hsa-miR-18Ia-5p, by miRDB

Table S6 Predicted targets of hsa-miR-18Ia-5p, by RNA22 v2

Table S7 Predicted targets of hsa-miR-181a-5p, by RNA22 v2, that are cancer genes

Table S8 Predicted targets of hsa-miR-I8Ia-5p, by TargetMiner Table S9 Predicted targets of hsa-miR-I8Ia-5p, by TargetScan 6.2

Table SIO Predicted targets of hsa-miR-18Ia-5p, by PicTar

Table SII Predicted targets of hsa-miR- |8|a-5p, by MicroCosm Targets v5

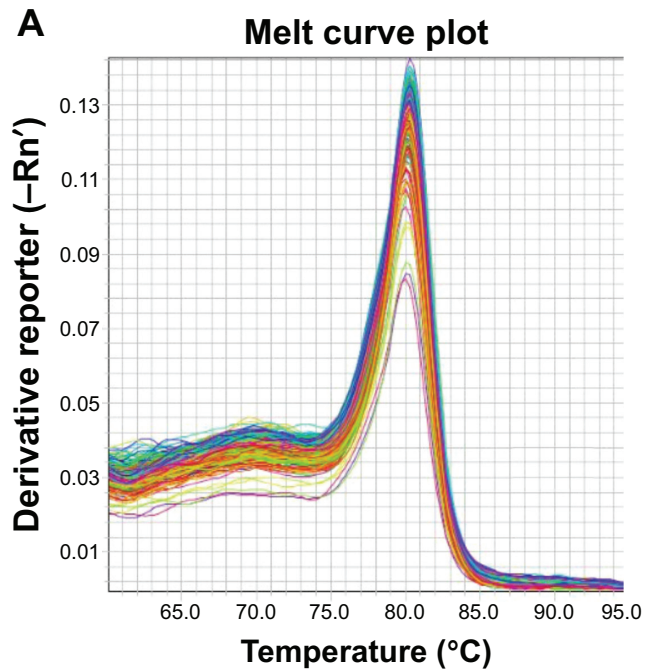

Table SI2 Predicted targets of hsa-miR-I8Ia-3p, by DIANA microT $v 5.0$

Table SI 3 Predicted targets of hsa-miR-18Ia-3p, by miRandamirSVR

Table SI4 Predicted targets of hsa-miR-I8Ia-3p, by miRandamirSVR, that are cancer genes

Table SI 5 Predicted targets of hsa-miR-I8Ia-3p, by miRDB

Table S16 Predicted targets of hsa-miR-181a-3p, by RNA22 v2

Table SI 7 Predicted targets of hsa-miR-18Ia-3p, by TargetMiner

Table SI 8 Predicted targets of hsa-miR-I8Ia-5p, by MicroCosm Targets v5

Table SI9 Predicted targets of hsa-miR-I8Ia, by miRWALK

Table S20 Predicted genes of hsa-miR-I8Ia, by miRWALK

Table S2I A summarized table that includes all the targets predicted to be regulated by hsa-miR-|8Ia by the eight algorithms
B Amplification plot

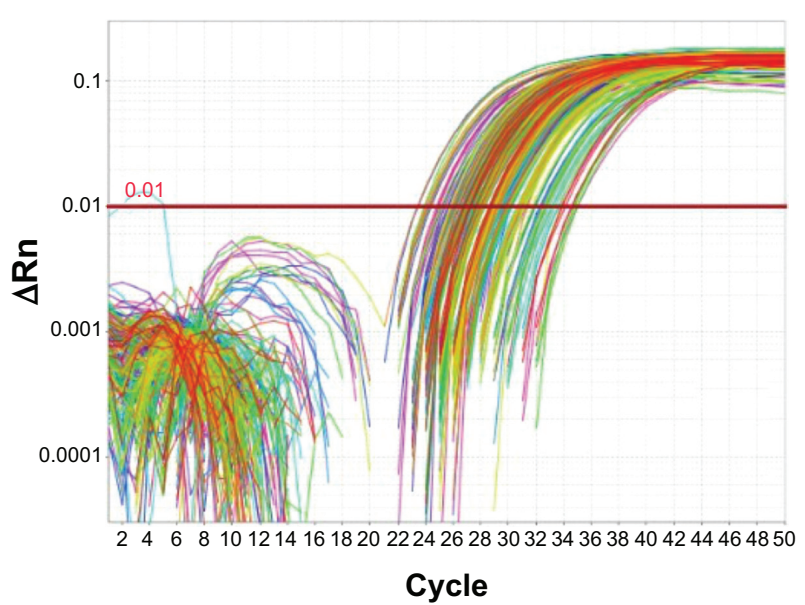

Figure SI Analysis of hsa-miR-I8I a expression in a human endometrial specimen by real-time PCR.

Notes: (A) Melting curve showing the single melt peak for hsa-miR-I8Ia and U6, respectively; and (B) the amplification plot of the target gene.

Abbreviation: $\mathrm{PCR}$, polymerase chain reaction.

\section{Reference}

1. Futreal PA, Coin L, Marshall M, et al. A census of human cancer genes. Nat Rev Cancer. 2004;4(3):177-183.

\section{Publish your work in this journal}

Drug Design, Development and Therapy is an international, peerreviewed open-access journal that spans the spectrum of drug design and development through to clinical applications. Clinical outcomes, patient safety, and programs for the development and effective, safe, and sustained use of medicines are a feature of the journal, which has also been accepted for indexing on PubMed Central. The manuscript management system is completely online and includes a very quick and fair peer-review system, which is all easy to use. Visit http://www.dovepress.com/testimonials.php to read real quotes from published authors.

Submit your manuscript here: http://www.dovepress.com/drug-design-development-and-therapy-journal 